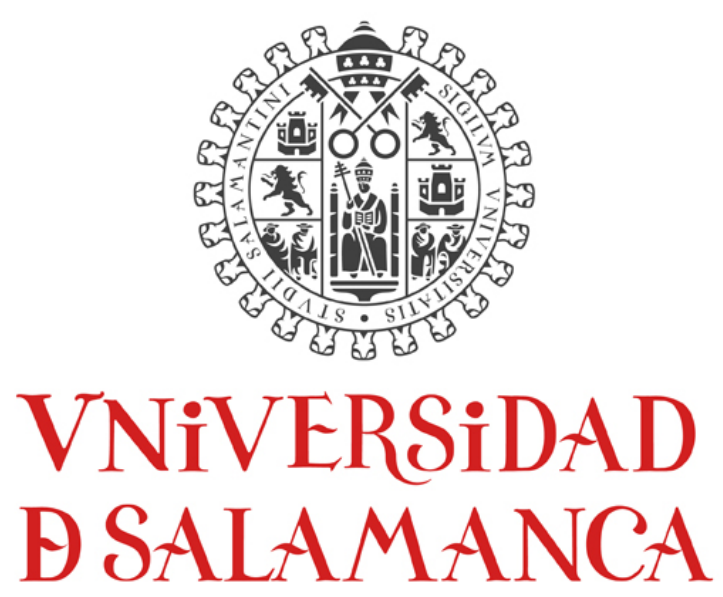

CAMPUS OF INTERNATIONAL EXCELLENCE

\author{
Faculty of Medicine \\ Department of Medicine \\ Hematology \\ Cancer Research Center (IBMCC-CSIC-USAL)
}

New insights into plasma cell differentiation and unfolded protein response in multiple myeloma: role of DEPTOR and IRE1

Doctoral dissertation presented by

Dalia Quwaider

Salamanca

2020 

Dña. Norma Carmen Gutiérrez Gutiérrez, Profesora Asociada de la Universidad de Salamanca y Médico Adjunto del Servicio de Hematología del Hospital Universitario de Salamanca,

Dña. Ana Belén Herrero Hernández, Profesora Ayudante Doctor del Departamento de Medicina de la Universidad de Salamanca,

y

D. Marcos González Díaz, Catedrático de la Universidad de Salamanca y Jefe del Servicio de Hematología del Hospital Universitario de Salamanca,

\section{CERTIFICAN}

Que el trabajo realizado bajo nuestra dirección y tutoría por Dña. Dalia Quwaider titulado "New insights into plasma cell differentiation and unfolded protein response in multiple myeloma: role of DEPTOR and IRE1", reúne las condiciones de originalidad y calidad científica requeridas para optar al Grado de Doctor por la Universidad de Salamanca.

Y para que así conste a los efectos oportunos, firmamos el presente certificado en Salamanca, a 23 de octubre de 2020.

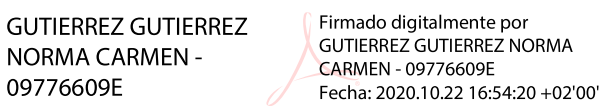

Dra. Norma C Gutiérrez

Prof. Ana Belén Herrero

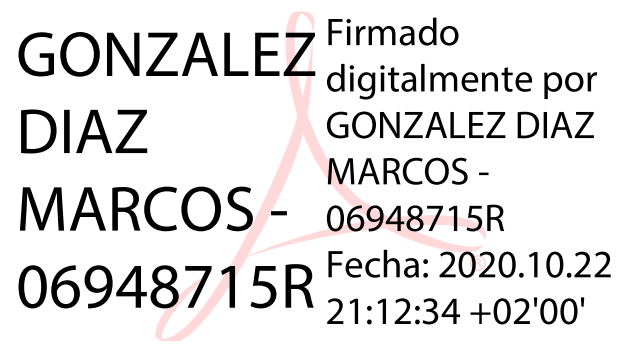

Prof. Marcos González
$06948715 R_{\text {21:12:34 +02'00' }}^{\text {Fecha: } 2020.10 .22}$
Firmado por HERRERO HERNANDEZ ANA BELEN 07961773R el día $23 / 10 / 2020$ con un certificado emitido por AC FNMT Usuarios
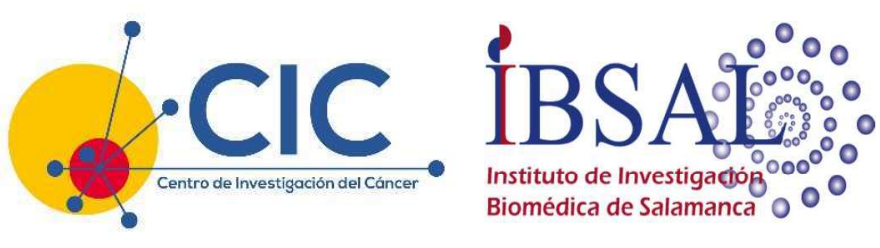

A mi madre

A mí padre 


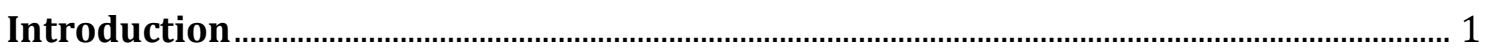

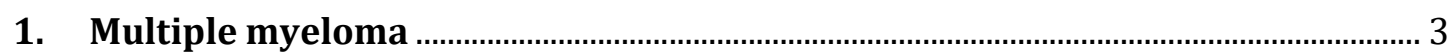

1.1. Diagnostic criteria for multiple myeloma ............................................................. 4

1.2. Genetic abnormalities in multiple myeloma ….................................................... 5

1.2.1. Chromosomal translocations …………………………………………………....... 5

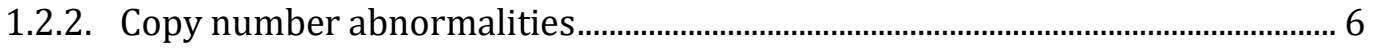

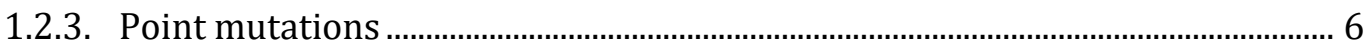

1.3. Associations between genomic abnormalities ................................................... 7

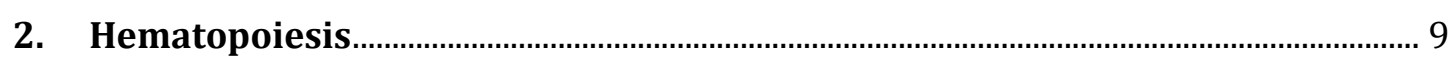

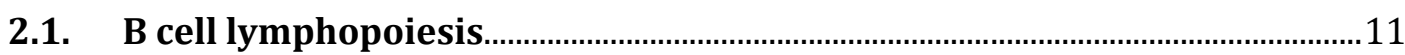

3. Regulation of B cell development ………………..................................................... 16

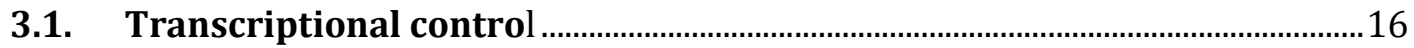

3.1.1. Transcription factors that maintain B cell fate ..................................................16

3.1.2. Drivers of PC differentiation. ................................................................................18

3.2. Unfolded protein response and its activation during $B C$ differentiation. 20

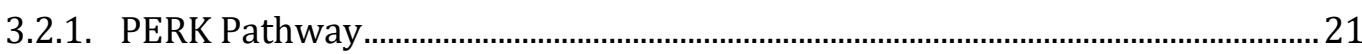

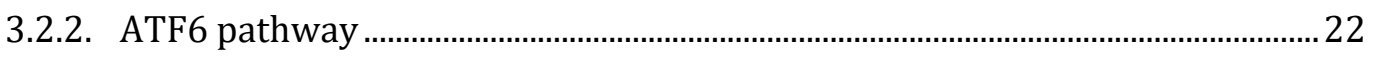

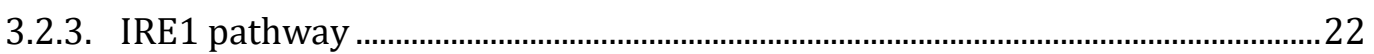

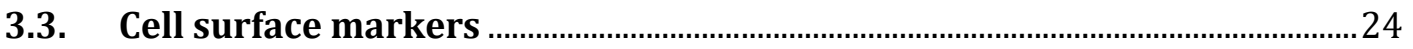

3.4. Epigenetics and $B$ cell development ………............................................................ 25

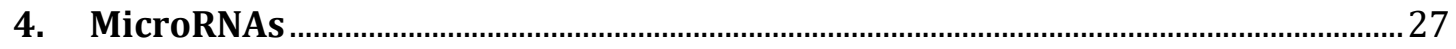

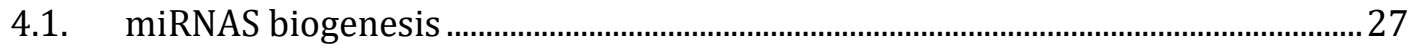

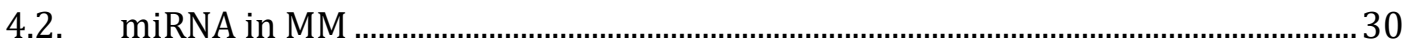

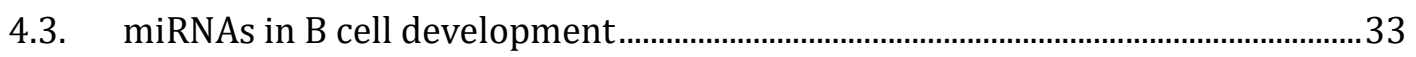

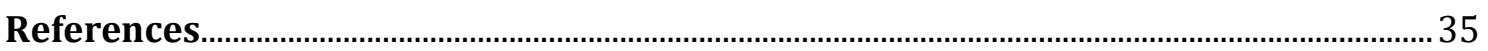

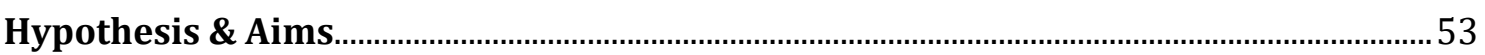

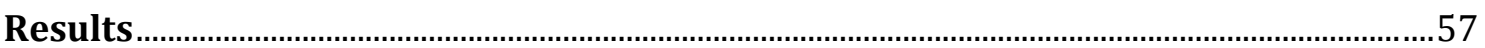

Chapter I: DEPTOR maintains plasma cell differentiation and favorably affects prognosis in multiple myeloma

Chapter II: MiR-124 and miR-506 overexpression promote plasma cell dedifferentiation through the regulation of IRE1.

Chapter III: RNA sequencing identifies novel regulated IRE1-dependent decay targets that affect multiple myeloma survival and proliferation ..................................................... 115

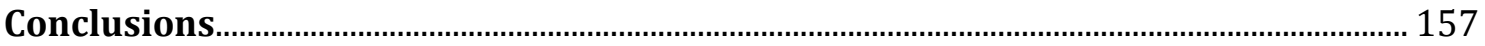

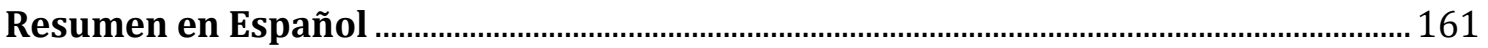

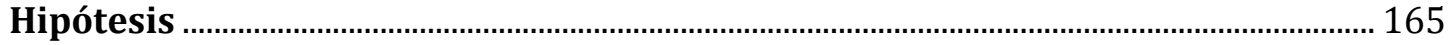


Objetivos

Resultados

Conclusiones

Anexo 


\section{List of Abbreviations}

ATF4

ATF6

B lymphocyte

BACH2

BCR

BCs

BiP

BM

BMM

BSAP

CCND1/2/3

CDRs

CHOP

CNA

c-myb

c-Myc

D

DCs

DGCR8

DLBCL

Drosha

EBF

Eif2 $\alpha$

ER

ERAD

FDC

FISH

FGFR3

F0X01

GAPDH

GRP78
Activating transcription factor 4

Activating transcription factor 6

Bursa derived lymphocyte

BTB and CNC homologue 2

B cell receptor

B cells

Binding immunoglobulin protein

Bone marrow

Bone marrow microenviroment

B cell specific activation protein

Cyclin D1/2/3

Complementary determining regions

C/EBP-homologous protein

Copy number alterations

v-myb myeloblastosis viral oncogene homolog (avian)

v-myc myelocytomatosis viral oncogene homolog (avian)

Diversity region gene

Dendritic cells

DiGeorge syndrome critical region gene 8

Diffuse large B cell lymphoma

Drosha, ribonuclease type III

Early B cell factor

Eukaryotic translational initiation factor 2

Endoplasmic reticulum

ER-associated degradation

Follicular dendritic cells

Fluorescent in situ hybridization

Fibroblast growth factor receptor 3

Forkhead Box 01

Glyceraldehydes 3-phosohate dehydrogenase

Glucose-regulated protein GRP78 


\begin{tabular}{|c|c|}
\hline HCs & Heavy chains \\
\hline HSCs & Hematopoietic stem cells \\
\hline HSPCs & Hematopoietic stem and progenitor cells \\
\hline Ig & Immunoglobulin (Ig) \\
\hline IGH & Immunoglobulin heavy chain \\
\hline IGL & Immunoglobulin light chain \\
\hline IL6 & Interleukin 6 \\
\hline IMWG & International Myeloma Working Group \\
\hline IRE1 & Inositol requiring enzyme \\
\hline IRF4 & Interferon-regulatory factor 4 \\
\hline $\mathbf{J}$ & Joining region gene \\
\hline KRAS & v-Ki-ras2 Kirsten rat sarcoma viral oncogene homolog \\
\hline LCs & Light chains \\
\hline MAF & v-maf musculoaponeurotic fibrosarcomoncogene homolog \\
\hline MALT & Mucosa-associated lymphoid tissue \\
\hline MGUS & Monoclonal gammopathy of undetermined significance \\
\hline miRNA & MicroRNAs \\
\hline MITF & Microphthalmia-associated transcription factor \\
\hline MM & Multiple myeloma \\
\hline MMSET & Multiple myeloma SET-domain containingprotein \\
\hline mRNA & Messenger RNA \\
\hline MTA3 & Metastasis associated 1 family member3 \\
\hline NK lymphocyte & Natural killer lymphocyte \\
\hline NRAS & Neuroblastoma RAS viral (v-ras oncogene homolog) \\
\hline PAX5 & Paired box protein 5 \\
\hline PB & Peripheral blood \\
\hline PCL & Plasma cell leukemia \\
\hline PCs & Plasma cells \\
\hline PERK & PKR- like ER kinase \\
\hline PI3K & Phosphoinositide 3-kinase \\
\hline pPCL & Primary plasma cell leukemia \\
\hline PRDM & Positive regulatory domain containing 1 \\
\hline PTEN & Phosphatase and tensin homolog \\
\hline RAG1/2 & Recombination activating gene \\
\hline
\end{tabular}


RISC

SHM

SMM

SPCL

T lymphocyte

TCs

TFs

TFhcs

UPR

V

XBP1

XBP1s

XBP1u

к

$\lambda$
RNA interfering silencing complex

Somatic hyper mutation

Smoldering multiple myeloma

Secondary plasma cell leukemia

Thymus derived lymphocytes

$\mathrm{T}$ cells

Transcription factors

T Follicular helper cells

Unfolded protein response

Variable region gene

$\mathrm{X}$ box protein 1

$\mathrm{X}$ box protein 1 spliced

$\mathrm{X}$ box protein 1 unspliced

Kappa light chain

Lambda light chain 

Introduction 



\section{Multiple myeloma}

Multiple myeloma (MM) is a malignancy of terminally differentiated B cells (BCs). It is characterized by the accumulation of malignant plasma cells (PCs) in the bone marrow (BM) which secrete a monoclonal immunoglobulin (Ig) known as monoclonal protein or paraprotein. The clinical manifestations derive directly from PC infiltration and/or from the effects of the secreted monoclonal Ig, and the interactions between PC and cells from BM microenvironment. The most frequent signs of end-organ damage are bone destruction, renal insufficiency, anemia and hypercalcemia, designated by the acronym "CRAB" 1-3.

The median age at the time of diagnosis is around 65 years, and only $15 \%$ of patients are under 50 years ${ }^{4}$. Although there have been major advances in the treatment, MM remains an incurable disease with a median survival of approximately five years 5 .

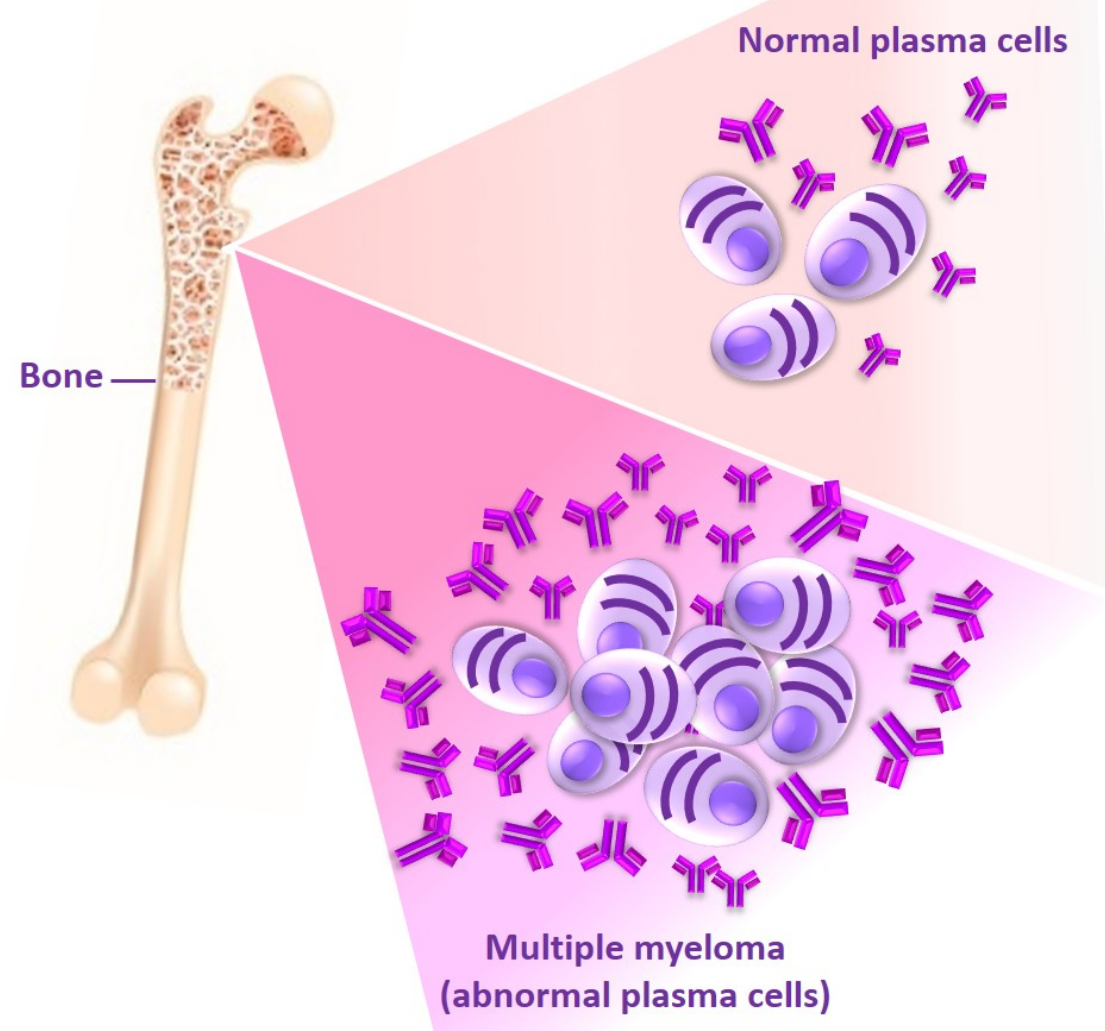

Figure 1. Normal and malignant PC. 


\subsection{Diagnostic criteria for multiple myeloma}

MM is part of a spectrum of clonal PC disorders called monoclonal gammopathies that include, monoclonal gammopathy of undetermined significance (MGUS), smoldering MM (SMM), symptomatic MM and plasma cell leukemia (PCL) ${ }^{6}$. MGUS is an asymptomatic and premalignant condition that precedes MM. It affects up to $3 \%$ of the population above the age of 50 years and progresses to $\mathrm{MM}$ at a rate of $1 \%$ per year ${ }^{7-9}$. MGUS diagnosis requires the presence of less than $0.3 \mathrm{~g} / \mathrm{L} \mathrm{M}$-protein and less than $10 \%$ of PC in the BM (Table 1). So far, it is not possible to predict whether a particular MGUS will remain benign or transform to MM since the causes of the transformation remain unclear 10-12. SMM is an intermediate, asymptomatic, but more advanced premalignant stage than MGUS. The risk of progression to MM in the first 5 years following diagnosis is higher in SMM than in MGUS, 10\% per year 13 . PCL is the most aggressive PC neoplasm characterized by the presence of circulating PCs ${ }^{14}$. The diagnosis of MM is established according to the criteria published by the International Myeloma Working Group (IMWG), which requires the presence of one or more myelomadefining events (MDE) defined as the established four CRAB features and the three myelomadefining biomarkers (Table 1) 3,15

Table 1. Criteria for diagnosis and classification of MGUS, SMM, and MM, accrding to IMWG. Modified from Rajkumar SV et al ${ }^{16}$.

\begin{tabular}{|c|c|c|c|}
\hline \multicolumn{2}{|l|}{ MGUS } & SMM & MM \\
\hline \multicolumn{2}{|c|}{ - Serum monoclonal protein $<3 \mathrm{~g} / \mathrm{dL}$} & - Serum monoclonal protein $\geq 3 \mathrm{~g} / \mathrm{dL}$ & - Serum monoclonal protein present or absent \\
\hline \multicolumn{2}{|l|}{ - $\mathrm{BMPC}<10 \%$} & - BMPC: $10-60 \%$ & - $\mathrm{BMPC} \geq 10 \%$ or biopsy-proven bony extramedullary plasmacytoma \\
\hline \multicolumn{2}{|l|}{ - No MDE } & - No MDE & - $\mathrm{MDE}$ \\
\hline \multicolumn{4}{|c|}{ CRAB features } \\
\hline Hypercalcemia: & \multicolumn{3}{|c|}{ serum calcium $>0.25 \mathrm{mmol} / \mathrm{L}(>1 \mathrm{mg} / \mathrm{dL}$ ) higher than the upper limit of normal or $>2.75 \mathrm{mmol} / \mathrm{L}(>11 \mathrm{mg} / \mathrm{dL})$} \\
\hline Renal insufficiency: & \multicolumn{3}{|c|}{ creatinine clearance $<40 \mathrm{ml}$ per min or serum creatinine $>177 \mu \mathrm{mol} / \mathrm{L}$ ( $>2 \mathrm{mg} / \mathrm{dL}$ ) } \\
\hline Anemia: & \multicolumn{3}{|c|}{ hemoglobin value of $>20 \mathrm{~g} / \mathrm{L}$ below the lower limit of normal, or a hemoglobin value $<100 \mathrm{~g} / \mathrm{L}$} \\
\hline Bone lesions: & \multicolumn{3}{|c|}{ one or more osteolytic lesions on skeletal radiography, $\mathrm{CT}$, or PET-CT } \\
\hline
\end{tabular}

\section{MDEs: Myeloma-defining events}

CRAB features (one or more ) or at least one of the following biomarkers of malignancy:

- Clonal BMPC percentage of $\geq 60 \%$

- Involved/uninvolved serum free light chain ratio of $\geq 100$

- $>1$ focal lesions on MRI studies

BMPC: Bone marrow plasma cell

CT: Computed tomography scan

PET-CT: $={ }^{18} \mathrm{~F}$-fluorodeoxyglucose PET (Positron emission tomography) with CT

MRI: Magnetic resonance imaging 


\subsection{Genetic abnormalities in multiple myeloma}

MM presents a great heterogeneity in terms of clinical course and response to treatment 17,18 , which is closely related to the genetic features of myeloma cells. Unlike what happens in particular types of leukemia and lymphoma, no specific chromosomal or genetic abnormalities have been found in MM, although a significant number of recurrent cytogenetic lesions with important prognostic impact have been described. This DNA complexity, both at genetic and chromosome levels, is also manifested in the transcriptome.

On the other hand, there is increasing evidence of the strong dependence of myeloma cells on BM microenvironment and of the influence of the immune system in the control of the disease.

Initial studies using conventional cytogenetics and specially fluorescent in situ hybridization (FISH) on purified PCs have revealed some of the most recurrent chromosomal abnormalities present in MM. Recently, modern genomic technologies based on microarray and next generation sequencing (NGS) have contributed to shape the genomic landscape of MM. The genetic abnormalities observed in MM can be categorized into translocations, copy number abnormalities (CNA) and point mutations.

\subsubsection{Chromosomal translocations}

Translocations involving the immunoglobulin heavy chain $(I G H)$ gene, located at locus $14 \mathrm{q} 32$, to different regions of the genome are observed in up to $60 \%$ of MM ${ }^{19}$. The consequence of the resulting fusion product is a deregulation of the oncogenes that are placed under the control of the IGH enhancer. The most frequent translocations are the $t(11 ; 14)$, detected in $15-20 \%$ of the cases, which increases CCND1 expression; the $\mathrm{t}(4 ; 14)$ that appears in approximately $15 \%$ of the MM patients resulting in the simultaneous deregulation of two genes, FGFR3 and MMSET/NSD2; and the $\mathrm{t}(14 ; 16)$, observed at most in $5 \%$ of MM, which leads to an increase in $M A F$ oncogene expression. Both $\mathrm{t}(4 ; 14)$ and $\mathrm{t}(14 ; 16)$, which are undetectable in the conventional karyotype, are included in the majority of prognostic stratifications as genetic markers of poor prognosis ${ }^{20}$. However, the introduction of proteasome inhibitors in the therapeutic schemes has improved the survival of patients with these translocations. In addition to IGH rearrangements, MYC oncogene has been found to be translocated in $15 \%$ of MM cases. MYC translocations mostly involve an Ig locus, although about $40 \%$ of them affect other partners ${ }^{21}$. Modern ultrasequencing techniques have detected rearrangements of $M Y C$ to a wide variety of "superenhancers" that induce a $M Y C$ overexpression in up to half of the MM cases 22 . 


\subsubsection{Copy number abnormalities}

Gains and losses of complete chromosomes or chromosomal regions are common genetic events in myeloma cells, which makes that almost all MM cases are aneuploid. Hyperdiploid MMs are characterized by the presence of trisomies, particularly involving the odd chromosomes, and a low frequency of structural alterations. The most affected chromosome by gains and losses is the chromosome 1, in which q arm is gained in $60 \%$ of cases and regions of $1 \mathrm{p}$ are lost in approximately $30 \%{ }^{23}$. Both abnormalities have been associated with short survival, but in the case of $1 \mathrm{q}$ abnormalities, amplification, defined as the presence of more than three copies of 1q, entails a worse prognosis ${ }^{24}$. Other frequent CNAs include chromosome 13 monosomy/deletion and 17p deletion. Indeed, 17p13 deletion (that include the TP53 locus), detected in $8-10 \%$ of the cases, confers a very poor prognosis ${ }^{25}$. The frequency of TP53 loss is much higher in patients with advanced MM and, in fact, is associated with extramedullary disease.

\subsubsection{Point mutations}

Whole exome and genome sequencing by next generation sequencing (NGS) of thousands of MM patients has confirmed the mutational heterogeneity of this disease. Modern strategies of massive sequencing have detected about 35 non-silent mutations per MM genome, a number that is higher than that observed in acute leukemia and much lower than the hundreds of mutations present in some solid tumors ${ }^{7}$. Unlike other hematological malignancies, a single and specific point mutation has not been found in $\mathrm{MM}$, however, several recurrently mutated genes have been identified. The genes mutated in more than $5 \%$ of patients with MM that have been consistently reported using NGS are: KRAS (20-25\%), NRAS (20-25\%), TP53 (8-15\%), DIS3 (11\%), FAM46C (11\%), BRAF (6-15\%), TRAF3 (3-6\%), ROBO1 (2-5\%), EGR1 (4-6\%), SP140 (5-7\%) and FAT3 (4-7\%) 26-31. Although the pathogenic role of RAS and TP53 mutations is well known, the effect of mutations involving the other genes is still unclear ${ }^{32-34}$. Our group has recently described that FAM46C inactivation promotes cell migration in MM ${ }^{35}$. Considering biological pathways instead of individual genes, mutations involving RAS/MAPK (KRAS, NRAS and $B R A F$ ), NFkB (TRAF3, CYLD and $L T B$ ) and DNA repair (TP53, ATM and ATR) pathways are present in 40\%, 20\% and 15\% of MM cases, respectively. Genes involved in B cell differentiation, such as PRDM1, IRF4 and SP140, are also recurrently mutated in MM. 


\subsection{Associations between genomic abnormalities}

The increasingly comprehensive genomic studies of large series of MM patients have allowed us to find significant associations among the different genomic lesions. Hyperdiploid MM cases are associated with NRAS mutations and, to a lesser extent, with $M Y C$ translocations and ERG mutations. On the other hand, hyperdiploidy and $I G H$ translocations are mutually exclusive in the vast majority of patients, although they may coexist in a small fraction of cases. Nonhyperdiploid MMs are characterized by a very high prevalence of IGH translocations and chromosome 13 monosomy, and they are associated with shorter survival. Regarding IGH translocations, $\mathrm{t}(11 ; 14)$ is associated with KRAS, IRF4 and CCND1 mutations, while $\mathrm{t}(4 ; 14)$ cases are enriched for FGFR3, DIS3 and PRKD2 mutations. Mutations in KRAS and NRAS do not usually appear simultaneously and when they coexist in the same tumor, it is almost always at subclonal level ${ }^{36}$ (Figura 2).

The coexistence of more than one high-risk genetic abnormality implies a significant shortening of survival compared with the presence of one of these lesions. Thus, MM patients with a high-risk $I G H$ translocation, a 17p deletion and a 1q gain have a median overall survival as short as 9 months. Likewise, biallelic inactivation of TP53 due to the simultaneous presence of mutation in one allele and deletion in the other is associated with very poor prognosis 24 . 
Initiation

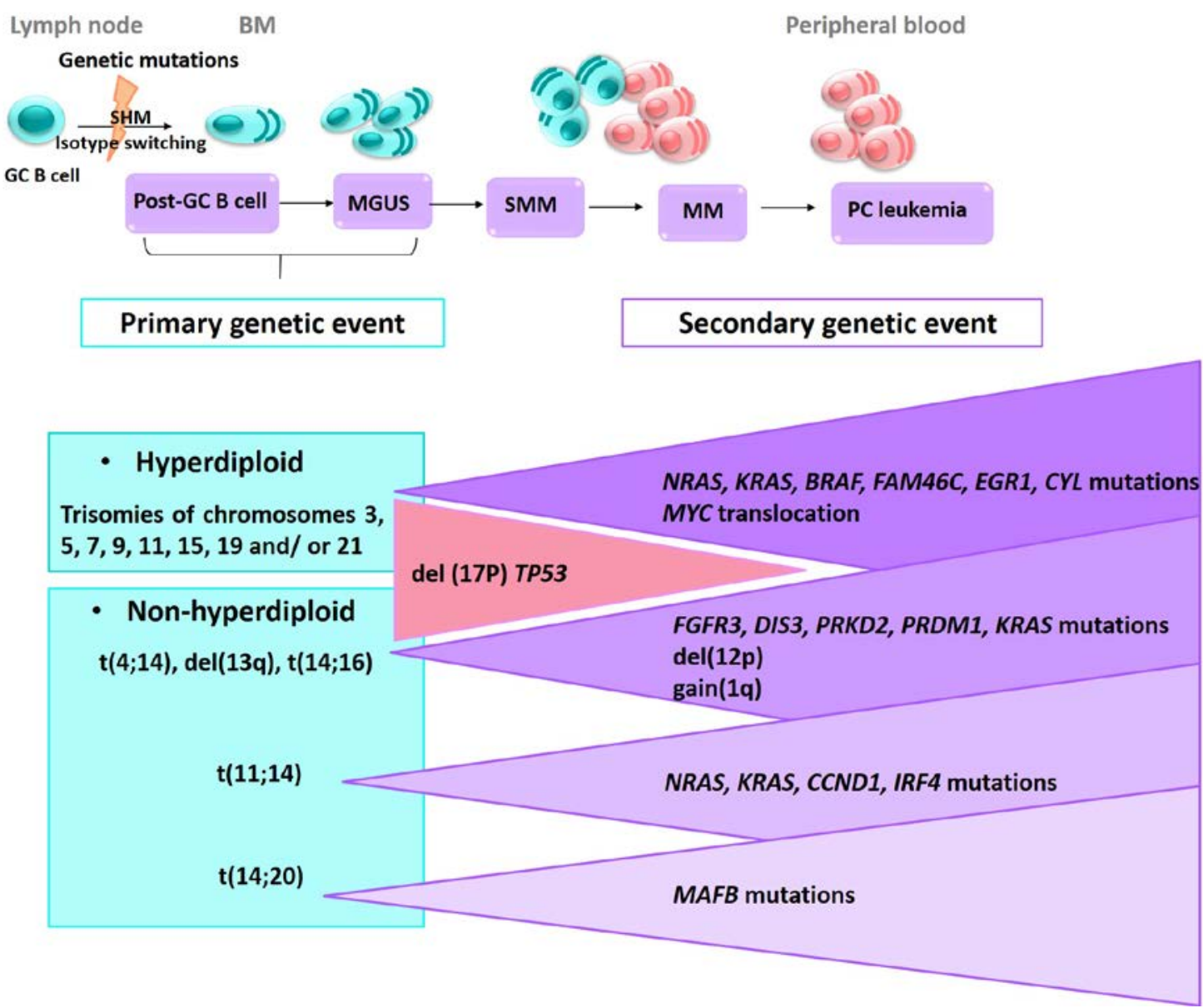

Figure 2. Pathogenesis of MM. The development of MM is a multistep molecular process, which starts with precursor disease entities, such as MGUS, SMM ending up with extramedullary myeloma/PC leukemia. Primary genetic events in the development of MGUS, SMM and MM include chromosomal translocations involving IGH and aneuploidy. The number of secondary alterations increases from MGUS to SMM and then to MM. Adapted from Kumar SK et al and Manier S et al ${ }^{3,36}$. 


\section{Hematopoiesis}

The blood system contains different cell lineages that are involved in both innate and adaptive immune system. All blood cell types arise from hematopoietic stem cells (HSCs) that reside in adult mammals mainly in the bone marrow (BM) ${ }^{37}$.

Hematopoiesis has been divided into myelopoiesis, the production of myeloid lineage cells, and lymphopoiesis, the production of lymphoid lineage cells. These cells are generated through critical stages of differentiation, from HSCs to multipotent early progenitors, which upon progressive loss of self-renewal capacity, transform into progenitors that gradually lose certain lineage differential potential, and commit to specific lineage fates and gain specialized functions. Prior to reach maturity, an early option of commitment of the differentiating cells is to develop into a common myeloid progenitor or common lymphoid progenitor ${ }^{38-42}$ (Figure 3).

$\mathrm{BM}$ is the most important myeloid organ involved in the formation of erythrocytes (erythropoiesis), granulocytes (granulocytopoiesis), monocytes (monocytopoiesis) and platelets (megakaryocytopoiesis). On the other hand, BM and thymus are the two primary organs in charge of the formation of B and T lymphocytes, whereas spleen, lymph nodes, tonsils, Peyer's patches and mucosa-associated lymphoid tissue (MALT) are considered secondary organs. MALT is a diffused lymphoid organ, which includes gut, bronchial, nasopharinx, larynx, eye, vascular- and skin-associated lymphoid tissues. Function of MALT is to ensure the complete immune response after local stimulation through both, $\mathrm{B}$ and $\mathrm{T}$ lymphocytes. 


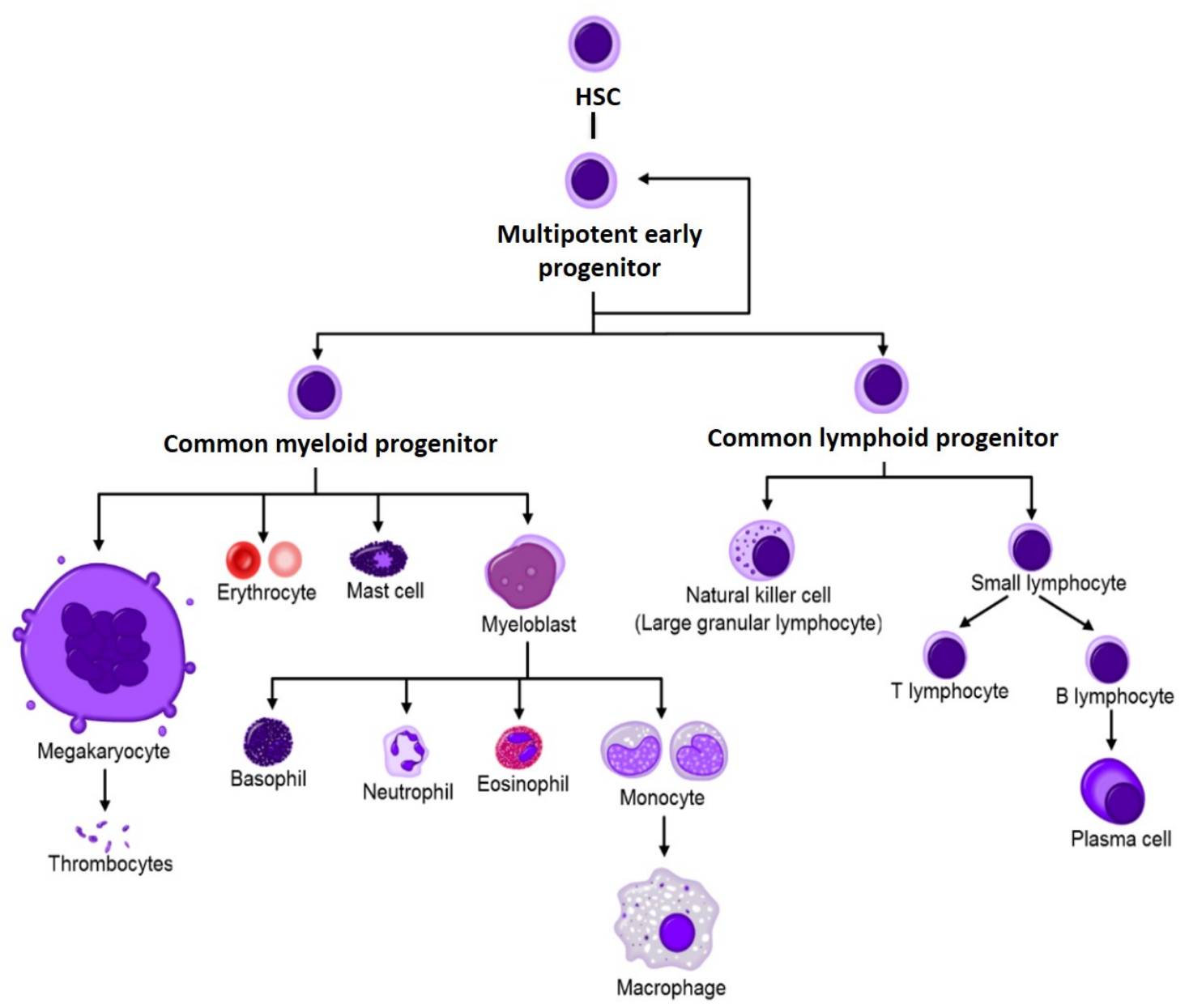

Figure 3. Hematopoietic stem cell differentiation. Schematic representation of the production of mature blood cells by the proliferation and differentiation of HSCs. Modified from Shaikh A.et al ${ }^{43}$. 


\subsection{B cell lymphopoiesis}

Lymphopoiesis or lymphoid hematopoiesis is the generation of lymphocytes, immunologically competent cells, which assist the phagocytes in the defense of the body against infection and other foreign invasions. Lymphocytes are essential for both cell-mediated and antibodymediated immunity.

The lymphocyte compartment in mammals consists of three distinct cell lineages that include bursa-derived lymphocyte (B cells) 44,45, thymus-derived lymphocytes ( $\mathrm{T}$ cells) and natural killer (NK) lymphocytes ${ }^{46}$ (Figure 3). B cells (BCs) are genetically programmed to encode a surface receptor specific for a particular antigen. Once the specific antigen has been recognized, $\mathrm{BCs}$ multiply and differentiate into plasma cells (PCs), which are terminally differentiated BCs that produce large amounts of antibodies. Antibody production is one of the most important components of the immune response.

Plasma cell (PC), also known as antibody secreting cell, is estimated to secrete several thousand antibody molecules per second ${ }^{47}$. An antibody is an immunoglobulin (Ig) protein, which is typically a Y-shaped molecule that consists of two heavy chains (HCs) encoded by the IGH locus (chromosome 14), and two light chains (LCs), encoded by either the IGL (chromosome 2) or IGK (chromosome 22) loci, linked by disulfide bonds 48 (Figure 4). The structure of the antibody molecule has been solved to an atomic level 49,50 . The two identical antigen recognition and binding sites in the molecule are made up of HC and a LC. These areas of the antibody molecule are called the variable regions ${ }^{51}$. The hypervariable tips of these regions contribute to generate a vast repertoire of slightly different antibodies. As these areas fit to an antigen shape, they are also called complementary determining regions (CDRs). The other parts of the molecule, called constant regions, preserve the structure of the molecule and are not subject to much variation. 


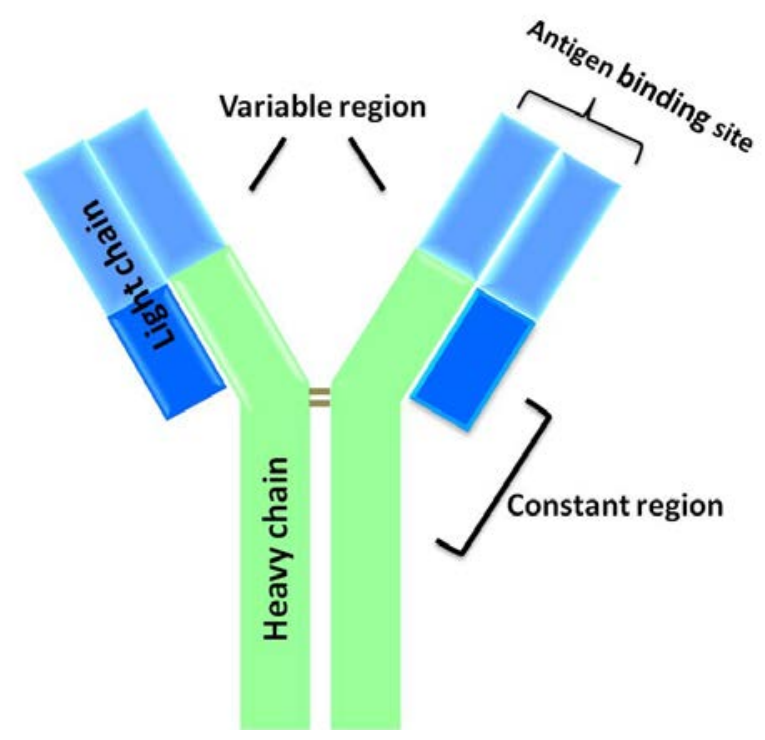

Figure 4. Schematic design of an immunoglobulin.

To generate functionally differentiated PCs, BCs undergo a series of changes from early BC progenitor until the generation of antibody producing PC. The different stages of BC development have a particular signature at genetic, molecular and morphological levels, which includes the expression of cell surface markers, increase in cell size and localization of the cells within the BM, the spleen, or the lymph nodes 52,53 . The most remarkable change that defines the differentiation process is the rearrangement of Ig genes, allowing the diversity of antibody repertoires, which is basically generated by two stages of genetic mechanisms that involve recombination and somatic hypermutation (SHM) of Ig genes 54 .

The development of $\mathrm{BC}$ receptor (BCR) takes place in the $\mathrm{BM}$ and is produced during the initial stages of $\mathrm{BC}$ development (lymphoid progenitors, pro-B and pre-B) 55 . These early stages of $\mathrm{BC}$ differentiation are antigen independent, and depend on BCR for survival 53,56. Before BCs leave the BM toward the secondary lymphatic organs, somatic DNA recombination events occur in the Ig heavy chain and Ig light chain genes (Figure 5). The IGH gene locus encodes multiple distinct copies of the variable (V), diversity (D) and joining (J) genes. The construction of the $V$ region of the heavy chain begins with the recombination of the $\mathrm{D}$ and the J genes; then the $\mathrm{V}$ gene is joined to the DJ segment. This process of site-specific recombination is highly orchestrated and mediated by the recombination activating genes $R A G 1$ and $R A G 2^{57,58}$. Finally, the constant region, known as "constant $\mu$ ", which encodes IgM antibody, is joined through RNA splicing of the primary RNA (Figure 5). On the other hand, the light chain loci ( $\operatorname{Ig} \lambda$ or $\operatorname{Ig} K \operatorname{locus}$ ) have only variable $(\mathrm{V})$ and joining $(\mathrm{J})$ gene segments. One of each gene segment is randomly selected by the RAG1/RAG2 recombinase and joined together to form the variable region 
(Figure 5). The variable light chain recombines first with the V-J segments. Next, the constant (C) domain is joined through RNA splicing of the primary RNA to the variable region 48,59 , leading to the generation of immature BCs expressing cell-surface immunoglobulin (Ig) M with kappa $(K)$, or lambda $(\lambda)$ light chains (IgM K or IgM $\lambda$ ) ${ }^{60-63}$ (Figure 5). Thus, the immature BCs leave the $\mathrm{BM}$, migrate to germinal center (GC) and undergo the $\mathrm{GC}$ reaction.

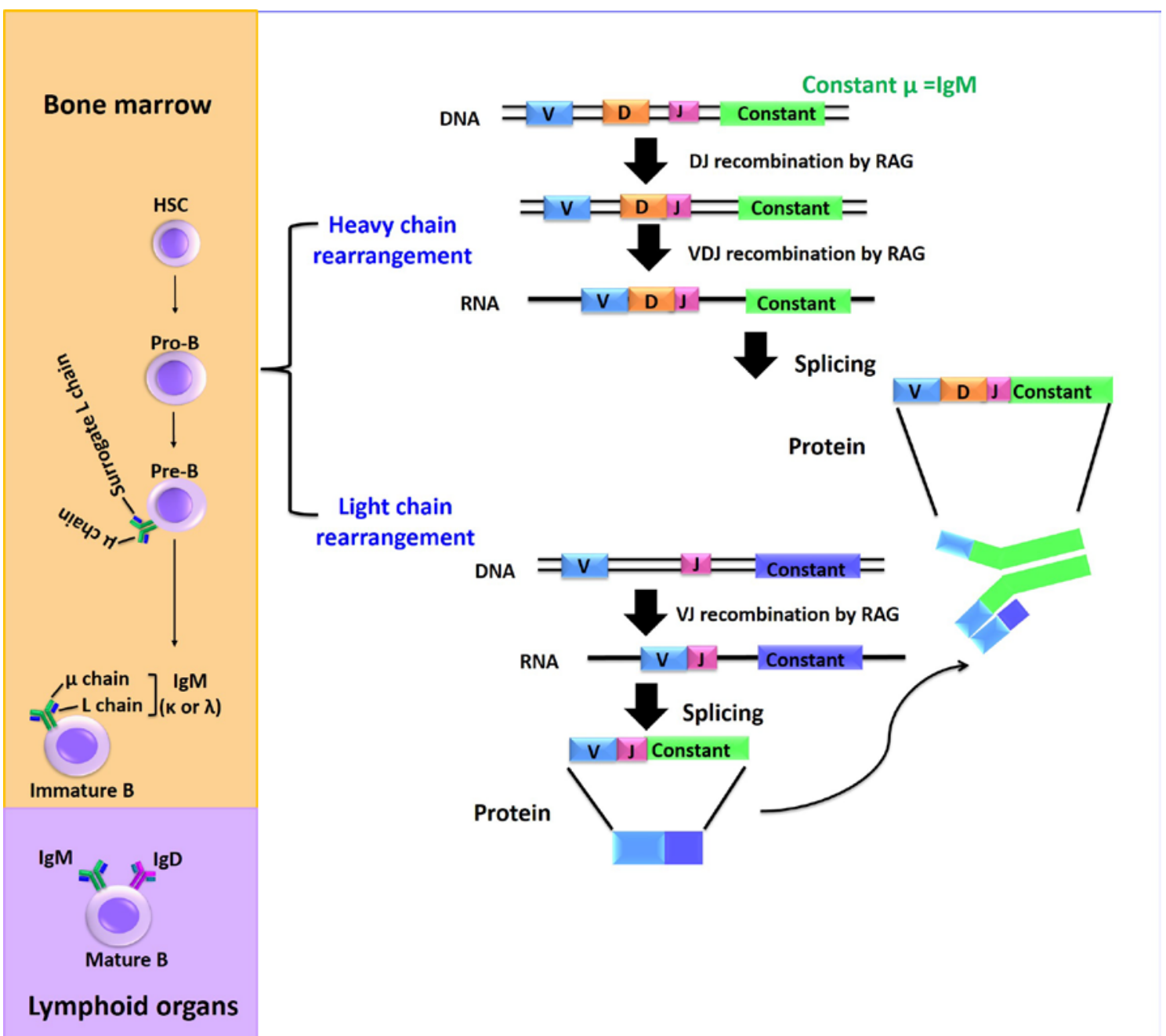

Figure 5. Development of the $\mathrm{BC}$ antigen receptor. Somatic recombination event during early $\mathrm{BC}$ development. The heavy chain gene $(\operatorname{IgH})$ undergoes a DJ recombination, followed by a VDJ recombination. Likewise, light chain genes undergo a VJ recombination. Then, surface IgM expressing $\lambda$ or $\mathrm{K}$ light chain is produced as the $\mathrm{B}$ cell receptor. Adapted from Backhaus $\mathrm{O} 48$. 
GCs are specialized microenvironments within secondary lymphoid tissues, in which BCs undergo extensive rounds of proliferation or clonal expansion, SHM, antigen-affinity driven selection, and class switch recombination (CSR) 61,64-66 (Figure 6). GC is subdivided by histologic studies in a light and a dark zone $53,61,67$.

Newly generated immature BCs that leave BM and enter the bloodstream having not yet encountered an antigen are called naive BCs. Once activated by an Ag, BCs migrate to the GC and become mature BCs expressing not only $\operatorname{IgM}$, but also IgD. Antigen-activated BCs differentiate into centroblasts that undergo clonal expansion in the dark zone of the GC. During proliferation, the process of SHM introduces base-pair changes into the V(D)J region of the rearranged genes encoding the immunoglobulin variable region $(\mathrm{IgV})$ of the heavy chain and light chain; some of these base-pair mutations lead to a change in the amino-acid sequence ${ }^{61,68-}$ 70. The activation-induced cytidine deaminase (AID) enzyme is required for SHM and CSR. During these DNA-altering processes, AID generates diversity by converting $\mathrm{C}$ to $\mathrm{T}$ and $\mathrm{G}$ to $\mathrm{A}$, indicating that AID is an essential component of both secondary immune diversification reactions ${ }^{71}$.

Centroblasts differentiate into centrocytes and move toward the light zone, where the modified antigen receptor, with the help of immune helper cells including $\mathrm{T}$ cells and follicular dendritic cells (FDCs), is selected to improve the binding to the immunizing antigen 53,72. Newly generated centrocytes that produce an unfavorable antibody undergo apoptosis and are removed, while only high affinity centrocytes that express newly generated modified antibodies are selected in the light zone, receiving proliferative and survival stimuli from FDCs and $\mathrm{T}$ follicular helper cells (TFhcs) ${ }^{72}$. These selected centrocytes will undergo CSR and differentiation into memory BCs or antibody secreting PCs. CSR is an irreversible somatic recombination mechanism by which $\mathrm{BCs}$ can switch their immunoglobulin class expression from IgM and IgD to IgG, IgA or IgE. After leaving the GC, the PCs migrate to BM where they complete their differentiation and can survive for long periods in specialized niches $67,72,73$ (Figure 6). 


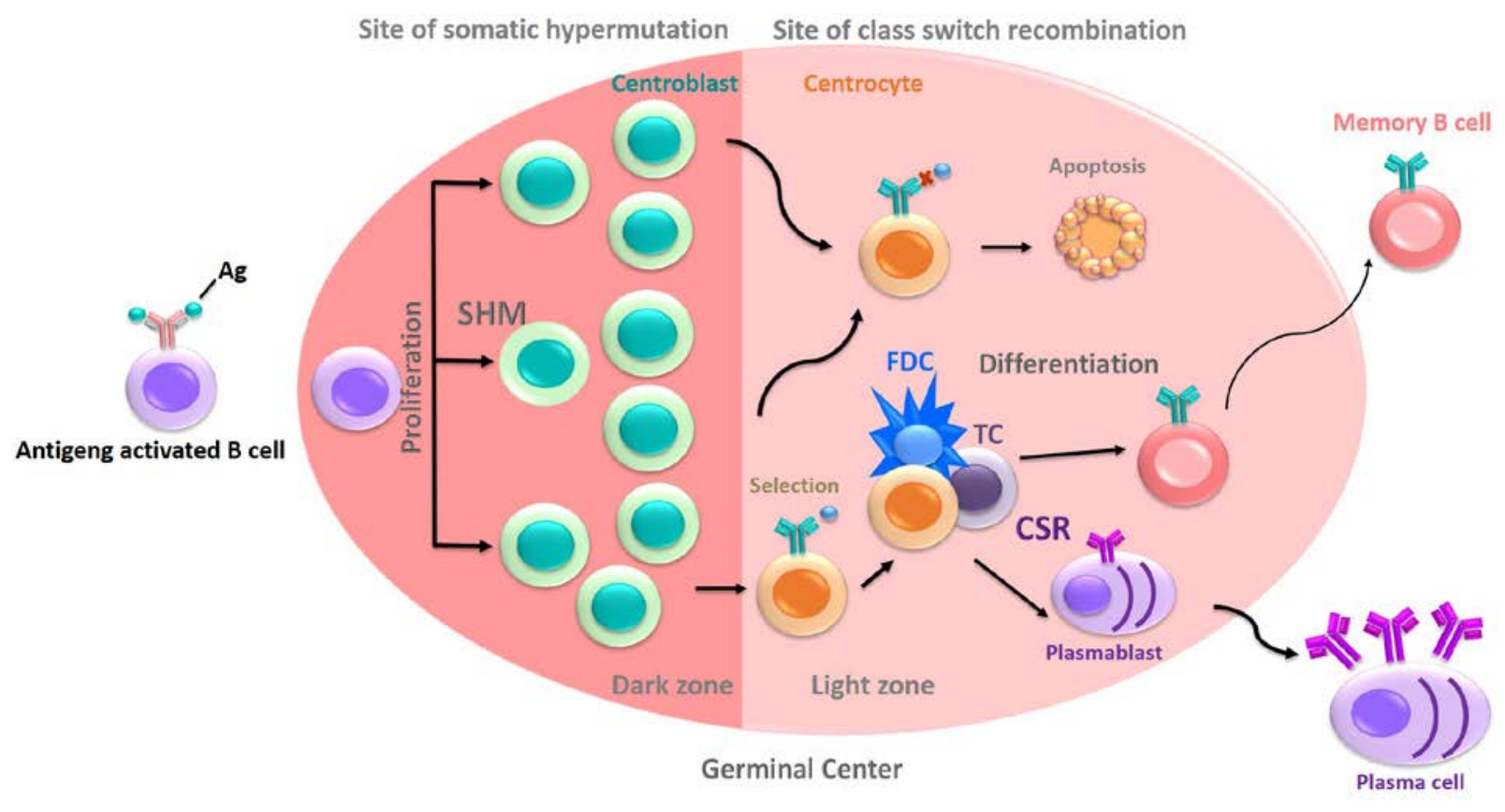

Figure 6. Germinal center reaction. Adapted from De Silva NS et al ${ }^{72}$. 


\section{Regulation of $B$ cell development}

The transition from B lymphoid precursors to PCs involves several molecular and cellular modifications including transcriptional changes, activation of biochemical pathways, expansion of the cytoplasm and the secretory organelles to accommodate high-rate synthesis of Ig, activation of the unfolded protein response (UPR), changes in cell surface markers, and also epigenetic modifications that affect the genetic program implicated in the differentiation process and cell size ${ }^{74-78}$.

\subsection{Transcriptional control}

It has been demonstrated that the transcriptome that define BC or PC status is controlled by two groups of transcription factors (TFs): those that promote BC program, and those that favor and facilitate PC differentiation. Interestingly, many of these transcription factors repress others required for the alternative developmental state, thereby establishing mutually exclusive gene expression programs ${ }^{79-81}$. When $\mathrm{BC}$ gene expression program is destabilized, and ultimately silenced, cells will commit to the PC fate by the activation of PC specific regulators (Figure 7).

3.1.1. Transcription factors that maintain $B$ cell fate. Specification and commitment of B lymphopoiesis critically depends on the expression of a number of transcription factors that promote the $\mathrm{BC}$ gene expression program and prevent premature $\mathrm{PC}$ differentiation.

TCF3 (E2A) and EBF1: the generation of the earliest BC progenitors depends on TCF3 and EBF1, which coordinately activate the $\mathrm{BC}$ gene expression program and immunoglobulin heavy-chain gene rearrangements at the onset of B-lymphopoiesis 82,83 .

PAX5 is also known as BC specific activation protein (BSAP) and is considered to be the guardian of $\mathrm{BC}$ identity ${ }^{84}$. PAX5 is expressed throughout $\mathrm{BC}$ development and is required not only for the initial lineage commitment of lymphoid progenitors to the $\mathrm{BC}$ fate ${ }^{85}$, but also for the maintenance of $\mathrm{BC}$ identity later in development ${ }^{86}$. The conditional inactivation of PAX5 in mature $\mathrm{BC}$ results in the loss of $\mathrm{BC}$ identity and in the reversion to a progenitor stage ${ }^{87}$. PAX5 has a dual function as a transcriptional activator or repressor, depending on the gene context 88. Thus, in mature BCs, PAX5 positively regulates the expression of a panel of target genes encoding proteins involved in maintaining BC identity: (i) components of the BCR, such as the immunoglobulin heavy chain (IGH) and the signal transduction chain Ig $\alpha$ (also known as CD79A); (ii) other immune receptors, such as CD19 and CD21; and (iii) transcription factors, such as interferon-regulatory factor 4 (IRF4), IRF8, BACH2 (BTB and CNC homologue 2), Aiolos 
(encoded by IKZF3 gene), SPIB, BLNK and CIITA ${ }^{89-91}$. As a transcriptional repressor, PAX5 negatively regulates a set of genes associated with antibody secreting PCs, such as X-binding protein 1 (XBP1), a transcriptional activator that is strictly required for PC development and antibody secretion 92 .

BCL6 is highly expressed in GC BCs and regulates their fate during the GC reaction by preventing terminal differentiation of BC into PC until appropriate signals are received ${ }^{93-95}$. A crucial function of BCL6 is to repress PRDM1, the gene encoding BLIMP1, which is a TF that promotes and maintains PC differentiation. Therefore, repression of BLIMP1 blocks PCs differentiation during GC reaction ${ }^{96-98}$. BCL6 promotes the rapid proliferation of GC BCs and facilitates their tolerance to the high rate of SHM through the inhibition of the DNA damage response. IRF4 and BLIMP1, two TFs that promote and maintain the differentiation of PC, have been proposed to repress the expression of BCL6 ${ }^{99,100}$.

MTA3 is a cell type-specific subunit of the corepressor complex Mi-2/NuRD that interacts directly with BCL6 to mediate the repressive activity of BCL6. When MTA3 and BCL6 are artificially increased in PC lines, the levels of BLIMP1, XBP1, SDC1 and cytoplasmic IgL ( $\kappa$ ) are reduced, promoting the reprogramming of PC maturation status into previous stages of differentiation. Therefore, MTA3 together with BCL6 has a crucial role in preventing PC differentiation 101.

MITF is a basic-helix-loop-helix-leucine-zipper protein that also inhibits PC development. It maintains mature BCs in resting state by delaying the premature differentiation of a GC BC into a plasmablast by inhibiting the expression of IRF4. It has been demonstrated that enforced expression of MITF in activated BCs represses the plasmacytoid phenotype, suppressing Ig secretion. Thus, MITF suppresses the antibody-secreting cell fate 81,102 .

BACH2 (basic leucine zipper transcription factor 2) is a transcriptional repressor required in mature and GC BCs to inhibit PC development. It is considered a crucial component of the genetic network that controls the timing of PC differentiation ${ }^{103}$. PAX5 positively induces the expression of BACH2 in early $\mathrm{BC}$ stage ${ }^{90}$, while during PC development its expression is shut down. Indeed, the main target of BACH2 is BLIMP1, which consequently remains suppressed 104,105. It has been reported that BACH2-deficient mice do not form GCs or upregulate the expression of AID ${ }^{90,103}$. Thus, in the absence of BACH2, BLIMP1 is prematurely expressed, resulting in AID repression and enhanced PC differentiation. 
3.1.2. Drivers of $\mathbf{P C}$ differentiation. The generation of $\mathrm{PCs}$ requires the suppression of $\mathrm{BC}$ transcriptional program, allowing the induction of PC transcriptional program, which is guided towards the formation of functional PCs that are capable to produce huge amount of Igs.

BLIMP1 (B lymphocyte induced maturation protein 1), encoded by PRDM1, is a transcriptional repressor that promotes the terminal differentiation of BCs into antibody secreting PCs. That is why BLIMP1 is known as the master regulator of PC differentiation 106,107. BLIMP1 negatively regulates the expression of PAX5 and BCL6 108,109, thereby ensuring that, after the induction of PC development, BCs cannot return to an earlier developmental stage. Likewise, it also represses multiple genes required for BC identity and GC function, including genes that regulate signaling through the BCR, T cell BC interactions, CSR and SHM 109. Accordingly, BLIMP1 induces the expression of many genes in PC program, such as IRF4 110,111 and genes that are involved in Ig secretion 92,107,112. Furthermore, it has been described using animal models that the inactivation of PRDM1 in PCs retains their transcriptional markers, but the capacity to produce antibodies is lost 113 .

Since BLIMP1 represses the expression of many genes to induce PC differentiation, there are many repressors that, in turn, inhibit the expression of BLIMP1 to maintain BC identity, such as BACH2, PAX5 and BCL6, which directly suppress its expression 96,101,103,114. MITF inhibits BLIMP1 indirectly by suppressing IRF4, which has been shown to be an activator of BLIMP1 $102,115,116$

IRF4 is a lymphocyte-specific member of the IRF (interferon regulatory factor) family. It is required during the immune response for lymphocyte activation and for the generation of Igsecreting PCs 116,117. IRF4 has a dual function in BC maturation, since it is essential for BC responses, such as CSR and GC BC formation, and for PC differentiation 116-119. IRF4 functions in a dose-dependent manner: high amounts of IRF4 repress BCL6, and activate both BLIMP1 and the BTB-ZF transcription factor (Zbtb20) 116,120, facilitating the PC fate; whereas low IRF4 levels promote GC generation and CSR through the activation of AID, Pou2af1 and BCL6 $116,118,121$

XBP1 (X-box binding protein) was the first TF found to be required for PC differentiation 122 . XBP1 acts downstream of BLIMP1, which upregulates XBP1 by directly repressing PAX5 92,108. XBP1 is crucial for the PC secretory program ${ }^{112}$ and its expression is induced by the activating transcription factor 6 (ATF6) ${ }^{123}$. On the other hand, XBP1 is also an essential mediator of the unfolded protein response (UPR), a signaling pathway that responds to ER stress induced by the accumulation of the unfolded proteins in the endoplasmic reticulum (ER) lumen 124. 
Expression of BLIMP or XBP1 is sufficient to drive BC differentiation towards Ig secreting PCs, although their expression is not required for PC survival 113,125. In contrast, the survival of PC critically depends on IRF4 126,127.
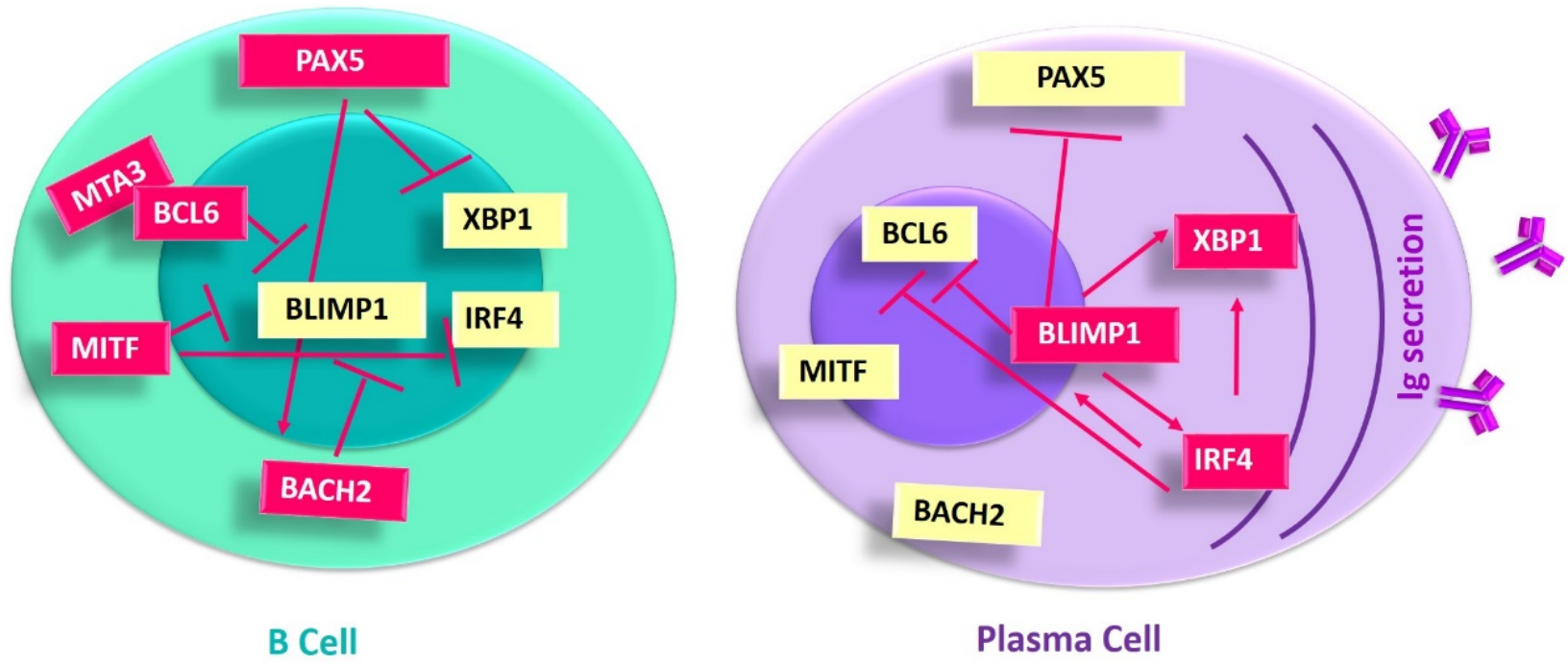

Figure 7. Transcription factors that control the transition from BC into PC. Active TFs in BC or PC are represented in red and inactive TFs in yellow. Arrows indicate transcriptional activation and T-line transcriptional repression. Adapted from Igarashi K et al ${ }^{128}$. 


\subsection{Unfolded protein response and its activation during BC differentiation}

The endoplasmic reticulum (ER) is a cellular organelle that has a variety of functions, including the facilitation of protein folding, glycosylation and the transport of synthesized proteins. Disruption of ER homeostasis triggers ER-stress. Almost all secretory and membrane proteins achieve their final folding in the ER before heading to their target organelles or the cell surface. ER stress occurs when the load of proteins exceeds the folding capacity of the ER. This situation is observed when the cells are exposed to environmental or physiological stresses such as glucose starvation, calcium depletion from the ER, strong reducing conditions, viral infection, hypoxia and also during the transition from B lymphocytes into PCs.

B lymphocytes have a limited ER and synthesize small amounts of cell-surface Ig. Upon stimulation with antigens, the activated BCs increase their secretory apparatus to accommodate synthesis of thousands of antibody molecules per second, which are efficiently assembled and secreted from its fully differentiated PC progeny. This morphological makeover requires increase in cell size and in every component of the cellular machinery for protein secretion, including ER chaperones and folding enzymes that drive assembly and maturation of Ig molecules 129,130 . The mammalian UPR is orchestrated by three transmembrane sensors: PERK, ATF6 and IRE1 131 (Figure 8). Under homeostatic conditions these sensors are bound to $\mathrm{BiP}$, a major ER chaperone that suppresses their activity 132,133. Upon ER stress, $\mathrm{BiP}$ is released from ER transmembrane sensors leading to the activation of these UPR signaling pathways ${ }^{134}$. 


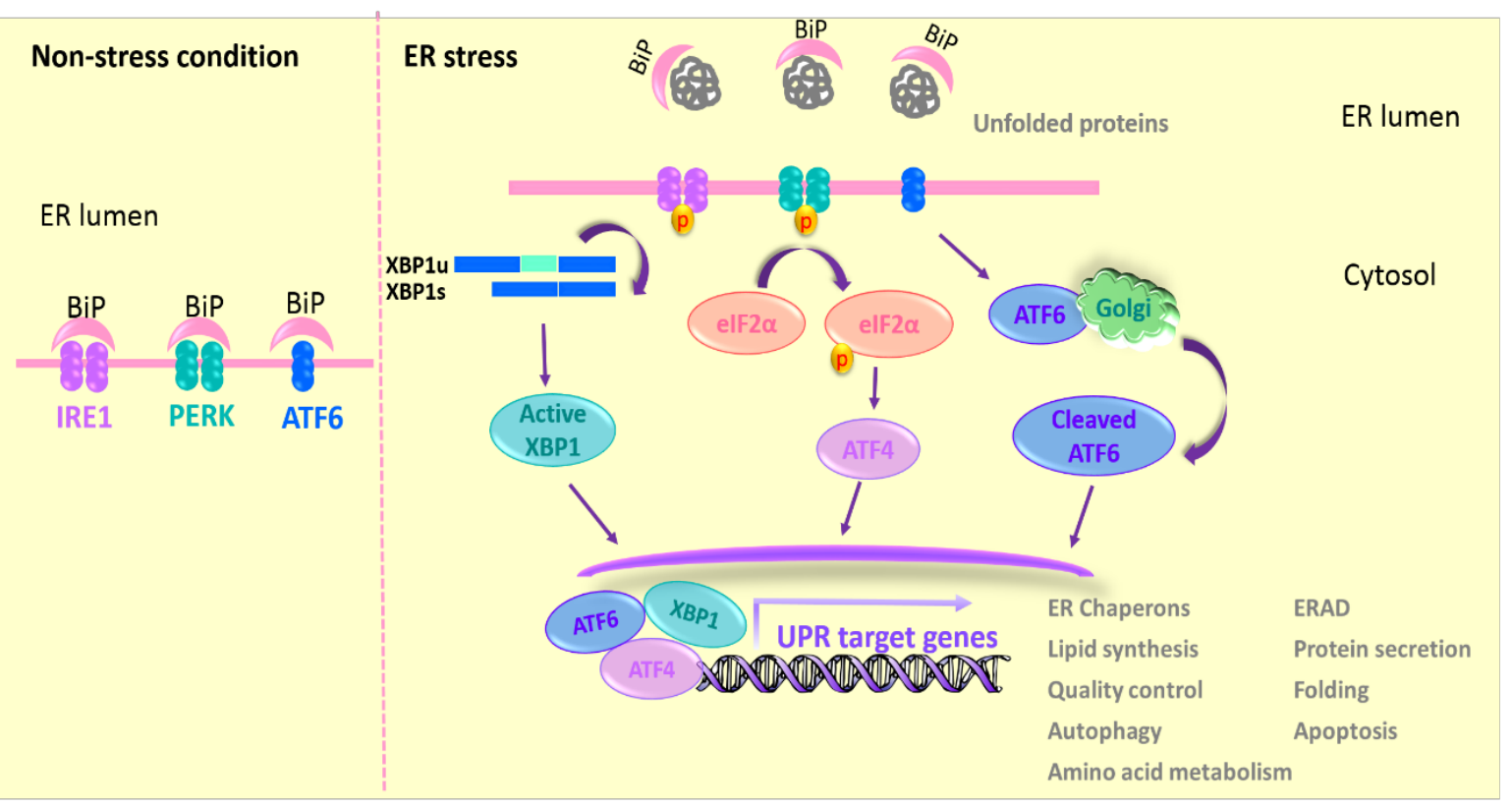

Figure 8. Unfolded protein response. In response to an increase in unfolded proteins in the ER lumen, three sensors that are located in the ER membrane, PERK, IRE1 and ATF6, activate the UPR. Under homeostatic conditions, PERK, IRE1 and ATF6 are bound by binding BiP, which suppresses their activity. In response to ER stress, BiP is recruited away from PERK, IRE1 and ATF6 to promote protein folding, which leads to the activation of these ER stress sensors. Adapted from Vincenz L et al ${ }^{135}$.

\subsubsection{PERK Pathway}

PKR-like ER kinase (PERK; official symbol EIF2AK3) is ubiquitously expressed in the body, and has two domains, an ER luminal domain and a cytoplasmic kinase domain 136,137. BiP detachment from the ER luminal domain, upon ER stress, leads to oligomerization ${ }^{132}$, transautophosphorylation and PERK activation ${ }^{138}$. PERK dimerizes to promote its autophosphorylation and activation 139. Activated PERK phosphorylates the eukaryotic translation-initiation factor $2 \mathrm{a}(\mathrm{eIF} 2 \alpha)$ to attenuate the rate of general translation initiation and prevent further protein synthesis 136,140,141. Blocking translation during ER stress consequently reduces the protein load in the ER folding machinery ${ }^{142}$. On the other hand, phosphorylated eIF $2 \alpha$ selectively activates translation of genes that have short upstream open reading frames in their $5^{\prime}$ untranslated region (5'UTR), such as the transcription factor ATF4, which upregulates genes involved in amino acid import, metabolism, and resistance to 
oxidative stress. Although ATF4 confers a cytoprotective effect in the early phase of ER stress 142,143 , it also induces the expression of C/EBP-homologous protein (CHOP), which is a proapoptotic transcription factor, in the later phase of ER stress. CHOP inhibits function of BCL2 family members, which protect cells from apoptosis, and therefore leads to cell death. Thus, the PERK pathway has opposing effects, cytoprotective or apoptotic, depending on cellular conditions.

\subsubsection{ATF6 pathway}

ATF6 functions as a bZIP transcription factor that enhances expression of ER chaperone genes. Dissociation from BiP allows ATF6 to translocate from the ER to the Golgi apparatus, where it is cleaved. The cleaved form of ATF6 migrates into the nucleus and acts as an active transcription factor to upregulate proteins that increase ER folding capacity, such as the ER chaperones, GRP78 and GRP94, and the folding enzyme, PDI. ATF6 can also induce the expression of XBP1 and CHOP to enhance UPR signaling ${ }^{144}$. However, if the disruption of the ER function cannot be resolved, ATF6 upregulates factors involved in apoptosis that initiate a programmed cell death to restore cellular homeostasis 145-148.

\subsubsection{IRE1 pathway}

Inositol-requiring enzyme-1 (IRE1; official symbol ERN1) is an ER-localized transmembrane protein that senses unfolded proteins and it is considered a master regulator of the UPR pathway. IRE1 is a bifunctional enzyme with kinase and endonuclease activities ${ }^{149}$. ER stress triggers the oligomerization and trans-autophosphorylation of IRE1 leading to the activation of its RNase activity 150,151 . The primary target of IRE1 is the mRNA that encodes the XBP1. In mammalian cells, the full length mRNA of XBP1, also referred as the un-spliced form (XBP1u), is spliced by IRE1 at two specific sites, which results in the removal of an unconventional intron of 26-nucleotide. This splicing event causes a frameshift that allows translation of the spliced form (XBP1s), which encodes a potent transcription factor ${ }^{152}$. When XBP1s is translocated to the nucleus, it induces the expression of target genes involved in protein synthesis, secretion and expansion of the secretory apparatus and cell size 112 . 

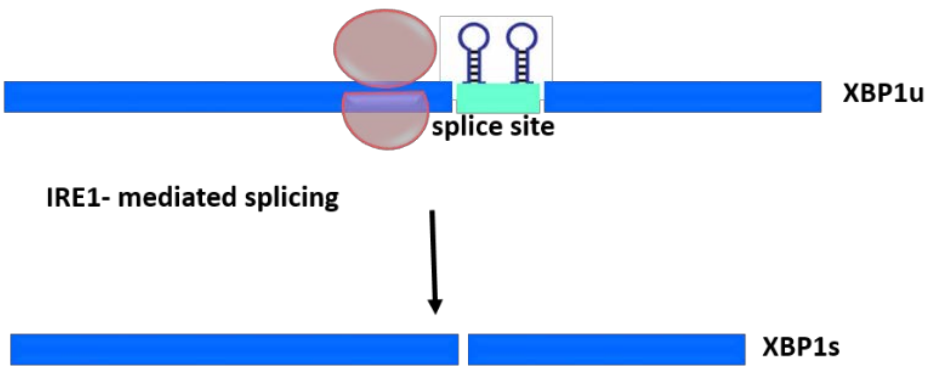

Figure 9. Schematic representation of XBP1 mRNA splicing by IRE1 during ER stress response in the ER membrane. The $26 \mathrm{nt}$ intron is spliced from XBP1u mRNA by IRE1, producing the active form XBP1s.

XBP1 is mainly known for its role in the ER stress response, targeting genes such as DnaJ, p58, ERdj4, EDEM and PDI, which are involved in protein folding and ERAD (ER-associated degradation) ${ }^{153,154}$. ERAD targets are selected by a quality control system within the ER lumen and are ultimately destroyed by the cytoplasmic ubiquitin-proteasome system (UPS) ${ }^{155}$.Thus, the UPR progresses through transient attenuation of translational and transcriptional induction of ER chaperones, folding enzymes, and proteins involved in ERAD to alleviate protein aggregation in the ER as an adaptive response. However, under severe and prolonged ER stress, the UPR activates unique pathways that lead to cell death through apoptosis ${ }^{156}$.

In summary, upon ER stress, transcriptional induction, mediated by IRE1 and ATF6, and translational regulation, mediated by PERK, are complementary for maintaining ER homeostasis and ensuring cell survival. 


\subsection{Cell surface markers}

BC differentiation is commonly presented as a linear process through sequential series of stages to end up with the production of fully functional mature cells. The maturation process along each lineage is tightly controlled by sequential expression of genes and their products 157 , as detailed in the previous sections. As BCs mature and differentiate, they give rise to multiple functionally distinct BC subsets. The diverse nature and function of these different BC subsets can be deciphered by the differential expression of cell surface and intracellular markers, as well as the distinct immunoglobulin and cytokine secretion profiles.

Cell surface markers may have diverse functions, such as growth factor receptors, adhesion molecules, extracellular enzymes, and signal transduction molecules. Some of these cell surface antigens are restricted to a specific cell lineage, while others are widely expressed along different cell lineages, however their levels depend on the maturational stage which reflect their expression patterns 158 .

The analysis of cell surface markers, such as CD10, CD19, CD20, CD21, CD24, CD34, and CD38 allows the identification of both early and late stages of development 159,160, especially in those cases where immunoglobulin cannot be used to distinguish between cell types. Thus, CD19, a signal transduction molecule, is expressed throughout $\mathrm{BC}$ development up to, but not including, the mature PC stage ${ }^{161}$. The expression of syndecan-1 (CD138) distinguishes circulating plasmablasts and PCs from other developmental and functional subsets. The key markers associated with each BC stage are summarized in (Figure 10). 


\subsection{Epigenetics and B cell development}

Epigenetic modifications include histone post-translational modifications, DNA methylation/demethylation, and alteration of gene expression by non-coding RNAs, including microRNAs (miRNAs) and long non-coding RNAs (lncRNAs). All these epigenetic changes play important roles in several biological processes, such as cell growth, apoptosis, differentiation, development, immune response and aging 162.

Epigenetic modifications, in concert with transcription factors, have been shown to play critical roles in BC development and differentiation 163,164. In fact, epigenetic mechanisms involved in the reprogramming and differentiation of BCs have been the subject of numerous studies. For instance, naive BCs display an inactive epigenetic status, showing genome-wide DNA hypermethylation and histone deacetylation 165, which makes that very few genes are expressed except those required for BC lineage, such as CD19, PAX5, EBF1 and SPIb, exhibiting active epigenetic status 166. Implication of epigenetics has also been reported in PC differentiation. Thus, BLIMP1 has been demonstrated to induce histone deacetylation in the promoter region of BCL6, PAX5, and SPIb, which display low histone acetylation levels in PCs 167. Furthermore, BLIMP1 has been found to bind to H3K9 methyltransferase G9a, therefore recruiting this enzyme to the promoter regions of SPIb and PAX5, leading to gene silencing 168 . In addition, SHR and CSR have been demonstrated to be regulated by DNA methylation and histone modification 169 .

Most studies on the role of noncoding RNAs in PCs have focused on miRNAs. miRNAs regulate gene expression through targeting the 3'UTR of mRNA transcripts, which leads to their degradation or translation inhibition 170 .

Figure 10 summarize the factors mostly implicated in the regulation of $\mathrm{BC}$ differentiation. 


\section{miRnAs}

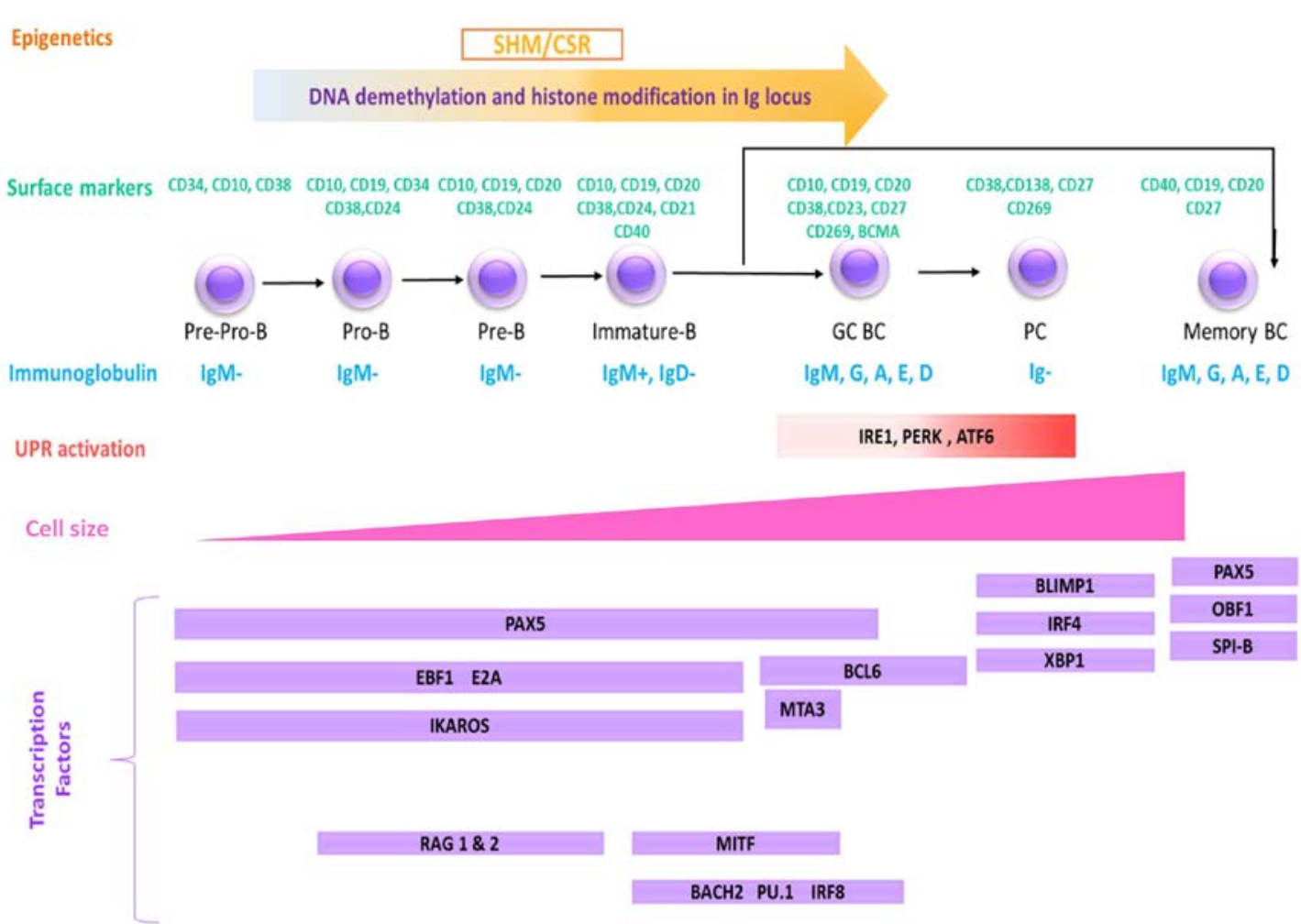

Figure 10. Summary of molecular and cellular changes throughout the process of differentiation from BC to PC. 


\section{MicroRNAs}

\section{1. miRNAS biogenesis}

MicroRNAs (miRNAs) are short non-coding single-stranded RNAs of approximately 22 nucleotides in length. They were first discovered in C. elegans ${ }^{171}$ and later in plants and animals 172. In humans, as well as in other mammalians, almost 2,300 different miRNAs have been identified ${ }^{173}$. The main function of miRNAs is the regulation of gene expression at a posttranscription level, since they bind to the 3'untraslated region (3'UTR) of target mRNAs, resulting in mRNA degradation or translation suppression 174,175 . Gene regulation mediated by microRNAs has a broad impact on gene expression, since each miRNA can control hundreds of gene targets and many mRNAs have target sites for multiple different miRNAs. This makes that miRNAs potentially control the expression of about one-third of human mRNAs ${ }^{176-180 .}$

Most human miRNA genes are located between protein-coding genes (intergenic miRNAs), while about one-third of them have been found inside protein-coding genes, called host genes 181. miRNAs are frequently grouped into clusters. Generally, there are between two and three miRNA genes in a cluster, but larger clusters have also been identified, like the miR-17 92 cluster composed of six members ${ }^{182}$.

The biologically active miRNAs are generated in a two-step sequential mechanism involving two RNase III nucleases, Drosha and Dicer. They are key factors in the biogenesis of miRNA whose downregulation has been reported in many cancer types ${ }^{183}$. Processing of the hairpin precursor (pre-miRNA) through Dicer generates a miRNA: miRNA duplex (the 5'strand and the 3'strand). The miRNA machinery is orchestrated by two major multiprotein complexes, the Drosha complex in the nucleus, and the cytoplasmatic RNA-induced silencing complex (RISC), which contains the Argonaute (Ago) family proteins as a core component (Figure 11) 172,174,184189. One of these strands, known as the guide arm, will be incorporated into RISC complex, while the other, known as the passenger strand, will be degraded. One of the two strands is preferentially selected as guide, either $5^{\prime}$ or $3^{\prime}$. While either of the miRNAs strands can be used equally, the selection of the strand is highly regulated and depends on cell/tissue type 190,191. Perfect binding of the seed sequence of the miRNA to the mRNA leads to complete mRNA degradation, while imperfect binding leads to protein translation inhibition 178,192. 


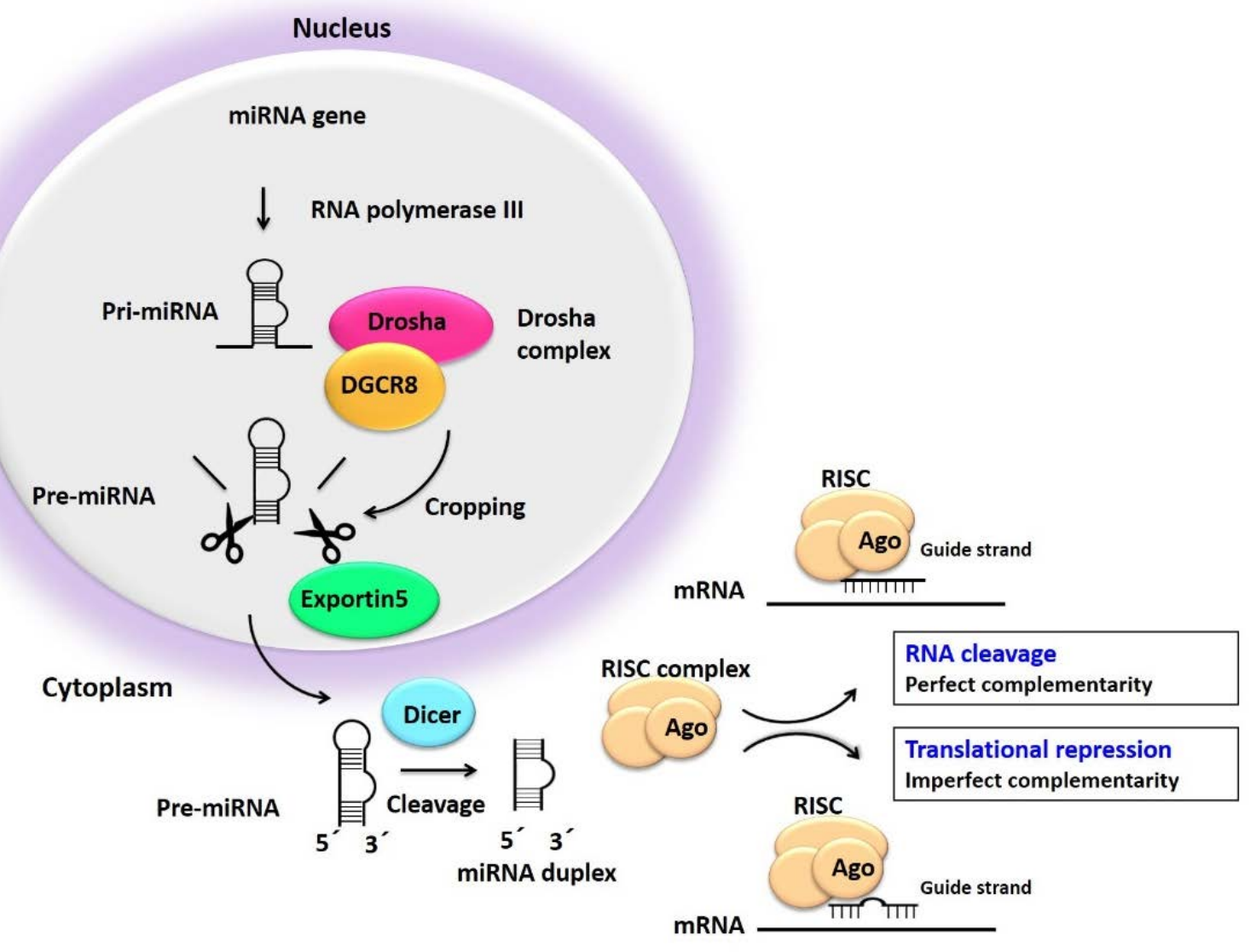

Figure 11. Schematic representation of miRNA biogenesis and function 193. 
It is well known that miRNAs are involved in critical biological processes, including cellular growth and differentiation, and in cancer pathogenesis 172,174,194-196. The miRNAs downregulated in cancer cells that prevent cancer development by inhibiting the expression of proto-oncogenes, are known as tumor suppressor miRNAs 197, while those upregulated that contribute to carcinogenesis by inhibiting tumor suppressor genes, are considered oncogenic miRNAs (oncomiRs). Whether a miRNA acts as an onco- or a tumor suppressor miRNA is highly dependent on the cellular context ${ }^{198}$.

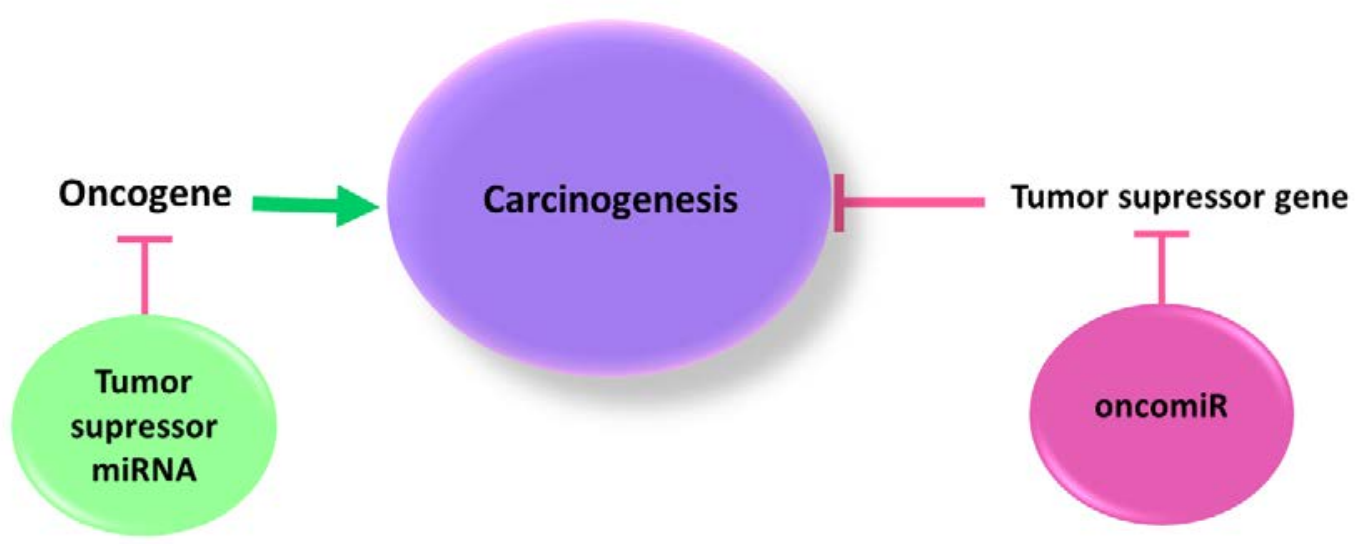

Figure 12. Schematic representation of the role of miRNA in carcinogenesis. The arrow and T-line indicate activation and repression respectively. 


\subsection{MiRNA in MM}

Over the past decade, the crucial role of miRNAs in the pathogenesis of many cancers has been clearly demonstrated. In particular, the deregulation of miRNAs in MM compared to normal PC has been reported 199,200, and several studies have revealed the involvement of miRNAs in MM pathophysiology 201-206. Moreover, the therapeutic potential of miRNA modulation has been investigated not only to target myeloma cells, but also to disrupt the functional interaction between myeloma cells and bone marrow microenvironment 207-210.

There are several factors that regulate miRNA expression and function in MM, mainly transcription factors, but also genomic and epigenetic changes in miRNA genes ${ }^{193}$. It has been demonstrated that different cytogenetic abnormalities are correlated with specific miRNA signatures (Table 3), for example, upregulation of miR-1 and miR-133 is correlated with MM bearing the $t(14 ; 16) 211$.

The implication of miRNAs in the pathogenesis of MM has been reported in several studies that demonstrate the regulation of specific genes by miRNAs, as in the case of TP53 and its expression network at multiple levels. Actually, aberrant miRNA expression leads to TP53 deregulation in MM 212. Two upregulated miRNAs in MM, miR-25 and miR-30d, were demonstrated to interact with the 3'UTR of the human TP53 gene downregulating its expression. Thus, knockdown experiments of these miRNAs in MM cells led to increased p53 expression and its downstream targets, resulting in cell apoptosis ${ }^{213}$. Additionally, the miR$125 a-5 p$ which has been described to be upregulated in a subset of MM patients carrying the $\mathrm{t}(4 ; 14)$ translocation ${ }^{214}$, directly binds to TP53 mRNA 3'UTR triggering a decrease of p53 levels. Interestingly, inhibition of miR-125b was demonstrated to overcome dexamethasone resistance in MM cells by activation of p53 downstream targets 215 .

Several miRNAs have also been described to indirectly regulate p53 by affecting some of its regulators 216,217 . MiR-34a was shown to negatively regulate SIRT1, a protein that deacetylates p53 limiting its ability to transactivate its target genes ${ }^{218}$. As a consequence of miR-34a expression, p53 pathways were activated. This miRNA could be of particular interest in MM, since it was induced in MM cells by treatment with dexamethasone, which suppressed SIRT1 deacetylase and consequently allowed p53 activation ${ }^{219}$. MiR-192, miR-194 and miR-215 have been described to target MDM2 in myeloma cells, and their ectopic expression induced significant down-regulation of MDM2, which was accompanied by p53 overexpression and p21 activation ${ }^{220}$. Interestingly, these miRNAs can, in turn, be transcriptionally activated by p53 demonstrating an autoregulatory loop between miRNAs and p53. In this regard, our group has also demonstrated that miR-214 activates p53 by targeting PSMD10 that encodes the 
oncoprotein gankyrin, which negatively regulates p53 by enhancing its proteasomal degradation ${ }^{221}$. Specifically, using gain-of-function experiments and luciferase reporter assays we observed that ectopic transfection of miR-214 decreased the level of gankyrin protein by directly targeting PSMD10 3'UTR. Moreover, as a consequence of gankyrin down-regulation in cells with WT TP53, an increase of TP53 mRNA levels and subsequent up-regulation of CDKN1A (p21Waf1/Cip1) and BAX transcripts, which are direct transcriptional targets of p53, were observed. Taken together, the reported findings suggest an important role for miRNAs in the regulation of the p53 tumor suppressor.

As in other tumors, miRNAs may also act in MM as oncomiRNAs, like miR-21, whose upregulation, demonstrated by several studies, affect cell migration, apoptosis and drug resistance ${ }^{222}$. Additionally, the miR-17 92 cluster, which encodes six miRNAs (miR-17, miR18a, miR-19a, miR-19b, miR-20a and miR-92a) all processed from the same precursor transcript, is frequently overexpressed in MM. This miR-cluster has been shown to target the proapoptotic gene BIM suggesting a possible mechanism through which overexpression of miR-17 92 could contribute to the anti-apoptotic signals in MM 199.

Conversely, several miRNAs can act as tumor suppressors in MM. For example, the ectopic expression of miR-15a and 16, both located at 13q and frequently downregulated in MM, have been shown to affect myeloma proliferation by inhibiting AKT and MAP-kinases, and by reducing bone marrow angiogenesis ${ }^{223}$. Similarly, miR-137 and miR-197, both downregulated in $\mathrm{MM}$, are considered as tumor suppressors since they mediate apoptosis in MM cells via targeting MCL-1 205. MiR-34a is one of the most characterized tumor suppressor miRNAs in several cancers, and an anti-myeloma effect has been described in in vitro and in vivo studies 224,225. Likewise, miR-155 whose reduced expression is linked to the hypermethylation of the first exon of miR-155 host gene, has also been reported to have anti-myeloma activity 226.

Finally, the impairment of proteins involved in miRNA biogenesis may lead to different miRNA expression profile in MM 193. Notably, silencing of AGO2 results in a decrease of total miRNA levels 227,228. DICER mRNA level in NPC was very similar to MGUS, and significantly higher than in SMM and MM 229. Moreover, higher expression of DICER was associated with improved progression-free survival in symptomatic myeloma patients ${ }^{229}$. Overall, these studies support the idea that the aberrant expression of proteins involved in the biogenesis of miRNAs may affect the MM pathogenesis. 
Table 2. Deregulated miRNAs in each of the cytogenetic groups of MM in comparison with NPC.All miRNAs were downregulated in myeloma cells except miR-1, miR-449 and miR-133a, which were upregulated in $\mathrm{t}(14 ; 16){ }^{211}$.

\section{Cytogenetic group Deregulated miRNAs}

$t(4 ; 14) \quad$ miR-133b

$\mathrm{t}(11 ; 14) \quad$ miR-650, miR-125a, miR-375, miR-184, miR-214, miR-95, miR-199a

$\mathrm{t}(14 ; 16) \quad$ miR-1, miR-449, miR-133a, miR-196b, miR-135b, miR-214, miR-375, miR-642

miR-196a, miR-486, miR-375, miR-501, miR-320, miR-20a, miR-133b, miR-135b, miR-126, miR-650, miR214, miR-19b, miR-10a, miR-15a, miR-133a, miR-139, miR-197, miR-10b, miR-95, miR-186, miR-19a, miR-

RB deletion $\quad 451$, miR-7b, miR-140, miR-125a, miR-362, miR-33, miR-223, miR-224, miR-221, miR-30e, miR-374, miRlet-7c, miR-99b, miR-130a, miR-193a 


\section{3. miRNAs in B cell development}

Several studies have revealed the involvement of particular miRNAs in BC development 180, 230232 (Figure 13). Here, we will mention some examples of miRNAs that are implicated in BC maturation. MiR181a was the first miRNA reported to have a role in $\mathrm{BC}$ differentiation in the BM. Moreover, it was identified as a miRNA differentially expressed in T and BCs. MiR181a ectopic expression promoted a substantial increase in the generation of BCs, both in vitro and in vivo ${ }^{233}$. In agreement with these results, miR-181a/b deficient mice showed a mild decrease in the number of peripheral and GC BCs ${ }^{234}$. Interestingly, miR-181a was later shown to play an important role in the modulation of the thresholds of TCR signaling in thymocytes 235,236, providing a nice example of a miRNA playing distinct roles in different cell lineages, presumably by affecting different sets of target genes. Additionally, miR-155, highly expressed by GC BCs, has been found to be associated with BC differentiation. In miR-155 knockout mice, reduced GC BCs and memory BCs along with decreased high-affinity IgG1 antibodies were found 237-239. MiR-155 also regulates AID expression and has a vital role in the differentiation of memory BCs 240 .

Indeed, it has also been shown that essential factors for PC differentiation, such as PRDM1, IRF4 and $X B P 1$ can be regulated by miRNAs. Thus, PRDM1, whose mRNA contains a long $(>2,000 \mathrm{nt})$ 3' UTR, can be potentially targeted by multiple miRNAs, including miR-9, miR-23b, miR-30, miR-125b, miR-127, and let-7 241-248. In fact, overexpression of miR-125b in BCs impairs BLIMP1 expression and inhibits BC differentiation into PCs ${ }^{244}$. In addition, miR-125b can downregulate IRF4, which reciprocally regulates BLIMP1 116,241,244. Furthermore, XBP1 that governs late events of PC differentiation can be downregulated by miR-127 248 .

Interestingly, the proteins involved in miRNA biogenesis are also implicated in BC development 164,231. It has been described that in an AG02-deficient hematopoietic system, B lymphogenesis is affected by a reduction in pro- $\mathrm{B}$ and specially pre-BCs ${ }^{249}$, and it has also been found that DICER ablation in progenitor BCs blocks the pro-B to pre-B transition 250 . 


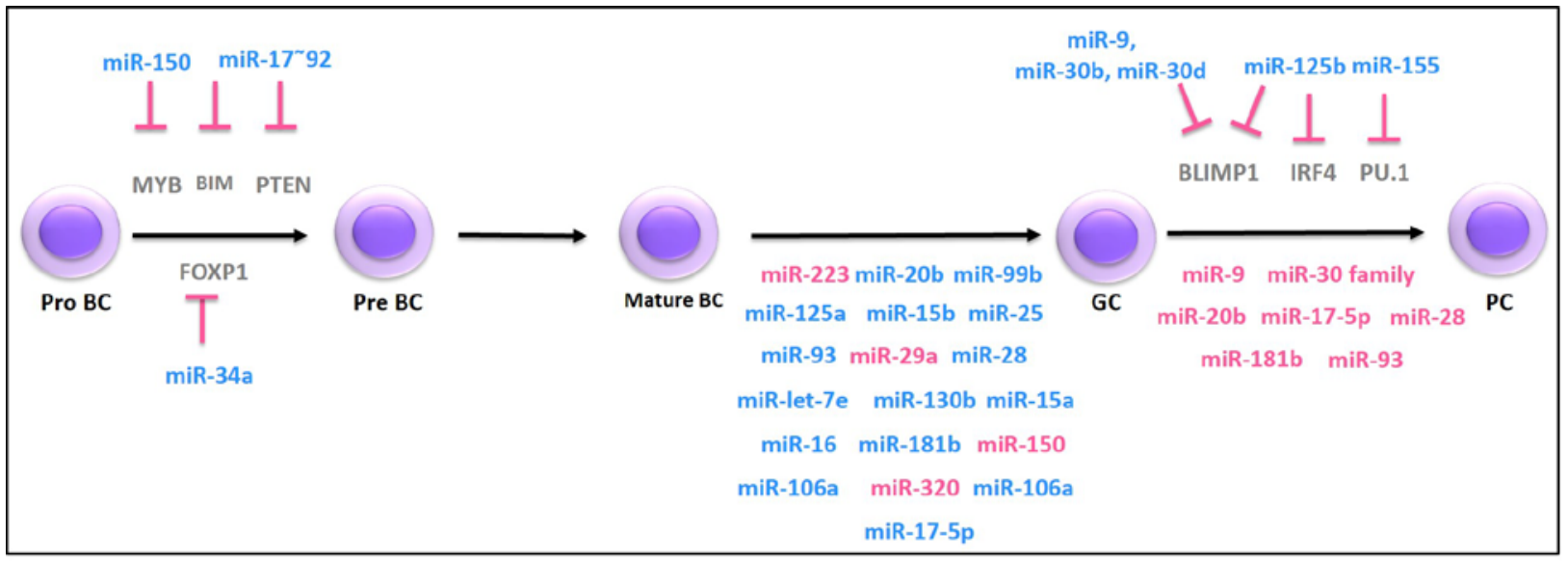

Figure 13. Involvement of microRNAS in B cell differentiation. miRNAs marked in blue are upregulated in the indicated transition, whereas those showed in red are downregulated. Adapted from Lionetti M et al ${ }^{231}$. 
References 

1. Hideshima T, Mitsiades C, Tonon G, Richardson PG, Anderson KC. Understanding multiple myeloma pathogenesis in the bone marrow to identify new therapeutic targets. Nat Rev Cancer. 2007;7(8):585-598.

2. Rajkumar SV and Kumar S. Multiple Myeloma: Diagnosis and Treatment. Mayo Clin Proc. 2016;91(1):101-119.

3. Kumar SK, Rajkumar V, Kyle RA, et al. Multiple myeloma. Nat Rev Dis Primers. 2017;3:17046.

4. Mahindra A, Hideshima T, Anderson KC. Multiple myeloma: biology of the disease. Blood Rev. 2010;24 Suppl 1:S5-11.

5. Raab MS, Podar K, Breitkreutz I, Richardson PG, Anderson KC. Multiple myeloma. Lancet. 2009;374(9686):324-339.

6. Mitsiades CS, Mitsiades N, Munshi NC, Anderson KC. Focus on multiple myeloma. Cancer Cell. 2004;6(5):439-444.

7. Morgan GJ, Walker BA, Davies FE. The genetic architecture of multiple myeloma. Nat Rev Cancer. 2012;12(5):335-348.

8. Kuehl WM and Bergsagel PL. Molecular pathogenesis of multiple myeloma and its premalignant precursor. J Clin Invest. 2012;122(10):3456-3463.

9. Kyle RA, Therneau TM, Rajkumar SV, et al. Prevalence of monoclonal gammopathy of undetermined significance. N Engl J Med. 2006;354(13):1362-1369.

10. Kyle RA and Rajkumar SV. Monoclonal gammopathy of undetermined significance. $\mathrm{Br} \mathrm{J}$ Haematol. 2006;134(6):573-589.

11. Rajkumar SV, Kyle RA, Therneau TM, et al. Serum free light chain ratio is an independent risk factor for progression in monoclonal gammopathy of undetermined significance. Blood. 2005;106(3):812-817.

12. Rajkumar SV. MGUS and smoldering multiple myeloma: update on pathogenesis, natural history, and management. Hematology Am Soc Hematol Educ Program. 2005:340-345.

13. Rajkumar SV, Landgren O, Mateos MV. Smoldering multiple myeloma. Blood. 2015;125(20):3069-3075.

14. Albarracin F and Fonseca R. Plasma cell leukemia. Blood Rev. 2011;25(3):107-112.

15. Kyle RA and Rajkumar SV. Criteria for diagnosis, staging, risk stratification and response assessment of multiple myeloma. Leukemia. 2009;23(1):3-9.

16. Rajkumar SV, Dimopoulos MA, Palumbo A, et al. International Myeloma Working Group updated criteria for the diagnosis of multiple myeloma. Lancet Oncol. 2014;15(12):e53848.

17. Kuehl WM and Bergsagel PL. Multiple myeloma: evolving genetic events and host interactions. Nat Rev Cancer. 2002;2(3):175-187. 
18. Kyle RA and Rajkumar SV. Multiple myeloma. N Engl J Med. 2004;351(18):1860-1873.

19. Fonseca R, Bergsagel PL, Drach J, et al. International Myeloma Working Group molecular classification of multiple myeloma: spotlight review. Leukemia. 2009;23(12):2210-2221.

20. Mikhael JR, Dingli D, Roy V, et al. Management of newly diagnosed symptomatic multiple myeloma: updated Mayo Stratification of Myeloma and Risk-Adapted Therapy (mSMART) consensus guidelines 2013. Mayo Clin Proc. 2013;88(4):360-376.

21. Dib A, Gabrea A, Glebov OK, Bergsagel PL, Kuehl WM. Characterization of MYC translocations in multiple myeloma cell lines. J Natl Cancer Inst Monogr. 2008;(39):25-31. doi(39):25-31.

22. Affer M, Chesi M, Chen WG, et al. Promiscuous MYC locus rearrangements hijack enhancers but mostly super-enhancers to dysregulate MYC expression in multiple myeloma. Leukemia. 2014;28(8):1725-1735.

23. Lopez-Corral L, Sarasquete ME, Bea S, et al. SNP-based mapping arrays reveal high genomic complexity in monoclonal gammopathies, from MGUS to myeloma status. Leukemia. 2012;26(12):2521-2529.

24. Walker BA, Mavrommatis K, Wardell CP, et al. A high-risk, Double-Hit, group of newly diagnosed myeloma identified by genomic analysis. Leukemia. 2019;33(1):159-170.

25. Avet-Loiseau H, Durie BG, Cavo M, et al. Combining fluorescent in situ hybridization data with ISS staging improves risk assessment in myeloma: an International Myeloma Working Group collaborative project. Leukemia. 2013;27(3):711-717.

26. Chapman MA, Lawrence MS, Keats JJ, et al. Initial genome sequencing and analysis of multiple myeloma. Nature. 2011;471(7339):467-472.

27. Lohr JG, Stojanov P, Carter SL, et al. Widespread genetic heterogeneity in multiple myeloma: implications for targeted therapy. Cancer Cell. 2014;25(1):91-101.

28. Walker BA, Wardell CP, Melchor L, et al. Intraclonal heterogeneity and distinct molecular mechanisms characterize the development of $\mathrm{t}(4 ; 14)$ and $\mathrm{t}(11 ; 14)$ myeloma. Blood. 2012;120(5):1077-1086.

29. Bolli N, Avet-Loiseau H, Wedge DC, et al. Heterogeneity of genomic evolution and mutational profiles in multiple myeloma. Nat Commun. 2014;5:2997.

30. Yellapantula V, Hultcrantz M, Rustad EH, et al. Comprehensive detection of recurring genomic abnormalities: a targeted sequencing approach for multiple myeloma. Blood Cancer J. 2019;9(12):101-019-0264-y.

31. Bolli N, Biancon G, Moarii M, et al. Analysis of the genomic landscape of multiple myeloma highlights novel prognostic markers and disease subgroups. Leukemia. 2018;32(12):2604-2616.

32. Bolli N, Biancon G, Moarii M, et al. Analysis of the genomic landscape of multiple myeloma highlights novel prognostic markers and disease subgroups. Leukemia. 2018;32(12):2604-2616. 
33. Hu Y, Chen W, Wang J. Progress in the identification of gene mutations involved in multiple myeloma. Onco Targets Ther. 2019;12:4075-4080.

34. Weissbach S, Langer C, Puppe B, et al. The molecular spectrum and clinical impact of DIS3 mutations in multiple myeloma. Br J Haematol. 2015;169(1):57-70.

35. Herrero AB, Quwaider D, Corchete LA, Mateos MV, Garcia-Sanz R, Gutierrez NC. FAM46C controls antibody production by the polyadenylation of immunoglobulin mRNAs and inhibits cell migration in multiple myeloma. J Cell Mol Med. 2020.

36. Manier S, Salem KZ, Park J, Landau DA, Getz G, Ghobrial IM. Genomic complexity of multiple myeloma and its clinical implications. Nat Rev Clin Oncol. 2017;14(2):100-113.

37. Mercier FE, Ragu C, Scadden DT. The bone marrow at the crossroads of blood and immunity. Nat Rev Immunol. 2011;12(1):49-60.

38. Kondo M. Lymphoid and myeloid lineage commitment in multipotent hematopoietic progenitors. Immunol Rev. 2010;238(1):37-46.

39. Mikkola HK and Orkin SH. The journey of developing hematopoietic stem cells. Development. 2006;133(19):3733-3744.

40. Weissman IL. Translating stem and progenitor cell biology to the clinic: barriers and opportunities. Science. 2000;287(5457):1442-1446.

41. Akashi K, Traver D, Miyamoto T, Weissman IL. A clonogenic common myeloid progenitor that gives rise to all myeloid lineages. Nature. 2000;404(6774):193-197.

42. Akashi K, Reya T, Dalma-Weiszhausz D, Weissman IL. Lymphoid precursors. Curr Opin Immunol. 2000;12(2):144-150.

43. Ambreen Shaikh and Deepa Bhartiya. DOI: 10.5772/48133.

44. Ribatti D, Crivellato E, Vacca A. The contribution of Bruce Glick to the definition of the role played by the bursa of Fabricius in the development of the B cell lineage. Clin Exp Immunol. 2006;145(1):1-4.

45. WARNER NL and SZENBERG A. The Immunological Function of the Bursa of Fabricius in the Chicken. Annu Rev Microbiol. 1964;18:253-268.

46. Roitt IM, Greaves MF, Torrigiani G, Brostoff J, Playfair JH. The cellular basis of immunological responses. A synthesis of some current views. Lancet. 1969;2(7616):367371.

47. Hibi T and Dosch HM. Limiting dilution analysis of the B cell compartment in human bone marrow. Eur J Immunol. 1986;16(2):139-145.

48. Oliver Backhaus. Generation of Antibody Diversity. DOI: 10.5772/intechopen.72818; 2018: 
49. Harris LJ, Larson SB, Hasel KW, Day J, Greenwood A, McPherson A. The threedimensional structure of an intact monoclonal antibody for canine lymphoma. Nature. 1992;360(6402):369-372.

50. Navia MA, Segal DM, Padlan EA, et al. Crystal structure of galactan-binding mouse immunoglobulin J539 Fab at 4.5-A resolution. Proc Natl Acad Sci U S A. 1979;76(8):40714074.

51. Titani K, Whitley E,Jr, Avogardo L, Putnam FW. Immunoglobulin structure: partial amino acid sequence of a Bence Jones protein. Science. 1965;149(3688):1090-1092.

52. Conley ME. Genes required for B cell development. J Clin Invest. 2003;112(11):16361638.

53. Rajewsky K. Clonal selection and learning in the antibody system. Nature. 1996;381(6585):751-758.

54. Kanyavuz A, Marey-Jarossay A, Lacroix-Desmazes S, Dimitrov JD. Breaking the law: unconventional strategies for antibody diversification. Nat Rev Immunol. 2019.

55. Kondo M. Lymphoid and myeloid lineage commitment in multipotent hematopoietic progenitors. Immunol Rev. 2010;238(1):37-46.

56. Hardy RR and Hayakawa K. B cell development pathways. Annu Rev Immunol. 2001;19:595-621.

57. Oettinger MA, Schatz DG, Gorka C, Baltimore D. RAG-1 and RAG-2, adjacent genes that synergistically activate V(D)J recombination. Science. 1990;248(4962):1517-1523.

58. Schatz DG and Baltimore D. Stable expression of immunoglobulin gene V(D)J recombinase activity by gene transfer into 3T3 fibroblasts. Cell. 1988;53(1):107-115.

59. Bashford-Rogers RJM, Smith KGC, Thomas DC. Antibody repertoire analysis in polygenic autoimmune diseases. Immunology. 2018;155(1):3-17.

60. Cooper MD. The early history of B cells. Nat Rev Immunol. 2015;15(3):191-197.

61. MacLennan IC. Germinal centers. Annu Rev Immunol. 1994;12:117-139.

62. LeBien TW and Tedder TF. B lymphocytes: how they develop and function. Blood. 2008;112(5):1570-1580.

63. Pelanda R and Torres RM. Central B-cell tolerance: where selection begins. Cold Spring Harb Perspect Biol. 2012;4(4):a007146.

64. Nieuwenhuis P and Ford WL. Comparative migration of B- and T-Lymphocytes in the rat spleen and lymph nodes. Cell Immunol. 1976;23(2):254-267.

65. Liu YJ, de Bouteiller O, Fugier-Vivier I. Mechanisms of selection and differentiation in germinal centers. Curr Opin Immunol. 1997;9(2):256-262. 
66. Honjo T, Kinoshita K, Muramatsu M. Molecular mechanism of class switch recombination: linkage with somatic hypermutation. Annu Rev Immunol. 2002;20:165-196.

67. Klein U and Dalla-Favera R. Germinal centres: role in B-cell physiology and malignancy. Nat Rev Immunol. 2008;8(1):22-33.

68. Berek C, Berger A, Apel M. Maturation of the immune response in germinal centers. Cell. 1991;67(6):1121-1129.

69. Kuppers R, Zhao M, Hansmann ML, Rajewsky K. Tracing B cell development in human germinal centres by molecular analysis of single cells picked from histological sections. EMBO J. 1993;12(13):4955-4967.

70. Jacob J, Kelsoe G, Rajewsky K, Weiss U. Intraclonal generation of antibody mutants in germinal centres. Nature. 1991;354(6352):389-392.

71. Di Noia JM and Neuberger MS. Molecular mechanisms of antibody somatic hypermutation. Annu Rev Biochem. 2007;76:1-22.

72. De Silva NS and Klein U. Dynamics of B cells in germinal centres. Nat Rev Immunol. 2015;15(3):137-148.

73. Liu YJ, Zhang J, Lane PJ, Chan EY, MacLennan IC. Sites of specific B cell activation in primary and secondary responses to $\mathrm{T}$ cell-dependent and $\mathrm{T}$ cell-independent antigens. Eur J Immunol. 1991;21(12):2951-2962.

74. Kirk SJ, Cliff JM, Thomas JA, Ward TH. Biogenesis of secretory organelles during B cell differentiation. J Leukoc Biol. 2010;87(2):245-255.

75. King LB and Corley RB. Characterization of a presecretory phase in B-cell differentiation. Proc Natl Acad Sci U S A. 1989;86(8):2814-2818.

76. Gass JN, Gunn KE, Sriburi R, Brewer JW. Stressed-out B cells? Plasma-cell differentiation and the unfolded protein response. Trends Immunol. 2004;25(1):17-24.

77. Barneda-Zahonero B, Roman-Gonzalez L, Collazo O, Mahmoudi T, Parra M. Epigenetic regulation of B lymphocyte differentiation, transdifferentiation, and reprogramming. Comp Funct Genomics. 2012;2012:564381.

78. Limon JJ and Fruman DA. Akt and mTOR in B Cell Activation and Differentiation. Front Immunol. 2012;3:228.

79. Nutt SL, Taubenheim N, Hasbold J, Corcoran LM, Hodgkin PD. The genetic network controlling plasma cell differentiation. Semin Immunol. 2011;23(5):341-349.

80. Calame KL, Lin KI, Tunyaplin C. Regulatory mechanisms that determine the development and function of plasma cells. Annu Rev Immunol. 2003;21:205-230.

81. Shapiro-Shelef $M$ and Calame K. Regulation of plasma-cell development. Nat Rev Immunol. 2005;5(3):230-242. 
82. O'Riordan M and Grosschedl R. Coordinate regulation of B cell differentiation by the transcription factors EBF and E2A. Immunity. 1999;11(1):21-31.

83. Nutt SL, Hodgkin PD, Tarlinton DM, Corcoran LM. The generation of antibody-secreting plasma cells. Nat Rev Immunol. 2015;15(3):160-171.

84. Cobaleda C, Schebesta A, Delogu A, Busslinger M. Pax5: the guardian of B cell identity and function. Nat Immunol. 2007;8(5):463-470.

85. Nutt SL, Heavey B, Rolink AG, Busslinger M. Commitment to the B-lymphoid lineage depends on the transcription factor Pax5. Nature. 1999;401(6753):556-562.

86. Revilla-I-Domingo R, Bilic I, Vilagos B, et al. The B-cell identity factor Pax5 regulates distinct transcriptional programmes in early and late $\mathrm{B}$ lymphopoiesis. EMBO J. 2012;31(14):3130-3146.

87. Cobaleda C, Jochum W, Busslinger M. Conversion of mature B cells into $\mathrm{T}$ cells by dedifferentiation to uncommitted progenitors. Nature. 2007;449(7161):473-477.

88. Calame KL, Lin KI, Tunyaplin C. Regulatory mechanisms that determine the development and function of plasma cells. Annu Rev Immunol. 2003;21:205-230.

89. Pridans C, Holmes ML, Polli M, et al. Identification of Pax5 target genes in early B cell differentiation. J Immunol. 2008;180(3):1719-1728.

90. Schebesta A, McManus S, Salvagiotto G, Delogu A, Busslinger GA, Busslinger M. Transcription factor Pax5 activates the chromatin of key genes involved in B cell signaling, adhesion, migration, and immune function. Immunity. 2007;27(1):49-63.

91. Horcher M, Souabni A, Busslinger M. Pax5/BSAP maintains the identity of B cells in late B lymphopoiesis. Immunity. 2001;14(6):779-790.

92. Reimold AM, Ponath PD, Li YS, et al. Transcription factor B cell lineage-specific activator protein regulates the gene for human X-box binding protein 1. J Exp Med. 1996;183(2):393401.

93. Cattoretti G, Chang CC, Cechova K, et al. BCL- 6 protein is expressed in germinal-center B cells. Blood. 1995;86(1):45-53.

94. Angelin-Duclos C, Cattoretti G, Lin KI, Calame K. Commitment of B lymphocytes to a plasma cell fate is associated with Blimp-1 expression in vivo. J Immunol. 2000;165(10):5462-5471.

95. Dent AL, Shaffer AL, Yu X, Allman D, Staudt LM. Control of inflammation, cytokine expression, and germinal center formation by BCL-6. Science. 1997;276(5312):589-592.

96. Shaffer AL, Yu X, He Y, Boldrick J, Chan EP, Staudt LM. BCL-6 represses genes that function in lymphocyte differentiation, inflammation, and cell cycle control. Immunity. 2000;13(2):199-212.

97. Tunyaplin C, Shaffer AL, Angelin-Duclos CD, Yu X, Staudt LM, Calame KL. Direct repression of prdm 1 by Bcl-6 inhibits plasmacytic differentiation. J Immunol. 2004;173(2):1158-1165. 
98. Fearon DT, Manders PM, Wagner SD. Bcl-6 uncouples B lymphocyte proliferation from differentiation. Adv Exp Med Biol. 2002;512:21-28.

99. Cimmino L, Martins GA, Liao J, et al. Blimp-1 attenuates Th1 differentiation by repression of ifng, tbx21, and bcl6 gene expression. J Immunol. 2008;181(4):2338-2347.

100. Saito M, Gao J, Basso K, et al. A signaling pathway mediating downregulation of BCL6 in germinal center B cells is blocked by BCL6 gene alterations in B cell lymphoma. Cancer Cell. 2007;12(3):280-292.

101. Fujita N, Jaye DL, Geigerman C, et al. MTA3 and the Mi-2/NuRD complex regulate cell fate during B lymphocyte differentiation. Cell. 2004;119(1):75-86.

102. Lin L, Gerth AJ, Peng SL. Active inhibition of plasma cell development in resting B cells by microphthalmia-associated transcription factor. J Exp Med. 2004;200(1):115-122.

103. Muto A, Tashiro S, Nakajima 0 , et al. The transcriptional programme of antibody class switching involves the repressor Bach2. Nature. 2004;429(6991):566-571.

104. Ochiai K, Katoh Y, Ikura T, et al. Plasmacytic transcription factor Blimp-1 is repressed by Bach2 in B cells. J Biol Chem. 2006;281(50):38226-38234.

105. Ochiai K, Muto A, Tanaka H, Takahashi S, Igarashi K. Regulation of the plasma cell transcription factor Blimp-1 gene by Bach2 and Bcl6. Int Immunol. 2008;20(3):453-460.

106. Kallies A, Hasbold J, Fairfax K, et al. Initiation of plasma-cell differentiation is independent of the transcription factor Blimp-1. Immunity. 2007;26(5):555-566.

107. Shapiro-Shelef M, Lin KI, McHeyzer-Williams LJ, Liao J, McHeyzer-Williams MG, Calame $\mathrm{K}$. Blimp-1 is required for the formation of immunoglobulin secreting plasma cells and preplasma memory B cells. Immunity. 2003;19(4):607-620.

108. Lin KI, Angelin-Duclos C, Kuo TC, Calame K. Blimp-1-dependent repression of Pax-5 is required for differentiation of B cells to immunoglobulin M-secreting plasma cells. Mol Cell Biol. 2002;22(13):4771-4780.

109. Shaffer AL, Lin KI, Kuo TC, et al. Blimp-1 orchestrates plasma cell differentiation by extinguishing the mature B cell gene expression program. Immunity. 2002;17(1):51-62.

110. Kallies A, Hasbold J, Tarlinton DM, et al. Plasma cell ontogeny defined by quantitative changes in blimp-1 expression. J Exp Med. 2004;200(8):967-977.

111. Sciammas R and Davis MM. Modular nature of Blimp-1 in the regulation of gene expression during B cell maturation. J Immunol. 2004;172(9):5427-5440.

112. Shaffer AL, Shapiro-Shelef M, Iwakoshi NN, et al. XBP1, downstream of Blimp-1, expands the secretory apparatus and other organelles, and increases protein synthesis in plasma cell differentiation. Immunity. 2004;21(1):81-93.

113. Tellier J, Shi W, Minnich M, et al. Blimp-1 controls plasma cell function through the regulation of immunoglobulin secretion and the unfolded protein response. Nat Immunol. 2016;17(3):323-330. 
114. Mora-Lopez F, Reales E, Brieva JA, Campos-Caro A. Human BSAP and BLIMP1 conform an autoregulatory feedback loop. Blood. 2007;110(9):3150-3157.

115. Lin L, Gerth AJ, Peng SL. Active inhibition of plasma cell development in resting B cells by microphthalmia-associated transcription factor. J Exp Med. 2004;200(1):115-122.

116. Sciammas R, Shaffer AL, Schatz JH, Zhao H, Staudt LM, Singh H. Graded expression of interferon regulatory factor-4 coordinates isotype switching with plasma cell differentiation. Immunity. 2006;25(2):225-236.

117. Klein U, Casola S, Cattoretti G, et al. Transcription factor IRF4 controls plasma cell differentiation and class-switch recombination. Nat Immunol. 2006;7(7):773-782.

118. Ochiai K, Maienschein-Cline M, Simonetti G, et al. Transcriptional regulation of germinal center $\mathrm{B}$ and plasma cell fates by dynamical control of IRF4. Immunity. 2013;38(5):918-929.

119. Willis SN, Good-Jacobson KL, Curtis J, et al. Transcription factor IRF4 regulates germinal center cell formation through a B cell-intrinsic mechanism. J Immunol. 2014;192(7):3200-3206.

120. Chevrier S, Emslie D, Shi W, et al. The BTB-ZF transcription factor Zbtb20 is driven by Irf4 to promote plasma cell differentiation and longevity. J Exp Med. 2014;211(5):827-840.

121. Sciammas R, Li Y, Warmflash A, Song Y, Dinner AR, Singh H. An incoherent regulatory network architecture that orchestrates $B$ cell diversification in response to antigen signaling. Mol Syst Biol. 2011;7:495.

122. Reimold AM, Iwakoshi NN, Manis J, et al. Plasma cell differentiation requires the transcription factor XBP-1. Nature. 2001;412(6844):300-307.

123. Yoshida H, Matsui T, Yamamoto A, Okada T, Mori K. XBP1 mRNA is induced by ATF6 and spliced by IRE1 in response to ER stress to produce a highly active transcription factor. Cell. 2001;107(7):881-891.

124. Todd DJ, Lee AH, Glimcher LH. The endoplasmic reticulum stress response in immunity and autoimmunity. Nat Rev Immunol. 2008;8(9):663-674.

125. Leung-Hagesteijn C, Erdmann N, Cheung G, et al. Xbp1s-negative tumor B cells and preplasmablasts mediate therapeutic proteasome inhibitor resistance in multiple myeloma. Cancer Cell. 2013;24(3):289-304.

126. Shaffer AL, Emre NC, Lamy L, et al. IRF4 addiction in multiple myeloma. Nature. 2008;454(7201):226-231.

127. Peperzak V, Vikstrom I, Walker J, et al. Mcl-1 is essential for the survival of plasma cells. Nat Immunol. 2013;14(3):290-297.

128. Igarashi $\mathrm{K}$, Ochiai $\mathrm{K}$, Muto $\mathrm{A}$. Architecture and dynamics of the transcription factor network that regulates B-to-plasma cell differentiation. J Biochem. 2007;141(6):783-789. 
129. Wiest DL, Burkhardt JK, Hester S, Hortsch M, Meyer DI, Argon Y. Membrane biogenesis during B cell differentiation: most endoplasmic reticulum proteins are expressed coordinately. J Cell Biol. 1990;110(5):1501-1511.

130. Gass JN, Gifford NM, Brewer JW. Activation of an unfolded protein response during differentiation of antibody-secreting B cells. J Biol Chem. 2002;277(50):49047-49054.

131. Grootjans J, Kaser A, Kaufman RJ, Blumberg RS. The unfolded protein response in immunity and inflammation. Nat Rev Immunol. 2016;16(8):469-484.

132. Bertolotti A, Zhang Y, Hendershot LM, Harding HP, Ron D. Dynamic interaction of BiP and ER stress transducers in the unfolded-protein response. Nat Cell Biol. 2000;2(6):326332.

133. Shen J, Chen X, Hendershot L, Prywes R. ER stress regulation of ATF6 localization by dissociation of BiP/GRP78 binding and unmasking of Golgi localization signals. Dev Cell. 2002;3(1):99-111.

134. Lee AS. The ER chaperone and signaling regulator GRP78/BiP as a monitor of endoplasmic reticulum stress. Methods. 2005;35(4):373-381.

135. Vincenz L, Jager R, O'Dwyer M, Samali A. Endoplasmic reticulum stress and the unfolded protein response: targeting the Achilles heel of multiple myeloma. Mol Cancer Ther. 2013;12(6):831-843.

136. Shi Y, Vattem KM, Sood R, et al. Identification and characterization of pancreatic eukaryotic initiation factor 2 alpha-subunit kinase, PEK, involved in translational control. Mol Cell Biol. 1998;18(12):7499-7509.

137. Harding HP, Zhang Y, Ron D. Protein translation and folding are coupled by an endoplasmic-reticulum-resident kinase. Nature. 1999;397(6716):271-274.

138. McQuiston A and Diehl JA. Recent insights into PERK-dependent signaling from the stressed endoplasmic reticulum. F1000Res. 2017;6:1897.

139. Rutkowski DT and Kaufman RJ. A trip to the ER: coping with stress. Trends Cell Biol. 2004;14(1):20-28.

140. Harding HP, Zhang Y, Ron D. Protein translation and folding are coupled by an endoplasmic-reticulum-resident kinase. Nature. 1999;397(6716):271-274.

141. Harding HP, Zhang Y, Ron D. Protein translation and folding are coupled by an endoplasmic-reticulum-resident kinase. Nature. 1999;397(6716):271-274.

142. Harding HP, Zhang Y, Bertolotti A, Zeng H, Ron D. Perk is essential for translational regulation and cell survival during the unfolded protein response. Mol Cell. 2000;5(5):897904.

143. Harding HP, Zhang $\mathrm{Y}$, Zeng $\mathrm{H}$, et al. An integrated stress response regulates amino acid metabolism and resistance to oxidative stress. Mol Cell. 2003;11(3):619-633. 
144. Yoshida H, Okada T, Haze K, et al. ATF6 activated by proteolysis binds in the presence of NF-Y (CBF) directly to the cis-acting element responsible for the mammalian unfolded protein response. Mol Cell Biol. 2000;20(18):6755-6767.

145. Li M, Baumeister P, Roy B, et al. ATF6 as a transcription activator of the endoplasmic reticulum stress element: thapsigargin stress-induced changes and synergistic interactions with NF-Y and YY1. Mol Cell Biol. 2000;20(14):5096-5106.

146. Ye J, Rawson RB, Komuro R, et al. ER stress induces cleavage of membrane-bound ATF6 by the same proteases that process SREBPs. Mol Cell. 2000;6(6):1355-1364.

147. Yoshida H, Okada T, Haze K, et al. ATF6 activated by proteolysis binds in the presence of NF-Y (CBF) directly to the cis-acting element responsible for the mammalian unfolded protein response. Mol Cell Biol. 2000;20(18):6755-6767.

148. Haze K, Yoshida H, Yanagi H, Yura T, Mori K. Mammalian transcription factor ATF6 is synthesized as a transmembrane protein and activated by proteolysis in response to endoplasmic reticulum stress. Mol Biol Cell. 1999;10(11):3787-3799.

149. Prischi F, Nowak PR, Carrara M, Ali MM. Phosphoregulation of Ire1 RNase splicing activity. Nat Commun. 2014;5:3554.

150. Oikawa D, Kimata Y, Kohno K, Iwawaki T. Activation of mammalian IRE1alpha upon ER stress depends on dissociation of BiP rather than on direct interaction with unfolded proteins. Exp Cell Res. 2009;315(15):2496-2504.

151. Promlek T, Ishiwata-Kimata Y, Shido M, Sakuramoto M, Kohno K, Kimata Y. Membrane aberrancy and unfolded proteins activate the endoplasmic reticulum stress sensor Ire1 in different ways. Mol Biol Cell. 2011;22(18):3520-3532.

152. Yoshida H, Matsui T, Yamamoto A, Okada T, Mori K. XBP1 mRNA is induced by ATF6 and spliced by IRE1 in response to ER stress to produce a highly active transcription factor. Cell. 2001;107(7):881-891.

153. Calfon $\mathrm{M}$, Zeng $\mathrm{H}$, Urano $\mathrm{F}$, et al. IRE1 couples endoplasmic reticulum load to secretory capacity by processing the XBP-1 mRNA. Nature. 2002;415(6867):92-96.

154. Lee AH, Iwakoshi NN, Glimcher LH. XBP-1 regulates a subset of endoplasmic reticulum resident chaperone genes in the unfolded protein response. Mol Cell Biol. 2003;23(21):7448-7459.

155. Meusser B, Hirsch C, Jarosch E, Sommer T. ERAD: the long road to destruction. Nat Cell Biol. 2005;7(8):766-772.

156. Wu J and Kaufman RJ. From acute ER stress to physiological roles of the Unfolded Protein Response. Cell Death Differ. 2006;13(3):374-384.

157. Payne KJ and Crooks GM. Human hematopoietic lineage commitment. Immunol Rev. 2002;187:48-64.

158. Wood B. Multicolor immunophenotyping: human immune system hematopoiesis. Methods Cell Biol. 2004;75:559-576. 
159. Griffin DO, Holodick NE, Rothstein TL. Human B1 cells in umbilical cord and adult peripheral blood express the novel phenotype CD20+ CD27+ CD43+ CD70-. J Exp Med. 2011;208(1):67-80.

160. Manjarrez-Orduno N, Quach TD, Sanz I. B cells and immunological tolerance. J Invest Dermatol. 2009;129(2):278-288.

161. Haas KM and Tedder TF. Role of the CD19 and CD21/35 receptor complex in innate immunity, host defense and autoimmunity. Adv Exp Med Biol. 2005;560:125-139.

162. Wu H, Deng Y, Feng $\mathrm{Y}$, et al. Epigenetic regulation in B-cell maturation and its dysregulation in autoimmunity. Cell Mol Immunol. 2018;15(7):676-684.

163. Barneda-Zahonero B, Roman-Gonzalez L, Collazo O, Mahmoudi T, Parra M. Epigenetic regulation of $\mathrm{B}$ lymphocyte differentiation, transdifferentiation, and reprogramming. Comp Funct Genomics. 2012;2012:564381.

164. Zan H and Casali P. Epigenetics of Peripheral B-Cell Differentiation and the Antibody Response. Front Immunol. 2015;6:631.

165. Shaknovich R, Cerchietti L, Tsikitas L, et al. DNA methyltransferase 1 and DNA methylation patterning contribute to germinal center B-cell differentiation. Blood. 2011;118(13):3559-3569.

166. Cobaleda C, Schebesta A, Delogu A, Busslinger M. Pax5: the guardian of B cell identity and function. Nat Immunol. 2007;8(5):463-470.

167. Yu J, Angelin-Duclos C, Greenwood J, Liao J, Calame K. Transcriptional repression by blimp-1 (PRDI-BF1) involves recruitment of histone deacetylase. Mol Cell Biol. 2000;20(7):2592-2603.

168. Gyory I, Wu J, Fejer G, Seto E, Wright KL. PRDI-BF1 recruits the histone H3 methyltransferase G9a in transcriptional silencing. Nat Immunol. 2004;5(3):299-308.

169. Li G, Zan H, Xu Z, Casali P. Epigenetics of the antibody response. Trends Immunol. 2013;34(9):460-470.

170. Esteller M. Non-coding RNAs in human disease. Nat Rev Genet. 2011;12(12):861-874.

171. Lee RC, Feinbaum RL, Ambros V. The C. elegans heterochronic gene lin-4 encodes small RNAs with antisense complementarity to lin-14. Cell. 1993;75(5):843-854.

172. Bartel DP. MicroRNAs: genomics, biogenesis, mechanism, and function. Cell. 2004;116(2):281-297.

173. Alles J, Fehlmann T, Fischer U, et al. An estimate of the total number of true human miRNAs. Nucleic Acids Res. 2019;47(7):3353-3364.

174. Ambros V. The functions of animal microRNAs. Nature. 2004;431(7006):350-355.

175. Benetatos L and Vartholomatos G. Deregulated microRNAs in multiple myeloma. Cancer. 2012;118(4):878-887. 
176. Davis-Dusenbery BN and Hata A. Mechanisms of control of microRNA biogenesis. J Biochem. 2010;148(4):381-392.

177. Winter J, Jung S, Keller S, Gregory RI, Diederichs S. Many roads to maturity: microRNA biogenesis pathways and their regulation. Nat Cell Biol. 2009;11(3):228-234.

178. Bartel DP. MicroRNAs: target recognition and regulatory functions. Cell. 2009;136(2):215-233.

179. Shukla GC, Singh J, Barik S. MicroRNAs: Processing, Maturation, Target Recognition and Regulatory Functions. Mol Cell Pharmacol. 2011;3(3):83-92.

180. Marques SC, Laursen MB, Bodker JS, et al. MicroRNAs in B-cells: from normal differentiation to treatment of malignancies. Oncotarget. 2015;6(1):7-25.

181. Baskerville S and Bartel DP. Microarray profiling of microRNAs reveals frequent coexpression with neighboring miRNAs and host genes. RNA. 2005;11(3):241-247.

182. Altuvia $Y$, Landgraf $P$, Lithwick $G$, et al. Clustering and conservation patterns of human microRNAs. Nucleic Acids Res. 2005;33(8):2697-2706.

183. Rupaimoole R, Calin GA, Lopez-Berestein G, Sood AK. miRNA Deregulation in Cancer Cells and the Tumor Microenvironment. Cancer Discov. 2016;6(3):235-246.

184. Borchert GM, Lanier W, Davidson BL. RNA polymerase III transcribes human microRNAs. Nat Struct Mol Biol. 2006;13(12):1097-1101.

185. Lee Y, Ahn C, Han J, et al. The nuclear RNase III Drosha initiates microRNA processing. Nature. 2003;425(6956):415-419.

186. Han J, Lee Y, Yeom KH, et al. Molecular basis for the recognition of primary microRNAs by the Drosha-DGCR8 complex. Cell. 2006;125(5):887-901.

187. Zeng Y and Cullen BR. Structural requirements for pre-microRNA binding and nuclear export by Exportin 5. Nucleic Acids Res. 2004;32(16):4776-4785.

188. Winter J, Jung S, Keller S, Gregory RI, Diederichs S. Many roads to maturity: microRNA biogenesis pathways and their regulation. Nat Cell Biol. 2009;11(3):228-234.

189. Ha M and Kim VN. Regulation of microRNA biogenesis. Nat Rev Mol Cell Biol. 2014;15(8):509-524.

190. Biasiolo M, Sales G, Lionetti M, et al. Impact of host genes and strand selection on miRNA and miRNA* expression. PLoS One. 2011;6(8):e23854.

191. Kuchenbauer F, Mah SM, Heuser M, et al. Comprehensive analysis of mammalian miRNA* species and their role in myeloid cells. Blood. 2011;118(12):3350-3358.

192. Ambros V. The functions of animal microRNAs. Nature. 2004;431(7006):350-355. 
193. Misiewicz-Krzeminska I, Krzeminski P, Corchete LA, et al. Factors Regulating microRNA Expression and Function in Multiple Myeloma. Noncoding RNA. 2019;5(1):10.3390/ncrna5010009.

194. Ambros V. MicroRNAs and developmental timing. Curr Opin Genet Dev. 2011;21(4):511-517.

195. Ambros $\mathrm{V}$ and Chen $\mathrm{X}$. The regulation of genes and genomes by small RNAs. Development. 2007;134(9):1635-1641.

196. Bartel DP. Metazoan MicroRNAs. Cell. 2018;173(1):20-51.

197. Zhang B, Pan X, Cobb GP, Anderson TA. microRNAs as oncogenes and tumor suppressors. Dev Biol. 2007;302(1):1-12.

198. Kasinski AL and Slack FJ. Epigenetics and genetics. MicroRNAs en route to the clinic: progress in validating and targeting microRNAs for cancer therapy. Nat Rev Cancer. 2011;11(12):849-864.

199. Pichiorri F, Suh SS, Ladetto M, et al. MicroRNAs regulate critical genes associated with multiple myeloma pathogenesis. Proc Natl Acad Sci U S A. 2008;105(35):12885-12890.

200. Lionetti M, Biasiolo M, Agnelli L, et al. Identification of microRNA expression patterns and definition of a microRNA/mRNA regulatory network in distinct molecular groups of multiple myeloma. Blood. 2009;114(25):e20-6.

201. Li Y, Zhang B, Li W, et al. MiR-15a/16 regulates the growth of myeloma cells, angiogenesis and antitumor immunity by inhibiting Bcl-2, VEGF-A and IL-17 expression in multiple myeloma. Leuk Res. 2016;49:73-79.

202. Amodio N, Stamato MA, Gulla AM, et al. Therapeutic Targeting of miR-29b/HDAC4 Epigenetic Loop in Multiple Myeloma. Mol Cancer Ther. 2016;15(6):1364-1375.

203. Lu Y, Wu D, Wang J, Li Y, Chai X, Kang Q. miR-320a regulates cell proliferation and apoptosis in multiple myeloma by targeting pre-B-cell leukemia transcription factor 3 . Biochem Biophys Res Commun. 2016;473(4):1315-1320.

204. Yu T, Zhang X, Zhang L, et al. MicroRNA-497 suppresses cell proliferation and induces apoptosis through targeting PBX3 in human multiple myeloma. Am J Cancer Res. 2016;6(12):2880-2889.

205. Yang Y, Li F, Saha MN, Abdi J, Qiu L, Chang H. miR-137 and miR-197 Induce Apoptosis and Suppress Tumorigenicity by Targeting MCL-1 in Multiple Myeloma. Clin Cancer Res. 2015;21(10):2399-2411.

206. Di Martino MT, Amodio N, Tassone P, Tagliaferri P. Functional Analysis of microRNA in Multiple Myeloma. Methods Mol Biol. 2016;1375:181-194.

207. Ahmad N, Haider S, Jagannathan S, Anaissie E, Driscoll JJ. MicroRNA theragnostics for the clinical management of multiple myeloma. Leukemia. 2014;28(4):732-738. 
208. Amodio N, Di Martino MT, Neri A, Tagliaferri P, Tassone P. Non-coding RNA: a novel opportunity for the personalized treatment of multiple myeloma. Expert Opin Biol Ther. 2013;13 Suppl 1:S125-37.

209. Tagliaferri P, Rossi M, Di Martino MT, et al. Promises and challenges of MicroRNAbased treatment of multiple myeloma. Curr Cancer Drug Targets. 2012;12(7):838-846.

210. Raimondi L, De Luca A, Morelli E, et al. MicroRNAs: Novel Crossroads between Myeloma Cells and the Bone Marrow Microenvironment. Biomed Res Int. 2016;2016:6504593.

211. Gutierrez NC, Sarasquete ME, Misiewicz-Krzeminska I, et al. Deregulation of microRNA expression in the different genetic subtypes of multiple myeloma and correlation with gene expression profiling. Leukemia. 2010;24(3):629-637.

212. Herrero AB, Rojas EA, Misiewicz-Krzeminska I, Krzeminski P, Gutierrez NC. Molecular Mechanisms of p53 Deregulation in Cancer: An Overview in Multiple Myeloma. Int J Mol Sci. 2016;17(12):10.3390/ijms17122003.

213. Kumar M, Lu Z, Takwi AA, et al. Negative regulation of the tumor suppressor p53 gene by microRNAs. Oncogene. 2011;30(7):843-853.

214. Leotta M, Biamonte L, Raimondi L, et al. A p53-dependent tumor suppressor network is induced by selective miR-125a-5p inhibition in multiple myeloma cells. J Cell Physiol. 2014;229(12):2106-2116.

215. Murray MY, Rushworth SA, Zaitseva L, Bowles KM, Macewan DJ. Attenuation of dexamethasone-induced cell death in multiple myeloma is mediated by miR-125b expression. Cell Cycle. 2013;12(13):2144-2153.

216. Manikandan M, Deva Magendhra Rao AK, Arunkumar G, Rajkumar KS, Rajaraman R, Munirajan AK. Down Regulation of miR-34a and miR-143 May Indirectly Inhibit p53 in Oral Squamous Cell Carcinoma: a Pilot Study. Asian Pac J Cancer Prev. 2015;16(17):7619-7625.

217. Liu J, Zhang C, Zhao Y, Feng Z. MicroRNA Control of p53. J Cell Biochem. 2017;118(1):714.

218. Yamakuchi M, Ferlito M, Lowenstein CJ. miR-34a repression of SIRT1 regulates apoptosis. Proc Natl Acad Sci U S A. 2008;105(36):13421-13426.

219. Murray MY, Rushworth SA, Zaitseva L, Bowles KM, Macewan DJ. Attenuation of dexamethasone-induced cell death in multiple myeloma is mediated by miR-125b expression. Cell Cycle. 2013;12(13):2144-2153.

220. Pichiorri F, Suh SS, Rocci A, et al. Downregulation of p53-inducible microRNAs 192, 194, and 215 impairs the p53/MDM2 autoregulatory loop in multiple myeloma development. Cancer Cell. 2010;18(4):367-381.

221. Misiewicz-Krzeminska I, Sarasquete ME, Quwaider D, et al. Restoration of microRNA214 expression reduces growth of myeloma cells through positive regulation of P53 and inhibition of DNA replication. Haematologica. 2013;98(4):640-648. 
222. Liu Z, Zhang G, Yu W, Gao N, Peng J. miR-186 inhibits cell proliferation in multiple myeloma by repressing Jagged1. Biochem Biophys Res Commun. 2016;469(3):692-697.

223. Roccaro AM, Sacco A, Thompson B, et al. MicroRNAs 15a and 16 regulate tumor proliferation in multiple myeloma. Blood. 2009;113(26):6669-6680.

224. Misso G, Di Martino MT, De Rosa G, et al. Mir-34: a new weapon against cancer? Mol Ther Nucleic Acids. 2014;3:e194.

225. Di Martino MT, Leone E, Amodio N, et al. Synthetic miR-34a mimics as a novel therapeutic agent for multiple myeloma: in vitro and in vivo evidence. Clin Cancer Res. 2012;18(22):6260-6270.

226. Krzeminski P, Sarasquete ME, Misiewicz-Krzeminska I, et al. Insights into epigenetic regulation of microRNA-155 expression in multiple myeloma. Biochim Biophys Acta. 2015;1849(3):353-366.

227. Zhou Y, Chen L, Barlogie B, et al. High-risk myeloma is associated with global elevation of miRNAs and overexpression of EIF2C2/AG02. Proc Natl Acad Sci U S A. 2010;107(17):7904-7909.

228. Xu Q, Hou YX, Langlais $P$, et al. Expression of the cereblon binding protein argonaute 2 plays an important role for multiple myeloma cell growth and survival. BMC Cancer. 2016;16:297-016-2331-0.

229. Sarasquete ME, Gutierrez NC, Misiewicz-Krzeminska I, et al. Upregulation of Dicer is more frequent in monoclonal gammopathies of undetermined significance than in multiple myeloma patients and is associated with longer survival in symptomatic myeloma patients. Haematologica. 2010;96(3):468-471.

230. de Yebenes VG, Bartolome-Izquierdo N, Ramiro AR. Regulation of B-cell development and function by microRNAs. Immunol Rev. 2013;253(1):25-39.

231. Lionetti M, Agnelli L, Lombardi L, Tassone P, Neri A. MicroRNAs in the pathobiology of multiple myeloma. Curr Cancer Drug Targets. 2012;12(7):823-837.

232. Li J, Wan Y, Ji Q, Fang Y, Wu Y. The role of microRNAs in B-cell development and function. Cell Mol Immunol. 2013;10(2):107-112.

233. Chen CZ, Li L, Lodish HF, Bartel DP. MicroRNAs modulate hematopoietic lineage differentiation. Science. 2004;303(5654):83-86.

234. Fragoso R, Mao T, Wang S, et al. Modulating the strength and threshold of NOTCH oncogenic signals by mir-181a-1/b-1. PLoS Genet. 2012;8(8):e1002855.

235. Li QJ, Chau J, Ebert PJ, et al. miR-181a is an intrinsic modulator of T cell sensitivity and selection. Cell. 2007;129(1):147-161.

236. Ebert PJ, Jiang S, Xie J, Li QJ, Davis MM. An endogenous positively selecting peptide enhances mature $\mathrm{T}$ cell responses and becomes an autoantigen in the absence of microRNA miR-181a. Nat Immunol. 2009;10(11):1162-1169. 
237. Thai TH, Calado DP, Casola $S$, et al. Regulation of the germinal center response by microRNA-155. Science. 2007;316(5824):604-608.

238. Rodriguez A, Vigorito E, Clare $\mathrm{S}$, et al. Requirement of bic/microRNA-155 for normal immune function. Science. 2007;316(5824):608-611.

239. Vigorito E, Perks KL, Abreu-Goodger C, et al. microRNA-155 regulates the generation of immunoglobulin class-switched plasma cells. Immunity. 2007;27(6):847-859.

240. Calame K. MicroRNA-155 function in B Cells. Immunity. 2007;27(6):825-827.

241. Malumbres R, Sarosiek KA, Cubedo E, et al. Differentiation stage-specific expression of microRNAs in B lymphocytes and diffuse large B-cell lymphomas. Blood. 2009;113(16):3754-3764.

242. White CA, Pone EJ, Lam T, et al. Histone deacetylase inhibitors upregulate B cell microRNAs that silence AID and Blimp-1 expression for epigenetic modulation of antibody and autoantibody responses. J Immunol. 2014;193(12):5933-5950.

243. Zhang J, Jima DD, Jacobs C, et al. Patterns of microRNA expression characterize stages of human B-cell differentiation. Blood. 2009;113(19):4586-4594.

244. Gururajan M, Haga CL, Das S, et al. MicroRNA 125b inhibition of B cell differentiation in germinal centers. Int Immunol. 2010;22(7):583-592.

245. Shen T, Sanchez HN, Zan H, Casali P. Genome-Wide Analysis Reveals Selective Modulation of microRNAs and mRNAs by Histone Deacetylase Inhibitor in B Cells Induced to Undergo Class-Switch DNA Recombination and Plasma Cell Differentiation. Front Immunol. 2015;6:627.

246. West JA, Viswanathan SR, Yabuuchi A, et al. A role for Lin28 in primordial germ-cell development and germ-cell malignancy. Nature. 2009;460(7257):909-913.

247. Nie K, Zhang T, Allawi $\mathrm{H}$, et al. Epigenetic down-regulation of the tumor suppressor gene PRDM1/Blimp-1 in diffuse large B cell lymphomas: a potential role of the microRNA let-7. Am J Pathol. 2010;177(3):1470-1479.

248. Leucci E, Onnis A, Cocco M, et al. B-cell differentiation in EBV-positive Burkitt lymphoma is impaired at posttranscriptional level by miRNA-altered expression. Int J Cancer. 2010;126(6):1316-1326.

249. O'Carroll D, Mecklenbrauker I, Das PP, et al. A Slicer-independent role for Argonaute 2 in hematopoiesis and the microRNA pathway. Genes Dev. 2007;21(16):1999-2004.

250. Koralov SB, Muljo SA, Galler GR, et al. Dicer ablation affects antibody diversity and cell survival in the B lymphocyte lineage. Cell. 2008;132(5):860-874. 
Aypothesis \& Alims 

The transition from B cells to plasma cells involves dramatic transcriptional modifications that result in the plasma cell phenotype, characterized by long-term survival, and the production and secretion of antibodies. Defects in plasma cell development can seriously impact on human health. Indeed, in several lymphoid neoplasms, the disruption of the B cells differentiation process, such as mutations of certain key B cell maturation factors has been associated with the malignant transformation of B cell.

Although the understanding of the molecular bases that regulate plasma cell formation has progressed in the last years, little is known about the mechanisms involved in the maintenance of long-lived plasma cells, which are the cells that abnormally accumulates in the bone marrow of patients with multiple myeloma. Increasing knowledge of the factors involved in the maturation of plasma cell could help us understand the pathogenesis of multiple myeloma, and thereby provide new perspectives for the development of more effective therapeutic strategies that could block the deleterious activity of plasma cells in multiple myeloma.

Using microarray expression analysis, we had found in a previous study that the mRNA encoding DEPTOR and IRE1 were overexpressed in normal plasma cells and myeloma cells compared with normal B lymphocytes. For this reason, we hypothesized that the variable expression of DEPTOR and IRE1 proteins could contribute to induce different maturation states of myeloma cells. DEPTOR is an mTOR inhibitor that has been found to be overexpressed in a subset of multiple myeloma harboring $C C N D 1 / C C N D 3$ or $M A F / M A F B$ translocations. In these cells, high DEPTOR expression was found necessary to maintain PI3K and AKT activation and the reduction in DEPTOR levels led to apoptosis. However, a putative role of DEPTOR in plasma cell maturation has not previously been reported. The endoplasmic reticulum stress sensor IRE1 has been demonstrated to be required during early and late B cell differentiation. On one side, it is critical at pro-B cell stage for proper VDJ recombination of Ig genes, and on the other side, IRE1 has been shown to be indispensable for terminal differentiation of B cells into plasma cells through the activation of XBP1.

Over the last years, numerous miRNAs have been found to be involved in B cell maturation. This fact prompted us to reason that the expression of both DEPTOR and IRE1 could also be regulated at post-transcriptional level by miRNAs.

In addition to participating in plasma cell maturation, IRE1 has also been shown to target several mRNAs for degradation in an XBP1-independent manner. This mechanism known as regulated IRE1-dependent decay (RIDD) may depend on the nature of the stress stimuli and the tissue context. Several studies have suggested that many RIDD targets are yet to be identified. In myeloma cells, the only RIDD target that has already been identified is BLOC1S1 
mRNA. Our hypothesis was that many other RIDD targets might exist in multiple myeloma and some of them could regulate the survival or proliferation of myeloma cells.

\section{Aims:}

1) To investigate the role of DEPTOR gene in the terminal differentiation of myeloma cells.

- To study the effects of DEPTOR silencing in the maturation state of myeloma cells.

- To identify miRNAs that modulate DEPTOR expression in multiple myeloma and to ascertain their effect on plasma cell maturation.

- To correlate the clinical outcome of multiple myeloma patients with the levels of DEPTOR protein.

2) To uncover the function of IRE1 in the maturation of myeloma cells.

- To analyze the impact of IRE1 knockdown in the maturation state of myeloma cells.

- $\quad$ To explore the epigenetic regulation of IRE1 by microRNAS.

3) To identify regulated IRE1-dependent decay (RIDD) targets in multiple myeloma by RNAsequencing.

- To analyze the presence of IRE1 cleavage site in RIDD targets.

- To investigate whether the identified targets are degraded upon the induction of endoplasmic reticulum stress. 


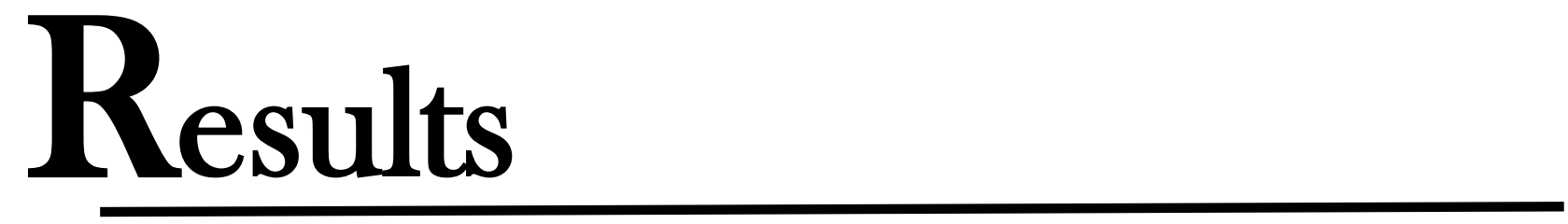

This section includes the experimental work conducted in this thesis, including Material and Methods, Results, Discussion, and Supplementary materials for each chapter.

1. Chapter I: DEPTOR maintains plasma cell differentiation and favorably affects prognosis in multiple myeloma

2. Chapter II: MiR-124 and miR-506 overexpression promote plasma cell dedifferentiation through the regulation of IRE1

3. Chapter III: RNA sequencing identifies novel regulated IRE1-dependent decay targets that affect multiple myeloma survival and proliferation 



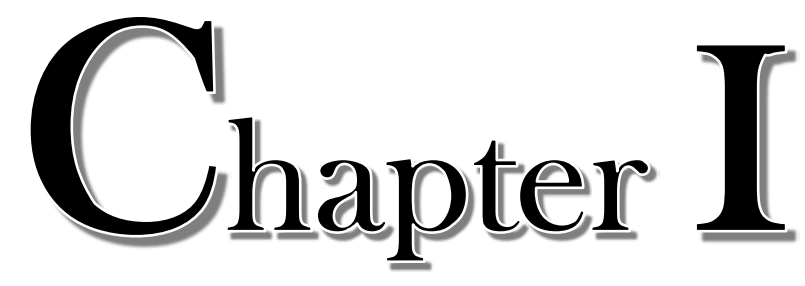

\section{DEPTOR maintains plasma cell differentiation and favorably affects prognosis in multiple myeloma}

Dalia Quwaider $^{1.3}$, Luis A. Corchete ${ }^{1,3}$, Irena Misiewicz-Krzeminska ${ }^{1,3}$, María E. Sarasquete ${ }^{1,3}$, José J.

Pérez, Patryk Krzeminski1,3, Noemí Puig ${ }^{1.2 .3}$, María Victoria Mateos ${ }^{1.2 .3}$, Ramón García-Sanz, Ana B.

Herrero $^{1,3 \dagger}$, and Norma C. Gutiérrez ${ }^{1,2,3 *}$

$\dagger$ Equal contributors

'Cancer Research Center-IBMCC (USAL-CSIC), Salamanca, Spain; 'Hematology Department,

University Hospital of Salamanca, Spain; ${ }^{3}$ Institute of Biomedical Research of Salamanca

(IBSAL), Spain; ${ }^{4}$ National Medicines Institute, Warsaw, Poland.

J Hematol Oncol. 2017 Apr 18;10(1):92. doi: 10.1186/s13045-017-0461-8.

PMID: 28420429 



\section{Abstract}

Background: The B cell maturation process involves multiple steps, which are controlled by relevant pathways and transcription factors. The understanding of the final stages of plasma cell (PC) differentiation could provide new insights for therapeutic strategies in multiple myeloma (MM). Here, we explore the role of DEPTOR, an mTOR inhibitor, in the terminal differentiation of myeloma cells, and its potential impact on patient survival.

Methods: The expression level of DEPTOR in MM cell lines and B cell populations was measured by real-time RT-PCR, and/or Western blot analysis. DEPTOR protein level in MM patients was quantified by capillary electrophoresis immunoassay. RNA interference was used to downregulate DEPTOR in MM cell lines.

Results: DEPTOR knockdown in H929 and MM1S cell lines induced dedifferentiation of myeloma cells, as demonstrated by the upregulation of PAX5 and BCL6, the downregulation of IRF4 and a clear reduction in cell size and endoplasmic reticulum mass. This effect seemed to be independent of mTOR signaling, since mTOR substrates were not affected by DEPTOR knockdown. Additionally, the potential for DEPTOR to be deregulated in MM by particular miRNAs was investigated. The ectopic expression of miR-135b and miR-642a in myeloma cell lines substantially diminished DEPTOR protein levels and caused dedifferentiation of myeloma cells. Interestingly, the level of expression of DEPTOR protein in myeloma patients was highly variable, the highest levels being associated with longer progression-free survival.

Conclusion: Our results demonstrate for the first time that DEPTOR expression is required to maintain myeloma cell differentiation and that high level of its expression are associated with better outcome.

Primary samples used in this study correspond to patients entered into GEM2010 trial (registered at www.clinicaltrials.gov as \#NCT01237249, 4 November 2010). 



\section{Introduction}

Multiple myeloma (MM) is a clonal disorder of B-cells (BCs) in the final stage of differentiation that accounts for approximately $10 \%$ of all hematological cancers ${ }^{1}$. MM is characterized by clonal accumulation of malignant plasma cells (PCs) in the bone marrow, which secrete a monoclonal immunoglobulin. Although several therapeutic agents are available, MM remains incurable. Knowledge of all the factors involved in PC differentiation could provide new insights of relevance to therapeutic strategies for MM. In fact, in some hematological neoplasms, the malignant transformation of $\mathrm{BC}$ has been associated with the disruption of the B cell differentiation process, such as mutations of certain key BC maturation factors ${ }^{2-4}$. The transition from $\mathrm{B}$ lymphoid precursors to antibody-secreting PCs involves several molecular and cellular modifications including transcriptional changes, expansion of the cytoplasm and the secretory organelles to accommodate high-rate synthesis of immunoglobulins, unfolded protein response (UPR) activation and changes in cell surface antigen expression ${ }^{5-8}$. It has been demonstrated that the transcriptomes of $\mathrm{BC}$ and PC are maintained by two groups of transcriptional factors: those that promote the $\mathrm{B}$ cell program, such as PAX5, BCL6 and BACH2, and those that favor and facilitate PC differentiation, notably IRF4, BLIMP1 and XBP1 9 . Interestingly, many of these transcription factors repress others required for the alternative developmental state, thereby establishing mutually exclusive gene-expression programmes ${ }^{9-12}$. Besides transcriptional factors, other types of proteins and biochemical pathways could be involved in the transformation of $\mathrm{BC}$ into mature PC.

Using microarray expression data, we found that the mRNA encoding DEPTOR, an inhibitor of mTORC1 and mTORC2 kinases activities ${ }^{13}$ was overexpressed in normal PCs (NPCs) and myeloma cells compared with normal B lymphocytes (NBLs) ${ }^{14}$, which raised the possibility that this protein contributes to PC differentiation. The complete role of DEPTOR within the cells has not yet been fully elucidated, although the involvement of DEPTOR in several biological processes, such as cell growth, apoptosis, autophagy has been reported ${ }^{15}$. A potential role of DEPTOR as a tumor suppressor or as an oncogene, depending on cell context, has been reported. It is considered a tumor suppressor, functioning by the inhibition of mTOR, whose activity is frequently hyperactivated in many human tumors. Indeed, DEPTOR has been found to be downregulated in many types of human cancers. However, it is also overexpressed in many other tumor types, including chronic myeloid leukemia, and MM 13,16. The overexpression of DEPTOR in MM has been associated with translocations involving MAF transcription factors and CCND1 and CCND3 genes ${ }^{13}$. 
Furthermore, DEPTOR seems to be overexpressed in MM with copy number gains of 8q24 where DEPTOR is located ${ }^{17}$.

Here, we report for the first time that DEPTOR maintains the terminal differentiation of MM cells. Knockdown of DEPTOR reverts the transcriptional program of the PC to that characteristic of a BC. In addition, we found that microRNA deregulation in $\mathrm{MM}$, specifically miR-642a and miR-135b downregulation may also underpin the overexpression of DEPTOR. 


\section{Materials and methods}

\section{Cell lines and primary samples}

The human multiple myeloma cell lines (MMCL), NCI-H929, MM1S and U266 were acquired from the ATCC (American Type Culture Collection), and the JJN3, RPMI-8226, OPM-2, KMS12BM, KMS12PE and HEK923 lines were obtained from the Deutsche Sammlung von Mikroorganismen and Zellkulturen (DSMZ). Cell line identity was confirmed periodically by STR analysis with the PowerPlex 16 HS system kit (www.promega.com) and online STR matching analysis (www.dsmz.de/fp/cgi-bin/str.html). Cell lines were cultured in RPMI 1640 medium supplemented with 10\% fetal bovine serum and antibiotics (Gibco Life Technologies, Grand Island, NY, USA). Bone marrow (BM) samples from ten healthy donors were sorted by a FACSAria equipment into four BC populations: immature B cells (CD34-, CD19+, CD10+, CD38++), naive B cells (CD19+, CD27-, CD10-), memory B cells (CD19+, CD138-, CD27+, CD38+) and plasma cells (CD38+++, CD138+, CD45low). Monoclonal antibodies were purchased as follows: anti-CD45-FITC (clone D3/9), and anti-CD19-PECy7 (clone A3-B1) from Immunostep (Salamanca, Spain); anti-CD38-PerCP-Cy ${ }^{\mathrm{TM}} 5.5$ (clone HIT2), anti-CD34-APC (clone 8G12), and anti-CD27-BV421 (clone M-T271) from BD Biosciences (San Jose, CA, USA); anti- CD138-Pacific Orange ${ }^{\mathrm{TM}}$ (clone B-A38) from Exbio Praha (Vestec, Czech Republic); and anti-CD10-PE (clone ALB1) from Beckman Coulter (Pasadena, CA, USA). CD138+ plasma cells were isolated from BM samples of 24 patients with newly diagnosed MM included in the GEM2010 Spanish trial (bortezomib, melphalan and prednisone plus lenalidomide and dexamethasone), using an autoMACS separation system (Miltenyi-Biotec, Auburn, CA, USA).

\section{RNA extraction and quantitative real-time PCR analysis}

RNA was extracted from the cell lines using an RNeasy mini kit (Qiagen, Valencia, USA) according to the standard protocol. RNA integrity was assessed using an Agilent 2100 Bioanalyzer (Agilent Technologies, Santa Clara, CA, USA). Total RNA (1 $\mu \mathrm{g})$ was reversetranscribed to cDNA using High-Capacity cDNA Reverse Transcription Kits (Applied Biosystems, Foster City, CA, USA). Expression of target genes was assessed using TaqMan qRT-PCR assays (Applied Biosystems). Relative gene expression was calculated by the 2-ACt method using GAPDH as the endogenous control for normalization.

To detect mature miR-135b and miR-642a expression levels, TaqMan quantitative real-time polymerase chain reaction (qRT-PCR) miRNA assay (Applied Biosystems) was performed. The relative levels of expression of mature miR-135b and miR-642a normalized with respect to the RNU43 endogenous control were determined by the $2^{-\Delta C t}$ method. Each measurement was performed in triplicate. 


\section{Transfections}

Cell lines were transfected using the nucleofector II system (Lonza, Allendale, NJ, USA) with the following programs: C-16 for H929 and JJN3, G-16 for MM1S, and X-005 for U266. Cells were transfected with on-TARGET plus ${ }^{\text {TM }}$ control pool or on-TARGET plus SMART pool Human DEPTOR (Dharmacon, Lafayette, CO, USA); pre-miR ${ }^{\mathrm{TM}}$ miRNA precursors pre-miR135b, pre-miR-642a, and pre-miR ${ }^{\mathrm{TM}}$ miRNA negative non-targeting control\#1 (Ambion, Austin, TX, USA); and microRNA inhibitors, hsa-miR-135b-5p miRCURY LNA ${ }^{\mathrm{TM}}$ microRNA inhibitor, hsa-miR-642a-5p miRCURY LNA ${ }^{\mathrm{TM}}$ microRNA inhibitor and miRCURY LNA ${ }^{\mathrm{TM}}$ microRNA inhibitor negative control A (Exiqon, Woburn, MA, USA). siRNA and miRNA concentration of $25 \mathrm{nM}$ was used in all the experiments.

\section{Cell cycle analysis}

Cells were washed in PBS and fixed in 70\% ethanol for later use. Cells were rehydrated with PBS, resuspended in $500 \mu \mathrm{l}$ of PI/RNase staining solution (Immunostep), and incubated for 20 minutes at RT in the dark. Samples were analyzed using a FACSCalibur flow cytometer.

\section{Apoptosis and cell proliferation assays}

Apoptosis was measured using an annexin V-fluorescein isothiocyanate/propidium iodide (PI) double staining (Immunostep) according to the manufacturer's procedure. Cell viability was evaluated with the CellTiter-Glo® luminescent cell viability assay based on the amount of ATP present (Promega), in accordance with the manufacturer's protocol.

\section{Immunophenotyping}

MM cell lines were immunophenotyped on a FACSCanto II cytometer (Beckton Dickinson Biosciences) using the following monoclonal antibodies: CD138-OC515 (Cytognos S.L., Salamanca, Spain), CD38-APC-H7 (BD Biosciences) and sIgk-PB (Vestec, Czech Republic). Data analysis was performed using the Infinicyt software (Cytognos S.L.). A minimum of $10^{5}$ events were stored. Median fluorescence intensity of each marker was analyzed.

\section{Luciferase reporter assay}

The double-stranded oligonucleotides corresponding to the wild-type or mutant miR-135b and miR-642a binding sites in the $3^{\prime}$-untranslated region (3'UTR) of DEPTOR were synthesized (Supplementary materials: Table S1) (Sigma-Aldrich, St Louis, MO, USA) and ligated between the PmeI and Xbal restriction sites of the pmirGLO vector (Promega, Madison, WI, USA). Oligonucleotide sequences are detailed in (Supplementary materials: Table S1). Luciferase assays in HEK293 cells were performed as previously described 18 . 


\section{Western blot}

Protein extraction and Western blot analysis were carried out as previously detailed ${ }^{18}$. The primary antibodies used for immunoblotting were anti-DEPTOR, anti-AKT, phospho-AKT (ser473), anti-p70 S6 kinase, anti-phospho-p70 S6 (Thr389), anti-4E-BP1, anti-phospho4E-BP1 (Thr37/46), anti-phosphor-S6 ribosomal protein (Ser235/236) (Cell Signaling Technology, Beverly, MA, USA), anti-IRF4, anti-Ig kappa light chain and anti-Ig lambda light chain (Santa Cruz Biotech, Delaware, CA, USA). Anti- $\beta$-actin (Sigma-Aldrich) was used as an internal control for protein loading. The membranes were then washed and incubated with the secondary horseradish per-oxidase-linked anti-mouse IgG or anti-rabbit IgG antibodies (PierceNet) (1:10000), anti-goat IgG (Santa Cruz Biotech) (1:10000). Chemiluminescence was detected using the Amersham ECL Plus ${ }^{\text {m }}$ Western Blotting Detection Reagent (GE Healthcare).

\section{Capillary electrophoresis immunoassay}

Capillary electrophoresis immunoassay was performed using the WES $^{\mathrm{TM}}$ machine (ProteinSimple Santa Clara, CA, USA) according to the manufacturer's protocol. In brief, $4 \mu \mathrm{l}$ of samples at a concentration of $0.1 \mathrm{mg} / \mathrm{ml}$ (or lower when it was not possible to achieve $0.1 \mathrm{mg} / \mathrm{ml}$ ) were combined with a master mix (ProteinSimple) to a final concentration of $1 \mathrm{x}$ sample buffer, $1 \mathrm{x}$ fluorescent molecular weight markers, and $40 \mathrm{mM}$ dithiothreitol (DTT), and then heated at $95^{\circ} \mathrm{C}$ for $5 \mathrm{~min}$. The samples, blocking reagent, wash buffer, primary antibodies (anti-DEPTOR and anti-GAPDH at 1:100 concentration), secondary antibodies, and chemiluminescent substrate were dispensed into designated wells in the microplate provided by the manufacturer. After plate loading, the fully automated separation electrophoresis and immunodetection steps were carried out in the capillary system. Data were analyzed with the inbuilt Compass software (ProteinSimple). The signal from DEPTOR was normalized with respect to the signal from GAPDH, making sure that the signals of both proteins were within the linearity range.

\section{Immunofluorescence staining}

Cells were collected 48 or 72 h post-transfection, washed with PBS, and stained for $30 \mathrm{~min}$ with $1 \mu \mathrm{M}$ ER-Tracker ${ }^{\mathrm{TM}}$ Red (Invitrogen). Cells were washed again with PBS, fixed with 4\% formaldehyde for $5 \mathrm{~min}$ at room temperature and placed on glass slides coated with polyL-lysine and stained for $1 \mathrm{~min}$ with DAPI II. The slides were then mounted using VECTASHIELD Mounting Medium (Vector Laboratories, Burlingame, CA, USA). Images were collected under a Zeiss confocal microscope equipped with 636/1.4 Oil Plan-APOCRHOMAT DIC. 


\section{Statistical analysis}

The two-tailed Student's t-test or the two-tailed Welch's t-test was used to analyze group differences in experiments when data showed equal or unequal variances, respectively. Data are reported as mean values \pm standard deviation (SD) of at least three determinations. Progression-free survival (PFS) distribution curves were plotted using the Kaplan-Meier method; the log-rank test was used to estimate the statistical significance of differences between the curves. The Cutoff Finder web application (http://molpath.charite.de/cutoff) was used to determine the optimal cutoff, defined as that yielding the most significant split discriminating shorter and longer survival, and identified by testing all possible cutoffs using the log-rank test ${ }^{19}$. Values of $p<0.05$ were considered statistically significant. All statistical analyses were conducted using the SPSS 21.0 program (IBM Corp. Released 2012. IBM SPSS Statistics for Windows, Version 21.0. Armonk, NY: IBM Corp). 


\section{Results}

\section{DEPTOR is overexpressed in plasma cells compared with B lymphocytes.}

In order to confirm the previously observed overexpression of DEPTOR by microarray analysis in NPC and myeloma cells relative to NBL (GSE6691 at GEO repository) ${ }^{14}$ (Figure 1A), we quantified DEPTOR mRNA levels by qRT-PCR in four BC populations. To this end, immature, naïve, memory B cells and PCs were sorted from BM samples obtained from healthy donors. DEPTOR expression was found to be significantly higher in PCs than in all previous stages of differentiation (Figure 1B), which suggested that this protein could be involved in PC maturation.

A

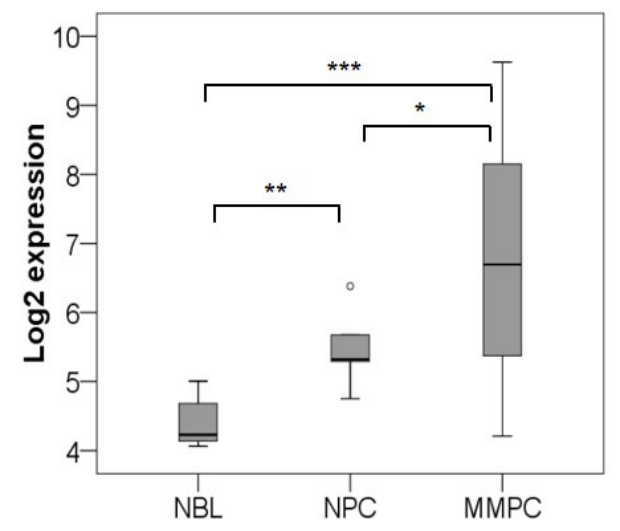

B

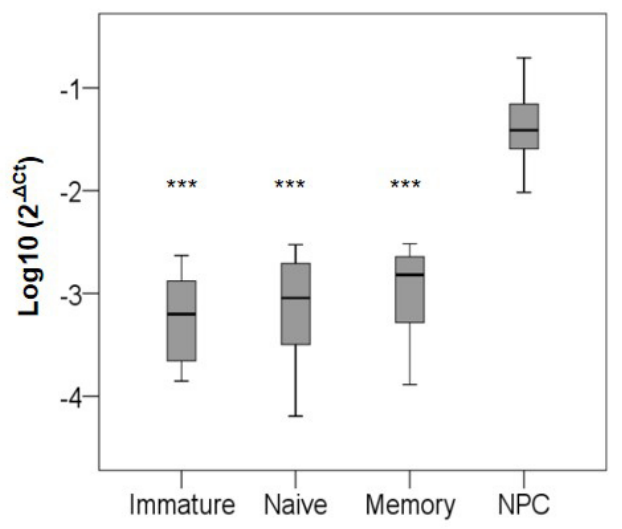

Figure 1. DEPTOR expression. A DEPTOR expression in normal B lymphocytes (NBL), normal PC (NPC), and myeloma cells obtained from gene expression arrays (GSE6691 at GEO repository). B DEPTOR expression detected by qRT-PCR in immature B cells, naive B cells, memory B cells and NPC. $\left({ }^{*} p<0.05,{ }^{* *} p<0.01,{ }^{* * *} p<0.001\right)$. 


\section{DEPTOR knockdown induces dedifferentiation of myeloma cells}

To gain further insight into the potential role of DEPTOR in PC differentiation we knocked down its expression in MMCLs by siRNA for $48 \mathrm{~h}$ and then assessed the expression of key genes involved in B cell maturation by qRT-PCR. Cell growth and apoptosis experiments revealed that DEPTOR knockdown did not alter cell viability (Supplementary materials: Figure S $1 \mathrm{~A}$ and 1B). However, we found that PAX5 and BCL6, which both encode B-cell lineage-specific activator proteins and which are present only at early stages of B-cell differentiation ${ }^{9}$, were increased in DEPTOR-silenced cells (Figure 2A). Conversely, IRF4, an essential transcription factor for PC differentiation ${ }^{20}$, was downregulated after DEPTOR knockdown. Reduced IRF4 levels were also confirmed by Western blot, mainly in H929 cells (Figure 2B). Additionally, immunophenotypic markers related to B-cell differentiation, such as CD19, CD38, CD138, and k light chain were also assessed. Thus, expression levels of CD38, CD138, and $\mathrm{k}$ light chain were found to be lower, while CD19 expression was higher after DEPTOR silencing (Figure 2A, 2B, and 2C). Next, we analyzed the effect of DEPTOR downregulation on myeloma cell morphology. Clear reductions in cell size and endoplasmic reticulum mass were found in both H929 and MM1S as a consequence of DEPTOR downregulation (Figure 3A, 3B, 3C). To exclude the possibility that cell cycle profiles were responsible for the differences in size of myeloma cells, we determined the percentage of cells in G1, S and G2/M by flow cytometry. No differences in cell cycle profiles were observed between control and DEPTOR-silenced cells (Figure. 3D). Next, we also determined whether the observed reduction in cell size in DEPTOR-silenced cells could also be detected by flow cytometry. A clear reduction in mean FSC value was observed in DEPTOR-silenced cells compared with control cells (Figure 3E). Taken together, these results indicate that DEPTOR downregulation in MM cells induces a reversal of PCs to previous stages of PC differentiation. 
A
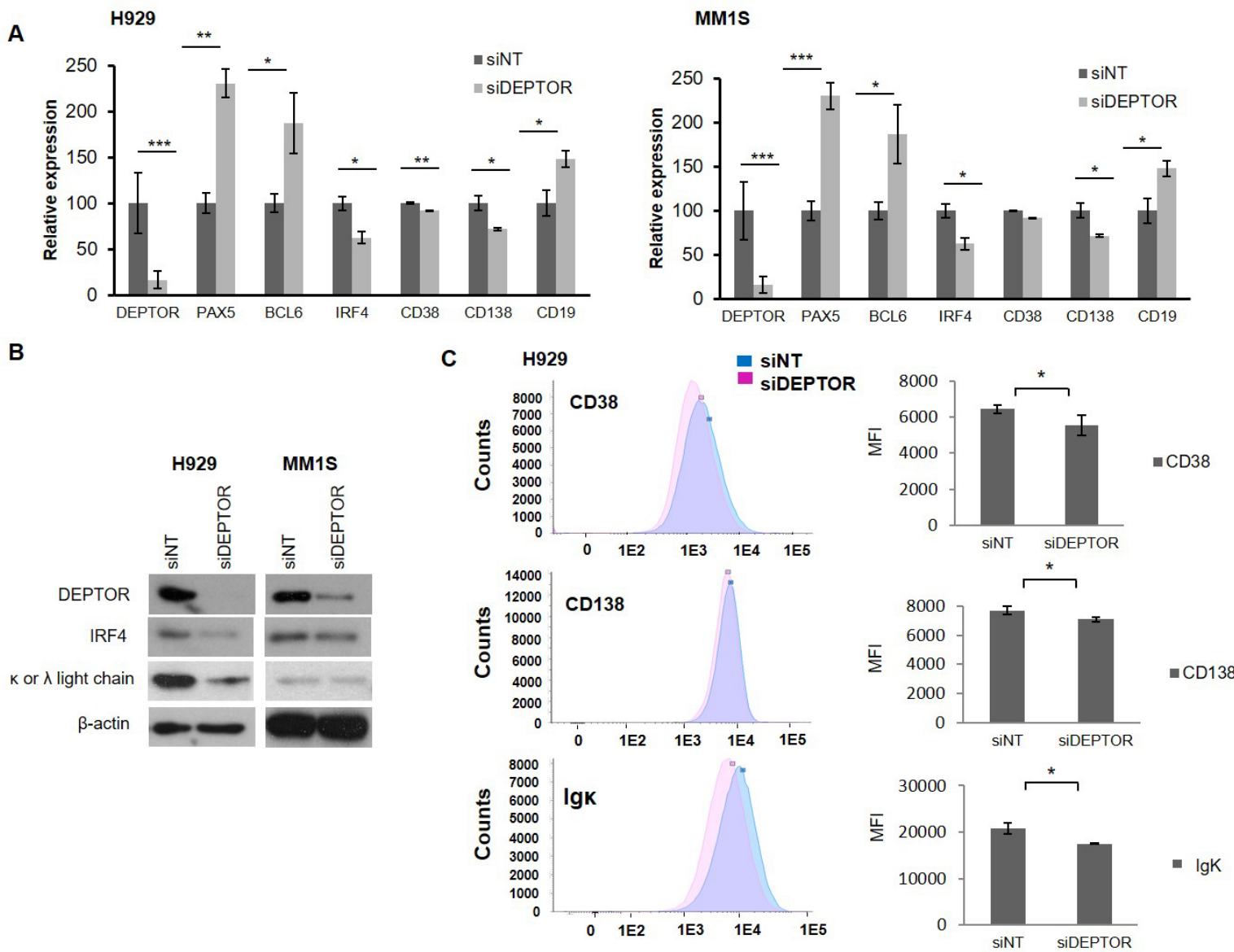

Figure 2. DEPTOR knockdown modulates transcription factors and markers related to B cell maturation. A mRNA levels of the indicated genes in H929 and MM1S determined by qRT-PCR $48 \mathrm{~h}$ after DEPTOR knockdown. B Western blot of DEPTOR, IRF4, kappa and lambda light chain in H929 and MM1S. C Flow cytometry analysis of CD38, CD138, and cytoplasmic kappa light chain in H929 after DEPTOR knockdown (siDEPTOR) compared with non-targeting control (siNT); left panel: representative histograms; right panel: mean fluorescence intensity values (MFI). All results are presented as the means \pm SD of three different experiments. $\left({ }^{*} p<0.05,{ }^{* *} p<0.01,{ }^{* * *} p<0.001\right)$. 
A H929

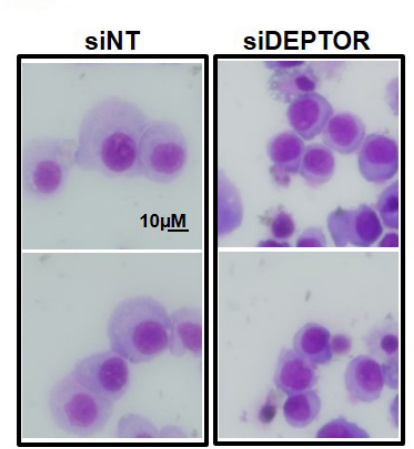

B

H929

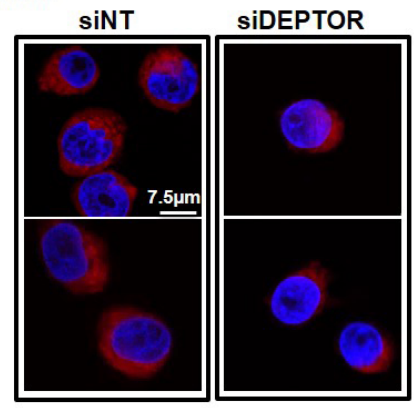

MM1S

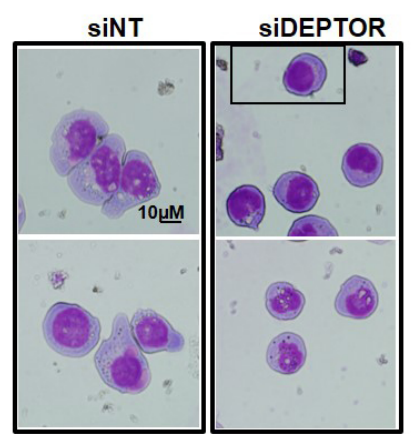

MM1S

siNT

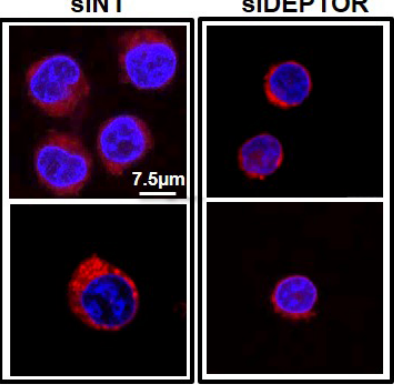

C
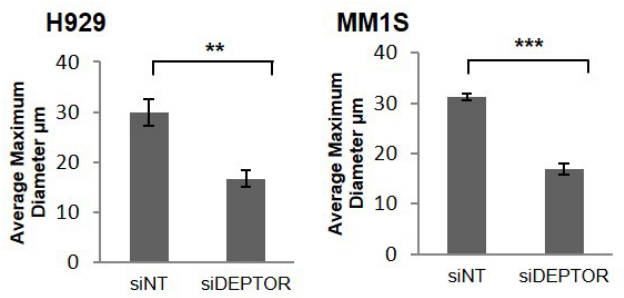

D

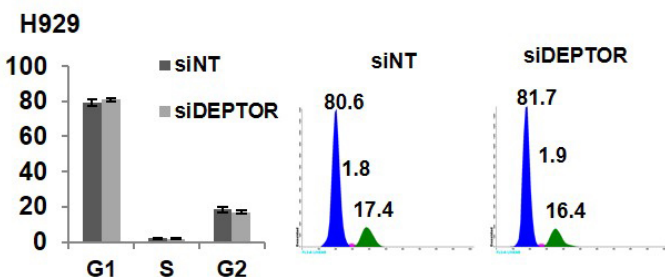

E

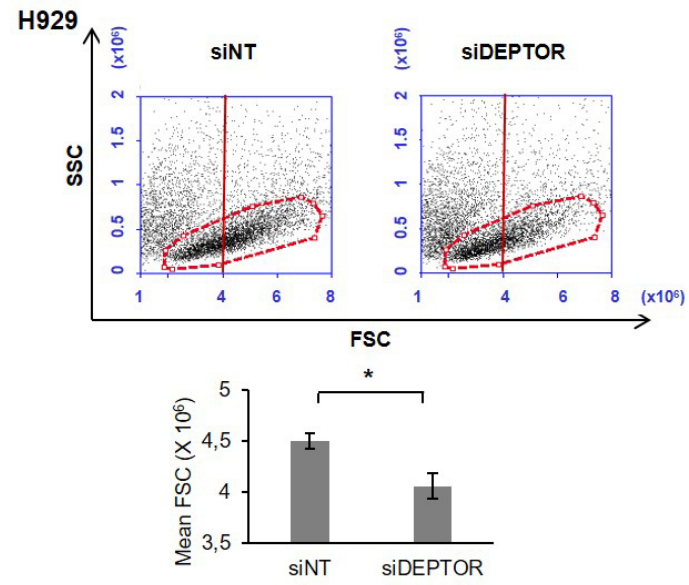

Figure 3. DEPTOR knockdown induces dedifferentiation of myeloma cells. A Giemsa stain of H929 and MM1S after DEPTOR knockdown. B Immunofluorescence of H929 and MM1S cells stained with ER-Tracker ${ }^{\mathrm{TM}}$ Red dye. C Average maximum diameter of H929 and MM1S transfected with nontargeting (siNT) and DEPTOR siRNA (siDEPTOR) measured from two independent experiments. At least 100 cells per experiment were counted. D Cell cycle analysis $48 \mathrm{~h}$ after DEPTOR knockdown. The percentages of cells in different phases of the cell cycle are indicated. E Cell size of H929 48 h after DEPTOR knockdown. Representative dot plots showing forward-scatter (FSC) versus sidescatter (SSC). All results are presented as the means \pm SD of three different experiments. $(* p<0.05$, ** $\mathrm{p}<0.01, * * * \mathrm{p}<0.001)$. 


\section{Dedifferentiation of myeloma cells induced by DEPTOR silencing is independent of mTOR signaling}

As DEPTOR has been described previously as an mTORC1/mTORC2 inhibitor ${ }^{13}$, we were interested to determine whether PC dedifferentiation observed after DEPTOR silencing was induced through the mTOR signaling pathway. For this purpose, mTORC1 and mTORC2 activity was monitored through the phosphorylation state of their substrates using the same conditions of DEPTOR knockdown that induced PC de-differentiation. We found that the levels of mTOR substrates were not changed by DEPTOR knockdown under the conditions assayed (Figure 4A). These results implied that all the molecular and morphological changes obtained after DEPTOR knockdown were independent of the mTOR pathway. To confirm these findings, we added rapamycin, a well-known mTORC1 inhibitor, in the DEPTOR knockdown experiments. As shown in (Figure 4B), levels of p-S6 were again found to be similar in control and in DEPTOR knockdown cells, but lower in cells exposed to rapamycin, showing that the downregulation of IRF4 triggered by DEPTOR silencing was not reverted by the addition of rapamycin. Therefore, the PC state is maintained by DEPTOR independent of its role as an mTORC inhibitor.

A

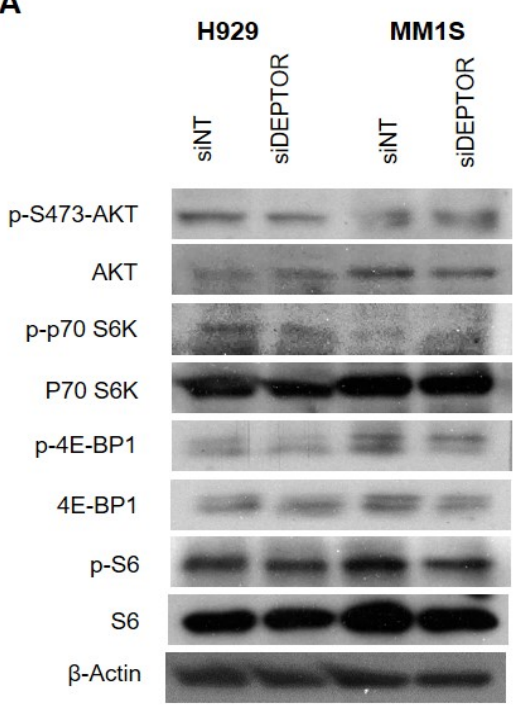

B

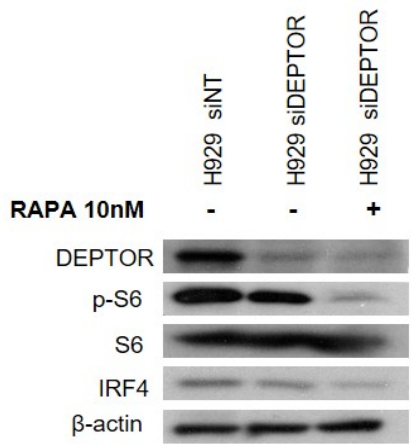

Figure 4. Dedifferentiation of myeloma cells after DEPTOR silencing is independent of mTOR signaling. A Levels of mTORC1 and mTORC2 substrates after DEPTOR knockdown in H929 and MM1S. All experiments were carried out $48 \mathrm{~h}$ post-transfection. B Western blot of $\mathrm{H} 929$ transfected with siNT or siDEPTOR and cultured with control medium, and rapamycin at $10 \mathrm{nM}$. 


\section{DEPTOR is a direct target of miR-135b and miR-642a}

It has previously been shown that DEPTOR is overexpressed in the subset of MM carrying $M A F / M A F B$ and $C C N D 1 / C C N D 3$ translocations. The influence of MAF/MAFB on DEPTOR levels was confirmed by both ectopic expression of MAFB and MAF silencing, which induced DEPTOR upregulation and downregulation, respectively ${ }^{13}$. We evaluated DEPTOR expression in a panel of MM cell lines with different chromosomal translocations (Figure 5A). DEPTOR mRNA and protein levels were detected in almost all cell lines analyzed, especially in RPMI-8226 and MM1S, which overexpress MAF ${ }^{21}$, but also in H929 and OPM2 carrying the $(4 ; 14)$ translocation. Surprisingly, some MM cell lines exhibited low levels of DEPTOR, like JJN3, even though it carries a MAF translocation, and U266, which overexpress cyclin D1 22-26. These results suggest that, although DEPTOR levels may be influenced by MAF/MAFB or cyclin D1/3 expression, additional mechanisms may affect its expression. To address the possibility that post-transcriptional regulation by microRNAs is involved in DEPTOR expression, we looked for miRNA-DEPTOR interactions in five databases (miRMap, PITA, RNA22, RNAhybrid and Targets scan), using a value of $p=0.05$ for miRNAs whose predicted binding site is the 3'UTR of DEPTOR (Supplementary materials: Figure S2). A total of 47 common miRNAs were predicted to target DEPTOR from the five combined datasets. Of these, miR-135b and miR-642a had been previously reported to be downregulated in MM with different cytogenetic abnormalities ${ }^{27}$. Underexpression of both miRNAs in different MM patients compared with NPCs was confirmed by qRT-PCR (Figure 5B). According to the prediction algorithms, miR-642a and miR-135b have one putative site in the DEPTOR $3^{\prime} \mathrm{UTR}$ (Figure 5C). To determine whether DEPTOR was a direct target of those miRNAs, we carried out luciferase reporter assays. The 3'UTR of DEPTOR harboring the sequence complementary to the miR-642a or miR-135b seed sequence was cloned in a reporter plasmid vector referred to as wild-type (WT). In parallel, a 3'UTR DEPTOR fragment containing mutant sequences (MUT) of the seed site of the two miRNAs was cloned in the same reporter plasmid. DEPTOR 3'UTR WT and MUT luciferase constructs were then transfected into HEK293 cells along with miR-135b/miR-642a or negative control (NC) miRNA, and luciferase activity was determined. We found that luciferase activity of cells cotransfected with WT DEPTOR 3'UTR and miR-135b or miR-642a was significantly lower $(p<0.01)$ than that exhibited by cells transfected with NC control miRNA. Luciferase activity of MUT constructs was not affected by miR-135b or miR-642a overexpression (Figure 5D). 


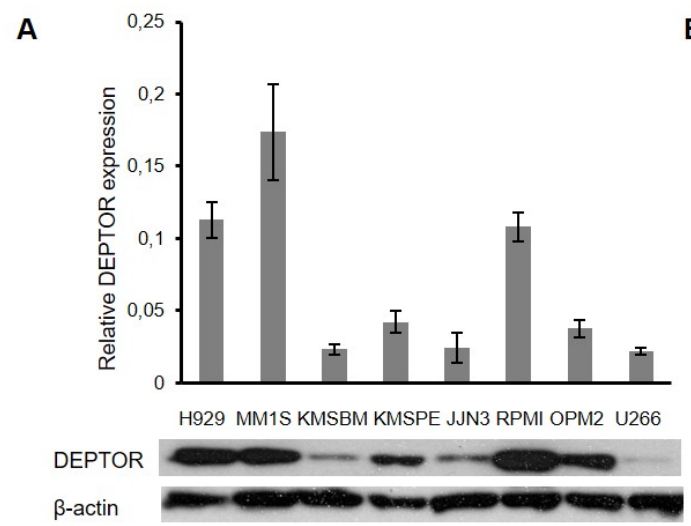

B
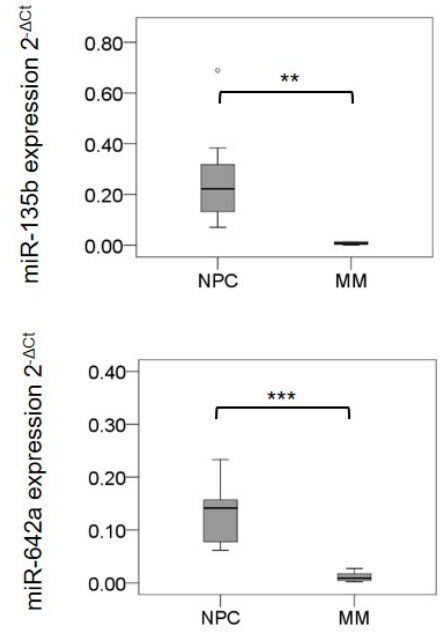

C

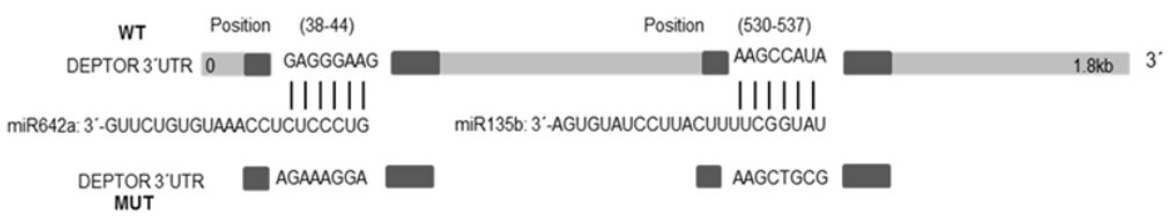

D
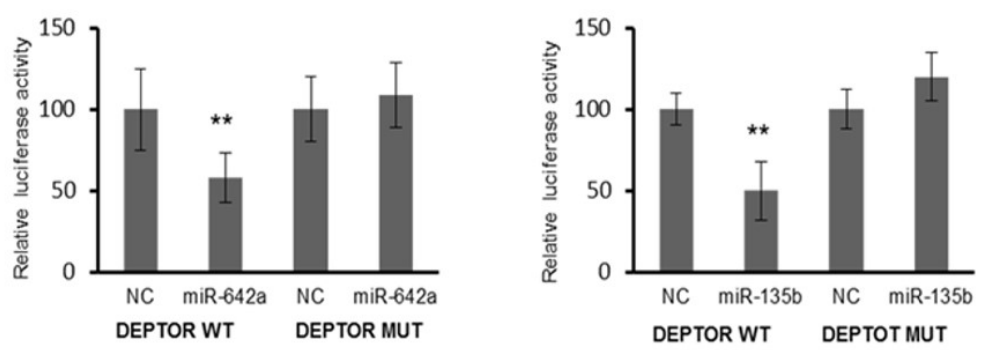

Figure 5. DEPTOR expression is controlled by miR-135b and miR-642a in MM. A DEPTOR mRNA levels measured by qRT-PCR (upper panel). Levels of DEPTOR in MM cell lines detected by Western blot (lower panel). B miR-135b and miR-642a expression in NPC and MM patients measured by qRTPCR. microRNAs expression was normalized to RNU43 $\left(-2^{\Delta c t}\right)$. Two-tailed Welch's $t$-test $p$-values for miR-135b and miR-642a were calculated ( 0.0081 and 0.0001 respectively). C Schematic diagram of the miR-642a and miR-135b predicted site on the DEPTOR 3'UTR, dark squares on DEPTOR 3'UTR WT and DEPTOR 3'UTR MUT represents fragments cloned in pmiRGlo plasmid. D Luciferase activity in HEK293 cells co-transfected with pre-miR-NC or pre-miR-135b/ miR-642a and plasmid pmiR-Glo with the putative miR-135b/miR-642a binding site of DEPTOR cloned downstream of the luciferase reporter gene. Luciferase activity was normalized using Renilla. All results are presented as the means \pm SD of three different experiments. $\left({ }^{*} p<0.05,{ }^{* *} p<0.01,{ }^{* * *} p<0.001\right)$. 


\section{MiR-135b and miR-642a regulate DEPTOR expression in MM}

Once DEPTOR had been validated as being a direct target of miR-642a and miR-135b, we speculated that differences in DEPTOR levels among MM cell lines could be partly related with the endogenous levels of these miRNAs. In fact, using qRT-PCR we found that JJN3 and U266, which exhibited low levels of DEPTOR despite MAF or cyclin D1 overexpression (Figure 6A), expressed higher amounts of miR-642a and miR-135b than H929, which displayed high levels of DEPTOR and carried $t(4 ; 14)$ (Figure 6A). To test this hypothesis, H929 was transfected with miR-135b or miR-642a and NC miRNA precursors. Clearly lower levels of DEPTOR protein expression were found in miR-135b and miR-642a transfected cells compared with control cells $72 \mathrm{~h}$ post-transfection (Figure 6B). To gain further evidence of the relationship between miR-642a/miR-135b expression and DEPTOR levels, miR-642a and miR-135b were specifically knocked down in U266 and JJN3 cell lines. DEPTOR protein expression was significantly higher in both cell lines after transfection with miR-642a/miR-135b inhibitors (Figure 6C). Taken together, these results demonstrate that miR-135b and miR-642a modulate DEPTOR expression through the consensus miR-135b or miR-642a binding sites in DEPTOR 3'UTR. These miRNAs can subsequently participate in the regulation of DEPTOR expression in MM.

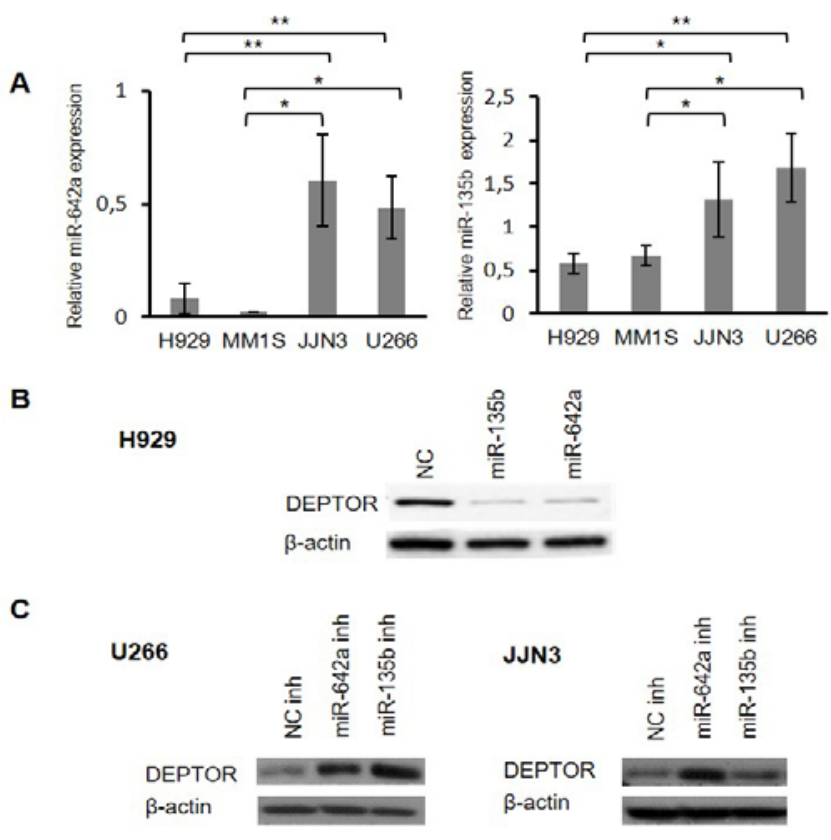

Figure 6. MiR-642a and miR-135b regulate DEPTOR expression in MM. A miR-135b and miR642a expression in MM cell lines measured by qRT-PCR. MicroRNA expression was normalized with respect to RNU43 $\left(-2^{\Delta c t}\right)$. B Western blot of DEPTOR in H929 cells $72 \mathrm{~h}$ post-transfection with NC or miR-135b/miR-642a. C Western blot of DEPTOR in U266 and JJN3 transfected with NC or miR135b/miR-642a inhibitors. $\left({ }^{*} p<0.05,{ }^{* *} p<0.01,{ }^{* * *} p<0.001\right)$. 
Upregulation of miR-135b and miR-642a results in myeloma cell dedifferentiation through the negative regulation of DEPTOR

Next, we were interested to determine whether downregulation of DEPTOR induced by the overexpression of miR-135b or miR-642a also led to PC dedifferentiation. Consistent with the finding after DEPTOR silencing by siRNAs, the downregulation of DEPTOR by transfection of H929 cells with miR-135b or miR-642a resulted in downregulation of IRF4 and $\mathrm{k}$ light chain proteins (Figure 7A). In addition, DEPTOR knockdown by miR-135b and miR-642a overexpression led to the appearance of smaller, rounder cells with a lower cytoplasm and ER content than control cells (Figure 7B and 7C). These results and those obtained from siRNA experiments clearly indicated that the presence of DEPTOR is required to maintain myeloma cells at the terminal stage of differentiation.

A

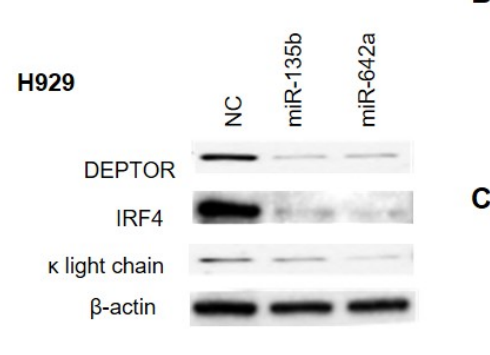

B

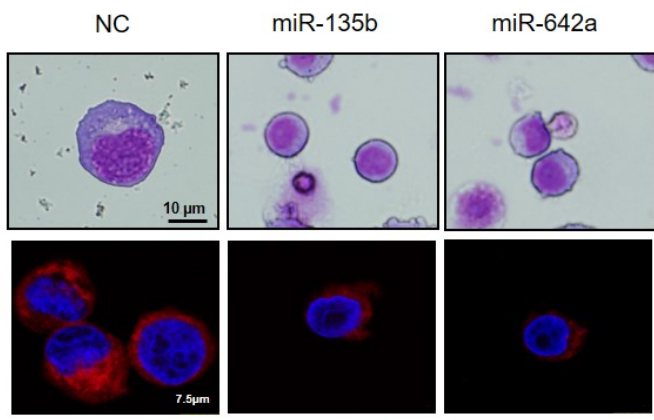

Figure 7. Upregulation of $\mathrm{miR-135b}$ and miR-642a results in myeloma cell dedifferentiation through negative regulation of DEPTOR. A Western blot of DEPTOR, IRF4, kappa light chain in H929 cells 72 h post-transfection with NC or miR-135b/miR-642a. B Giemsa stain of H929 cells transfected with NC or miR-135b/miR-642a. C Immunofluorescence of H929 cells stained with ER tracker. 
DEPTOR is differentially expressed in MM and its upregulation is associated with longer survival and the stage of PC maturity

DEPTOR mRNA is known to be differentially expressed in MM patients 13,28 . We confirmed these results using two different microarray studies (GSE16558, and GSE39925 at GEO repository) 27,29 . Next, we decided to quantify DEPTOR protein levels by capillary electrophoresis immunoassay in myeloma cells from $24 \mathrm{MM}$ patients treated according to the Spanish GEM2010 trial. Consistent with the mRNA expression data, we observed that levels of DEPTOR protein also differed among the myeloma samples (Figure 8A). Interestingly, we found that the PFS was significantly longer in MM patients with high expression levels of DEPTOR than in those with low DEPTOR expression levels $(p=0.038)$ (Figure 8B).

The differences in DEPTOR protein expression among MM patients led us to hypothesize that DEPTOR levels might be associated with the maturation state of myeloma cells of each patient, in line with our results from MMCLs. As morphological characteristics of myeloma cells are correlated with maturation stage ${ }^{30}$, we examined the morphology of myeloma cells isolated from three patients with high DEPTOR protein levels and three with low protein levels. We observed that those cells harboring high levels of DEPTOR had an eccentric nucleus, large and extended cytoplasm, and were bigger than those exhibiting low levels of the protein (Supplementary materials: Figure S3). These results support those obtained from MMCLs and indicate that a suitable level of DEPTOR is necessary for PC maturation. 
A

2,50
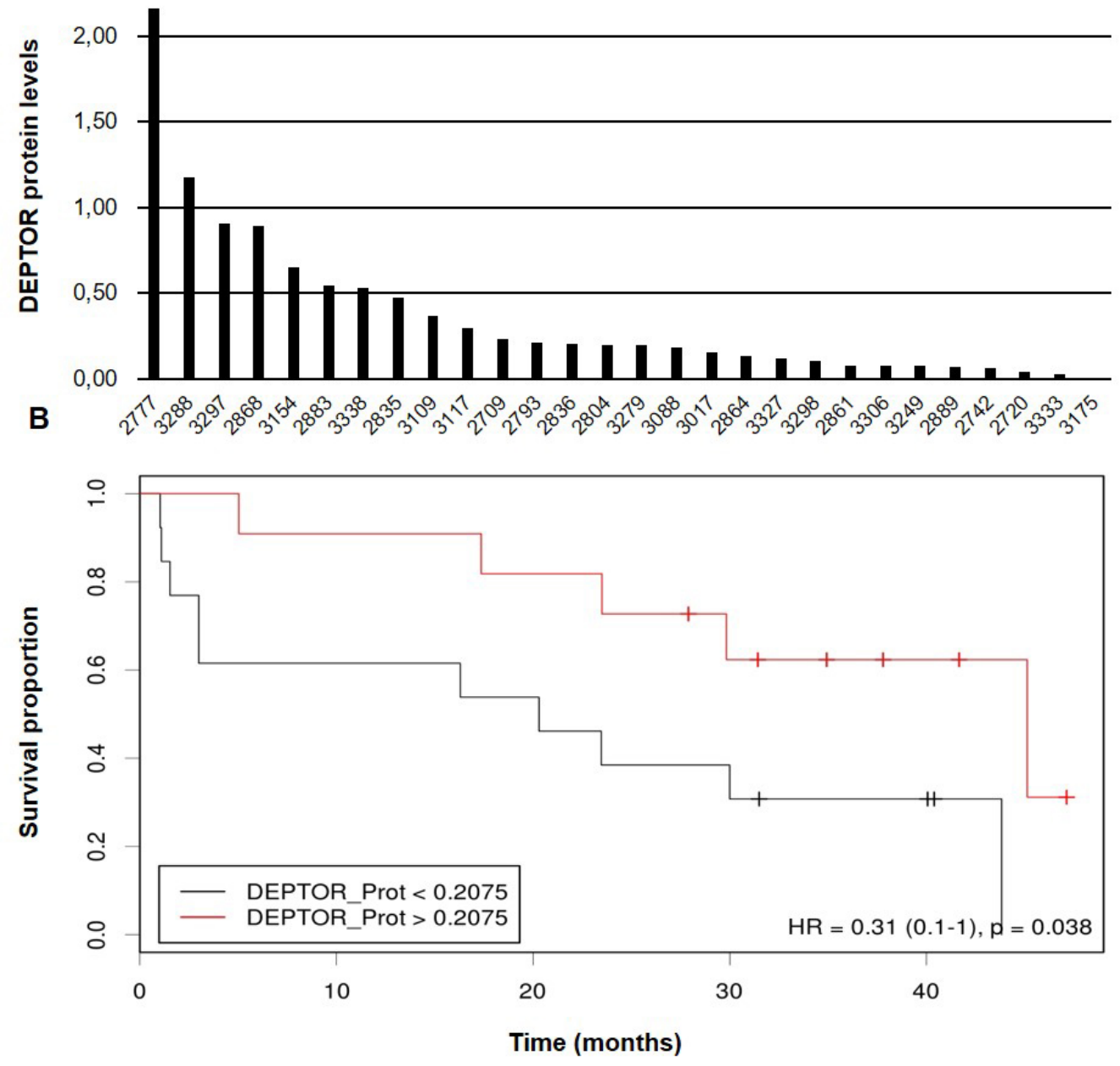

Figure 8. DEPTOR is differentially expressed in MM and its overexpression is associated with longer survival. A Levels of DEPTOR in MM patients assessed using capillary electrophoresis immunoassay. B Kaplan-Meier curves for progression-free survival (PFS) in 24 MM patients treated according to GEM2010 trial. 


\section{Discussion}

In this study we showed that DEPTOR, a protein overexpressed in MM ${ }^{13}$, maintains PC differentiation. We also found that miR-135b and miR-642a, downregulated in the disease, modulate DEPTOR levels in MM cells.

Initial studies of PC maturation reported that the final step of PC differentiation was irreversible 9,31-33. However, recent studies have revealed that alterations in IRE1, XBP1, FOXP1, PAX5 or BCL6/MTA3 may reprogram PCs to previous stages of differentiation 34-37. In keeping with these reports, we found that DEPTOR knockdown changed the transcriptional program associated with PC differentiation through the upregulation of PAX5 and BCL6, which maintain the B cell program, and downregulation of IRF4, a factor that favors PC differentiation 12. The main function of PC is the production of immunoglobulins at high rate, and for this to happen PCs must display a highly specialized morphology with expanded cytoplasm and a more sophisticated ER network compared with B cells ${ }^{38}$. Our results demonstrated a clear loss of cell size and ER mass in both H929 and MM1S as a consequence of DEPTOR downregulation. Taking these results together, we propose that DEPTOR maintains the state of PCs, and its deficiency in PCs results in PC dedifferentiation. Accordingly, DEPTOR levels increased during the differentiation of human PCs from B cells. Peterson et al showed that DEPTOR overexpression is necessary to maintain PI3K and AKT activation and that a reduction in DEPTOR levels leads to apoptosis 13.

Here, we found that DEPTOR inhibition, at the times and under the conditions assayed, did not affect cell survival, but reverted the commitment of PCs. A few earlier studies have found a connection between DEPTOR levels, mTORC1/mTORC2 activities and cell differentiation. It has been reported that DEPTOR maintains stem cell pluripotency by limiting mTOR activity in undifferentiated embryonic stem cells (ESCs) ${ }^{39}$, whereas differentiation of mouse ESCs is associated with decreased DEPTOR levels. In T cells, it has been demonstrated that mTOR drives T-cell differentiation and function ${ }^{40,41}$. In B cells one study has analyzed the consequences of B cell-specific loss of the mTOR negative regulator TSC1 ${ }^{42}$. The authors showed that deletion of TSC1 in murine B cells and subsequent TORC1 activation led to impairment of B-cell maturation. This work appears to be in agreement with our findings, in the sense that B cells would need an mTORC1 inhibitor to promote PC differentiation. However, we unexpectedly found that mTORC1/mTORC2 activities were not modified by DEPTOR silencing under our experimental conditions. The independence of mTORC1 activity was corroborated by the addition of rapamycin, an mTORC1 inhibitor that did not revert the PC dedifferentiation induced by DEPTOR knockdown. We hypothesize that 
DEPTOR may regulate B cell differentiation through mTOR-independent pathways. Their molecular connections with PC differentiation need to be elucidated.

DEPTOR has been found to be overexpressed in many tumor types, including breast cancer, prostate cancer, chronic myeloid leukemia, lung cancer and MM ${ }^{16}$. In the latter, DEPTOR overexpression was associated with cyclin D1/D3 upregulation and especially with the presence of $M A F / M A F B$ translocations ${ }^{13}$. The involvement of miRNAs in the pathogenesis and biology of myeloma has been suggested by several groups $27,43,44$. Here, we found that DEPTOR expression is also controlled by two miRNAs, miR-135b and miR-642a, both of which are downregulated in several MM patients ${ }^{27}$. Using luciferase reporter assays and gain-of-function experiments, we showed that transfection of miR-135b and miR-642a decreased DEPTOR levels in myeloma cells. Moreover, inhibition of miR-135b and miR642a in two MMCLs exhibiting high levels of expression of both miRNAs and a low level of expression of DEPTOR, despite displaying MAF or cyclinD1 upregulation, resulted in DEPTOR overexpression. We observed that DEPTOR downregulation induced by miR-135b and miR-642a ectopic expression also reverted the transcriptional program of the myeloma cells and reduced cell size and ER mass, similarly to the results obtained from DEPTOR silencing by siRNA. These findings emphasize the role that these miRNAs play in regulating DEPTOR expression.

It was of particular note that DEPTOR protein levels in myeloma cells varied from patient to patient, and that its upregulation was clearly associated with longer PFS. Interestingly, high levels of DEPTOR expression have previously been associated with the prediction of response to thalidomide in $M M{ }^{28}$. This observation is consistent with the fact that the patients included in our study received a treatment regimen that contained lenalidomide, a thalidomide-like drug. On the other hand, we have shown that DEPTOR induces PC maturation, and it has been reported that the maturation of myeloma cells is associated with sensitivity to anti-myeloma agents $30,34,45-47$, including lenalidomide ${ }^{45}$. In fact, plasma cell maturity seems to be an indicator of good prognosis in MM 47.

Overall, our results show that high levels of DEPTOR result in more mature myeloma cells that would be more sensitive to therapeutic agents. They suggest the merit of further investigations to test the potential of DEPTOR levels as an indicator of maturation and as a predictive biomarker of sensitivity to anti-myeloma therapy. 


\section{References}

1. Kyle RA and Rajkumar SV. Multiple myeloma. N Engl J Med. 2004;351(18):1860-1873.

2. Somasundaram R, Prasad MA, Ungerback J, Sigvardsson M. Transcription factor networks in B-cell differentiation link development to acute lymphoid leukemia. Blood. 2015;126(2):144-152.

3. Familiades J, Bousquet M, Lafage-Pochitaloff M, et al. PAX5 mutations occur frequently in adult B-cell progenitor acute lymphoblastic leukemia and PAX5 haploinsufficiency is associated with BCR-ABL1 and TCF3-PBX1 fusion genes: a GRAALL study. Leukemia. 2009;23(11):1989-1998.

4. Traverse-Glehen A, Verney A, Baseggio L, et al. Analysis of BCL-6, CD95, PIM1, RHO/TTF and PAX5 mutations in splenic and nodal marginal zone B-cell lymphomas suggests a particular B-cell origin. Leukemia. 2007;21(8):1821-1824.

5. Kirk SJ, Cliff JM, Thomas JA, Ward TH. Biogenesis of secretory organelles during B cell differentiation. J Leukoc Biol. 2010;87(2):245-255.

6. Kazi AA, Hong-Brown L, Lang SM, Lang CH. Deptor knockdown enhances mTOR Activity and protein synthesis in myocytes and ameliorates disuse muscle atrophy. Mol Med. 2011;17(9-10):925-936.

7. Jourdan M, Caraux A, Caron G, et al. Characterization of a transitional preplasmablast population in the process of human B cell to plasma cell differentiation. J Immunol. 2011;187(8):3931-3941.

8. Gass JN, Gunn KE, Sriburi R, Brewer JW. Stressed-out B cells? Plasma-cell differentiation and the unfolded protein response. Trends Immunol. 2004;25(1):17-24.

9. Nutt SL, Taubenheim N, Hasbold J, Corcoran LM, Hodgkin PD. The genetic network controlling plasma cell differentiation. Semin Immunol. 2011;23(5):341-349.

10. Calame KL, Lin KI, Tunyaplin C. Regulatory mechanisms that determine the development and function of plasma cells. Annu Rev Immunol. 2003;21:205-230.

11. Shapiro-Shelef M and Calame K. Regulation of plasma-cell development. Nat Rev Immunol. 2005;5(3):230-242. 
12. Nutt SL, Hodgkin PD, Tarlinton DM, Corcoran LM. The generation of antibody-secreting plasma cells. Nat Rev Immunol. 2015;15(3):160-171.

13. Peterson TR, Laplante M, Thoreen CC, et al. DEPTOR is an mTOR inhibitor frequently overexpressed in multiple myeloma cells and required for their survival. Cell. 2009;137(5):873-886.

14. Gutierrez NC, Ocio EM, de Las Rivas J, et al. Gene expression profiling of B lymphocytes and plasma cells from Waldenstrom's macroglobulinemia: comparison with expression patterns of the same cell counterparts from chronic lymphocytic leukemia, multiple myeloma and normal individuals. Leukemia. 2007;21(3):541-549.

15. Catena V and Fanciulli M. Deptor: not only a mTOR inhibitor. J Exp Clin Cancer Res. 2017;36(1):12-016-0484-y.

16. Wang Z, Zhong J, Inuzuka $\mathrm{H}$, et al. An evolving role for DEPTOR in tumor development and progression. Neoplasia. 2012;14(5):368-375.

17. Carrasco DR, Tonon G, Huang Y, et al. High-resolution genomic profiles define distinct clinico-pathogenetic subgroups of multiple myeloma patients. Cancer Cell. 2006;9(4):313325.

18. Misiewicz-Krzeminska I, Sarasquete ME, Quwaider D, et al. Restoration of microRNA214 expression reduces growth of myeloma cells through positive regulation of P53 and inhibition of DNA replication. Haematologica. 2013;98(4):640-648.

19. Budczies J, Klauschen F, Sinn BV, et al. Cutoff Finder: a comprehensive and straightforward Web application enabling rapid biomarker cutoff optimization. PLoS One. 2012;7(12):e51862.

20. Klein U, Casola S, Cattoretti G, et al. Transcription factor IRF4 controls plasma cell differentiation and class-switch recombination. Nat Immunol. 2006;7(7):773-782.

21. Hurt EM, Wiestner A, Rosenwald A, et al. Overexpression of c-maf is a frequent oncogenic event in multiple myeloma that promotes proliferation and pathological interactions with bone marrow stroma. Cancer Cell. 2004;5(2):191-199.

22. Eychene A, Rocques N, Pouponnot C. A new MAFia in cancer. Nat Rev Cancer. 2008;8(9):683-693. 
23. Gabrea A, Bergsagel PL, Chesi M, Shou Y, Kuehl WM. Insertion of excised IgH switch sequences causes overexpression of cyclin D1 in a myeloma tumor cell. Mol Cell. 1999;3(1):119-123.

24. Herath NI, Rocques N, Garancher A, Eychene A, Pouponnot C. GSK3-mediated MAF phosphorylation in multiple myeloma as a potential therapeutic target. Blood Cancer J. 2014;4:e175.

25. Lombardi L, Poretti G, Mattioli M, et al. Molecular characterization of human multiple myeloma cell lines by integrative genomics: insights into the biology of the disease. Genes Chromosomes Cancer. 2007;46(3):226-238.

26. Chang H, Qi Q, Xu W, Patterson B. c-Maf nuclear oncoprotein is frequently expressed in multiple myeloma. Leukemia. 2007;21(7):1572-1574.

27. Gutierrez NC, Sarasquete ME, Misiewicz-Krzeminska I, et al. Deregulation of microRNA expression in the different genetic subtypes of multiple myeloma and correlation with gene expression profiling. Leukemia. 2010;24(3):629-637.

28. Boyd KD, Walker BA, Wardell CP, et al. High expression levels of the mammalian target of rapamycin inhibitor DEPTOR are predictive of response to thalidomide in myeloma. Leuk Lymphoma. 2010;51(11):2126-2129.

29. Todoerti K, Agnelli L, Fabris S, et al. Transcriptional characterization of a prospective series of primary plasma cell leukemia revealed signatures associated with tumor progression and poorer outcome. Clin Cancer Res. 2013;19(12):3247-3258.

30. Goasguen JE, Zandecki M, Mathiot C, et al. Mature plasma cells as indicator of better prognosis in multiple myeloma. New methodology for the assessment of plasma cell morphology. Leuk Res. 1999;23(12):1133-1140.

31. Shaffer AL, Lin KI, Kuo TC, et al. Blimp-1 orchestrates plasma cell differentiation by extinguishing the mature B cell gene expression program. Immunity. 2002;17(1):51-62.

32. Ferrell JE,Jr. Self-perpetuating states in signal transduction: positive feedback, doublenegative feedback and bistability. Curr Opin Cell Biol. 2002;14(2):140-148.

33. Monod J and Jacob F. Teleonomic mechanisms in cellular metabolism, growth, and differentiation. Cold Spring Harb Symp Quant Biol. 1961;26:389-401. 
34. Leung-Hagesteijn C, Erdmann N, Cheung G, et al. Xbp1s-negative tumor B cells and preplasmablasts mediate therapeutic proteasome inhibitor resistance in multiple myeloma. Cancer Cell. 2013;24(3):289-304.

35. van Keimpema M, Gruneberg LJ, Mokry M, et al. The forkhead transcription factor FOXP1 represses human plasma cell differentiation. Blood. 2015;126(18):2098-2109.

36. Fujita N, Jaye DL, Geigerman C, et al. MTA3 and the Mi-2/NuRD complex regulate cell fate during B lymphocyte differentiation. Cell. 2004;119(1):75-86.

37. Mikkola I, Heavey B, Horcher M, Busslinger M. Reversion of B cell commitment upon loss of Pax5 expression. Science. 2002;297(5578):110-113.

38. Bettigole SE and Glimcher LH. Endoplasmic reticulum stress in immunity. Annu Rev Immunol. 2015;33:107-138.

39. Agrawal P, Reynolds J, Chew S, Lamba DA, Hughes RE. DEPTOR is a stemness factor that regulates pluripotency of embryonic stem cells. J Biol Chem. 2014;289(46):31818-31826.

40. Powell JD and Delgoffe GM. The mammalian target of rapamycin: linking T cell differentiation, function, and metabolism. Immunity. 2010;33(3):301-311.

41. Chapman NM and Chi H. mTOR Links Environmental Signals to T Cell Fate Decisions. Front Immunol. 2015;5:686.

42. Benhamron $S$ and Tirosh B. Direct activation of mTOR in B lymphocytes confers impairment in B-cell maturation andloss of marginal zone B cells. Eur J Immunol. 2011;41(8):2390-2396.

43. Pichiorri F, Suh SS, Ladetto M, et al. MicroRNAs regulate critical genes associated with multiple myeloma pathogenesis. Proc Natl Acad Sci U S A. 2008;105(35):12885-12890.

44. Lionetti M, Biasiolo M, Agnelli L, et al. Identification of microRNA expression patterns and definition of a microRNA/mRNA regulatory network in distinct molecular groups of multiple myeloma. Blood. 2009;114(25):e20-6.

45. Kawano Y, Fujiwara S, Wada N, et al. Multiple myeloma cells expressing low levels of CD138 have an immature phenotype and reduced sensitivity to lenalidomide. Int J Oncol. 2012;41(3):876-884. 
46. Kuroda Y, Sakai A, Okikawa Y, et al. The maturation of myeloma cells correlates with sensitivity to chemotherapeutic agents. Int J Hematol. 2005;81(4):335-341.

47. Iriyama N, Miura K, Hatta Y, et al. Plasma cell maturity as a predictor of prognosis in multiple myeloma. Med Oncol. 2016;33(8):87-016-0803-3. Epub 2016 Jul 6. 


\section{Supplementary materials}

Supplementary Tables:

Table S1. List of oligonucleotide sequences used for 3'UTR luciferase constructs.

\begin{tabular}{|c|c|}
\hline \multicolumn{2}{|c|}{ Sequence $\left(5^{\prime}-3^{\prime}\right)$} \\
\hline \multicolumn{2}{|c|}{ DEPTOR-miR-135b WT } \\
\hline Forward & AAACAAGCATTCAAGTGCTTAAAAGCCATAAAAAATGACTTCTTAATTT \\
\hline Reverse & CTAGAAATTAAGAAGTCATTTTTTATGGCTTTTAAGCACTTGAATGCTTGTTT \\
\hline \multicolumn{2}{|c|}{ DEPTOR-miR-135b MUT } \\
\hline Forward & AAACAAGCATTCAAGTGCTTAAAAGCTGCGAAAAATGACTTCTTAATTT \\
\hline Reverse & CTAGAAATTAAGAAGTCATTTTTCGCAGCTTTTAAGCACTTGAATGCTTGTTT \\
\hline \multicolumn{2}{|c|}{ DEPTOR-miR-642a WT } \\
\hline Forward & AAACTCCAGTGGCCTGTGGGTGAGGGAAGCCAGAATGACACAAAT \\
\hline Reverse & CTAGATTTGTGTCATTCTGGCTTCCCTCACCCACAGGCCACTGGAGTTT \\
\hline \multicolumn{2}{|c|}{ DEPTOR-miR-642a MUT } \\
\hline Forward & AAACTCCAGTGGCCTGTGGGTAGAAAGGACCAGAATGACACAAAT \\
\hline Reverse & CTAGATTTGTGTCATTCTGGTCCTTTCTACCCACAGGCCACTGGAGTTT \\
\hline
\end{tabular}




\section{Supplementary Figures}

A

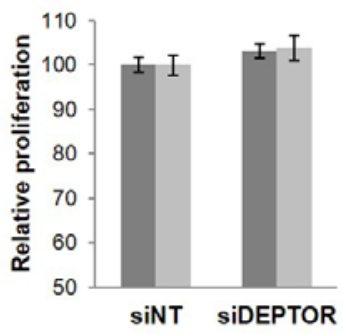

B

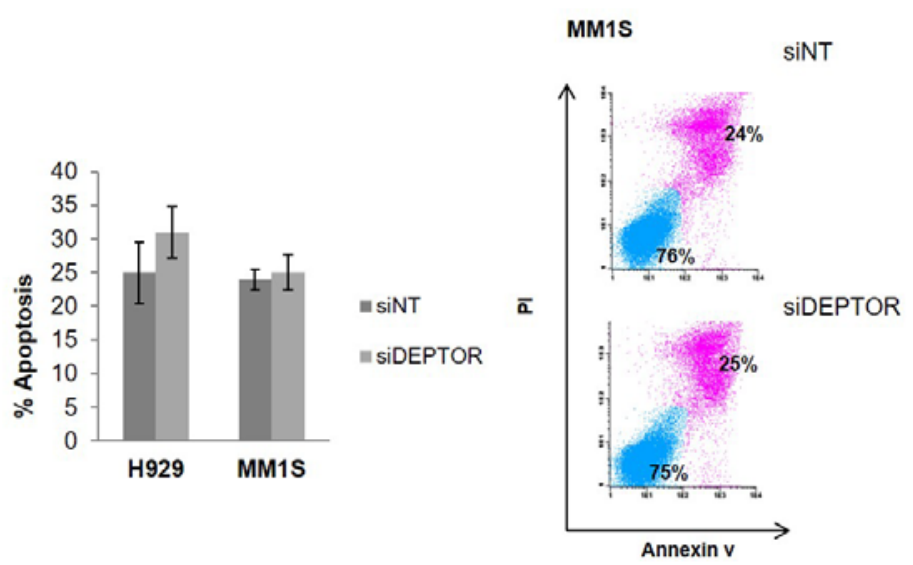

Figure S1. DEPTOR knockdown did not alter cell viability. A Proliferation of myeloma cells $48 \mathrm{~h}$ after transfection with DEPTOR siRNA. Data are expressed as means of three independent experiments \pm SD. Proliferation of cells transfected with siNT was taken as $100 \%$ and values obtained in DEPTOR-silenced cells were normalized accordingly. B Percentage of apoptosis after DEPTOR knockdown in H929 and MM1S. Right panel shows representative dot plots.

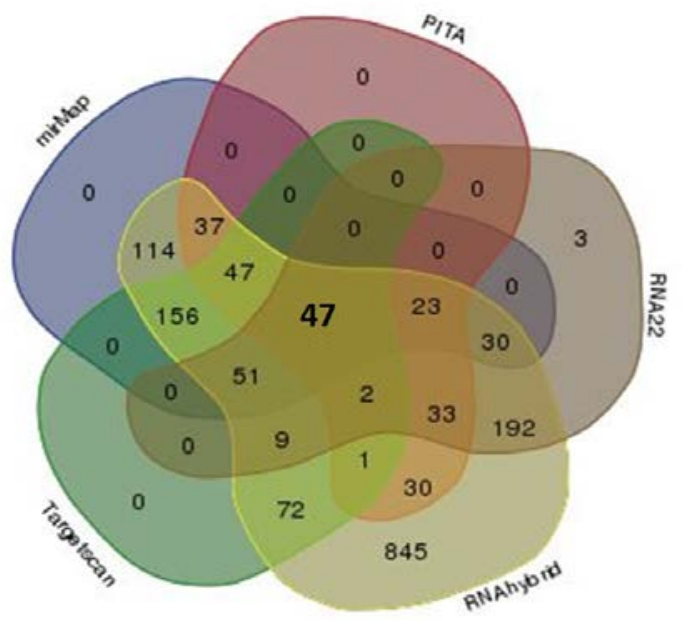

miRNA-DEPTOR interaction prediction algorithms

Figure S2. Bioinformatic identification of miRNAs that regulate DEPTOR expression. Venn diagram showing numbers of miRNAs predicted to target DEPTOR by the indicated 5 databases. 
A

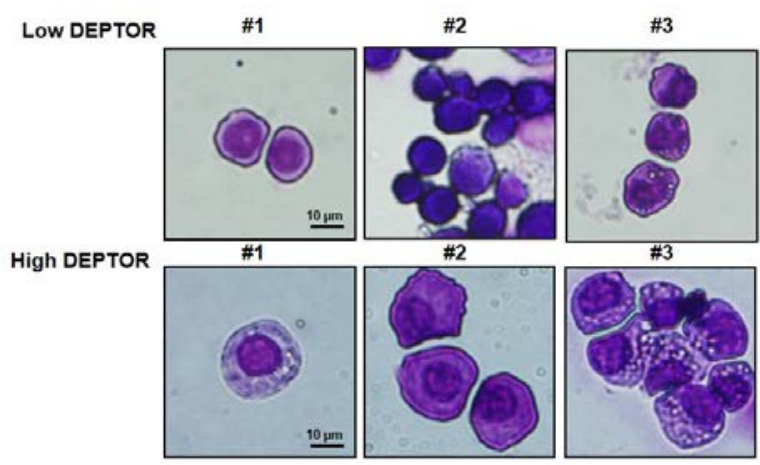

B

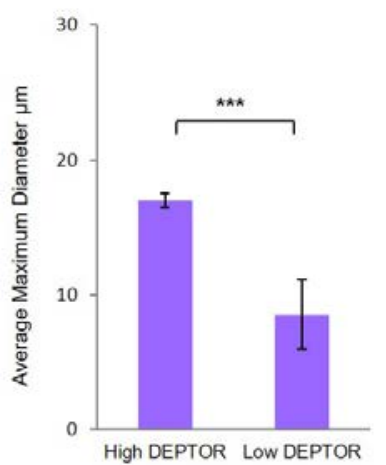

Figure S3. Cell morphology and size in MM patients with different DEPTOR levels. A Giemsa stain of 3 MM patients. B Average maximum diameter of MM cells measured from patients harboring high $(n=3)$ and low $(n=3)$ DEPTOR levels. At least 50 cells per experiment were counted. $\left(^{*} p<0.05\right.$, ** $\left.p<0.01,{ }^{* * *} p<0.001\right)$. 



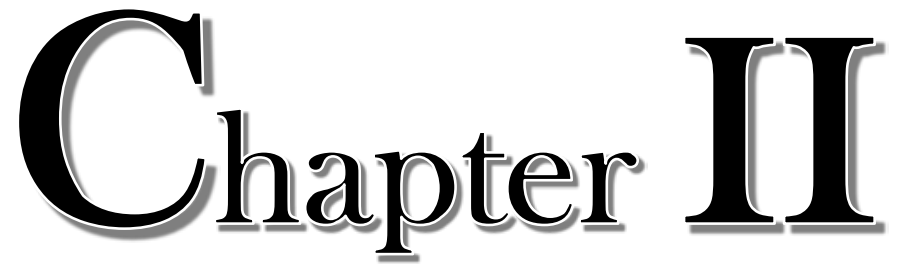

\section{MiR-124 and miR-506 overexpression promote plasma cell dedifferentiation through the regulation of IRE1}

Dalia Quwaider ${ }^{1,2.3}$, Luis A. Corchete ${ }^{1.2 .3}$, Ana B. Herrero ${ }^{1,27}$, and Norma C. Gutiérrez ${ }^{1.147}$

$\dagger$ Equal contributors

'Institute of Biomedical Research of Salamanca (IBSAL), Salamanca, Spain; ${ }^{2}$ Cancer Research Center-IBMCC (USAL-CSIC), Salamanca, Spain; ${ }^{3}$ Haematology Department, University Hospital of Salamanca, Spain; ${ }^{4}$ Center for Biomedical Research in Network of Cancer (CIBERONC), Salamanca, Spain. 



\section{Abstract}

B cell maturation is a serial process of multiple steps controlled by several relevant pathways, transcription factors, and miRNAs. Here, we investigate the role of IRE1 in the terminal differentiation of myeloma cells. IRE1 knockdown in H929 and MM1S cell lines induced dedifferentiation of myeloma cells, as demonstrated by the upregulation of PAX5 and BCL6. In addition, we show that miR-124 and miR-506 regulate IRE1 expression in MM using luciferase reporter assays and gain-of-function experiments. The ectopic expression of miR-124 and miR-506 in myeloma cell lines substantially reduced IRE1 protein levels, and caused dedifferentiation of myeloma cells, as shown by the downreglulation of IRF4 and $\operatorname{IgK}$, and the reduction in cell size and endoplasmic reticulum mass. Our results demonstrate that IRE1 expression is required to maintain myeloma cell differentiation. 



\section{Introduction}

Endoplasmic reticulum (ER) plays a pivotal role in the synthesis, folding, and maturation of up to $30 \%$ of proteins in the cell ${ }^{1}$. Changes in environmental or physiological demand lead to rapid increases in the protein-folding rate in the ER. The development of an immune response requires synthesis of a broad range of proteins, including inflammatory mediators, costimulatory molecules and antibodies. However, if the protein folding capacity of the cell is not synchronized with the protein folding demand, misfolded proteins are accumulated in the lumen of the organelle causing ER stress ${ }^{2}$.

ER stress in turn activates the unfolded protein response (UPR), an adaptive cellular response controlled by three ER-resident sensors: PERK, ATF6, and IRE1. Once activated, these proteins collaborate in order to maintain ER homeostasis and ensuring cell survival ${ }^{2}$. IRE1 is the most conserved transducer of the UPR and the most studied in the immune system ${ }^{3,4}$. Upon UPR activation, IRE1 splices, at two specific sites, a 26-nucleotide sequence from the un-spliced XBP1 mRNA (XBP1u), which induces a translational frameshift that results in the expression of a fully functional transcription factor XBP1s (the spliced form) that is essential for PC commitment 5,6. XBP1 also activates the expression of a variety of UPR target genes 7 . Accordingly, UPR is indispensable for ER expansion and protein secretion, and therefore is needed for development, differentiation, and the precise function of highly secretory cells, such as PCs ${ }^{3,8,9}$. IRE1-XBP1 branch is essential during PC differentiation, especially for the transition from activated BC into PC stage, where the activation of UPR is necessary to accommodate the increased production and secretion of Igs, in the course of ER expansion 10-12. Moreover, the IRE1 conditional knockout mice have served to demonstrate that IRE1 is also required for efficient antibody production in PCs 10.

The regulation of UPR by microRNAs has been reported in different cell types ${ }^{13}$. MiR-30a, miR181a and miR-199a-5p, which are downregulated in prostate, colon and bladder tumors, have been demonstrated to target BiP (GRP78), the major ER chaperone and signaling regulator ${ }^{14}$. In addition, post-transcriptional regulation of IRE1 by miRNAs has been shown in hepatoma cells ${ }^{15}$. Meanwhile the post-transcriptional regulation of IRE1 by miRNAs has not been studied in MM.

Reanalyzing microarray expression data previously generated by our group, we found that the mRNA-encoding IRE1 was overexpressed in NPCs and myeloma cells compared with normal B lymphocytes (NBLs) ${ }^{16}$, in agreement with previous studies in which this endoribonuclease was shown to contribute to PC differentiation 10,17,18. 
Accordingly, we hypothesized that the downregulation of IRE1 in MM cells could revert the maturation state of these cells, which had not been previously reported. Studies using RNAi technology to knockdown IRE1 expression or the use of specific inhibitors of IRE1 RNase activity demonstrated that IRE1 suppression induced cell death in myeloma cells 19-21. On the other hand, during the development of the present work, Leung-Hagesteijn published that the knockdown of XBP1 or IRE1 by shRNA in myeloma cells did not induce cell death but resulted in modest reversal of PC differentiation.

Here, we report for the first time that miR-124 and miR-506 regulate IRE1 expression in multiple myeloma and IRE1 expression is required to maintain myeloma cells at the terminal stage of differentiation. 


\section{Materials and methods}

\section{Cell lines and primary samples}

The human multiple myeloma cell lines (MMCL), NCI-H929, and MM1S, were acquired from the ATCC (American Type Culture Collection). JJN3 and HEK923 lines were obtained from the "Deutsche Sammlung von Mikroorganismen und Zellkulturen" (DSMZ). Cell line identity was confirmed periodically by STR analysis with thePowerPlex 16 HS system kit (www.promega.com) and on-line STR matching analysis (www.dsmz.de/fp/cgi-bin/str.html). Cell lines were cultured in RPMI 1640 medium supplemented with $10 \%$ fetal bovine serum and antibiotics (Gibco Life Technologies, Grand Island, NY, USA). Bone marrow (BM) samples from ten healthy donors were sorted by a FACSAria equipment into four BC populations: immature B cells (CD34-, CD19+, CD10+, CD38++), naive B cells (CD19+, CD27-,CD10-), memory B cells (CD19+, CD138-, CD27+, CD38+), and plasma cells(CD38+++, CD138+, CD45low). Monoclonal antibodies were purchased as follows: anti-CD45-FITC (clone D3/9) and anti-CD19-PECy7 (clone A3-B1) from Immunostep (Salamanca, Spain); anti-CD38-PerCP-Cy ${ }^{\mathrm{TM}} 5.5$ (cloneHIT2), anti-CD34-APC (clone 8G12), and anti-CD27-BV421 (clone M-T271) from BD Biosciences (San Jose,CA, USA); anti- CD138-Pacific OrangeTM (clone B-A38) from Exbio Praha (Vestec, Czech Republic); and anti-CD10-PE (clone ALB1) from Beckman Coulter (Pasadena,CA, USA).

\section{RNA extraction and quantitative real-time PCR analysis}

RNA was extracted from the cell lines using an RNeasymini kit (Qiagen, Valencia, USA) according to the standard protocol. RNA integrity was assessed using an Agilent 2100 Bioanalyzer (Agilent Technologies, Santa Clara, CA, USA). Total RNA $(1 \mu \mathrm{g})$ was reverse transcribed to complementary DNA (cDNA) using High-Capacity cDNA Reverse Transcription Kits (Ap-plied Biosystems, Foster City, CA, USA). Expression of target genes was assessed using TaqMan qRT-PCR assays (Applied Biosystems). Relative gene expression was calculated by the $2^{-\triangle C t}$ method using GAPDH as the endogenous control for normalization. To detect mature miR124 and miR-506 expression levels, TaqMan quantitative real-time polymerase chain reaction (qRT-PCR) microRNA assay (AppliedBiosystems) was performed. The relative levels of expression of mature miR-124 and miR-506 normalized with respect to the RNU43 endogenous control were determined by the $2^{-\Delta C t}$ method. Each measurement was performed in triplicate. 


\section{Transfections}

Cell lines were transfected using the nucleofector II sys-tem (Lonza, Allendale, NJ, USA) with the following programs: C-16 for H929 and G-16 for MM1S. Cells were transfected with premiR ${ }^{\mathrm{TM}}$ miRNA precursors pre-miR-124, pre-miR-506, and pre-miR ${ }^{\mathrm{TM} m i R N A}$ negative nontargeting control \#1 (Ambion, Austin, TX, USA) at $25 \mathrm{nM}$ concentration.

\section{Cell proliferation assay}

Cell viability was evaluated with the CellTiter-Glo® luminescent cell viability assay based on the amount of ATP present (Promega), in accordance with the manufacturer'sprotocol.

\section{Apoptosis assay}

Caspase $3 / 7,8$ and 9 activity was evaluated by Caspase-Glo $\AA 3 / 7$, Caspase-Glo $₫ 8$ and CaspaseGlo®9 Assays (Promega), respectively, according to the manufacturer's protocol. In the assays, 20,000 cells/well were incubated with the reagents at room temperature for $1 \mathrm{~h}$ in the case of caspase $3 / 7$, and $2 \mathrm{~h}$ for caspases 8 and 9 . Light intensity was measured in each well using a Tekan Infinite $® F 500$ microplate reader.

\section{Immunophenotyping}

MM cell lines were immunophenotyped on a FACS-Canto II cytometer (Beckton Dickinson Biosciences) using the following monoclonal antibody: CD38-APC-H7 (BD Biosciences). Data analysis was performed using the Infinicyt software (Cytognos S.L.). A minimum of $10^{5}$ events were stored.

\section{Luciferase reporter assay}

The double-stranded oligonucleotides corresponding to the wild-type or mutant miR-124, miR-506, miR-488, and miR-326 binding sites in the 3'-untranslated region (3'UTR) of IRE1 were synthesized (Supplementary materials: Table S1) (Sigma-Al-drich, St Louis, MO, USA) and ligated between the PmeI and Xbal restriction sites of the pmirGLO vector (Promega, Madison, WI, USA). Oligonucleotide sequences are detailed in (Supplementary Table S1). Luciferase assays in HEK293 cells were performed as previously described 22 . 


\section{Western blot}

Whole cell lysates were collected using RIPA buffer (Sigma-Aldrich) containing protease inhibitors (Complete Protease Inhibitor Cocktail Tablets, Roche) and phosphatase inhibitors (Phosphatase Inhibitor Cocktail A and B, Santa Cruz Biotechnology). Protein concentration was measured using the Bradford assay (BioRad). Protein samples ( $30 \mu \mathrm{g} / \mathrm{lane}$ ) were subjected to sodium dodecyl sulphate-polyacrylamide gel electrophoresis (SDS-PAGE) and transferred to $0.45 \mathrm{~nm}$ polyvinylidene fluoride (PVDF) membranes (BioRad). The primary antibodies used for immunoblotting were: anti-IRE1, anti-IRF4, anti-Ig kappa light chain from Santa Cruz Biotech (Delaware, CA, USA). Anti- $\beta$-actin (Sigma-Aldrich) was used as an internal control for protein loading. The membranes were then washed and incubated with the secondary horseradish peroxidase-linked antibodies: anti-mouse IgG or anti-rabbit IgG antibodies (PierceNet) (1:10000), or anti-goat IgG (Santa Cruz Biotech) (1:10000). Chemiluminescence was detected using the Amersham ECL Plus ${ }^{\mathrm{TM}}$ WesternBlotting Detection Reagent (GE Healthcare).

\section{Immunofluorescence staining}

Cells were collected $72 \mathrm{~h}$ post-transfection, washed with PBS, and stained for $30 \mathrm{~min}$ with $1 \mu \mathrm{M}$

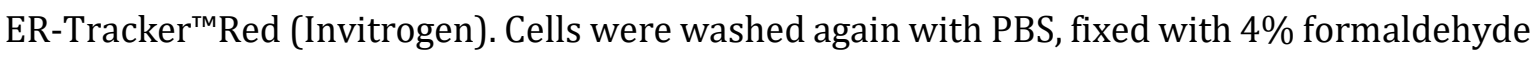
for $5 \mathrm{~min}$ at room temperature, placed on glass slides coated with poly-L-lysine, and stained for 1 min with DAPI II. The slides were then mounted using VECTASHIELD Mounting Medium (Vector Laboratories, Burlingame, CA, USA). Images were collected under a Zeiss confocal microscope equipped with 636/1.4 Oil Plan-APOCRHOMAT DIC.

\section{IRE1 gene silencing by short hairpin RNA}

Silencing of IRE1 was performed using short hairpin RNA (shRNA) sequences cloned into the lentiviral vector pLKO.1. Five plasmids containing shRNAs targeting IRE1 were acquired from Dharmacon. The efficiency of these plasmids to downregulate IRE1 was analyzed by measuring the levels of IRE1 protein by Western blot after $72 \mathrm{~h}$ of transfection of HEK293T cells. The plasmid that induced the strongest silencing effect was selected and used as transfer vector to construct lentiviral particles using the calcium phosphate protocol. HEK293T cells were plated in $10 \mathrm{~cm}$ plates at approximately $2 \times 10^{6}$ cells/plate. The following day, a plasmid mixture was prepared containing $10 \mu \mathrm{g}$ of the pLKO.1-IRE1 transfer plasmid or PLK0.1-TRC control, $7.5 \mu \mathrm{g}$ of PAX2 and $2.5 \mu \mathrm{g}$ pMD2-G, used as packaging plasmids. Sixty-four $\mu \mathrm{L}$ of $\mathrm{CaCl} 2 \mathrm{M}$ was added to this mixture and it was completed to $436 \mu \mathrm{L}$ with miliQ water. Then, $500 \mu \mathrm{L}$ of HBS was added dropwise onto the plasmid mixture with continuous bubbling. The mixture was left 5 minutes at room temperature and then was added to the plate containing HEK293T cells. After 20 hours of 
culture, the medium was changed and after 48 hours, the supernatant containing the generated viruses was collected and filtered using a $45 \mu \mathrm{m}$-filter syringe. Then, MM cells were transduced approximately $5 \times 10^{6}$ cells were infected with $10 \mathrm{~mL}$ of the viruscontaining supernatant and polybrene $(5 \mu \mathrm{g} / \mathrm{L})$. After 24 hours, the medium was changed and the effect of the transduction on IRE1 expression was analyzed by Western blot after 72 hours of culture. 


\section{Results}

\section{IRE1 is overexpressed in the last stage of B cell differentiation.}

In order to confirm the overexpression of IRE1 in PC compared to earlier stages of B cell differentiation previously observed using microarray analysis (GSE6691 at GE0 repository) ${ }^{16}$ (Figure 1A), we quantified IRE1 mRNA levels by qRT-PCR in four populations: immature, naïve, memory B cells and PCs isolated from BM samples obtained from healthy donors. IRE1 expression was found to be significantly higher in PCs than in all previous stages of differentiation. Next, we evaluated IRE1 expression in a panel of MM cell lines (Figure 1B). IRE1 mRNA was detected in all cell lines, particularly in MM1S and U266 cell lines, which exhibited the highest mRNA levels.

A

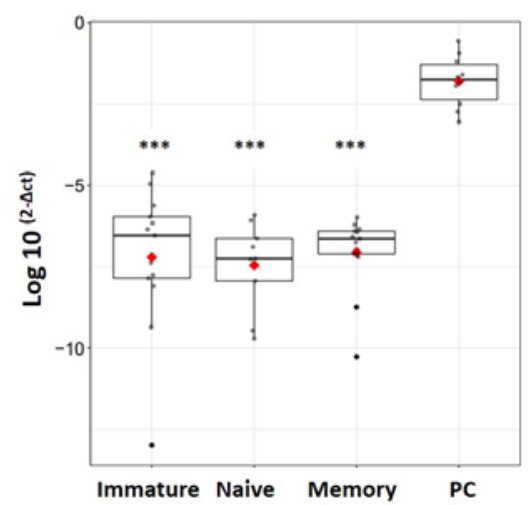

B

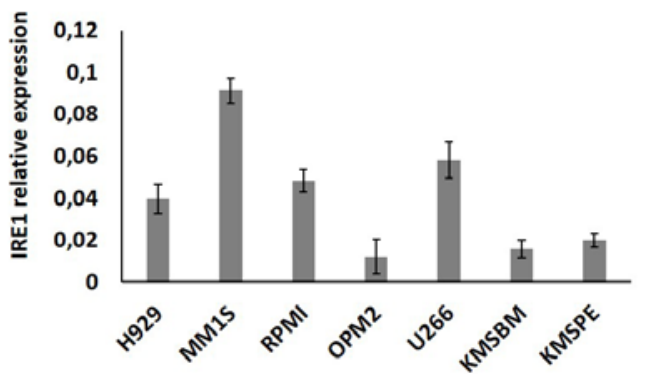

Figure 1. IRE1 expression in different B cell populations. A IRE1 expression detected by qRT-PCR in immature B cells, naive B cells, memory B cells and NPC. (*** $p<0.001)$. B Levels of IRE1 in MM cell lines measured by qRT-PCR. 


\section{IRE1 knockdown increases the expression of B cell lineage-specific activator proteins}

To analyze whether the presence of IRE1 in myeloma cells was necessary to maintain their state of differentiation we tried to silence its expression in MM cell lines using siRNA from different companies. However, none of them was able to decrease IRE1 mRNA levels, despite the fact that several siRNAs concentrations and times post-transfection were assayed (Figure $2 \mathrm{~A}$ and $2 \mathrm{~B}$ ). Therefore, we changed the approach and decided to use shRNA instead of siRNAs to downregulate IRE1 expression in MM.

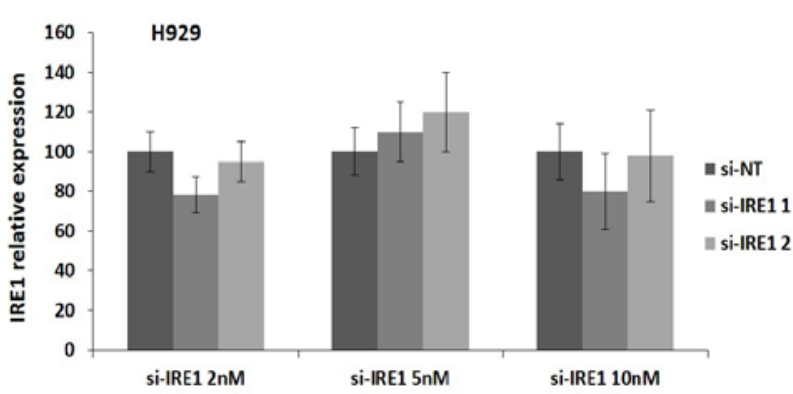

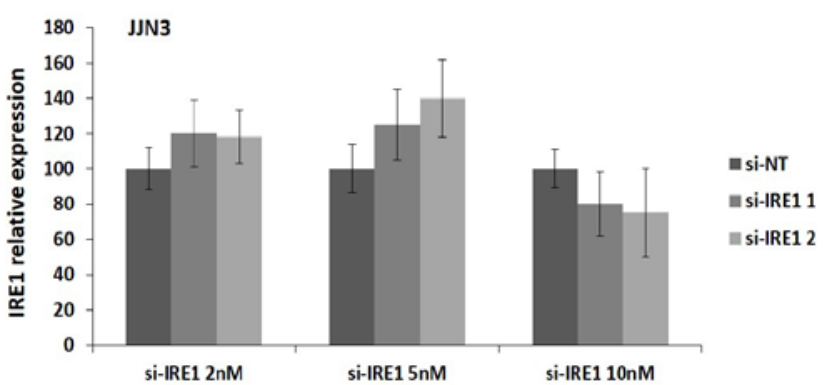

Figure 2. IRE1 knockdown in MM cell lines using siRNAs. A mRNA levels of IRE1 48h after IRE knockdown in H929 using two different type of siRNAs at different concentration. B mRNA levels of IRE1 48h after IRE knockdown in JJN3 using two different type of siRNAs at different concentration. 
H929 and MM1S were transduced with lentivirus carrying IRE1 shRNAs and infected cells were selected with puromycin. As can be observed in (Figure 3A), IRE1 protein levels were successfully reduced in both cell lines. Next, we analyzed the expression of PAX5 and BCL6, which encode B cell lineage-specific activator proteins present only at early stages of B cell differentiation ${ }^{18}$, and we found that both genes increased their expression in IRE1-knocked down cells (Figure 3B).

A

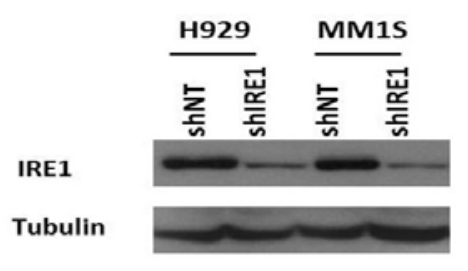

B
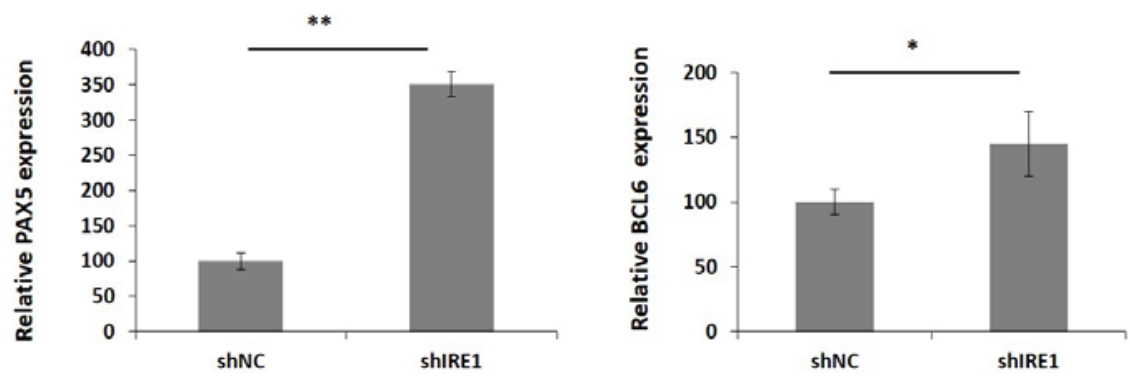

Figure 3. IRE1 knockdown in MM cell lines using shRNAs. A Western blot of IRE1 in H929 and MM1S cells. B mRNA levels of PAX5 and BCL6 in H929 quantified by qRT-PCR. 


\section{IRE1 is a direct target of miR-124, miR-506, miR-326, and miR-488}

To address the possibility that post-transcriptional regulation by microRNAs was involved in IRE1 expression, we looked for miRNA-IRE1 interactions in Targets scan database. According to the prediction algorithms, miR-124, miR-506, miR-326 and miR-488 have at least one putative site in the IRE1 3'UTR (Figure 4A). To determine whether IRE1 mRNA was a direct target of those miRNAs, we carried out luciferase reporter assays in HEK293 cells. The 3'UTR of IRE1 harboring the sequence complementary to the miR-124, miR-326, miR-506 or miR-488 seed sequence was cloned in a reporter plasmid vector referred to as wild-type (WT). In parallel, a 3'UTR IRE1 fragment containing mutant sequences (MUT) of the seed site of the four miRNAs was cloned in the same reporter plasmid. We found that luciferase activity of cells cotransfected with WT IRE1 3'UTR and miR-124, miR-326, miR-506 or miR-488 was significantly lower $(p<0.05$ or $p<0.01$ ) than that exhibited by cells transfected with the negative control (NC) miRNA. MiR-124 and miR-506 displayed a higher and more significant influence on luciferase activity. Luciferase activity of MUT constructs was not affected by miR-124, miR-326, miR-506 or miR-488 overexpression (Figure 4B), as expected.

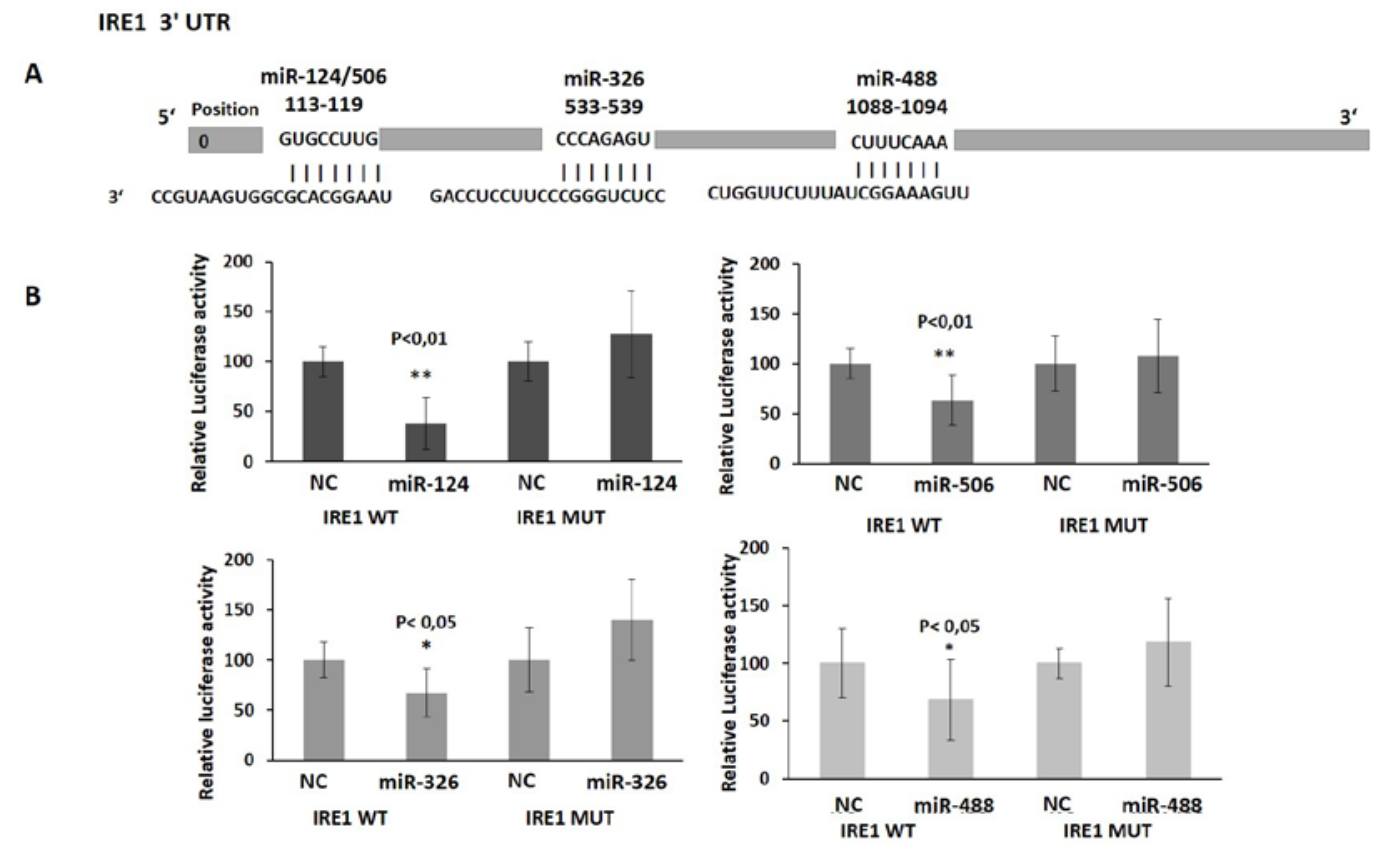

Figure 4. MiRNA target gene validation of IRE1 using a luciferase reporter gene assay. A Schematic diagram of the miR-124, miR-326, miR-488 and miR-506 predicted site on the IRE1 3'UTR. B Luciferase activity in HEK293 cells co-transfected with pre-miR-NC or pre-miR-124, miR-326, miR-506 or miR-488 and plasmid pmiR-Glo with the putative miR-124, miR-326, miR-506 or miR-488 binding site of IRE1 cloned downstream of the luciferase reporter gene. Luciferase activity was normalized using Renilla. All results are presented as the means \pm SD of three different experiments. $\left(* p<0.05,{ }^{* *} p<0.01\right)$. 


\section{MiR-124 and miR-506 regulate IRE1 expression in MM}

In order to evaluate the expression of miR-124, miR-506, miR-326 and miR-488 in MM cells, we measured their expression levels in a set of MM cell lines (JJN3, H929, MM1S, and RPMI 2886) using miRNA TaqMan qRT-PCR. miR-124 was moderately detected in all cell lines, while miR-488, miR-326 and miR-506 exhibited low expression in all cell lines with the exception of RPMI-8226, which exhibited higher level of miR-326 expression (Figure 5A). To test if these miRNAs regulate IRE1 expression in vivo, H929 was transfected with miR-124, miR-326, miR488 or miR-506 and negative control (NC) miRNA precursors. Clearly, lower levels of IRE1 protein expression were found in miR-124 and miR-506 transfected cells than in control cells (Figure 5B). Transfection with miR-326 or miR-488 did not affect IRE1 protein levels (data not shown).

A
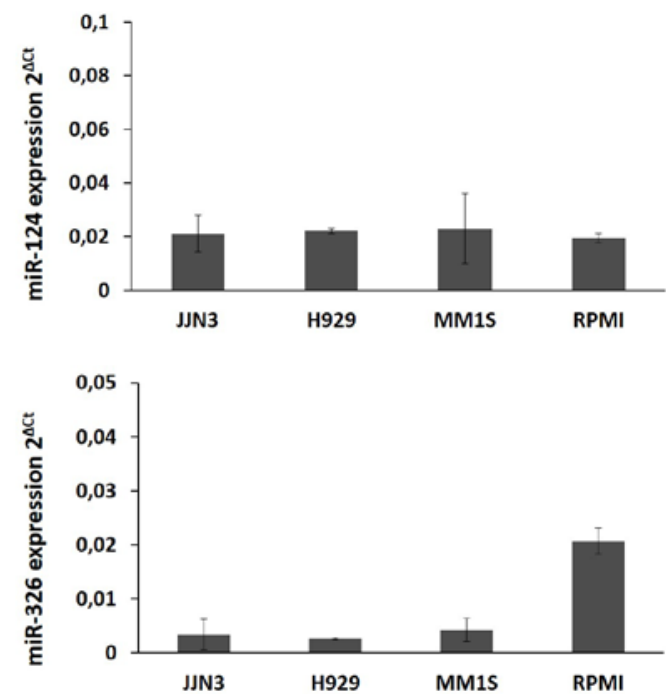
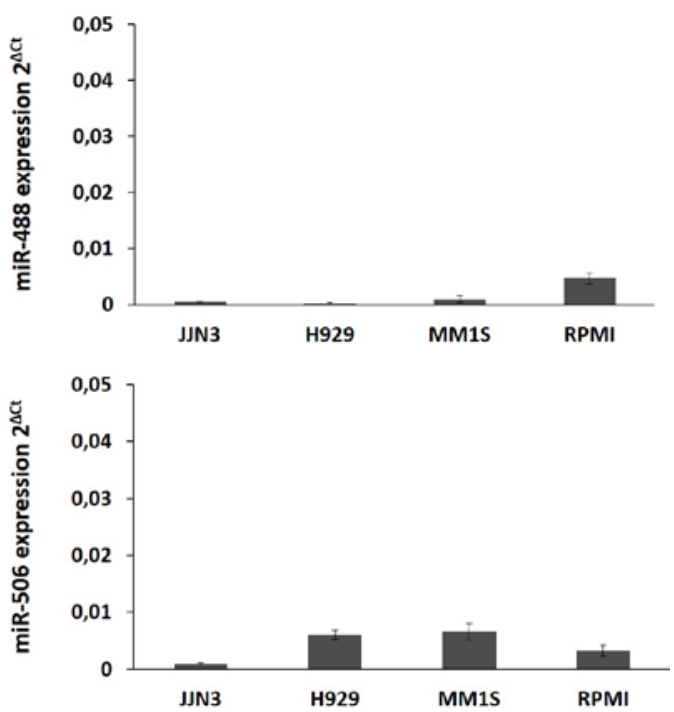

B

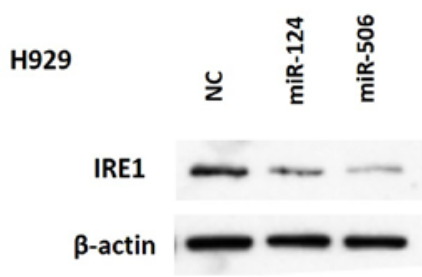

Figure 5. MiR-124 and miR-506 regulate IRE1 expression in MM. A miR-124, miR-326, miR-506 and miR-488 expression in MM cell lines measured by qRT-PCR. microRNA expression was normalized with respect to RNU43. B Western blot of IRE1 in H929 cells $72 \mathrm{~h}$ post-transfection with NC or miR-124/miR506. 


\section{Ectopic expression of miR-124 or miR-506 slightly decreases cell proliferation in MM}

Knockdown of IRE1 after transfection with miR-124 or miR-506 inhibited cell proliferation in H929 and MM1S, as assessed by measuring ATP levels (Figure 6A). To evaluate whether this effect was the result of apoptosis induction, caspase 3/7, 8 and 9 activities were analyzed in H929 and MM1S cell lines after transfection with miR-124 or miR-506 and NC miRNA precursors. We found that the activity of these caspases was increased only in H929 but not in MM1S (Figure 6B), indicating that induction of apoptosis seems to be cell line-dependent.

A
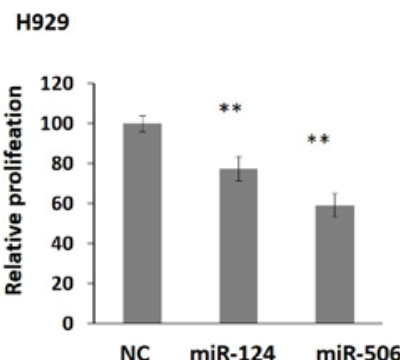

B

H929

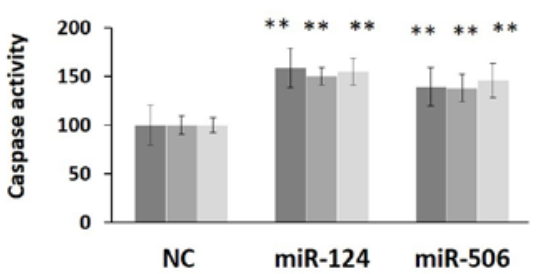

MM1S

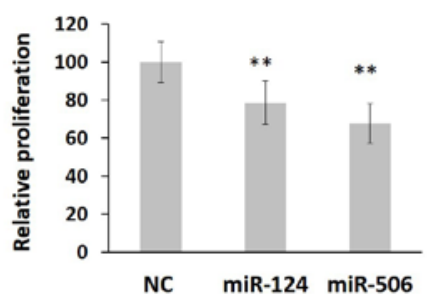

MM1S

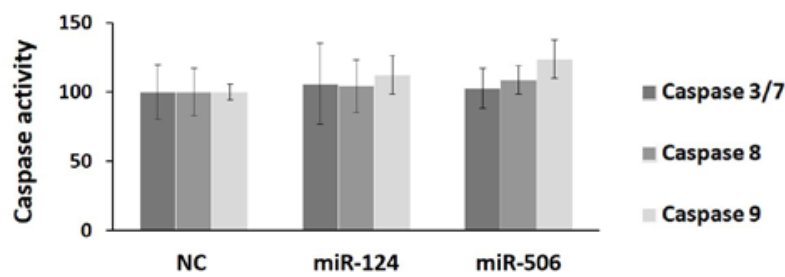

Figure 6. MiR-124 and miR-506 overexpression decreases MM cell proliferation. A Proliferation of myeloma cells $72 \mathrm{~h}$ after transfection with miR-124 or 506 or non-targeting control. Data are expressed as means of three independent experiments \pm SD. Proliferation of cells transfected with NC was taken as $100 \%$ and values obtained in miR-124 or 506 transfected cells were normalized accordingly. B Luminescent assays of caspases $3 / 7$, caspase 8 and caspase 9 . The results are presented as the mean \pm SD of three different experiments and considering the result after non-targeting control (NC) transfection as $100 \%$. ( $\left.{ }^{* *} p<0.01\right)$. 


\section{Upregulation of miR-124 and miR-506 results in myeloma cell dedifferentiation through the negative regulation of IRE1}

Next, we investigated whether the downregulation of IRE1 induced by the overexpression of miR-124 or miR-506 was able to affect the state of PC differentiation. To this end, we monitored different markers of PC maturation, such as IRF4, $\mathrm{k}$ light chain proteins and CD38. We found that the downregulation of IRE1 after transfection with miR-124 or miR-506 decreased IRF4 and k light chain proteins in H929 cells (Figure 7A), as determined by Western blot. Additionally, CD38 levels quantified by flow cytometry were also reduced (Figure 7B). These results prompted us to analyze the effect of miR-124 and miR-506 overexpression on myeloma cell morphology. We observed that the IRE1 knockdown induced by miR-124 and miR-506 overexpression led to changes in cell appearance, shaped like smaller, more rounded cells with a lower cytoplasm and ER content than control cells (Figure 7C). Together, these results confirm that the presence of IRE1 is required to maintain myeloma cells at the terminal stage of differentiation.

A

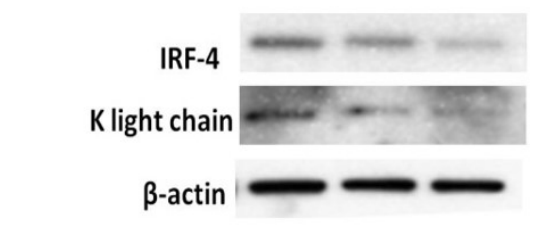

B

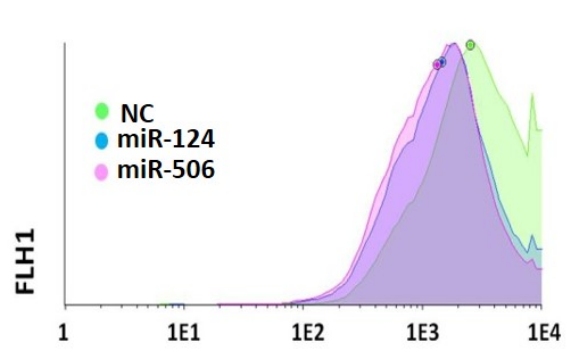

C

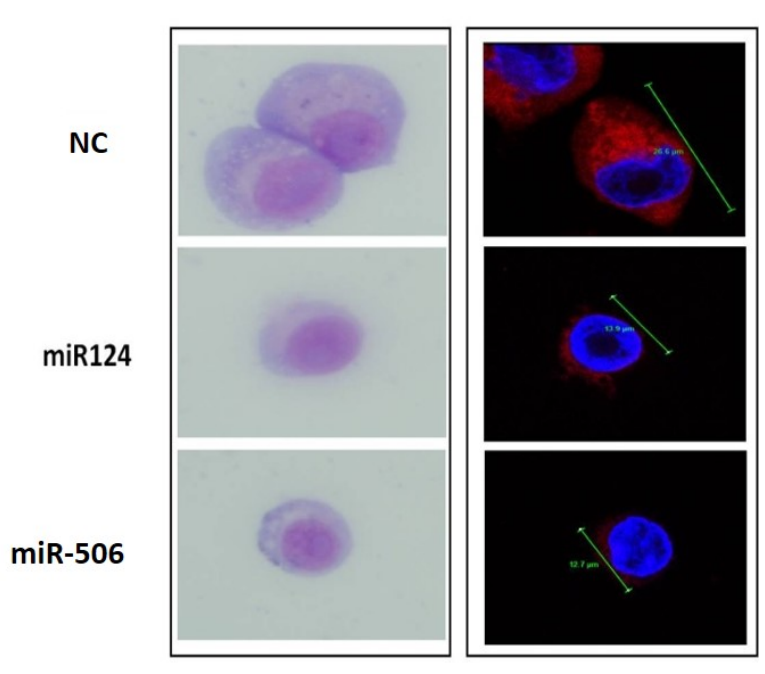

Figure 7. IRE1 knockdown induces dedifferentiation of myeloma cells. A Western blot of IRF4, kappa and lambda light chain in H929 72 h post-transfection with NC or miR-124/miR-506. B Flow cytometry analysis of CD38 in H929 after miR-124/506 overexpression compared with non-targeting control (NC). C Left panel, Giemsa staining of H929 cells transfected with NC or with miR-124/506. Right panel, immunofluorescence of H929 cells stained with ER tracker ${ }^{\text {TM }}$ Red dye. 


\section{Discussion}

In this study, we show that IRE1, an unfolded protein response (UPR) sensor with kinase and endoribonuclease activities ${ }^{23}$, is necessary to maintain PC status since its knockdown was associated with dedifferentiation of myeloma cells. Moreover, we found that miR-124 and miR506 modulate IRE1 expression in MM cells.

We achieved IRE1 knockdown by means of shRNA technique after several unsuccessful attempts using siRNA. Indeed, shRNA is significantly more potent than siRNA at mediating gene silencing 25,26 .

In the present work, we observed that IRE1 levels increased during the differentiation of human PCs from BCs. Accordingly, we found that IRE1 knockdown changed the transcriptional program associated with PC differentiation through the upregulation of PAX5 and BCL6, which maintain the $\mathrm{BC}$ program ${ }^{18}$. Therefore, we propose that IRE1 is not only required for antibody production in PCs, but also to maintain the state of PCs, and its deficiency results in PC dedifferentiation. Our results are in agreement with Leung-Hagesteijn et al who confirmed that the presence of the IRE1-XBP1 pathway was essential to maintain PC differentiation. Indeed, several studies have also revealed that alterations in other factors that control PC maturation may reprogram PCs to previous stages of differentiation $24,27-29$.

The involvement of miRNAs in the pathogenesis of MM has been demonstrated by several groups ${ }^{30-32}$. In this study, we demonstrate for the first time the regulation of IRE1 in MM by microRNAs. We found that IRE1 expression was controlled by miR-124 and miR-506. Using luciferase reporter assays and gain-of-function experiments, we showed that transfection of miR-124 and miR-506 decreased IRE1 levels in myeloma cells. Moreover, we observed that IRE1 downregulation induced by miR-124 and miR-506 ectopic expression also reverted the transcriptional program of the myeloma cells and reduced cell size and ER mass, similarly to the results obtained from IRE1 silencing by shRNA. These findings emphasize the role that these miRNAs play in regulating IRE1 expression and therefore, in PC differentiation program.

MiR-124 and miR-506 belong to the same seed family (defined as a group of miRNAs that share common seed sequence) ${ }^{33}$. The downregulation of these two miRNAs has been reported in several cancers, which has been associated with tumor progression. Specifically, the downregulation of miR-124 was reported in different tumors such as, esophageal cancer, breast cancer and renal cell carcinoma ${ }^{34-36}$. Furthermore, miR-506 expression was also shown to be downregulated in oral squamous cell carcinoma, nasopharyngeal carcinoma and gastric cancer ${ }^{37-39}$. In addition, the overexpression of miR-124 and miR-506 in colorectal cancer 
inhibits progression and increases sensitivity to chemotherapy by targeting DNMT3B and DNMT1 ${ }^{40}$.

After the publication by Leung-Hagesteijn et al ${ }^{24}$ demonstrating the role of IRE1-XBP1 signaling pathway in maintaining PC differentiation and in the mechanisms of proteasome inhibitor resistance in $\mathrm{MM}$, we decided to focus on other functions of IRE1, as an endoribonuclease enzyme, and worked in the identification of new mRNA targets of IRE1 in MM (chapter 3). 


\section{References}

1. Hetz C and Papa FR. The Unfolded Protein Response and Cell Fate Control. Mol Cell. 2018;69(2):169-181.

2. Walter $P$ and Ron D. The unfolded protein response: from stress pathway to homeostatic regulation. Science. 2011;334(6059):1081-1086.

3. Bettigole SE and Glimcher LH. Endoplasmic reticulum stress in immunity. Annu Rev Immunol. 2015;33:107-138.

4. Janssens S, Pulendran B, Lambrecht BN. Emerging functions of the unfolded protein response in immunity. Nat Immunol. 2014;15(10):910-919.

5. Yoshida H, Matsui T, Yamamoto A, Okada T, Mori K. XBP1 mRNA is induced by ATF6 and spliced by IRE1 in response to ER stress to produce a highly active transcription factor. Cell. 2001;107(7):881-891.

6. Calfon M, Zeng H, Urano F, et al. IRE1 couples endoplasmic reticulum load to secretory capacity by processing the XBP-1 mRNA. Nature. 2002;415(6867):92-96.

7. Lee AH, Iwakoshi NN, Glimcher LH. XBP-1 regulates a subset of endoplasmic reticulum resident chaperone genes in the unfolded protein response. Mol Cell Biol. 2003;23(21):7448-7459.

8. Lee AH, Chu GC, Iwakoshi NN, Glimcher LH. XBP-1 is required for biogenesis of cellular secretory machinery of exocrine glands. EMBO J. 2005;24(24):4368-4380.

9. Lipson KL, Fonseca SG, Ishigaki S, et al. Regulation of insulin biosynthesis in pancreatic beta cells by an endoplasmic reticulum-resident protein kinase IRE1. Cell Metab. 2006;4(3):245-254.

10. Iwawaki T, Akai R, Kohno K. IRE1alpha disruption causes histological abnormality of exocrine tissues, increase of blood glucose level, and decrease of serum immunoglobulin level. PLoS One. 2010;5(9):e13052.

11. Gass JN, Gunn KE, Sriburi R, Brewer JW. Stressed-out B cells? Plasma-cell differentiation and the unfolded protein response. Trends Immunol. 2004;25(1):17-24.

12. Zhang K, Wong HN, Song B, Miller CN, Scheuner D, Kaufman RJ. The unfolded protein response sensor IRE1alpha is required at 2 distinct steps in B cell lymphopoiesis. J Clin Invest. 2005;115(2):268-281.

13. Bartoszewska S, Kochan $\mathrm{K}$, Madanecki $\mathrm{P}$, et al. Regulation of the unfolded protein response by microRNAs. Cell Mol Biol Lett. 2013;18(4):555-578.

14. Su SF, Chang YW, Andreu-Vieyra C, et al. miR-30d, miR-181a and miR-199a-5p cooperatively suppress the endoplasmic reticulum chaperone and signaling regulator GRP78 in cancer. Oncogene. 2013;32(39):4694-4701.

15. Maurel M, Dejeans N, Taouji S, Chevet E, Grosset CF. MicroRNA-1291-mediated silencing of IRE1alpha enhances Glypican-3 expression. RNA. 2013;19(6):778-788. 
16. Gutierrez NC, Ocio EM, de Las Rivas J, et al. Gene expression profiling of B lymphocytes and plasma cells from Waldenstrom's macroglobulinemia: comparison with expression patterns of the same cell counterparts from chronic lymphocytic leukemia, multiple myeloma and normal individuals. Leukemia. 2007;21(3):541-549.

17. Shaffer AL, Shapiro-Shelef M, Iwakoshi NN, et al. XBP1, downstream of Blimp-1, expands the secretory apparatus and other organelles, and increases protein synthesis in plasma cell differentiation. Immunity. 2004;21(1):81-93.

18. Nutt SL, Hodgkin PD, Tarlinton DM, Corcoran LM. The generation of antibody-secreting plasma cells. Nat Rev Immunol. 2015;15(3):160-171.

19. Michallet AS, Mondiere P, Taillardet M, Leverrier $Y$, Genestier L, Defrance T. Compromising the unfolded protein response induces autophagy-mediated cell death in multiple myeloma cells. PLoS One. 2011;6(10):e25820.

20. Papandreou I, Denko NC, Olson M, et al. Identification of an Ire1alpha endonuclease specific inhibitor with cytotoxic activity against human multiple myeloma. Blood. 2011;117(4):1311-1314.

21. Mimura N, Fulciniti M, Gorgun G, et al. Blockade of XBP1 splicing by inhibition of IRE1alpha is a promising therapeutic option in multiple myeloma. Blood. 2012;119(24):5772-5781.

22. Misiewicz-Krzeminska I, Sarasquete ME, Quwaider D, et al. Restoration of microRNA214 expression reduces growth of myeloma cells through positive regulation of P53 and inhibition of DNA replication. Haematologica. 2013;98(4):640-648.

23. Lee KP, Dey M, Neculai D, Cao C, Dever TE, Sicheri F. Structure of the dual enzyme Ire1 reveals the basis for catalysis and regulation in nonconventional RNA splicing. Cell. 2008;132(1):89-100.

24. Leung-Hagesteijn C, Erdmann N, Cheung G, et al. Xbp1s-negative tumor B cells and preplasmablasts mediate therapeutic proteasome inhibitor resistance in multiple myeloma. Cancer Cell. 2013;24(3):289-304.

25. Rao DD, Vorhies JS, Senzer N, Nemunaitis J. siRNA vs. shRNA: similarities and differences. Adv Drug Deliv Rev. 2009;61(9):746-759.

26. McAnuff MA, Rettig GR, Rice KG. Potency of siRNA versus shRNA mediated knockdown in vivo. J Pharm Sci. 2007;96(11):2922-2930.

27. van Keimpema M, Gruneberg LJ, Mokry M, et al. The forkhead transcription factor FOXP1 represses human plasma cell differentiation. Blood. 2015;126(18):2098-2109.

28. Fujita N, Jaye DL, Geigerman C, et al. MTA3 and the Mi-2/NuRD complex regulate cell fate during B lymphocyte differentiation. Cell. 2004;119(1):75-86.

29. Mikkola I, Heavey B, Horcher M, Busslinger M. Reversion of B cell commitment upon loss of Pax5 expression. Science. 2002;297(5578):110-113. 
30. Gutierrez NC, Sarasquete ME, Misiewicz-Krzeminska I, et al. Deregulation of microRNA expression in the different genetic subtypes of multiple myeloma and correlation with gene expression profiling. Leukemia. 2010;24(3):629-637.

31. Pichiorri F, Suh SS, Ladetto M, et al. MicroRNAs regulate critical genes associated with multiple myeloma pathogenesis. Proc Natl Acad Sci U S A. 2008;105(35):12885-12890.

32. Lionetti M, Biasiolo M, Agnelli L, et al. Identification of microRNA expression patterns and definition of a microRNA/mRNA regulatory network in distinct molecular groups of multiple myeloma. Blood. 2009;114(25):e20-6.

33. Zhao Z, Ma X, Hsiao TH, et al. A high-content morphological screen identifies novel microRNAs that regulate neuroblastoma cell differentiation. Oncotarget. 2014;5(9):24992512.

34. Cheng Y, Li Y, Nian Y, Liu D, Dai F, Zhang J. STAT3 is involved in miR-124-mediated suppressive effects on esophageal cancer cells. BMC Cancer. 2015;15:306-015-1303-0.

35. Dong LL, Chen LM, Wang WM, Zhang LM. Decreased expression of microRNA-124 is an independent unfavorable prognostic factor for patients with breast cancer. Diagn Pathol. 2015;10:45-015-0257-5.

36. Long QZ, Du YF, Liu XG, Li X, He DL. miR-124 represses FZD5 to attenuate P-glycoproteinmediated chemo-resistance in renal cell carcinoma. Tumour Biol. 2015;36(9):7017-7026.

37. Deng L and Liu H. MicroRNA-506 suppresses growth and metastasis of oral squamous cell carcinoma via targeting GATA6. Int J Clin Exp Med. 2015;8(2):1862-1870.

38. Zhang Z, Ma J, Luan G, et al. MiR-506 suppresses tumor proliferation and invasion by targeting FOXQ1 in nasopharyngeal carcinoma. PLoS One. 2015;10(4):e0122851.

39. Deng J, Lei W, Xiang X, et al. MicroRNA-506 inhibits gastric cancer proliferation and invasion by directly targeting Yap1. Tumour Biol. 2015;36(9):6823-6831.

40. Chen Z, Liu S, Tian L, et al. miR-124 and miR-506 inhibit colorectal cancer progression by targeting DNMT3B and DNMT1. Oncotarget. 2015;6(35):38139-38150. 


\section{Supplementary materials}

Supplementary Tables:

Table S1. List of oligonucleotide sequences used for $3^{\prime}$ UTR luciferase constructs.

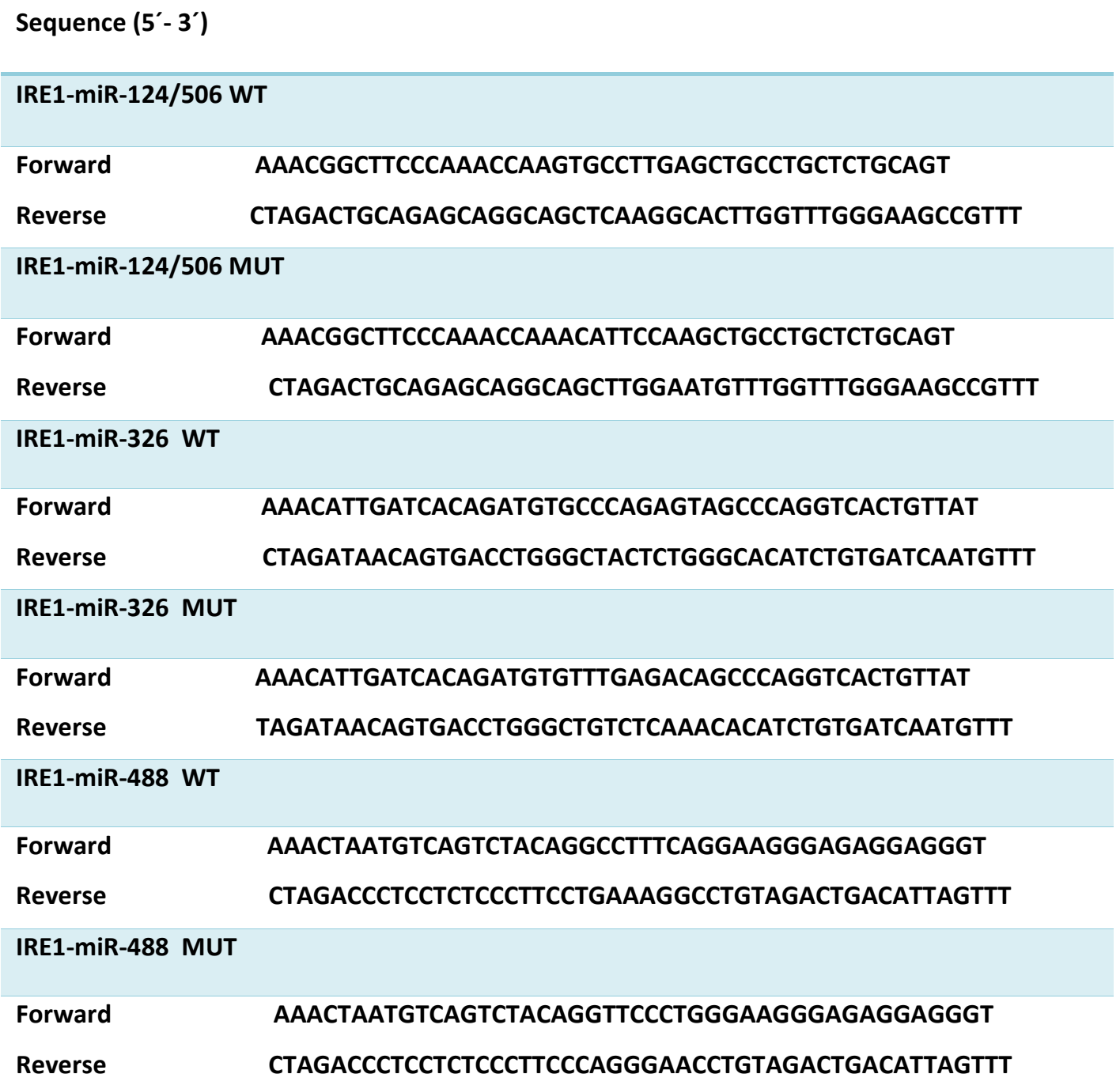





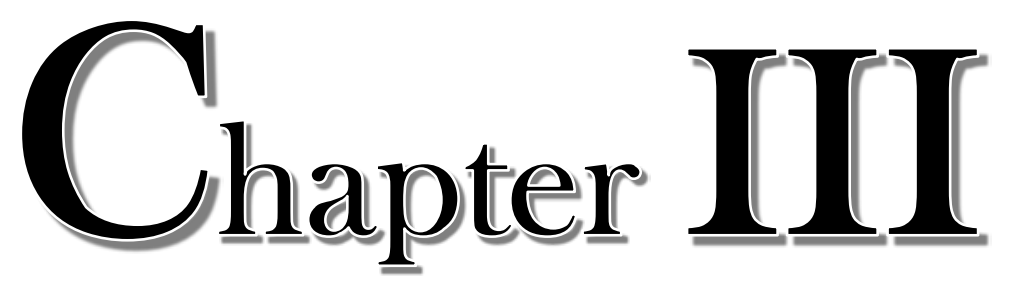

\section{RNA sequencing identifies novel regulated IRE1- dependent decay targets that affect multiple myeloma survival and proliferation}

Dalia Quwaider ${ }^{1,2.3}$, Luis A. Corchete ${ }^{1.2,3}$, Marta Matín-IzquierdoRamón ${ }^{1,2.3}$, Jesus M. Hernández-Sánchez ${ }^{1,2,3}$, Elizabeta A. Rojas ${ }^{1,2,3}$, Ramón García-Sanz ${ }^{1-4}$, Ana B. Herrero $^{1,2 \%}$, and Norma C. Gutiérrez ${ }^{1 .+4 t}$ 



\begin{abstract}
IRE1 is an unfolded protein response sensor with kinase and endonuclease activity. It plays a central role in the endoplasmic reticulum (ER) stress response through unconventional splicing of XBP1 mRNA and regulated IRE1-dependent decay (RIDD). MM cells are known to exhibit an elevated level of baseline ER stress, but RIDD activity has not been well studied in this disease. To investigate novel RIDD targets of possible relevance to the survival/proliferation of MM cells we combined in vitro cleavage assay with RNA sequencing. Bioinformatic analysis revealed hundreds of putative IRE1 substrates, 32 of which were chosen for validation. Looking into the secondary structure of IRE1 substrates, we found that the consensus sequences of IRF4, PRDM1, IKZF1, KLF13, NOTCH1, ATR, DICER, RICTOR, CDK12, FAM168B, and CENPF mRNAs were accompanied by a stem-loop structure essential for IRE1-mediated cleavage. We show that mRNA and protein levels corresponding to these targets were attenuated in an IRE1-dependent manner. Our results demonstrate for the first time that IRE1 is a key regulator of several proteins of importance in MM survival and proliferation.
\end{abstract}





\section{Introduction}

The endoplasmic reticulum (ER) plays an essential role in the folding and processing of newly synthesized proteins ${ }^{1}$. Certain environmental stimuli and pathological conditions can produce a quantity of unfolded proteins that exceeds the folding capacity of the ER. Under these circumstances, cells activate the ER stress response or unfolded protein response (UPR), an intracellular signaling pathway that adjusts protein-folding capacity to maintain ER homeostasis 2. IRE1, a bifunctional enzyme with kinase and endonuclease activity ${ }^{3}$, is a master regulator of the UPR pathway. ER stress triggers the oligomerization and trans-autophosphorylation of IRE1, switching on its RNase activity 4,5 . The primary target of IRE1 is the mRNA that encodes the X-box binding protein (XBP1). In mammalian cells, the full-length mRNA of XBP1, also referred to as the unspliced form (XBP1u), is cleaved by IRE1 at two specific sites, causing the removal of an unconventional intron of 26 nucleotides. This splicing event causes a frameshift that allows the translation of the spliced form (XBP1s), which encodes a potent transcription factor ${ }^{6}$. XBP1 induces the expression of various chaperones and other ER-resident proteins, which increases the folding capacity of the ER, and increases the level of membrane phospholipids, triggering its expansion ${ }^{7}$. In addition, activation of IRE1 has also been shown to target several mRNAs for degradation in an XBP1-independent manner. This mechanism, known as regulated IRE1-dependent decay (RIDD), was first demonstrated in Drosophila melanogaster ${ }^{8}$ and later in yeast species and mammalian cells ${ }^{9-11}$. RIDD is believed to have the potential to selectively relieve the load on the ER by mediating the cleavage and degradation of ER-targeted mRNAs ${ }^{8}$. IRE1 activity has been shown to regulate cell fate decisions, depending on the amplitude and duration of its activation, which, in turn, depends on the intensity of ER stress ${ }^{12}$. RIDD promotes apoptosis by degrading the mRNA-encoding proteins essential for cell survival ${ }^{12-}$ ${ }^{14}$ and select microRNAs that normally repress translation of Caspase $2{ }^{14}$. It is important to note that the RIDD of miRNAs during ER stress can also modulate the expression of hundreds of mRNA targets ${ }^{15}$.

The mechanism by which IRE1 recognizes and cleaves its targets became clear a decade ago, when Oikawa et al demonstrated that the cleavage site of 13 novel mRNAs RIDD targets contained a consensus sequence (CUGCAG) within a stem-loop structure that was also present in XBP1 mRNA ${ }^{16}$. Furthermore, it was shown that the consensus cleavage site and stem-loop structure are conserved in mammalian cells, as demonstrated in other known RIDD targets ${ }^{16-18 .}$

Several studies have suggested that many RIDD targets are yet to be identified, and that these may depend on the nature of the stress stimuli and the tissue context ${ }^{19}$. In this regard, 
multiple myeloma (MM) cells are a useful model for studying new targets of IRE1 since the secretion of high levels of paraprotein raises the basal level of ER stress ${ }^{20}$ and creates a dependency on the UPR for survival 21.

In this study, we combined an in vitro cleavage assay with RNA sequencing (RNA-seq) to identify the IRE1-cleavage site of new RIDD targets in MM. We identified hundreds of putative IRE1-mRNA substrates and validated 11 novel stem-loop-containing mRNA targets that were cleaved in vitro and in vivo by IRE1. mRNA and protein levels corresponding to these targets were attenuated under treatment with ER-stress-inducing agents in an IRE1dependent manner. 


\section{Materials and methods}

\section{Cell lines}

The NCI-H929 and MM1S human multiple myeloma cell lines (MMCLs) were acquired from ATCC (American Type Culture Collection). Cell line identity was confirmed periodically by STR analysis with the PowerPlex 16 HS system kit (www.promega.com) and online STR matching analysis (www.dsmz.de/fp/cgi-bin/str.html). Cell lines were cultured in RPMI 1640 medium supplemented with 10\% fetal bovine serum and antibiotics (Gibco Life Technologies, Grand Island, NY, USA).

\section{RNA extraction and in vitro RNA cleavage assay}

RNA was extracted from the cell lines using an RNeasy mini kit (Qiagen, Valencia, CA, USA) according to the standard protocol. RNA integrity was assessed using Agilent 2100 Bioanalyzer (Agilent Technologies, Santa Clara, CA, USA) and only RNA preparations with an RNA integrity number (RIN) > 7 were used. For the cleavage assay, total RNA ( $5 \mu \mathrm{g}$ ) was incubated for $1 \mathrm{~h}$ at $37^{\circ} \mathrm{C}$ in cleavage buffer (20 mM HEPES, pH 7.5, $50 \mathrm{mM} \mathrm{NaCl}, 1 \mathrm{mM}$ DTT, and $2 \mathrm{mM} \mathrm{ATP}$ ) in the presence or absence of $2 \mu \mathrm{g}$ of recombinant human ERN1/IRE1a (aa 467-977) (Sino Biological Inc., Beijing, China). The reaction was inactivated at $95^{\circ} \mathrm{C}$ for 5 min. RNA cleanup of these samples was performed using an RNeasy MinElute Cleanup Kit (Qiagen, Valencia, CA, USA), in accordance with the manufacturer's instructions.

\section{RNA sequencing}

The library for RNA-seq analysis was prepared using approximately $1.0 \mu \mathrm{g}$ of total RNA from each sample and the TruSeq Stranded mRNA Sample Preparation Kit version 1.0 (Illumina, San Diego, CA, USA), following the manufacturer's instructions. Briefly, mRNAs were purified using poly-T oligo-attached magnetic beads and the RNA was fragmented. The mRNA fragments were used as templates for first-strand cDNA synthesis by reverse transcription with random hexamers. Upon second-strand cDNA synthesis, doublestranded cDNAs were end-repaired and adenylated at the $3^{\prime}$ ends. Universal adapters were ligated to the cDNA fragments, then the sequencing library of DNA fragments that had adapters on both ends was amplified by PCR, and used to produce the clusters that were then sequenced in an Illumina HiSeq 2000 instrument (Illumina, San Diego, CA, USA). Each sample was sequenced in a separate flow cell lane, producing 26.2-32.4 million paired-end reads, with a final length of 76 bases. 


\section{RNA sequencing analysis}

The initial quality control of raw FASTQ files was performed using the FastQC tool (v.0.11.3) (http://www.bioinformatics.babraham.ac.uk/projects/fastqc). Adapter sequences and low-quality bases (Phred score $<20$ ) were trimmed from the raw reads using the Cutadapt (v1.12) program ${ }^{22}$. The processed reads were mapped to the reference human genome GRCh37 (Ensembl annotation, release 87) with the STAR aligner (v.2.5.3a), using default parameters. The resulting BAM alignment files were indexed and sorted using samtools (v.1.3.1) ${ }^{23}$.

Raw exon counts were obtained from the BAM files using the dexseq_count Python script available in the DEXSeq package (v. 1.24.4) ${ }^{24}$. Differential exon usage was analyzed analysis using a likelihood ratio (LR) test, and the corresponding graphs produced using the DEXSeq package. Unambiguous exons with a Benjamini \& Hochberg false discovery rate (FDR) < 0.05 threshold in the LR test and a negative fold change $(\mathrm{FC})<-2$ were considered for further analysis. Exons associated with two or more HGNC (HUGO Gene Nomenclature Committee) IDs were considered ambiguous. Pathway overrepresentation was measured with the WebGestalt web tool, using information about the HGNC gene correspondence with the selected exons and the Reactome pathway database ${ }^{25}$. Ensembl mRNA sequences from the transcripts containing the altered exons were used to predict the secondary mRNA structures on the Mfold web server ${ }^{26}$. The predicted secondary structure with the lowest thermodynamic energy was considered the most stable and, therefore, the most biologically likely structure. The dataset is available at the Gene Expression Omnibus (GEO) repository (http://www.ncbi.nlm.nih.gov/ geo) under the accession number GSE152070.

\section{Quantitative reverse transcriptase PCR (qRT-PCR)}

Total RNA (500 or $1000 \mu \mathrm{g}$ ) was reverse-transcribed to complementary DNA (cDNA) using SuperScript ${ }^{\mathrm{TM}}$ First-Strand Synthesis System for RT-PCR (Invitrogen, Waltham, MA, USA) with oligo (dt), or using a High-Capacity cDNA Reverse Transcription Kit (Applied Biosystems, Foster City, CA, USA).

Expression of target genes was quantified by qRT-PCR using an $\mathrm{iQ}^{\mathrm{TM}}$ SYBR ${ }^{\circledR}$ Green Supermix kit (Bio-Rad Laboratories, Hercules, CA, USA) and the primers shown in Supplementary Table S1. PCR melting curves were analyzed to confirm the presence of a single product. Relative gene expression was calculated by the $2^{-\Delta C t}$ method using GAPDH as the endogenous control for normalization. Each measurement was performed in triplicate. 


\section{RT-PCR analysis of XBP1 mRNA splicing}

RNA was reverse-transcribed to cDNA using a cDNA Reverse Transcription Kit from Applied Biosystems (Foster City, CA, USA). The PCR reaction was performed using GoTaq (Promega, Madison, WI, USA), following the manufacturer's protocol. The primers used were: XBP1 forward primer, 5'-TTACGAGAGAAAACTCATGGCC-3', and XBP1 reverse primer, 5'GGGTCCAAGTTGTCCAGAATGC-3'. The cycling conditions were $95^{\circ} \mathrm{C}$ for $5 \mathrm{~min}$, followed by 35 cycles of $95^{\circ} \mathrm{C}$ for $1 \mathrm{~min}, 58^{\circ} \mathrm{C}$ for $30 \mathrm{~s}$, and $72^{\circ} \mathrm{C}$ for $30 \mathrm{~s}$, then $72^{\circ} \mathrm{C}$ for $5 \mathrm{~min}$. Electrophoresis was performed using $5 \mu \mathrm{l}$ per sample in a $2 \%$ agarose gel.

\section{ER stress induction}

To induce ER stress, cells were seeded at a density of $1 \times 10^{6}$ cells/ml (NCI-H929 and MM1S) and incubated for 4 or $16 \mathrm{~h}$ with the following inducers: thapsigargin $(1.5 \mu \mathrm{M})$, tunicamycin $(10 \mu \mathrm{g} / \mathrm{ml})$, brefeldin A (600 ng/ml) (Sigma-Aldrich, St Louis, MO, USA), and dithiothreitol $(2 \mu \mathrm{M})$ (Promega, Madison, WI, USA). To inhibit IRE1 RNase activity, cells were treated with $15 \mu \mathrm{M} 4 \mu 8 \mathrm{c}$ (MedChemExpress, Monmouth Junction, NJ, USA).

\section{Western blot}

Whole cell lysates were collected using RIPA buffer (Sigma-Aldrich, St Louis, MO, USA) containing protease inhibitors (Complete Protease Inhibitor Cocktail Tablets; Santa Cruz Biotechnology, Delaware, CA, USA) and phosphatase inhibitors (Phosphatase Inhibitor Cocktail A and B; Santa Cruz Biotechnology, Delaware, CA, USA). Protein concentration was measured using the Bradford assay (BioRad Laboratories, Hercules, CA, USA). Protein samples $(30 \mu \mathrm{g} /$ lane) were subjected to sodium dodecyl sulfate-polyacrylamide gel electrophoresis (SDS-PAGE) and transferred to 0.45-nm polyvinylidene fluoride (PVDF) membranes (BioRad Laboratories, Hercules, CA, USA). The primary antibodies used for immunoblotting were: anti-IRF4 and anti-DICER from Santa Cruz Biotechnology (Delaware, CA, USA); anti-KLF13 from Novus Biologicals (Centennial, CO, USA); anti-ATR and antiCENPF from GeneTex (Irvine, CA, USA); and anti-Blimp1, anti-Ikaros, anti-NOTCH1 and antiGAPDH (used as an internal control for protein loading) from Cell Signaling Technology (Beverly, MA, USA). The membranes were then washed and incubated with the corresponding secondary horseradish peroxidase-linked antibodies: anti-mouse IgG, antirabbit IgG antibodies (Abcam, Cambridge, UK) or anti-goat IgG (Santa Cruz Biotechnology, Delaware, CA, USA) at 1:10,000. Chemiluminescence was detected using the Amersham ECL Plus ${ }^{\mathrm{TM}}$ WesternBlotting Detection Reagent (GE Healthcare, Chicago, IL, USA). 


\section{Results}

\section{Identification of RIDD targets in MM by RNA sequencing}

To identify mRNAs cleaved by IRE1 in MM we followed the steps shown in (Figure 1). An in vitro cleavage assay of the total RNA obtained from the H929 cell line was carried out in the presence or absence of recombinant IRE1 protein. To analyze the efficiency of the cleavage reaction, a part of mRNAs present in three IRE1-treated samples and three mock-treated controls were reverse-transcribed using an oligo(dT) primer, and mRNA levels of two known IRE1 targets, XBP1 and BLOC1S1 18, were quantified by qRT-PCR (Supplementary materials: Figure S1). Using a pair of primers that annealed on both sides of the IRE1cleaving sites in the tested molecules, markedly lower XBP1 and BLOC1S1 mRNA levels were observed in the three samples treated with IRE1, as expected. Then, we carried out RNA-sequencing from purified poly (A)-containing mRNAs. In this manner, reads mapping entire mRNA molecules were obtained from control samples. Conversely, IRE1-mRNA substrates lost the $5^{\prime}$ mRNA fragments during the purification process, which reduced the number of reads in these regions (Figure 1) giving rise to a scarcity of several exons. A bioinformatic analysis identified 1,859 unambiguous exons (Supplementary materials: Table S2), corresponding to 863 HGNC-annotated genes. These exhibited significantly lower read counts in IRE1-treated samples than in controls (FDR $<0.05, \mathrm{FC}<-2$ ). XBP1 was ranked $14^{\text {th }}$ among the underexpressed exons, based on FC (Table S2, K column). Reactome pathway enrichment analysis revealed that the putative mRNA targets were involved in a variety of pathways, such as membrane trafficking, cell cycle, DNA repair, and ubiquitination and proteasome degradation (Supplementary materials: Figure S2). 
Total RNA

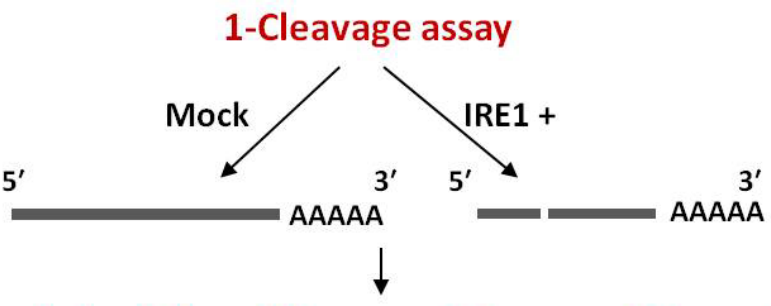

2- Analysis of the efficiency of cleavage (Figure S1).

3- Purification of poly(A) mRNAs with poly(T) magnetic beads and synthesis of cDNA

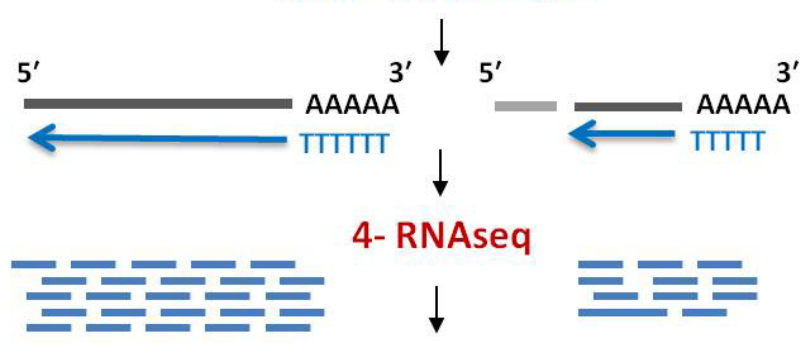

5- Bioinformatic analysis

1,859 exons $(\mathrm{FDR}<0.05, \mathrm{FC}<-2)$

6- Exon usage plot

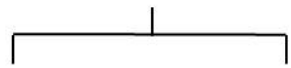

Identification of Identification of

IRE1 targets IRE1 cleavage site

Figure 1. Schematic representation of the cleavage reaction and RNA-sequencing procedure.

Total RNA from H929 cells was subjected to in vitro cleavage by the cytoplasmic domain of human IRE1 or to a mock treatment. The RNA was reverse-transcribed with oligo-dT primers and then RNAseq was performed. The lost $5^{\prime}$ side of the IRE1-mRNA substrates, represented in gray, is not reversetranscribed, producing fewer reads in the mRNA cleaved by IRE1 than in mock treatment samples. 
We plotted the exon usage values of the 863 genes, which allowed us to visualize all the exons in the $5^{\prime}$ region of the mRNAs with fewer reads in IRE1-treated samples than in controls. The exon-usage plot corresponding to the XBP1 mRNA showed that the number of reads corresponding to exons located $5^{\prime}$ to the cleavage sites was clearly lower in IRE1treated compared with untreated controls, whereas the number of reads was similar for the exons located in the $3^{\prime}$ region of both samples (Figure $2 \mathrm{~A}$ ). These findings were then validated by qRT-PCR using the cDNAs synthetized with oligo (dT) and a pair of primers that map the 3' region of the XBP1 mRNA molecule (Figure 2B, black arrows), or on both sides of the IRE1-cleavage sites on 5'region (Figure 2C, red arrows).

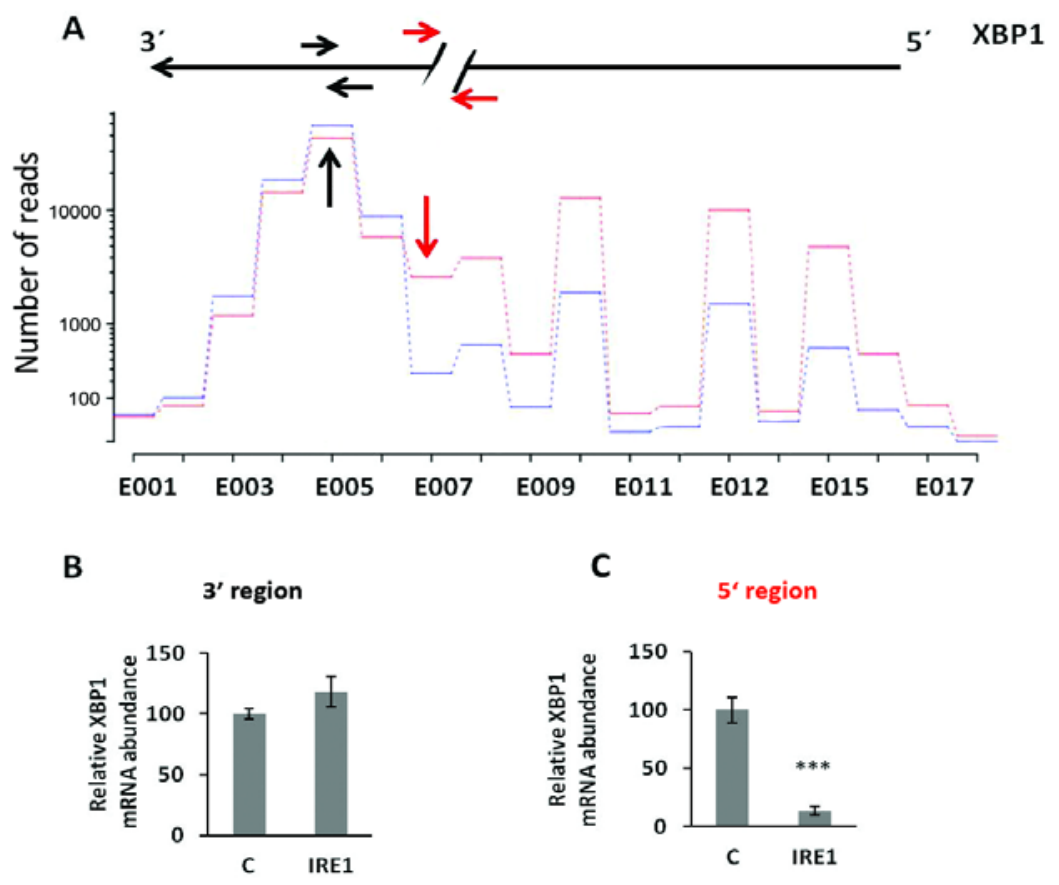

Figure 2. Differential exon usage of $X B P 1$ according to RNA-seq data. A Exon-usage plots of $X B P 1$ mRNA, which represents the number of reads in mock (red) and IRE1-treated (blue) samples. B XBP1 mRNA measured by qRT-PCR using the cDNAs synthesized with oligo (dT) and primers mapping the $5^{\prime}$ region (red arrow). C XBP1 mRNA measured by qRT-PCR using the cDNAs synthesized with oligo $(\mathrm{dT})$ and primers mapping the $3^{\prime}$ region (black arrow). All results are presented as the means $\pm \mathrm{SD}$ of three experiments. $\left.{ }^{* * *} p<0.001\right)$. 
Table 1. Selected putative IRE1 substrates.

\begin{tabular}{|c|c|c|}
\hline $\begin{array}{l}\text { Gene } \\
\text { symbol }\end{array}$ & FC & Function \\
\hline ATM & -34.9 & Regulation of DNA damage response \\
\hline VPS13C & -15.96 & Regulation of mitocondrial function \\
\hline BIRC6 & -12.04 & Ubiquitination and proteosomal protein involved in ubiquitin activity \\
\hline КМт2C & -9.94 & Protein methylation \\
\hline HUWE1 & -7.91 & Ubiquitination and proteosomal protein involved in ubiquitin activity \\
\hline RICTOR & -6.79 & TOR signaling \\
\hline CDK12 & -6.55 & Cyclin dependent kinase 12 \\
\hline CENPF & -6.03 & Cell cycle regulation and apoptosis induction \\
\hline VPS13D & -6.01 & Membrane trafficking \\
\hline GOLGB1 & -6.00 & Golgi to plasma membrane transport \\
\hline FAM168B & -5.81 & Inhibitor \\
\hline ATR & -5.60 & Regualtion of DNA damage response \\
\hline AKAP9 & -5.34 & Cell cycle regulation and apoptosis induction \\
\hline NOTCH1 & -4.86 & $\begin{array}{l}\text { Transcription factors (Cell fate determination, proliferation, } \\
\text { differentiation, apoptosis) }\end{array}$ \\
\hline PCM1 & -3.84 & Cell cycle regulation and apoptosis induction \\
\hline UBR2 & -3.60 & Ubiquitination and proteosomal protein involved in ubiquitin activity \\
\hline KLF13 & -3.44 & $\begin{array}{l}\text { Transcription factors (Cell fate determination, proliferation, } \\
\text { differentiation, apoptosis) }\end{array}$ \\
\hline mTOR & -3.03 & TOR signaling \\
\hline DICER & -2.99 & Ribonuclease III activity \\
\hline XRN1 & -2.90 & Regualtion of DNA damage response \\
\hline CUL9 & -2.81 & Ubiquitination and proteosomal protein involved in ubiquitin activity \\
\hline UBE4B & -2.68 & Ubiquitination and proteosomal protein involved in ubiquitin activity \\
\hline PSME4 & -2.47 & Ubiquitination and proteosomal protein involved in ubiquitin activity \\
\hline CUL4B & -2.36 & Ubiquitination and proteosomal protein involved in ubiquitin activity \\
\hline UBR3 & -2.35 & Ubiquitination and proteosomal protein involved in ubiquitin activity \\
\hline UBA6 & -2.33 & Ubiquitination and proteosomal protein involved in ubiquitin activity \\
\hline IKZF1 & -2.28 & $\begin{array}{l}\text { Transcription factors (Cell fate determination, proliferation, } \\
\text { differentiation, apoptosis) }\end{array}$ \\
\hline PSMD1 & -2.24 & Ubiquitination and proteosomal protein involved in ubiquitin activity \\
\hline CUL5 & -2.05 & Ubiquitination and proteosomal protein involved in ubiquitin activity \\
\hline ERAP1 & -2.00 & $\begin{array}{l}\text { Protein processing and transport. Cleaves other proteins into smaller } \\
\text { fragments. }\end{array}$ \\
\hline PRDM1 & -1.71 & Transcription factor implicated in the differentiation of PC \\
\hline IRF4 & -1.59 & Transcription factor implicated in the differentiation of PC \\
\hline
\end{tabular}


Of the 863 genes with an $\mathrm{FC}<-2$, we selected for validation those exhibiting the greatest FC between the control samples and those treated with IRE1, and in turn those that showed a similar exon-usage plot to that of XBP1. A total of 30 genes were selected by these criteria (Table 1). We also included IRF4 and PRDM1 genes in the validation analysis, based on their known function in PC differentiation and in the pathogenesis of MM, even though both genes had a FC >-2 (Table 1 ). We designed primers complementary to the $5^{\prime}$ region of the putative IRE1 substrates (Figure 3A, marked with a black arrow) and measured the amount of mRNA after the in vitro cleavage reaction by qRT-PCR. We found that the $5^{\prime}$ regions of the mRNAs from all 32 IRE1-substrate candidates significantly decreased in IRE1-treated samples compared to controls (four representative examples are shown in Figure 3A; the others are illustrated in (Supplementary materials Figure S3). Notably, IRE1 did not cleave the mRNA of the housekeeping genes $G A P D H$ and $B 2 M$, demonstrating that this protein is not a random nonspecific RNase (Figure 3B).

Together, these results indicate that RNA-seq is a suitable method for identifying new targets of IRE1 in the cells. 
A
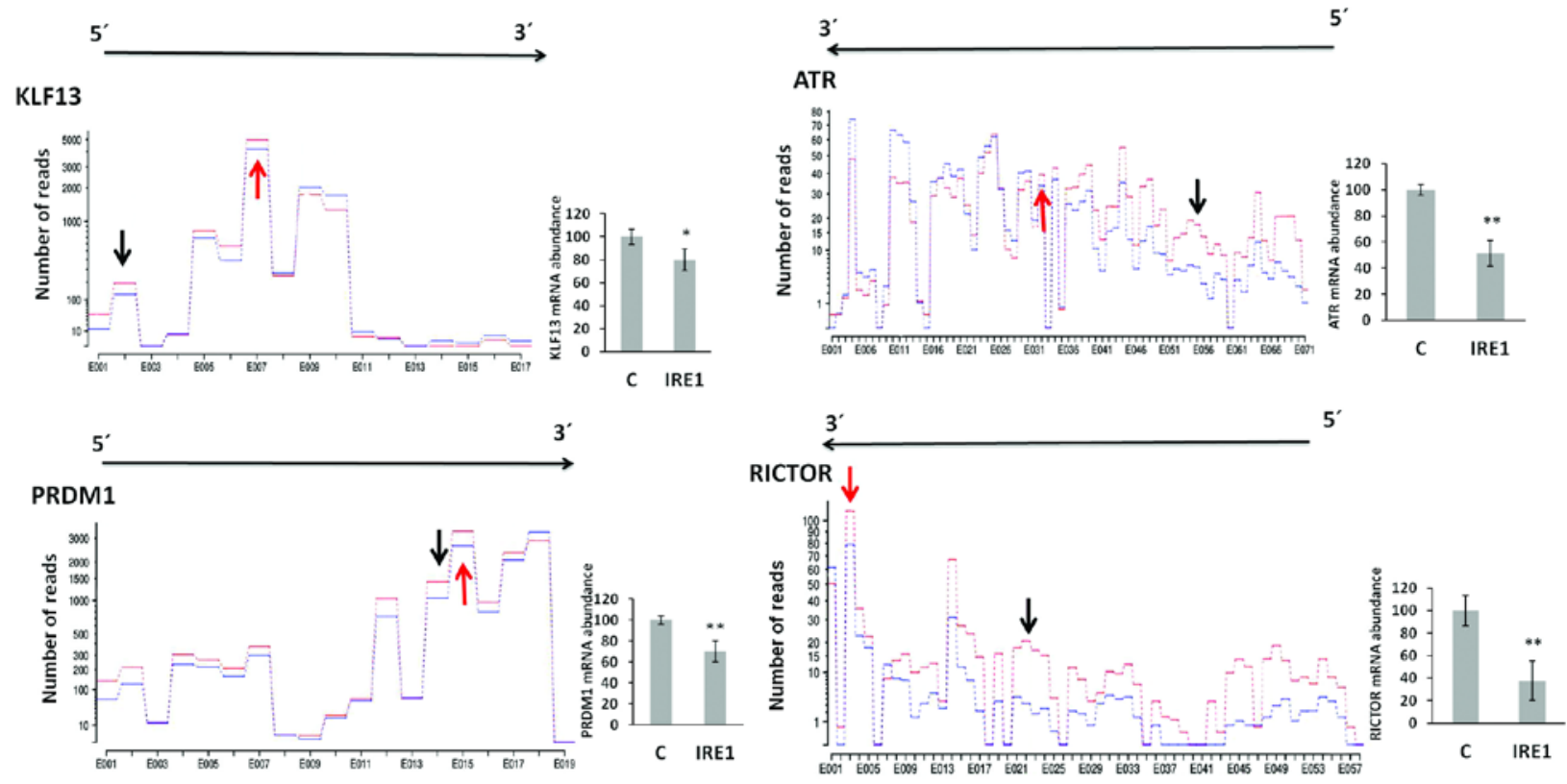

B GAPDH
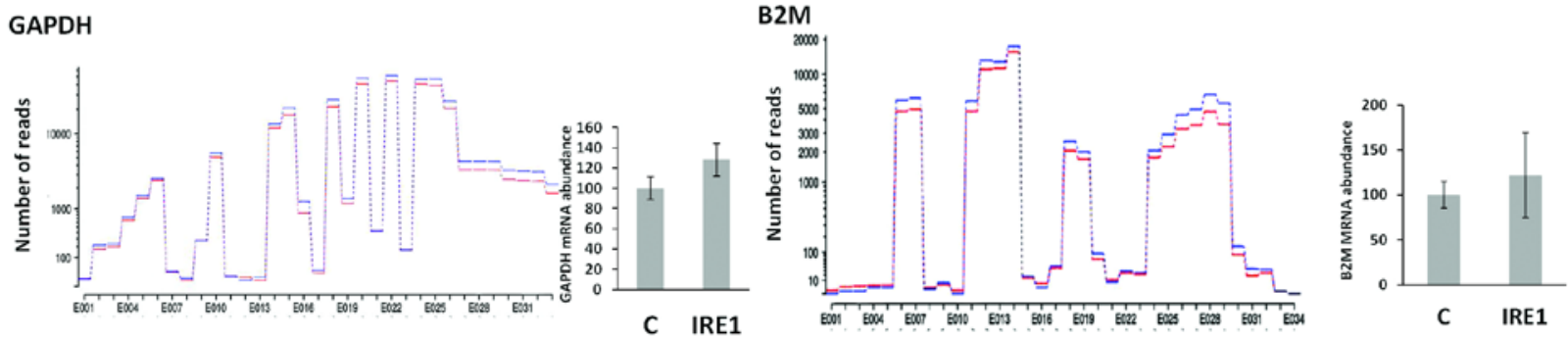

Figure 3. Validation of putative IRE1 substrates. (A) Exon-usage plots of $K L F 13, A T R, P R D M 1$, and RICTOR mRNAs, showing the number of reads in mock (red) and IRE1-treated (blue) samples. The black arrows represent the site of primers used in the $5^{\prime}$ region of the putative IRE1 substrates. Red arrows indicate the site of primers mapping the predicted cleavage site. Right panel of each exonusage plot shows the abundance of KLF13, ATR, PRDM1, and RICTOR mRNA measured by qRT-PCR using the cDNAs synthesized with oligo (dT). (B) GAPDH and B2M were used as negative controls, and their mRNA was measured by qRT-PCR. All results are presented as the means \pm SD of three experiments. $\left({ }^{*} p<0.05,{ }^{* *} p<0.01,{ }^{* * *} p<0.001\right)$. 


\section{Identification of the IRE1 cleavage site in RIDD targets}

It has been shown that the cleavage activity of IRE1 is a sequence-specific event occurring at the XBP1-like consensus site ${ }^{16}$. We examined whether the newly identified IRE1 targets contained the consensus CUGCAG sequence within an XBP1-like stem loop structure 16. First, the secondary structures of the 32 mRNAs identified as IRE1 substrate candidates were obtained using Mfold, and then a search for stem-loop structures near the cleavage regions was undertaken. The cleavage region for each IRE1-target mRNA was identified from the previously generated exon-usage plots, since it must correspond to the first region that exhibits fewer reads in IRE1-treated samples than in mock samples (Figure 3 and Supplementary Figure S3, red arrows). Some molecules displayed putative loops, but outside the identified IRE1-cleavage site. Following this procedure, we found that 11 of the 32 mRNAs (34\%) exhibited a clear stem-loop structure that contained the consensus sequence around the IRE1-cleavage site (Figure 4A and 4B). Some of the targets exhibited the IRE1-cleavage site in the coding regions, whereas others have the XBP1-like stem loop at the $3^{\prime}$ UTR region. On the other hand, most of the mRNAs contained one cleavage site; PRDM1 and ATR contained two cleavage sites located close to each other (Figure 4A and $4 \mathrm{~B})$.

To confirm that the 11 mRNAs were cleaved by IRE1 at the consensus sequence within the loop structure, we designed primers on both sides of this sequence and performed qRT-PCR (Figure 3A and Supplementary Figure S3, red arrows). We found that the loop region of these mRNAs was lost in these targets according to the PCR results, which revealed that the mRNA levels of all 11 IRE1 targets were significantly lower in IRE1-treated samples than in the controls (Figure 4C).

Overall, these results indicate that RNAseq is a suitable method not only for identifying new targets of IRE1, but also for mapping the IRE1-cleavage site. 
A

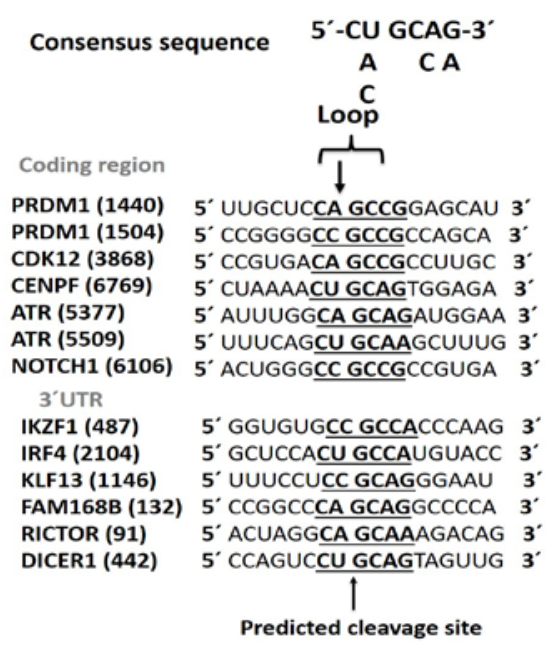

C
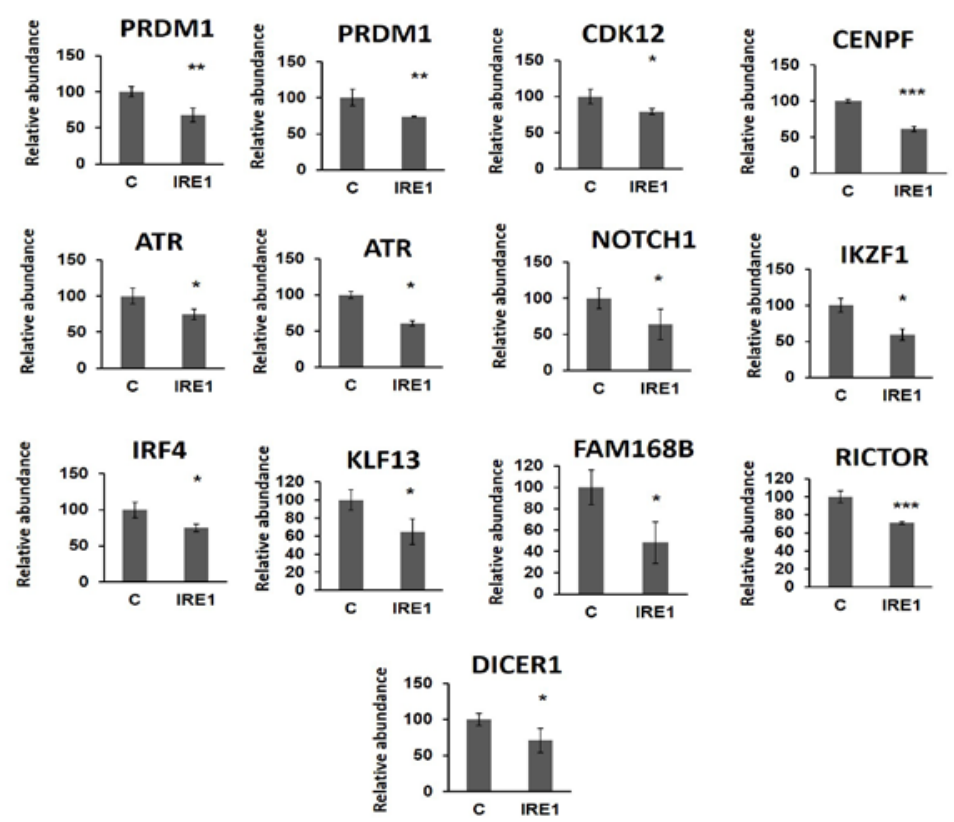
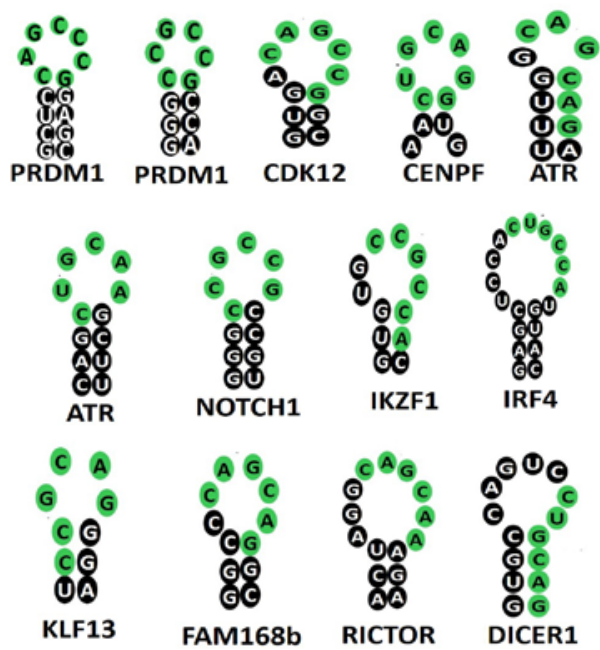

Figure 4. Mapping the cleavage site of IRE1 targets. A mRNA sequence around the consensus sequence of the newly identified IRE1 targets. The consensus sequence is underlined. The numbers indicate the guanine position at the consensus sequence, where IRE1 is supposed to cleave. B Schematic representation of the secondary mRNA structures containing the consensus sequence of the identified targets of IRE1. C mRNA levels of the indicated genes in H929 using cDNAs synthesized with oligo (dT) determined by qRT-PCR using primers mapping the predicted cleavage site. All results are presented as the means \pm SD of three experiments. $\left({ }^{*} p<0.05,{ }^{* *} p<0.01,{ }^{* * *} p<0.001\right)$. 


\section{IRE1 degrades the identified RIDD targets in MM in response to ER stress}

There is controversy over whether the targets of IRE1 cleavage are degraded upon ER stress induction. This has been observed for BLOC1S1 and CD59 mRNA targets 17, 18, but not for others ${ }^{16}$. We investigated this aspect in the 11 RIDD targets identified in this study by treating H929 cells with one of four ER stress inducers (thapsigargin, tunicamycin, dithiothreitol or brefeldin A) for $4 \mathrm{~h}{ }^{27}$. To confirm that the putative mRNA degradation was IRE1-dependent, the experiment was carried out in the presence or absence of the IRE1 RNase inhibitor $4 \mu 8 \mathrm{c}{ }^{28}$. Previously, the induction of ER stress in H929 cells had been verified by the analysis of the spliced/unspliced forms of XBP1 (Figure 5A), and by measuring the levels of BLOC1S1 mRNA (Figure 5B). All four agents clearly induced the splicing of XBP1 and reduced BLOC1S1 mRNA levels in an IRE1-dependent manner, since both effects were reverted in the presence of $4 \mu 8 \mathrm{c}$.

We quantified the 11 targets by qRT-PCR using the primers surrounding the previously identified cleavage sites. We found that the mRNA levels of KLF13, DICER, NOTCH1, IRF4, and ATR were reduced upon treatment with some of the ER inducers, then recovered in the presence of $4 \mu 8 \mathrm{c}$ (Figure 5C). No stress-dependent decay was detected with the other RIDD targets under the conditions assayed. We also analyzed the effect of ER-stress induced by thapsigargin at the protein level using western blot at different times. We found that KLF13, DICER, NOTCH1, IRF4, and ATR protein expression had not changed by $4 \mathrm{~h}$ post-treatment, whereas it had clearly decreased by $16 \mathrm{~h}$ (Figure 5D). This result prompted us to speculate that the effect of stress-dependent decay could be better detected after a longer time. Consequently, we analyzed protein levels of BLIMP1 (encoded by PRDM1), IKAROS (encoded by IZKF1) and CENPF, whose mRNAs did not display decay $4 \mathrm{~h}$ after treatment, after a long exposure $(16 \mathrm{~h})$ to thapsigargin. As shown in Figure 5D, the levels of these proteins were also lower in thapsigargin-treated cells than in the untreated controls. The FAM168B and CDK12 proteins could not be analyzed because the commercial antibodies were no sufficiently specific.

Finally, we decided to check the protein levels of the newly identified RIDD targets in another MM cell line with a different genetic background, like MM1S, after thapsigargin exposure for 4 and $16 \mathrm{~h}$. Similar to the results obtained in H929, protein levels of RIDD substrates had not changed after $4 \mathrm{~h}$, with the exception of KLF13 and CENPF, but had clearly decreased after $16 \mathrm{~h}$ of thapsigargin treatment (Figure 5D). Importantly, levels of GAPDH did not change during the course of the experiment, again revealing that GAPDH mRNA is not an IRE1 target, and that IRE1 is not a random non-specific RNase. 
Together, our results show that the mRNAs of KLF13, DICER, NOTCH1, IRF4, ATR, BLIMP1, IKAROS and CENPF genes are novel RIDD targets in MM and that the stress-induced degradation of these mRNAs is IRE1-dependent. 


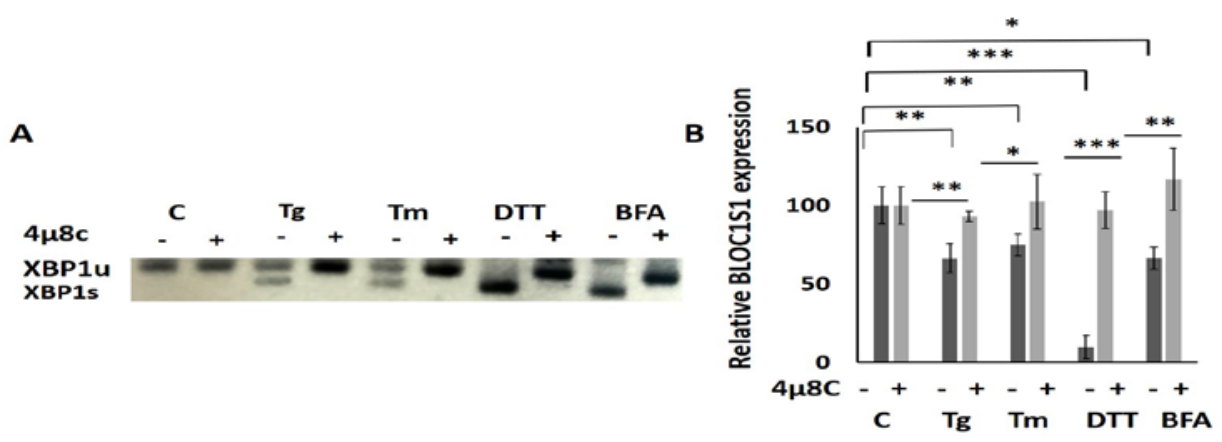

C

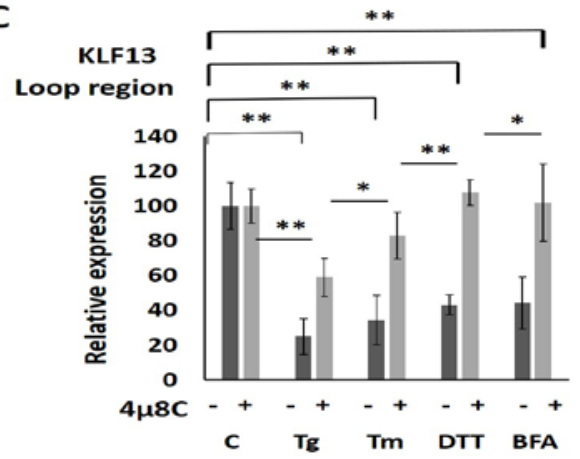

DICER
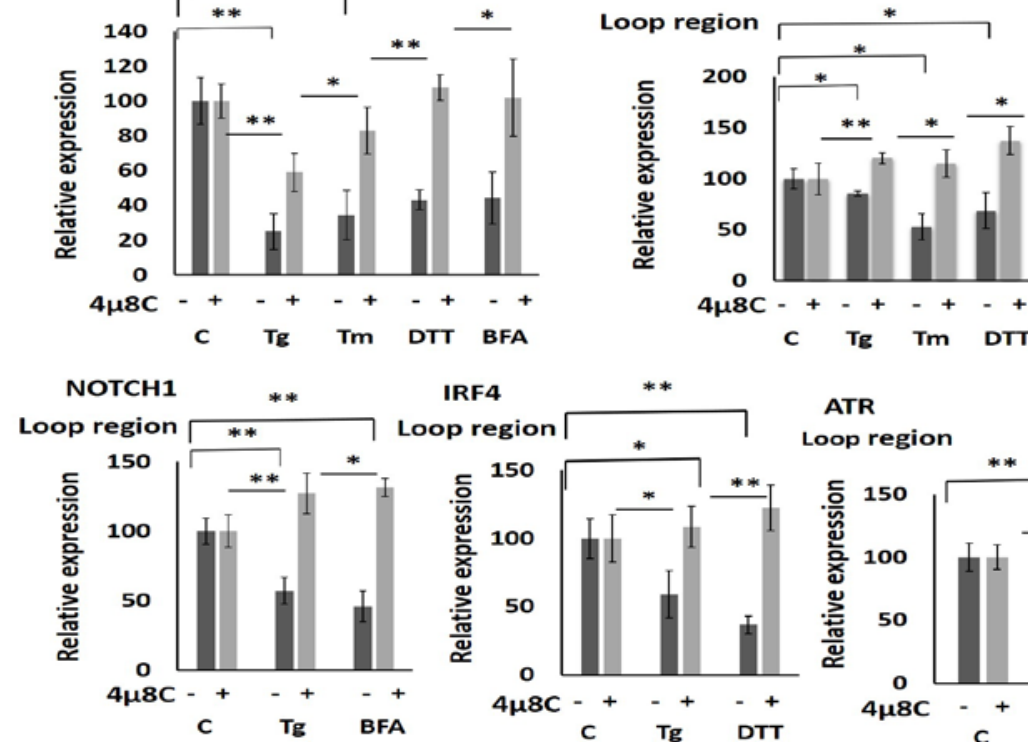

IRF4

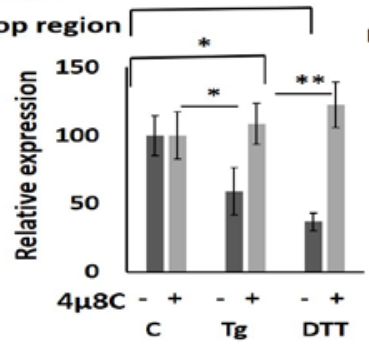

ATR

Loop region

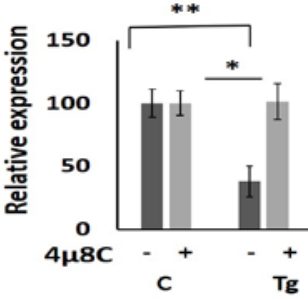

D

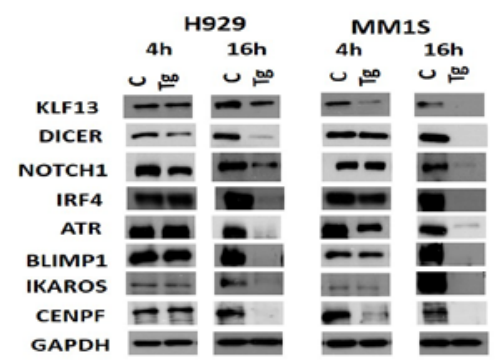

Figure 5. RIDD targets in MM. A Representative agarose gel electrophoresis of the PCR product surrounding the XBP1 splice site in H929. B BLOC1S1 mRNA measured by qRT-PCR. C mRNA levels of the indicated genes were determined by qRT-PCR. H929 cells were treated with different ER-

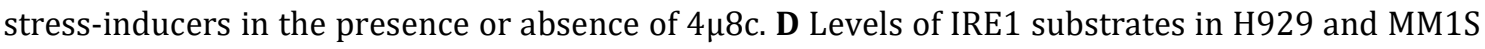
after $4 \mathrm{~h}$ and $16 \mathrm{~h}$ of thapsigargin treatment. All results are presented as the means \pm SD of three experiments. $\left({ }^{*} p<0.05,{ }^{* *} p<0.01,{ }^{* *} p<0.001\right)$. 


\section{Discussion}

IRE1 is a key component of cell fate switch because it produces either adaptive/pro-survival or death signals, depending on the intensity and duration of ER stress. This occurs through unconventional splicing of XBP1 mRNA, and by RIDD 29, 30. MM cells are characterized by a massive production of immunoglobulins that raise the baseline level of ER stress ${ }^{21}$. This probably explains the sensitivity of myeloma cells to proteasome inhibitors (PIs) like bortezomib, which exacerbate ER stress ${ }^{31}$. In this study, we have identified novel RIDD targets and their IRE1-cleavage sites in MM cell lines through a combination of in vitro cleavage assay and RNA-sequencing. We found that IRF4, PRDM1, IKZF1, KLF13, NOTCH1, ATR, DICER, RICTOR, CDK12, FAM168B, and CENPF mRNAs had the consensus sequence (CUGCAG) accompanied by a stem-loop structure essential for IRE1-mediated cleavage. In addition, we show that all these targets degraded in vivo after induction of ER stress by IRE1. The cleavage activity of IRE1 is a sequence-specific event occurring within the XBP1-like stem structure ${ }^{16}$. The consensus sequence and the stem loop structure are also conserved in previously identified IRE1 targets, such as CD59, Sparc, and PER1, which also showed mRNA decay in an IRE1-dependent manner 17, 32, 33. In myeloma cells, the only RIDD target that had already been identified was BLOC1S1 mRNA, which contains an XBP1-like stem loop, and is degraded upon ER stress induction by IRE1 at guanine $444{ }^{18}$. While we also confirmed this finding, the novel contribution of our study is to identify several key genes in MM as RIDD targets by taking advantage of the greater potential and sensitivity of RNAseq. In addition, the IRE1-dependent degradation of these targets was demonstrated in in vivo studies.

All the 11 targets identified in our study had at least one cleavage site. Notably, PRDM1 and $A T R$ had two cleavages sites on their mRNAs, as described for XBP1. However, unlike XBP1, we did not detect a new mRNA product resulting from the removal of the sequence between the cutting sites (data not shown), which suggests that IRE1 cuts both sites only to promote mRNA degradation.

RIDD activity becomes cytotoxic after prolonged and unmitigated exposure to ER stress ${ }^{30}$. Some of the RIDD targets identified in this study, such as NOTCH1, KLF13, ATR, CENPF, IRF4, and IKZF1, encode proteins involved in the survival/proliferation of MM cells, so their ER stress-mediated degradation may promote cell death. For example, NOTCH1 inhibition in MM induces apoptosis and reduces the proliferation rate 34,35 . NOTCH blockade also makes MM cells more sensitive to standard chemotherapies ${ }^{34}$ and to pro-apoptotic compounds such as Bcl-2/Bcl-XL inhibitors ${ }^{36}$. KLF13 belongs to the Krüppel-like factor (KLF) family, which is formed by zinc finger transcription factors that have been involved in proliferation, 
differentiation, cell cycle progression, and apoptosis ${ }^{37-39}$. The role of KLF13 in MM has not yet been established, but it is tempting to speculate that the UPR-mediated downregulation observed in our study might have diminished MM cell proliferation, giving time for the ER stress to have been alleviated. It had been previously shown that ER stress reduces DNA double-strand break (DSB) repair and increases radiosensitivity of tumor cells via proteasomal degradation of Rad51 ${ }^{40}$. Here, we found that $A T R$, which has a well established role in the signaling and repair of DSBs ${ }^{41}$ is a target of IRE1. We found that the mRNA encoding other proteins with essential roles in DNA damage signaling and repair, such as ATM, Rad50, and Xrn1 (Supplementary Table S1), were also downregulated upon treatment with the IRE1 recombinant enzyme. These results suggest that ER stress might prevent DSB repair by several means, which might act as part of a pro-apoptotic signaling mechanism that is triggered by severe DNA damage.

Some other RIDD targets validated in this study, such as PRDM1, IRF4, and IKZF1, are mRNAs involved in B cell maturation. BLIMP1 and IRF4 along with XBP1 are the three pillars that maintain plasma cell differentiation status ${ }^{42}$, and IKAROS is a transcription factor that regulates early-lymphoid cell development and promotes B-cell lineage maturation ${ }^{43}$. Expression of BLIMP1 or XBP1 is sufficient to drive BC differentiation towards Ig-secreting PCs, although their expression is not required for PC survival 44,45 . In contrast, the survival of PC critically depends on IRF4 ${ }^{46}$, and IKAROS knockdown inhibits proliferation and induces apoptosis of MM cell lines ${ }^{47}$. In fact, IKAROS is one of the proteins most strongly downregulated after treatment with immunomodulatory drugs (IMiDs) and it must be degraded for these agents to exert their cytotoxic effect ${ }^{48-50}$. Some studies have revealed that patients with a low level of IKZF1 expression had significantly better survival than those with a higher level of expression. It has also been suggested that MM cells with a low level of IKAROS expression are more sensitive to therapy ${ }^{51,52}$. The analysis of the mechanisms regulating IKAROS expression may be particularly important for understanding how resistance to IMiDs develops ${ }^{50}$. For our part, we found that IKAROS downregulation may be the result of the induction of ER stress.

The relevance of XBP1s to MM pathogenesis has been previously reported ${ }^{45}$. Two other studies have demonstrated that the blockade of IRE1-XBP1 axis by IRE1 alpha inhibitors induces anti-MM cytotoxicity and enhances sensitivity of MM cells to PI 53,54. Conversely, another study showed that silencing of either IRE1 or XBP1 not only failed to impair MM cell growth, but also promoted resistance to bortezomib ${ }^{45}$. The authors found that the weaker response to bortezomib was related to maturation arrest in the plasma cells, as indicated by the repression of plasma cell maturation markers, the smaller quantity of 
immunoglobulin produced, and the lower levels of UPR activation. In keeping with this, a low level of XBP1 gene expression in MM patients has been associated with poor response to bortezomib treatment 55 . Our results are consistent with this line of research in the sense that the RNase activity of IRE1 induced degradation of proteins involved in MM cell proliferation. Therefore, the role of IRE1 in maintaining plasma cell differentiation and secretory immunoglobulin production in addition to the downregulation of proteins and transcription factors that sustain MM cell survival, suggests that IRE1 inhibitors may not be a proper approach for treating MM. Further research to test the potential of IRE1 activity as a predictive biomarker of sensitivity to anti-myeloma therapy is needed. 


\section{References}

1. Braakman I and Hebert DN. Protein folding in the endoplasmic reticulum. Cold Spring Harb Perspect Biol. 2013;5(5):a013201.

2. Ron D and Walter P. Signal integration in the endoplasmic reticulum unfolded protein response. Nat Rev Mol Cell Biol. 2007;8(7):519-529.

3. Prischi F, Nowak PR, Carrara M, Ali MM. Phosphoregulation of Ire1 RNase splicing activity. Nat Commun. 2014;5:3554.

4. Oikawa D, Kimata Y, Kohno K, Iwawaki T. Activation of mammalian IRE1alpha upon ER stress depends on dissociation of BiP rather than on direct interaction with unfolded proteins. Exp Cell Res. 2009;315(15):2496-2504.

5. Promlek T, Ishiwata-Kimata Y, Shido M, Sakuramoto M, Kohno K, Kimata Y. Membrane aberrancy and unfolded proteins activate the endoplasmic reticulum stress sensor Ire1 in different ways. Mol Biol Cell. 2011;22(18):3520-3532.

6. Yoshida H, Matsui T, Yamamoto A, Okada T, Mori K. XBP1 mRNA is induced by ATF6 and spliced by IRE1 in response to ER stress to produce a highly active transcription factor. Cell. 2001;107(7):881-891.

7. He Y, Sun S, Sha H, et al. Emerging roles for XBP1, a sUPeR transcription factor. Gene Expr. 2010;15(1):13-25.

8. Hollien J and Weissman JS. Decay of endoplasmic reticulum-localized mRNAs during the unfolded protein response. Science. 2006;313(5783):104-107.

9. Miyazaki T, Nakayama H, Nagayoshi Y, Kakeya H, Kohno S. Dissection of Ire1 functions reveals stress response mechanisms uniquely evolved in Candida glabrata. PLoS Pathog. 2013;9(1):e1003160.

10. Kimmig P, Diaz M, Zheng J, et al. The unfolded protein response in fission yeast modulates stability of select mRNAs to maintain protein homeostasis. Elife. 2012;1:e00048.

11. Hollien J, Lin JH, Li H, Stevens N, Walter P, Weissman JS. Regulated Ire1-dependent decay of messenger RNAs in mammalian cells. J Cell Biol. 2009;186(3):323-331. 
12. Han D, Lerner AG, Vande Walle L, et al. IRE1alpha kinase activation modes control alternate endoribonuclease outputs to determine divergent cell fates. Cell. 2009;138(3):562-575.

13. Hetz C. The unfolded protein response: controlling cell fate decisions under ER stress and beyond. Nat Rev Mol Cell Biol. 2012;13(2):89-102.

14. Upton JP, Wang L, Han D, et al. IRE1alpha cleaves select microRNAs during ER stress to derepress translation of proapoptotic Caspase-2. Science. 2012;338(6108):818-822.

15. Maurel $\mathrm{M}$ and Chevet E. Endoplasmic reticulum stress signaling: the microRNA connection. Am J Physiol Cell Physiol. 2013;304(12):C1117-26.

16. Oikawa D, Tokuda M, Hosoda A, Iwawaki T. Identification of a consensus element recognized and cleaved by IRE1 alpha. Nucleic Acids Res. 2010;38(18):6265-6273.

17. Oikawa D, Tokuda M, Iwawaki T. Site-specific cleavage of CD59 mRNA by endoplasmic reticulum-localized ribonuclease, IRE1. Biochem Biophys Res Commun. 2007;360(1):122127.

18. Bright MD, Itzhak DN, Wardell CP, Morgan GJ, Davies FE. Cleavage of BLOC1S1 mRNA by IRE1 Is Sequence Specific, Temporally Separate from XBP1 Splicing, and Dispensable for Cell Viability under Acute Endoplasmic Reticulum Stress. Mol Cell Biol. 2015;35(12):21862202.

19. Dejeans N, Manie S, Hetz C, et al. Addicted to secrete - novel concepts and targets in cancer therapy. Trends Mol Med. 2014;20(5):242-250.

20. Carrasco DR, Sukhdeo K, Protopopova M, et al. The differentiation and stress response factor XBP-1 drives multiple myeloma pathogenesis. Cancer Cell. 2007;11(4):349-360.

21. Aronson LI and Davies FE. DangER: protein ovERload. Targeting protein degradation to treat myeloma. Haematologica. 2012;97(8):1119-1130.

22. Martin M. Cutadapt removes adapter sequences from high-throughput sequencing reads. :DOI: https://doi.org/10.14806/ej.17.1.200.

23. Dobin A, Davis CA, Schlesinger F, et al. STAR: ultrafast universal RNA-seq aligner. Bioinformatics. 2013;29(1):15-21. 
24. Anders S, Reyes A, Huber W. Detecting differential usage of exons from RNA-seq data. Genome Res. 2012;22(10):2008-2017.

25. Liao Y, Wang J, Jaehnig EJ, Shi Z, Zhang B. WebGestalt 2019: gene set analysis toolkit with revamped UIs and APIs. Nucleic Acids Res. 2019;47(W1):W199-W205.

26. Zuker M. Mfold web server for nucleic acid folding and hybridization prediction. Nucleic Acids Res. 2003;31(13):3406-3415.

27. Oslowski CM and Urano F. Measuring ER stress and the unfolded protein response using mammalian tissue culture system. Methods Enzymol. 2011;490:71-92.

28. Cross BC, Bond PJ, Sadowski PG, et al. The molecular basis for selective inhibition of unconventional mRNA splicing by an IRE1-binding small molecule. Proc Natl Acad Sci U S A. 2012;109(15):E869-78.

29. Chen Y and Brandizzi F. IRE1: ER stress sensor and cell fate executor. Trends Cell Biol. 2013;23(11):547-555.

30. Maurel M, Chevet E, Tavernier J, Gerlo S. Getting RIDD of RNA: IRE1 in cell fate regulation. Trends Biochem Sci. 2014;39(5):245-254.

31. Dong H, Chen L, Chen X, et al. Dysregulation of unfolded protein response partially underlies proapoptotic activity of bortezomib in multiple myeloma cells. Leuk Lymphoma. 2009;50(6):974-984.

32. Dejeans N, Pluquet O, Lhomond S, et al. Autocrine control of glioma cells adhesion and migration through IRE1alpha-mediated cleavage of SPARC mRNA. J Cell Sci. 2012;125(Pt 18):4278-4287.

33. Pluquet O, Dejeans N, Bouchecareilh M, et al. Posttranscriptional regulation of PER1 underlies the oncogenic function of IREalpha. Cancer Res. 2013;73(15):4732-4743.

34. Nefedova Y, Sullivan DM, Bolick SC, Dalton WS, Gabrilovich DI. Inhibition of Notch signaling induces apoptosis of myeloma cells and enhances sensitivity to chemotherapy. Blood. 2008;111(4):2220-2229. 
35. Mirandola L, Apicella L, Colombo M, et al. Anti-Notch treatment prevents multiple myeloma cells localization to the bone marrow via the chemokine system CXCR4/SDF-1. Leukemia. 2013;27(7):1558-1566.

36. Li M, Chen F, Clifton N, et al. Combined inhibition of Notch signaling and Bcl-2/Bcl-xL results in synergistic antimyeloma effect. Mol Cancer Ther. 2010;9(12):3200-3209.

37. Chen X, Johns DC, Geiman DE, et al. Kruppel-like factor 4 (gut-enriched Kruppel-like factor) inhibits cell proliferation by blocking G1/S progression of the cell cycle. J Biol Chem. 2001;276(32):30423-30428.

38. Kaczynski J, Cook T, Urrutia R. Sp1- and Kruppel-like transcription factors. Genome Biol. 2003;4(2):206-2003-4-2-206. Epub 2003 Feb 3.

39. Nemer M and Horb ME. The KLF family of transcriptional regulators in cardiomyocyte proliferation and differentiation. Cell Cycle. 2007;6(2):117-121.

40. Yamamori T, Meike S, Nagane M, Yasui H, Inanami O. ER stress suppresses DNA doublestrand break repair and sensitizes tumor cells to ionizing radiation by stimulating proteasomal degradation of Rad51. FEBS Lett. 2013;587(20):3348-3353.

41. Marechal A and Zou L. DNA damage sensing by the ATM and ATR kinases. Cold Spring Harb Perspect Biol. 2013;5(9):10.1101/cshperspect.a012716.

42. Nutt SL, Taubenheim N, Hasbold J, Corcoran LM, Hodgkin PD. The genetic network controlling plasma cell differentiation. Semin Immunol. 2011;23(5):341-349.

43. Sellars M, Kastner P, Chan S. Ikaros in B cell development and function. World J Biol Chem. 2011;2(6):132-139.

44. Tellier J, Shi W, Minnich M, et al. Blimp-1 controls plasma cell function through the regulation of immunoglobulin secretion and the unfolded protein response. Nat Immunol. 2016;17(3):323-330.

45. Leung-Hagesteijn C, Erdmann N, Cheung G, et al. Xbp1s-negative tumor B cells and preplasmablasts mediate therapeutic proteasome inhibitor resistance in multiple myeloma. Cancer Cell. 2013;24(3):289-304. 
46. Shaffer AL, Emre NC, Lamy L, et al. IRF4 addiction in multiple myeloma. Nature. 2008;454(7201):226-231.

47. Bjorklund CC, Lu L, Kang J, et al. Rate of CRL4(CRBN) substrate Ikaros and Aiolos degradation underlies differential activity of lenalidomide and pomalidomide in multiple myeloma cells by regulation of c-Myc and IRF4. Blood Cancer J. 2015;5:e354.

48. Kronke J, Udeshi ND, Narla A, et al. Lenalidomide causes selective degradation of IKZF1 and IKZF3 in multiple myeloma cells. Science. 2014;343(6168):301-305.

49. Lu G, Middleton RE, Sun H, et al. The myeloma drug lenalidomide promotes the cereblondependent destruction of Ikaros proteins. Science. 2014;343(6168):305-309.

50. Zhu YX, Braggio E, Shi CX, et al. Identification of cereblon-binding proteins and relationship with response and survival after IMiDs in multiple myeloma. Blood. 2014;124(4):536-545.

51. Kronke J, Kuchenbauer F, Kull M, et al. IKZF1 expression is a prognostic marker in newly diagnosed standard-risk multiple myeloma treated with lenalidomide and intensive chemotherapy: a study of the German Myeloma Study Group (DSMM). Leukemia. 2017;31(6):1363-1367.

52. Kronke J, Knop S, Langer C. Prognostic impact of Ikaros expression in lenalidomidetreated multiple myeloma. Oncotarget. 2017;8(63):106163-106164.

53. Papandreou I, Denko NC, Olson M, et al. Identification of an Ire1alpha endonuclease specific inhibitor with cytotoxic activity against human multiple myeloma. Blood. 2011;117(4):1311-1314.

54. Mimura N, Fulciniti M, Gorgun G, et al. Blockade of XBP1 splicing by inhibition of IRE1alpha is a promising therapeutic option in multiple myeloma. Blood. 2012;119(24):5772-5781.

55. Ling SC, Lau EK, Al-Shabeeb A, et al. Response of myeloma to the proteasome inhibitor bortezomib is correlated with the unfolded protein response regulator XBP- 1 . Haematologica. 2012;97(1):64-72. 
Supplementary materials

Supplementary Tables:

Table S1. List of primer sequences used for RT-PCR analysis. F: Forward primer. R: Reverse primer.

\begin{tabular}{|c|c|c|}
\hline & Primer name & Primer sequance $\left(5^{\prime}-3^{\prime}\right)$ \\
\hline \multirow[t]{2}{*}{ VPS13C } & VPS13C F & TGGACAGACTCAAAGCTCTCT \\
\hline & VPS13C R & CCTGGTTGATATCATTGTTATAAA \\
\hline \multirow[t]{2}{*}{ ATM } & ATM F & GCAGAAACACTCCCAGCTT \\
\hline & ATM R & AGGTGTTTGGTGAGAATGTCC \\
\hline \multirow[t]{2}{*}{ BIRC6 } & BIRC6 F & TCAAAGAGGGAACCTTCCAA \\
\hline & BIRC6 R & GGACATGGAGACTGAAGAAACA \\
\hline \multirow[t]{3}{*}{ HUWE1 } & HUWE1 F & TGATGAAGGTGAAGAGGGAGA \\
\hline & HUWE1 R & CATGTTGTCAAACTCAATGATGA \\
\hline & CDK12 F & CAGTTCACGCAGTCGTCATT \\
\hline \multirow[t]{3}{*}{ CDK12 } & CDK12 R & GGGGCAGATTTTTCCAATTT \\
\hline & CDK12 LOOP F & TCTGGTTGAAGGCGATCTTT \\
\hline & CDK12 LOOP R & GTTTCAGGCCCATCAGTGTT \\
\hline \multirow[t]{2}{*}{ VPS13D } & VPS13D F & AGTGGGCATGGCAAATAGAG \\
\hline & VPS13D R & CAGAATTCCCAATGCTGACA \\
\hline \multirow[t]{4}{*}{ GOLGB1 } & GOLGB1 F & TGAGGAGAATATTGCTTCTTTGG \\
\hline & GOLGB1 R & CTCAGCTTTCAGCTCCTCCA \\
\hline & FAM168B F & CTGTATGCAGCACCTCCTCA \\
\hline & FAM168B R & GTGACCCCGTTGCCTCTA \\
\hline \multirow[t]{4}{*}{ FAM168B } & FAM168B LOOP F & ATTATTGGGGATTCCACAGC \\
\hline & FAM168B LOOP R & CAGGCAGTCCACAAAACAGA \\
\hline & NOTCH1 F & AGAATGACGCTCGTACCTGC \\
\hline & NOTCH1 R & ACAAGAGCCCGTTGAATTTG \\
\hline \multirow[t]{2}{*}{ NOTCH1 } & NOTCH1 LOOP F & GCTGGAGGACCTCATCAACT \\
\hline & NOTCH1 LOOP R & TCTCCTCCCTGTTGTTCTGC \\
\hline \multirow[t]{2}{*}{ UBR2 } & UBR2 F & GGATCCTCTTGTTCATTTATCAGAA \\
\hline & UBR2 R & TCTGCTGGCAATTCACTTTC \\
\hline \multirow[t]{3}{*}{ mTOR } & mTOR F & AGTCAAGAGGAGTCTACTCGC \\
\hline & mTOR R & GCCAAGATGCCACCTTTCCT \\
\hline & DICER F & TGGCAAACAAGATCCAGAGCT \\
\hline \multirow[t]{3}{*}{ DICER } & DICER R & AAACGAACCACCAAGTTGCA \\
\hline & DICER LOOP F & CCTCCAGCAGTCCCTAGGAT \\
\hline & DICER LOOP R & CCGGGAACATCACCTTACAC \\
\hline \multirow[t]{2}{*}{ CUL9 } & CUL9 F & CGGAGATCCCCACTTTTGT \\
\hline & CUL9 R & AGTATTCAGCATCAGGATCACG \\
\hline \multirow[t]{2}{*}{ UBE4B } & UBE4B F & GGACTTGATTGGCCAGATTT \\
\hline & UBE4B R & CTGGATTGGAGCCAGTGTTT \\
\hline
\end{tabular}


Table S1

\begin{tabular}{|c|c|c|}
\hline \multirow[t]{2}{*}{ PMSE4 } & PMSE4 F & CTCGAGTGGATGGAAGGAA \\
\hline & PMSE4 R & AGGCTTGTCAAAGCCACC \\
\hline \multirow[t]{2}{*}{ CUL4B } & CUL4B F & CTTCAACCTCGTCCTTCTGC \\
\hline & CUL4B R & GTTGCAGCAGTTGGTGAAGA \\
\hline \multirow[t]{2}{*}{ UBR3 } & UBR3 F & GGTTCGTCCCAAAACTTCAA \\
\hline & UBR3 R & GTCATCAAGCCAAGGAGGAT \\
\hline \multirow[t]{3}{*}{ UBA6 } & UBA6 $\mathrm{F}$ & ACAATGGGCACTAAGGGACA \\
\hline & UBA6 $\mathbf{R}$ & TCTCTTGCCCACTGTATGGT \\
\hline & IKZF1 F & AGAGCAACAACGAGGAGCAG \\
\hline \multirow[t]{3}{*}{ IKZF1 } & IKZF1 R & ACGTGATCCAGGAAGAGCAC \\
\hline & IKZF1 LOOP F & CAGTCCCCAGAAGCAGAGA \\
\hline & IKZF1 LOOP R & GACTTTCCCCAACCAATTCA \\
\hline \multirow[t]{2}{*}{ PSMD1 } & PSMD1 F & CAAACAATGTGTGGAAAATGC \\
\hline & PSMD1 R & CTTCGTGTCTCCAGAGCAA \\
\hline \multirow[t]{3}{*}{ CUL5 } & CUL5 F & GTTAACCTTTGTTCTAATCCTGA \\
\hline & CUL5 R & TGTACACCATTTTGTTGTAAATACG \\
\hline & IRF4 F & GCTTGGGCACTGTTTAAAGG \\
\hline \multirow[t]{3}{*}{ IRF4 } & IRF4 R & TTGTACGGGTCTGAGATGTCC \\
\hline & IRF4 LOOP F & TTGCACACACTTTCATGCAG \\
\hline & IRF4 LOOP R & TGCTGCCCAATACATATCC \\
\hline \multirow[t]{3}{*}{ ERAP1 } & ERAP1 F & GATGGGACACCATTTCCTTG \\
\hline & ERAP1 R & СTCTCTCCAGCTCCCTTCCT \\
\hline & KLF13 F & CCGCAGAGGAAGCACAA \\
\hline \multirow[t]{4}{*}{ KLF13 } & KLF13 R & AAGGGCCTCTCACCTGTG \\
\hline & KLF13 LOOP F & AGCAAGAGACCGGAACATTG \\
\hline & KLF13 LOOP R & AGTCTGAGTTGGGGACATGG \\
\hline & PRDM1 F & CCCAAAGAATGTCCCAAAGA \\
\hline \multirow[t]{4}{*}{ PRDM1 } & PRDM1 R & GGGCTCCCACGTCTTCTAA \\
\hline & PRDM1 LOOP F & TGCTCAACCCСАСТTCTCTC \\
\hline & PRDM1 LOOP R & GCTACAGGCCTTGTCCTTCA \\
\hline & RICTOR F & ACTCCAAATATGTTGACTTGATTG \\
\hline \multirow[t]{3}{*}{ RICTOR } & RICTOR R & CTTTGGTTACTCCGACGAACA \\
\hline & RICTOR LOOP F & AGAAGCAGAGGCTGTGTTGG \\
\hline & RICTOR LOOP R & CCAGTAACTGCGGAACAGT \\
\hline
\end{tabular}


Table S1

\begin{tabular}{|c|c|c|}
\hline & Primer name & Primer sequance $\left(5^{\prime}-3^{\prime}\right)$ \\
\hline \multirow{3}{*}{ ATR } & ATR F & TGCAGCTTCTGCTCTCATTC \\
\hline & ATR R & GTGTTCTCCAATACCGCAGCA \\
\hline & ATR LOOP F & ATGCTAACAGGTCCGAGTGG \\
\hline \multirow{3}{*}{ AKAPg } & ATR LOOP R & TGGTTTGATGCTATGCTCC \\
\hline & AKAP9 $\mathrm{F}$ & AGCCATCATTGCCTCTGAAA \\
\hline & AKAP9 R & TGTTCAGAATCAGGGCTCAG \\
\hline \multirow[t]{3}{*}{ PCM1 } & PCM1 F & ATGGAGAGCAGCCAGATTG \\
\hline & PCM1 R & GGAGGCTGAGAATCACGAAG \\
\hline & CENPF F & GATCAATTGAAGGAGCTCACA \\
\hline \multirow[t]{3}{*}{ CENPF } & CENPF R & GCCTGGTGTTTCTTTTCAGC \\
\hline & CENPF LOOP F & GAAAAACAAGGTCAGTTGTCAGAA \\
\hline & CENPF LOOP R & GGGCTCTCAGCTTTTCAATG \\
\hline \multirow[t]{2}{*}{ XRN1 } & XRN1 F & GAGATGAATATGGATTACCCTCTCA \\
\hline & XRN1 R & TCAGTTACTGGTGTGGATACAGG \\
\hline \multirow[t]{2}{*}{ КМT2C } & KMT2C F & ATCAACCCAATCGCTCATTC \\
\hline & KMT2C R & CGGCTTGTTGAGGAAGCTCAC \\
\hline \multirow[t]{2}{*}{ XBP1 3'region } & XBP1 F & GTTGGGCATTCTGGACAACT \\
\hline & XBP1 $R$ & CTTCCAGCTTGGCTGATGAC \\
\hline \multirow[t]{2}{*}{$B 2 M$} & B2M F & CTCACGTCATCCAGCAGAGA \\
\hline & B2M R & CGGCAGGCATACTCATCTTT \\
\hline \multirow[t]{2}{*}{ BLOC1S1 } & BLOC1S1 F & CCCAATTTGC CAAGCAGACA \\
\hline & BLOC1S1 R & CATCCCCAATTTCCTT GAGTGC \\
\hline \multirow[t]{2}{*}{ GAPDH } & GAPDH F & GGGTGGAATCATATTGGAACATGTA \\
\hline & GAPDH R & CAGGGCTGCTTTTAACTCTGGTAA \\
\hline
\end{tabular}

Table S2. List of differential usage of exons according to RNA-seq data-can be consulted through the following link:

(https://www.dropbox.com/sh/qdkoi4ky1tkzy5i/AAAXIseVTZh I3qWmUz9TXqVa?dl=0) 


\section{Supplementary Figures}

A
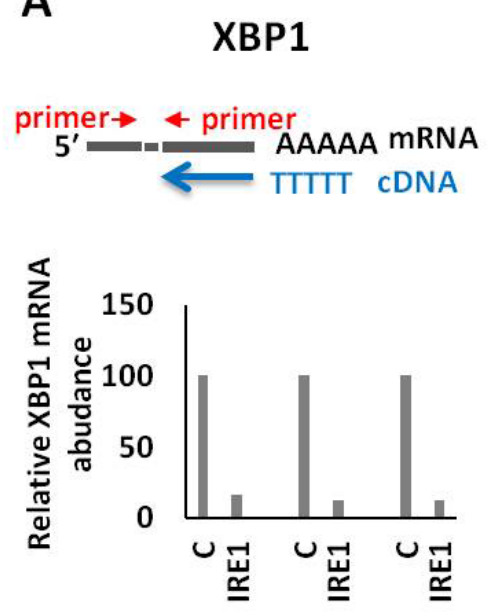

B
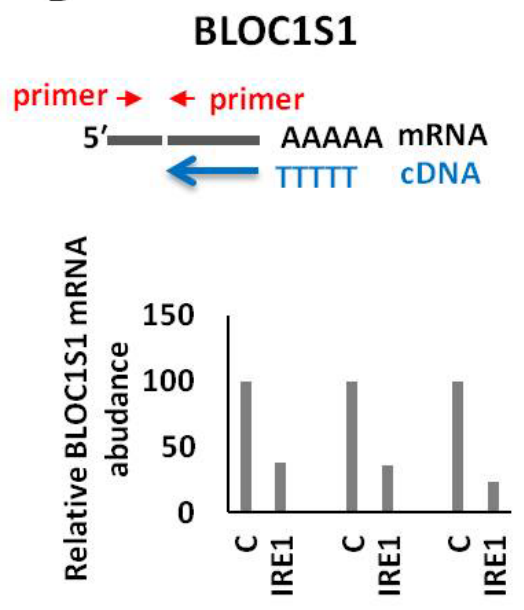

Figure S1. Efficiency of cleavage reaction. A XBP1 and B BLOC1S1 mRNA levels measured by qRTPCR using the cDNAs synthesized with oligo (dT), and primers mapping the cleavage site of IRE1 from mock and IRE1-treated samples. 


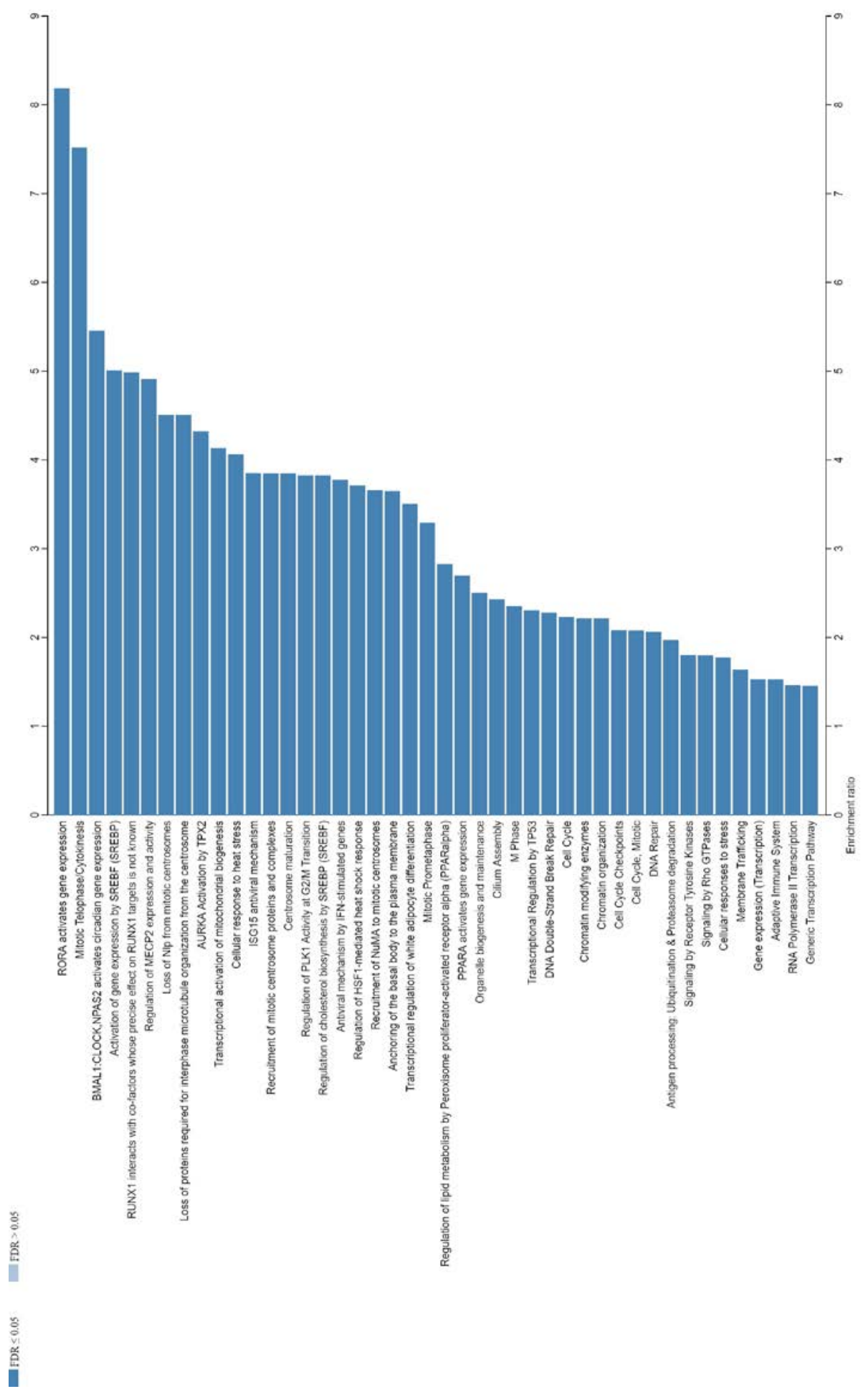

Figure S2. Reactome pathway analysis using RNA-seq data. Bar chart representing the most significantly enriched pathways. FDR $\leq 0.05$. 
Figure S3

ATM
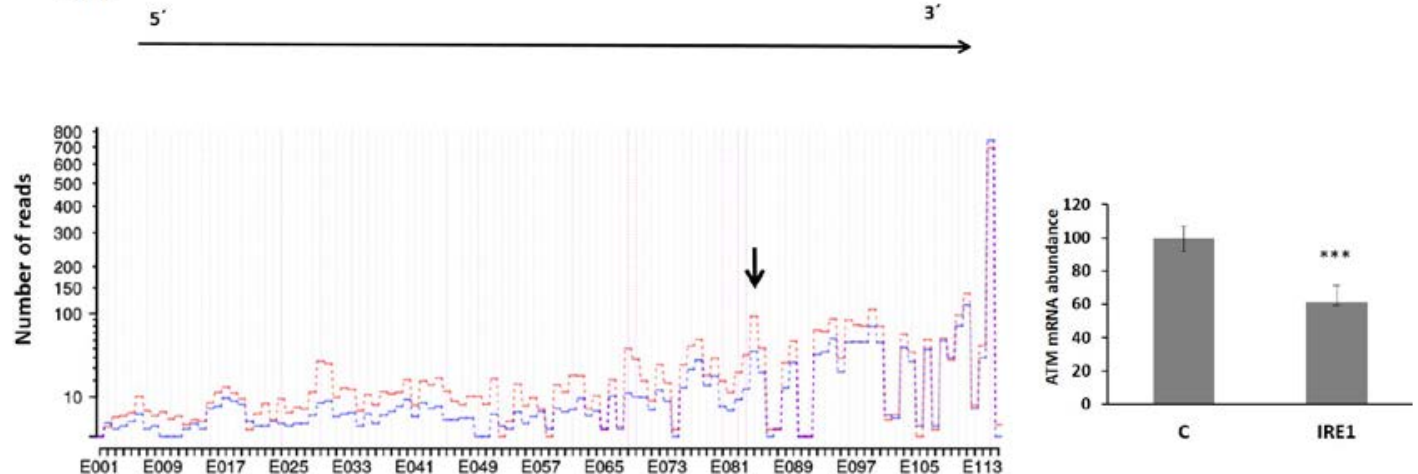

VPS13C
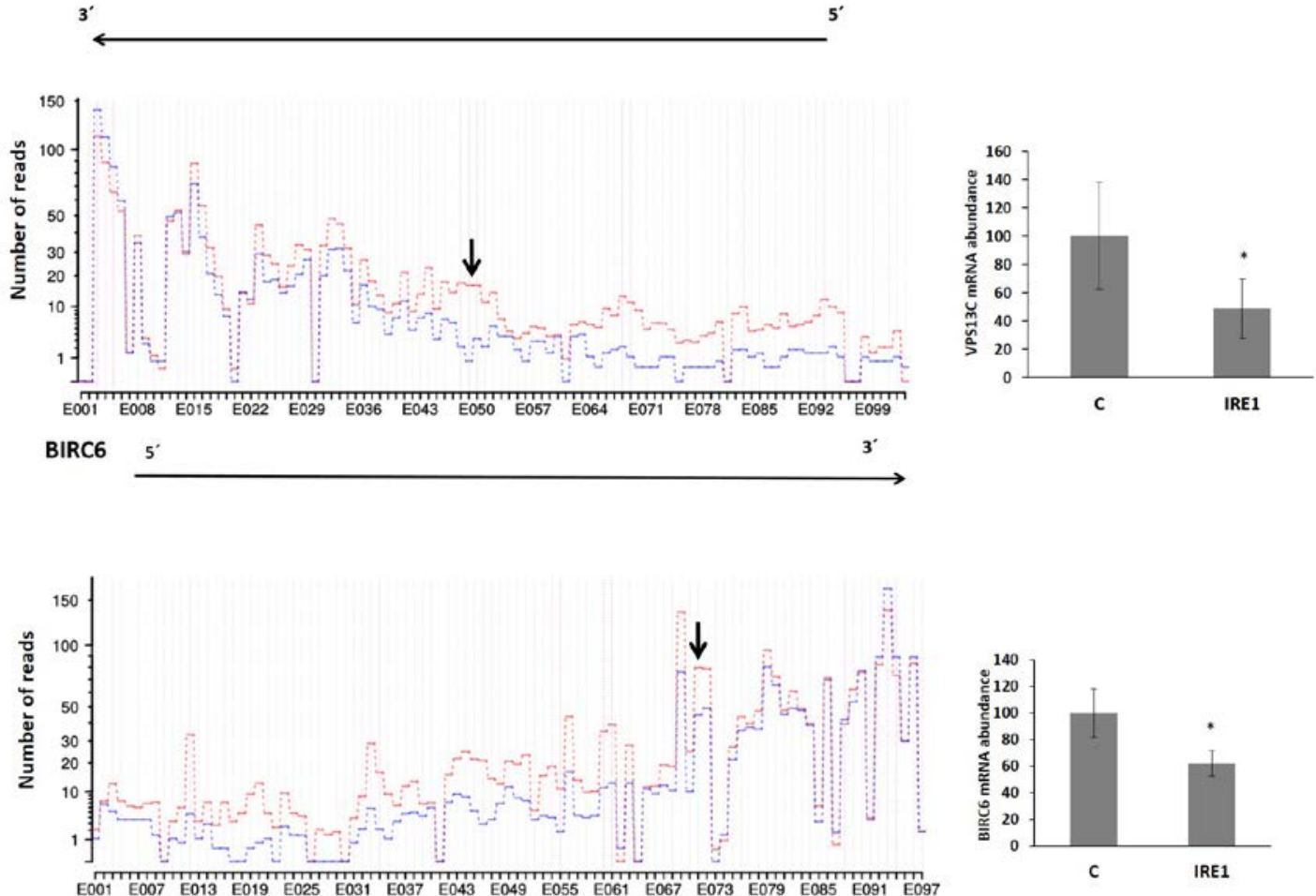

KMT2C
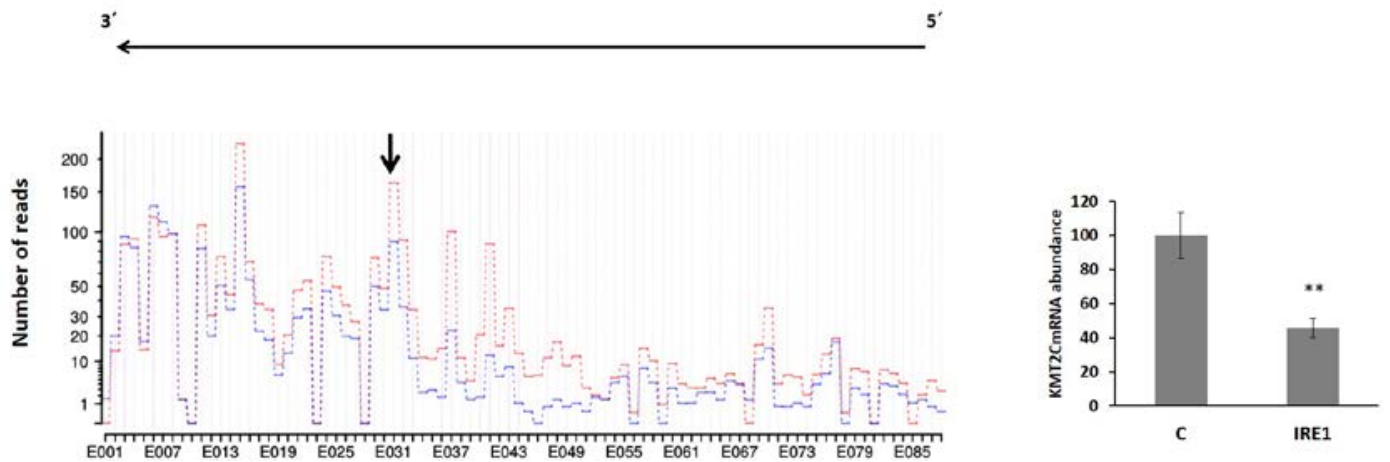
HUWE1

Figure S3
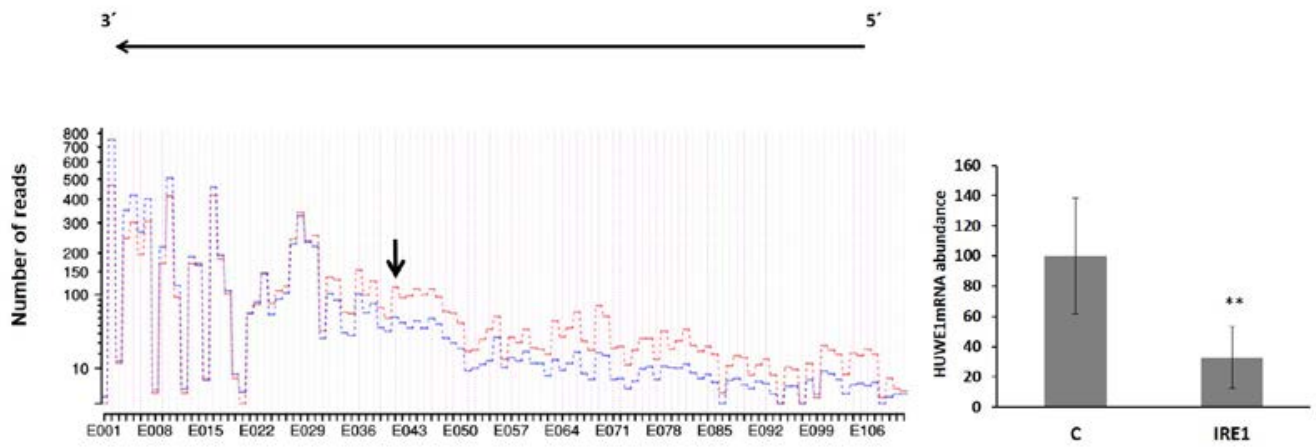

CDK12
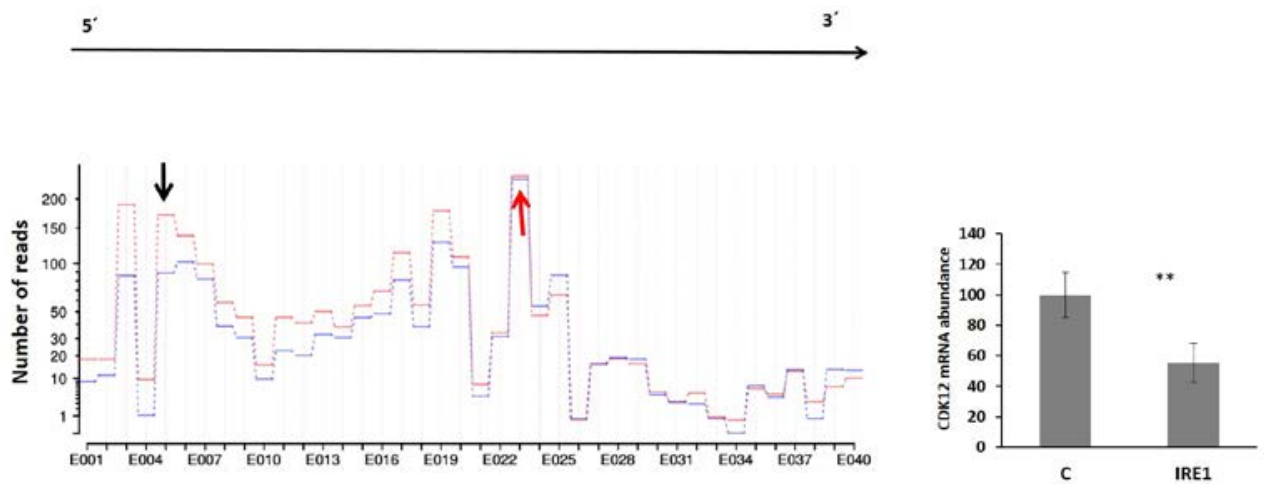

CENPF
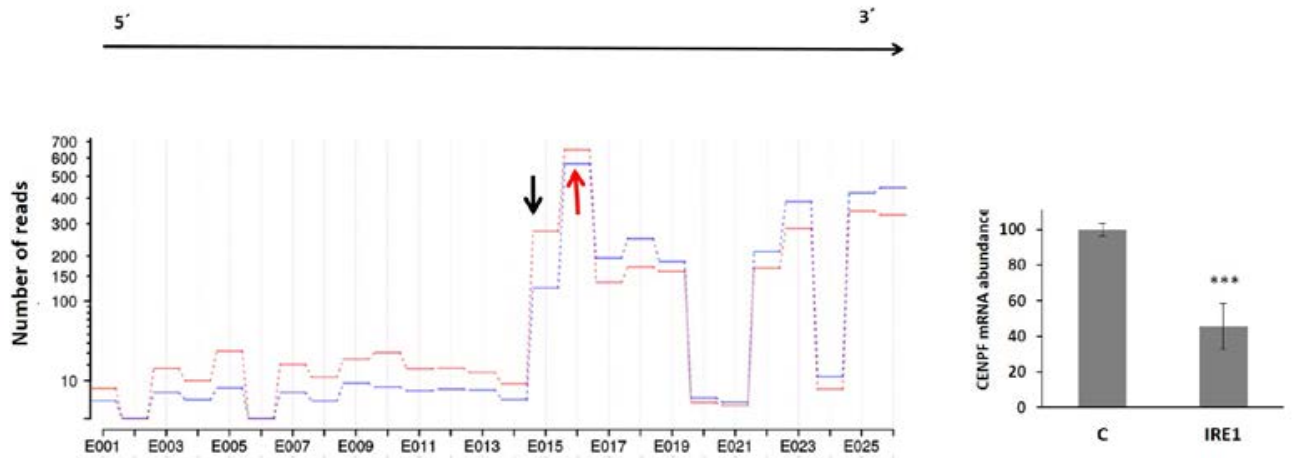

VPS13D
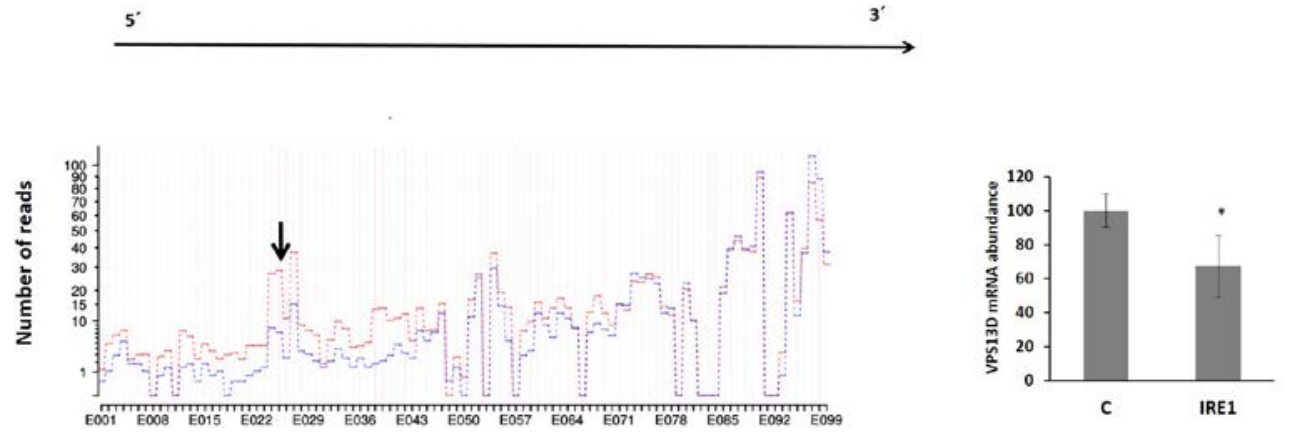
GOLGB1

Figure S3

$3^{\prime}$

$5^{\prime}$
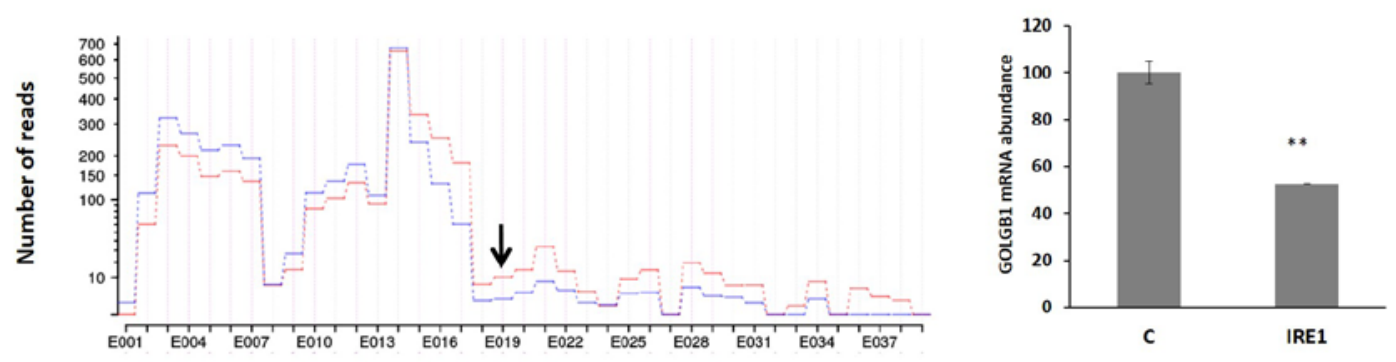

FAM168B
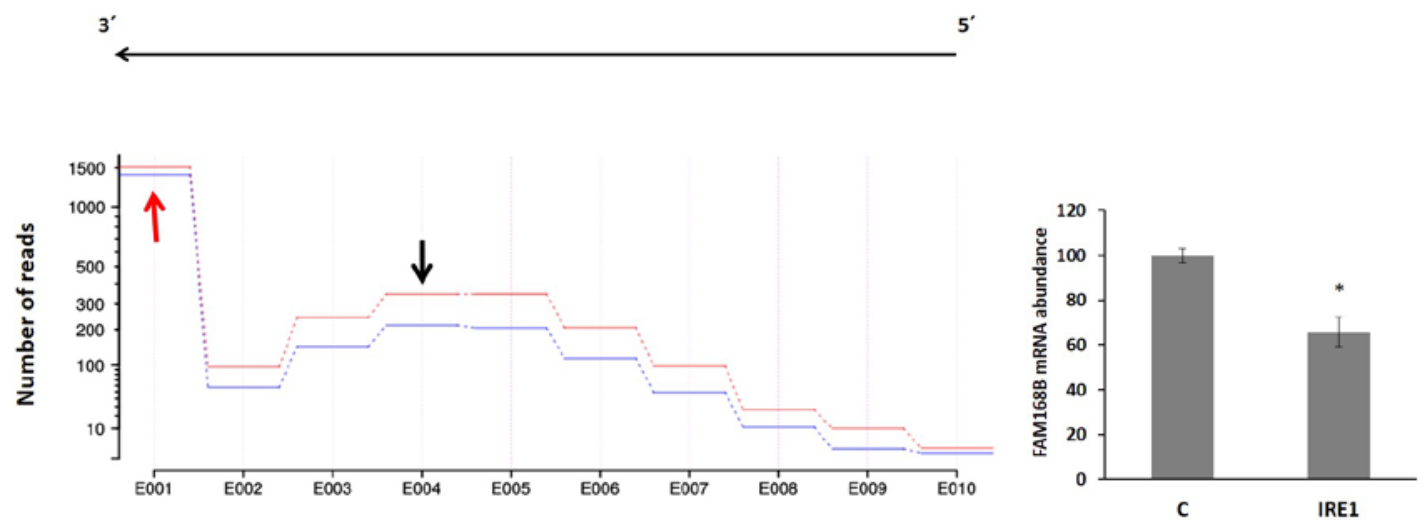

AKAP9
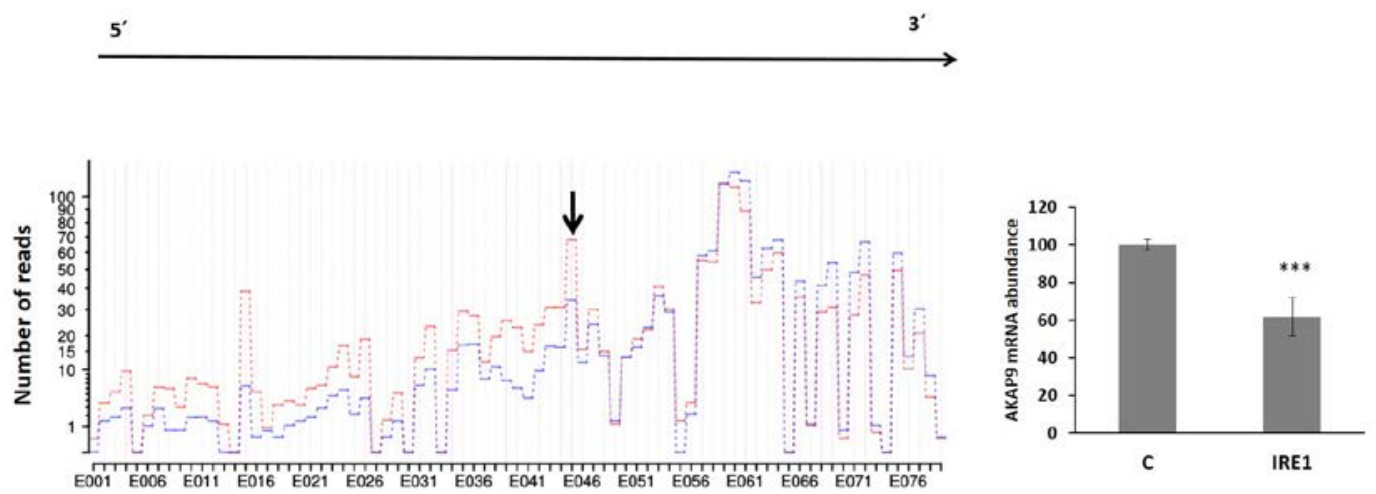

NOTCH1

3
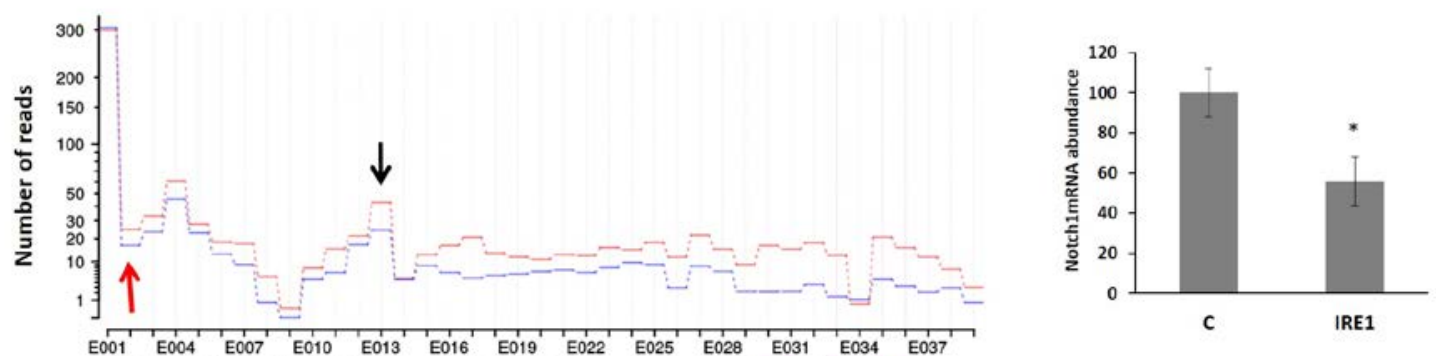
PCM1

Figure S3

$\stackrel{2}{5^{\prime}}$
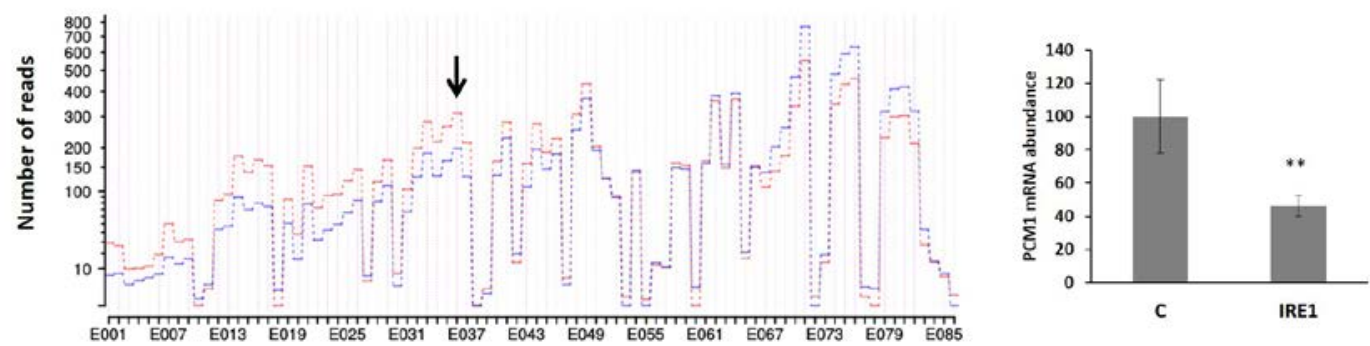

UBR2

5

3
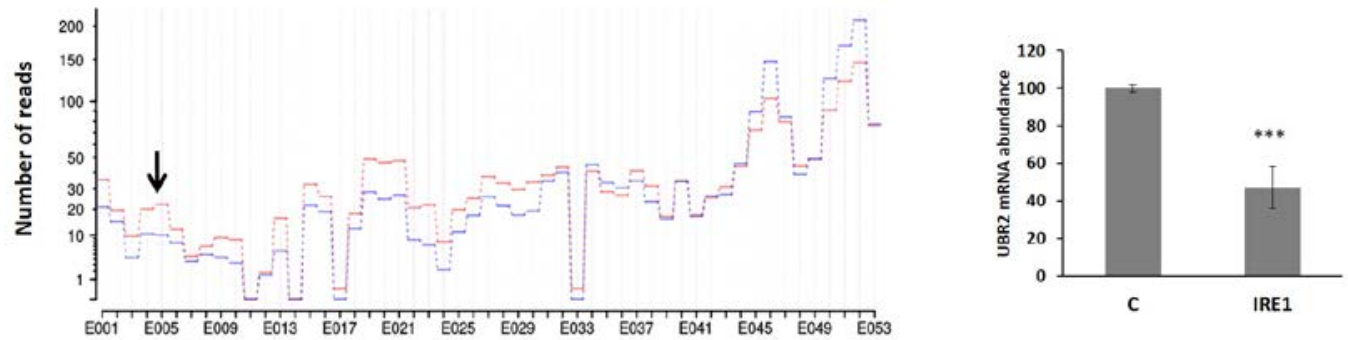

mTOR

$5^{\prime}$
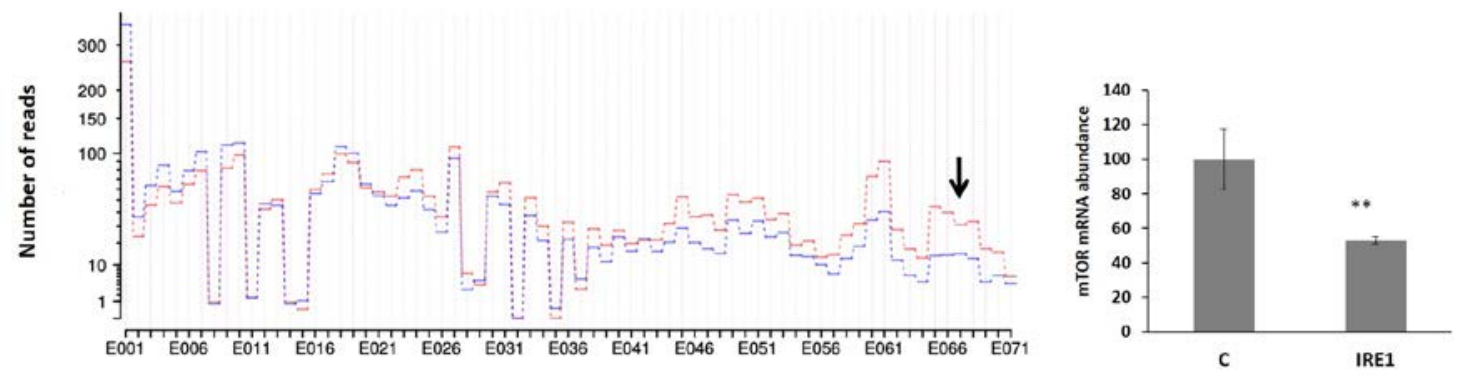

DICER

$3^{\prime}$

$5^{\prime}$
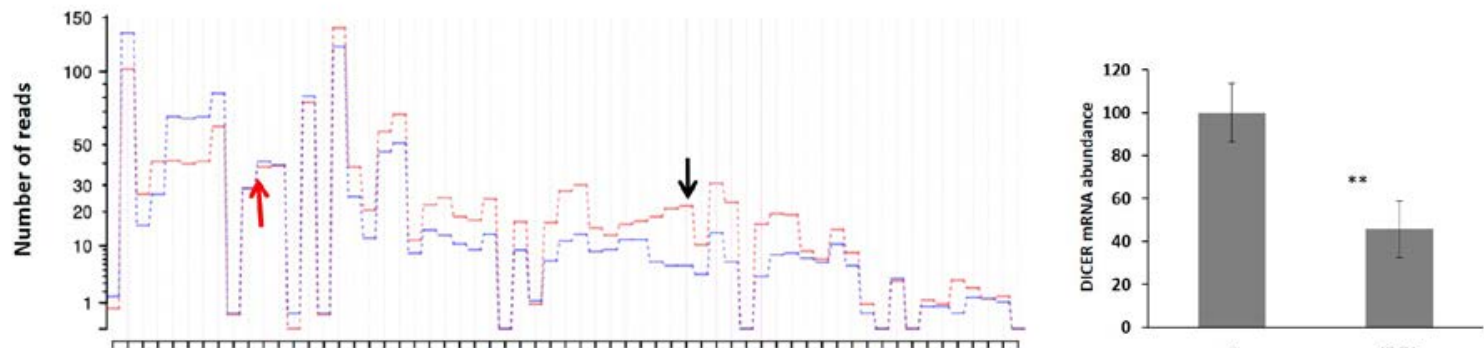
E001 E005 E009 E013 E017 E021 E025 E029 E033 E037 E041 E045 E049 E053 E057 E06

IRE1 
XRN1

Figure S3

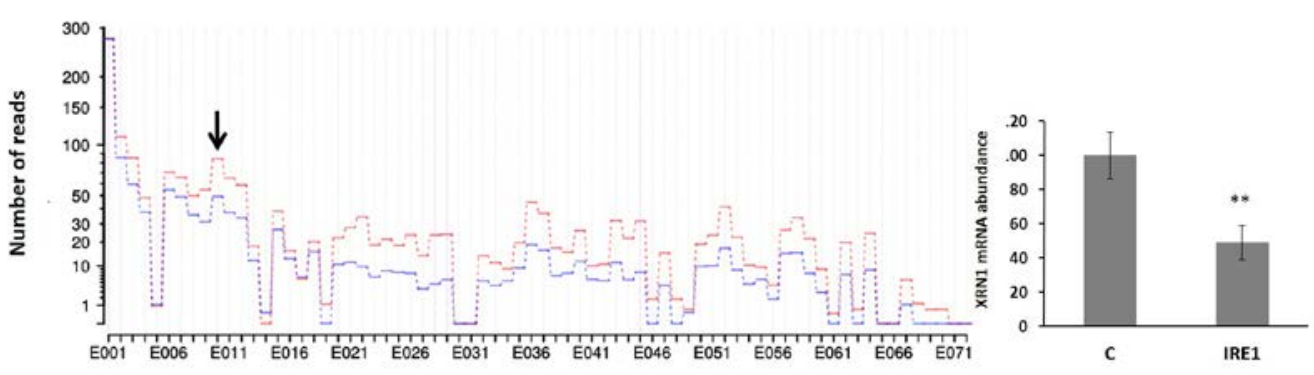

CUL9
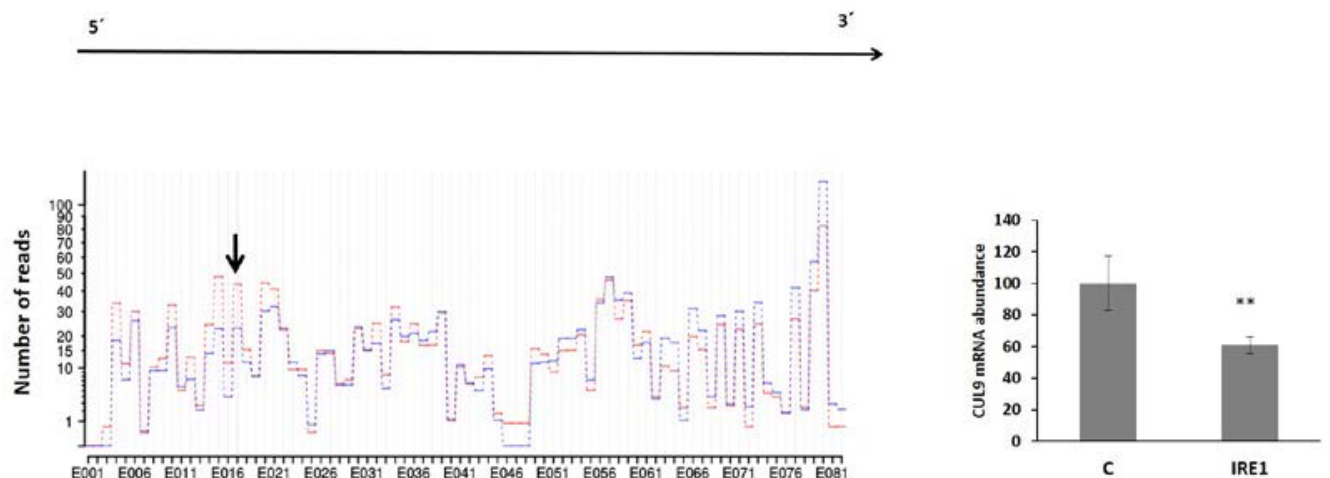

UBE4B

$5^{\prime}$

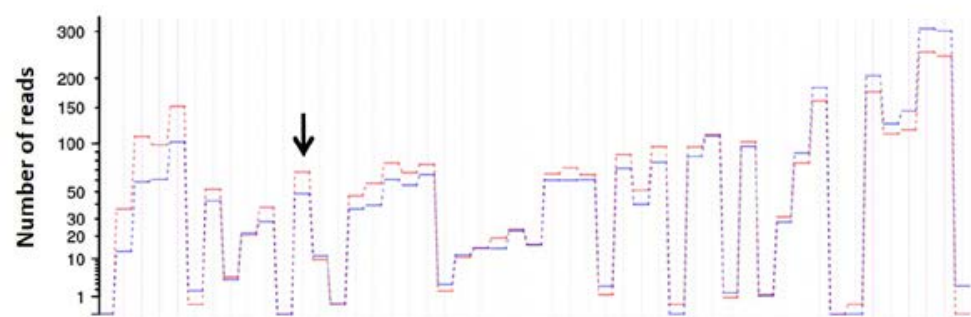

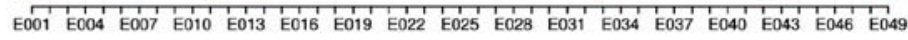

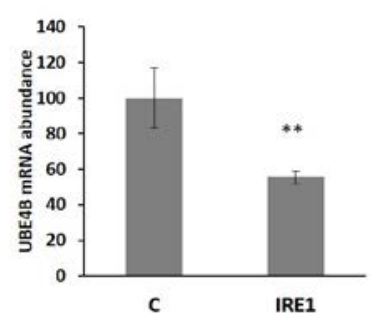

PSME4

3.
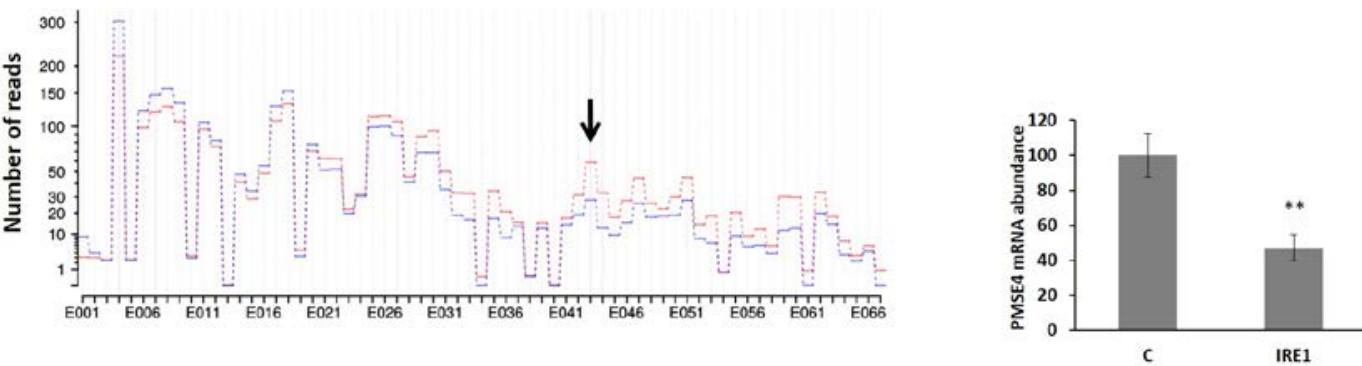


$$
3^{\prime}
$$
$5^{\prime}$

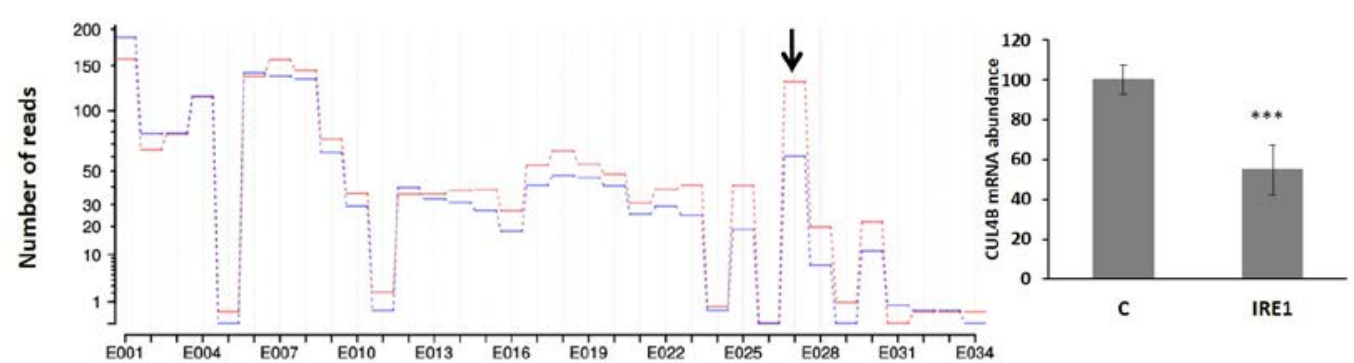

UBR3
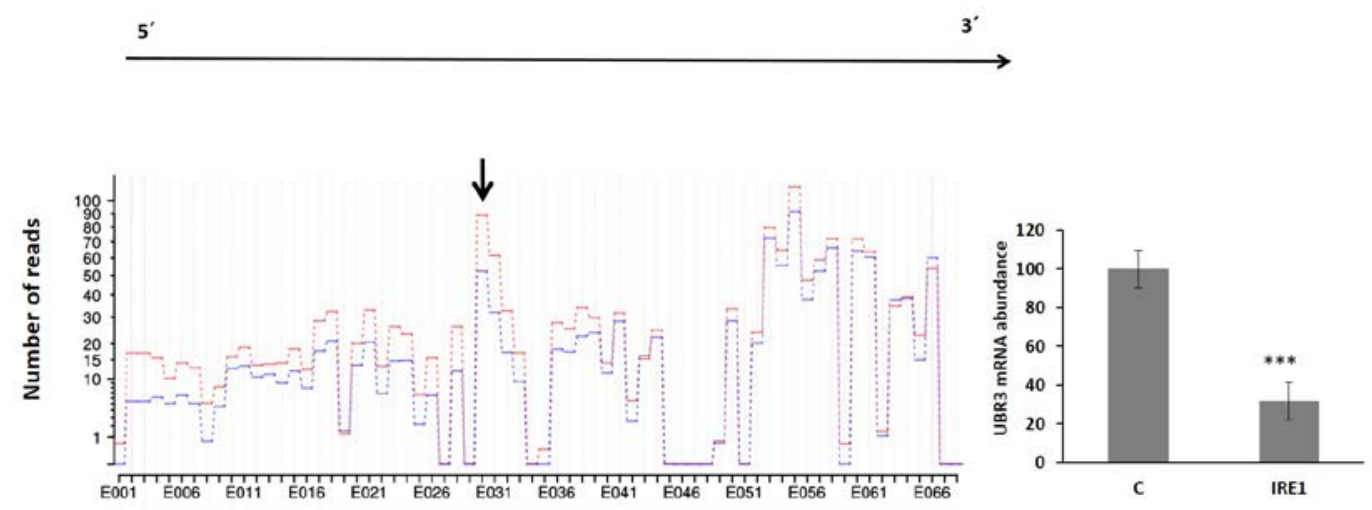

UBA6
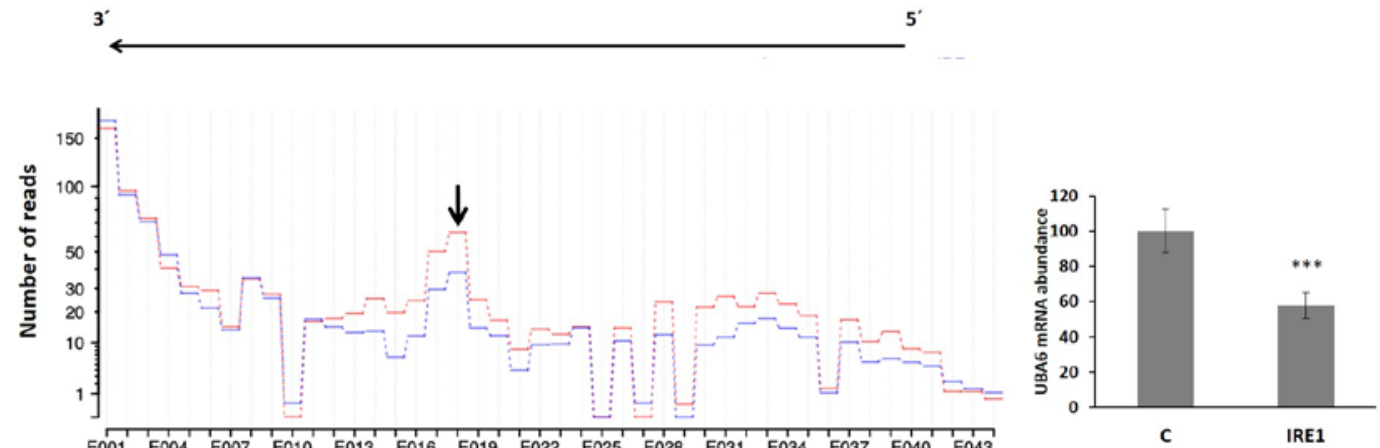

IKZF1
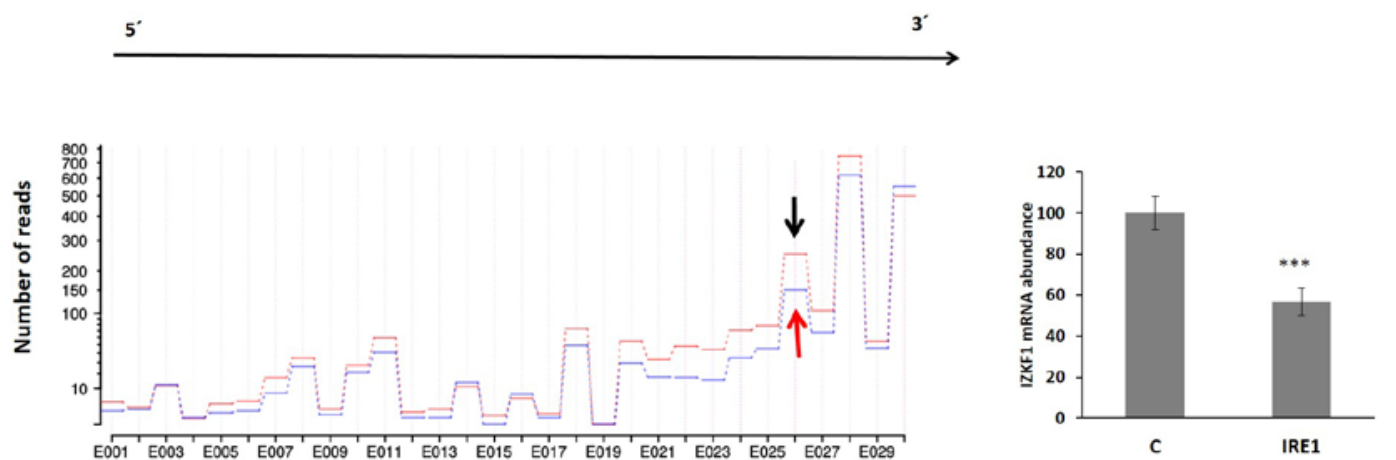
PSMD1

Figure S3
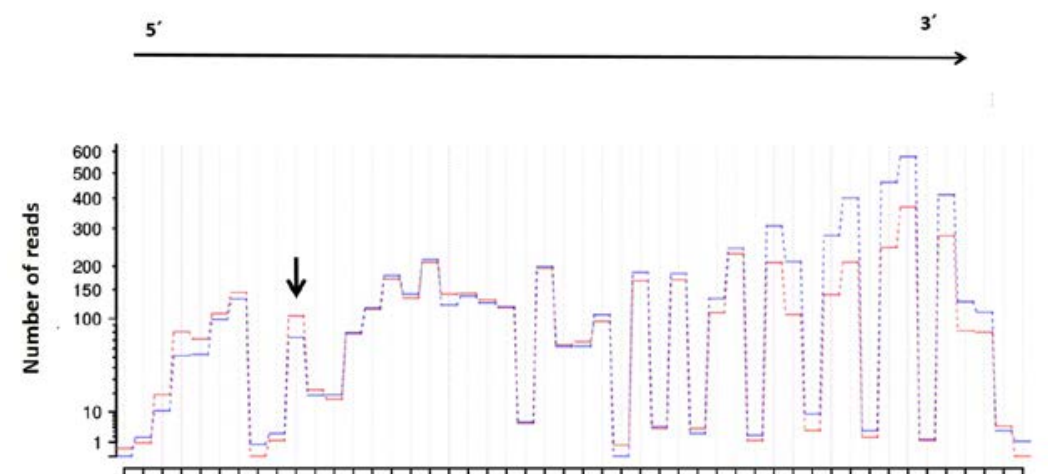
E001 E004 E007 E010 E013 E016 E019 E022 E025 E028 E031 E034 E037 E040 E043 E046

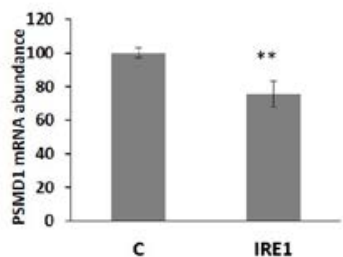

CUL5

$$
5
$$

$3^{\prime}$
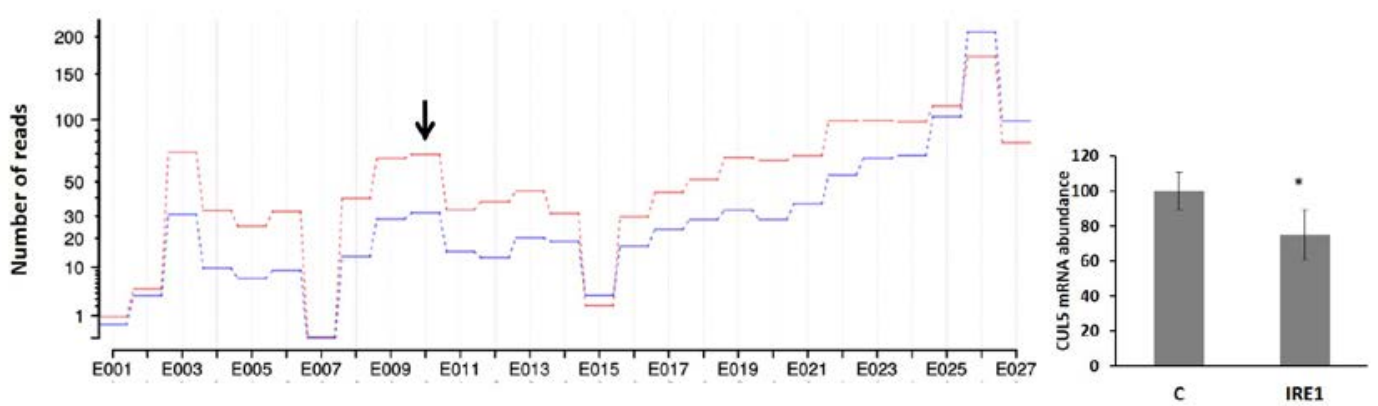

ERAP1
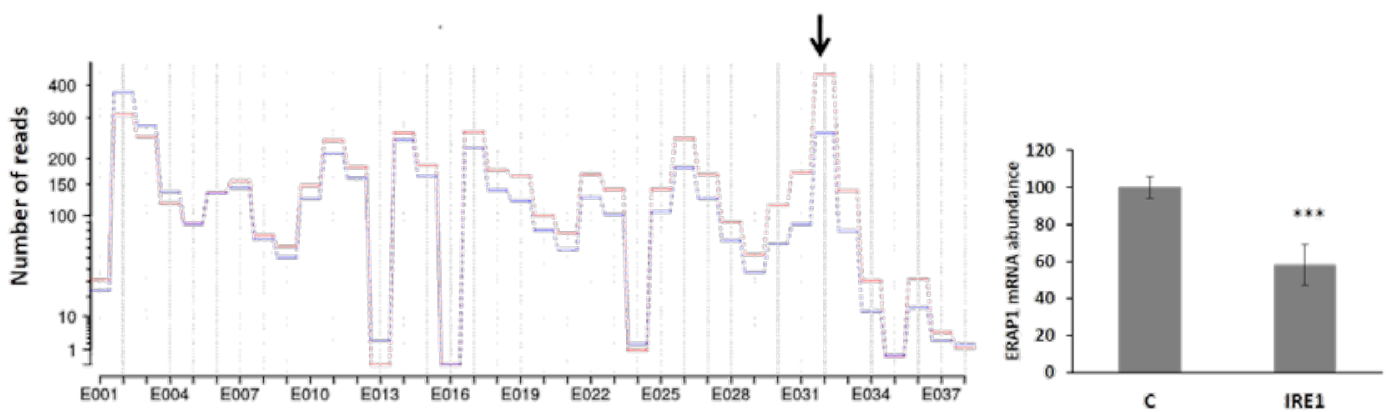

IRF4

5
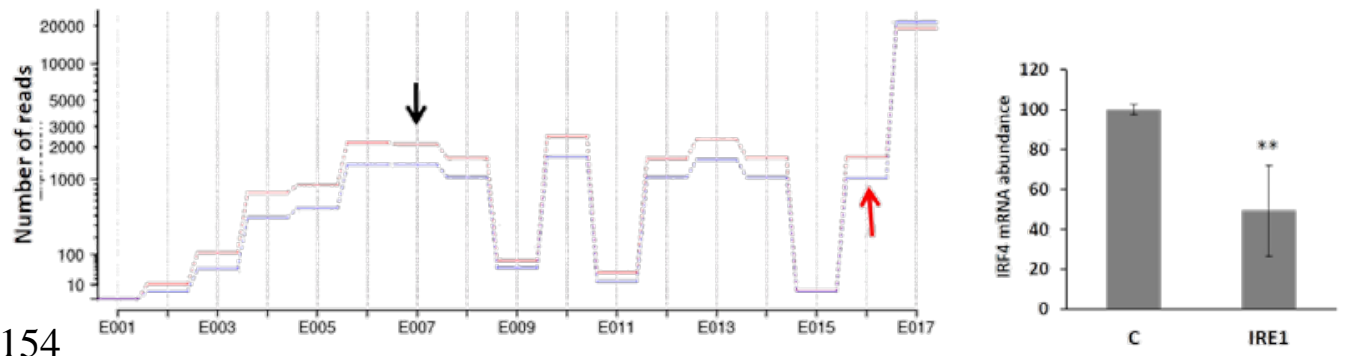
Figure S3. Validation of putative IRE1 substrates. Exon-usage plots of the 28 remaining putative mRNAs, showing the number of reads in mock (red) and IRE1-treated (blue) samples. The black arrows represent the site of primers used in the 5 ' region of the putative IRE1-substrates. Red arrows represent the site of primers mapping the predicted cleavage site. Right panel of each exon-usage plot shows the abundance of mRNA in the corresponding target. All results are presented as the means \pm SD of three experiments. $\left({ }^{*} p<0.05,{ }^{* *} p<0.01,{ }^{* * *} p<0.001\right)$. 

Conclusions 



\section{Chapter I:}

1- DEPTOR mRNA is overexpressed in plasma cells compared with B lymphocytes.

2- DEPTOR knockdown in myeloma cell lines induces dedifferentiation of myeloma cells, as demonstrated by the upregulation of PAX5 and BCL6, the downregulation of IRF4, and a clear reduction in cell size and endoplasmic reticulum mass.

3- MiR-135b and miR-642a regulate DEPTOR expression in MM and their upregulation results in myeloma cell dedifferentiation.

4- DEPTOR protein is differentially expressed among multiple myeloma patients. Progression free survival was significantly longer in MM patients with high DEPTOR expression than in those with low expression levels.

\section{Chapter II:}

5- IRE1 mRNA is overexpressed in plasma cells compared with B lymphocytes.

6- IRE1 is a direct target of miR-124, miR-506, miR-326, and miR-488.

7- IRE1 downregulation induced by shRNAs or ectopic transfection with miR-124 and miR506 lead to myeloma cell dedifferentiation.

\section{Chapter III:}

8- RNA sequencing shows that IRE1 RNase has a broad range of mRNA substrates in myeloma cells.

9- IRE1 regulates several proteins of key significance in multiple myeloma survival and proliferation.

10- The mRNAs of KLF13, DICER, NOTCH1, IRF4, ATR, BLIMP1, IKAROS, and CENPF genes are novel RIDD targets in multiple myeloma and the stress-induced degradation of these mRNAs is IRE1-dependent. 



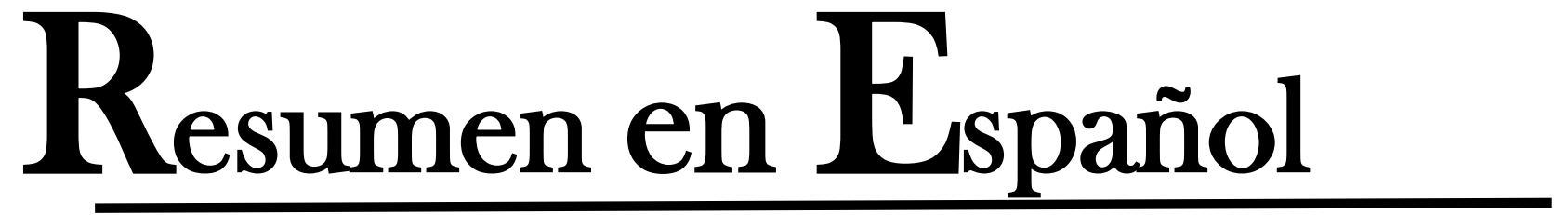





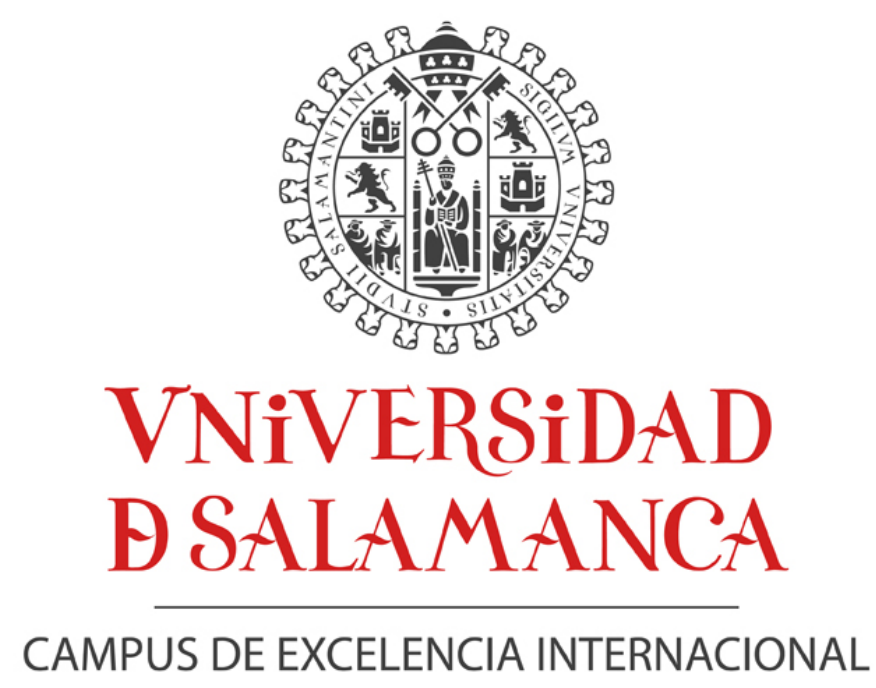

Facultad de Medicina

Departamento de Medicina

Hematología

Centro de Investigación del Cáncer (IBMCC-CSIC-USAL)

Nuevos conocimientos sobre la diferenciación de las células plasmáticas y la respuesta a las proteínas mal plegadas en el mieloma múltiple: papel de DEPTOR e IRE1

Tesis doctoral

Dalia Quwaider

Salamanca 



\section{Nuevos conocimientos sobre la diferenciación de las células plasmáticas y la respuesta a las proteínas mal plegadas en el mieloma múltiple: papel de DEPTOR e IRE1}

\section{Hipótesis}

La transición de células B (CB) a células plasmáticas (CP) implica profundas modificaciones transcripcionales que dan como resultado el fenotipo de CP, caracterizado por una capacidad de supervivencia a largo plazo, y la producción y secreción de anticuerpos. Los defectos en el desarrollo de las CP pueden provocar enfermedades graves. De hecho, la interrupción del proceso de diferenciación de la $\mathrm{CB}$, como consecuencia de mutaciones en factores clave en su maduración, se ha asociado con la transformación maligna de las CB.

Aunque el conocimiento de las bases moleculares que regulan la formación de la CP ha avanzado en los últimos años, muchos de los mecanismos implicados en el mantenimiento de las CP de larga duración, que son las células que se acumulan anormalmente en la medula ósea de los pacientes con MM, son aún desconocidos. Profundizar en el conocimiento de los factores implicados en la maduración de las CP podría ayudarnos a comprender la patogenia del MM y, por tanto, brindar nuevas perspectivas para el desarrollo de estrategias terapéuticas más efectivas que ayuden a bloquear la actividad tumoral de las CP en el MM.

En un análisis previo de expresión génica mediante microarrays, habíamos observado que el ARNm que codifica DEPTOR e IRE1 estaba sobreexpresado en las CP normales y en las células del mieloma múltiple (MM) en comparación con los linfocitos B normales. Por este motivo, planteamos la hipótesis de que la expresión variable de las proteínas DEPTOR e IRE1 podría contribuir a inducir diferentes estados de maduración de las células del MM. DEPTOR es un inhibidor de mTOR que está sobreexpresado en los MM con translocaciones de $C C N D 1$ o $C C N D 3$, o $M A F$ o $M A F B$. En estas células, se observó que se necesitaba una alta expresión de DEPTOR para mantener la activación de PI3K y AKT, y que la reducción de los niveles de DEPTOR conducía a la apoptosis. Sin embargo, no se ha descrito el posible papel de DEPTOR en la maduración de la CP. Se ha demostrado que el sensor de estrés en el retículo endoplásmico, IRE1, es necesario durante la diferenciación temprana y tardía de las CB. Por un lado, es fundamental en la etapa de células pro-B para la recombinación de los segmentos V(D)J de los genes de las inmunoglobulinas, y por otro lado, se ha demostrado que IRE1 es indispensable para la diferenciación terminal de la CB en CP a través de la activación de XBP1.

En los últimos años, se ha descubierto que numerosos microRNAs participan en la maduración de las CB. Este hecho nos llevó a pensar que la expresión tanto de DEPTOR 
como de IRE1 también podría estar regulada a nivel postranscripcional por microRNAs.

Además de participar en la maduración de la CP, también se ha demostrado que IRE1 es capaz de degradar algunos ARNm de una manera diferente a como lo hace con XBP1. Este mecanismo conocido como "regulated IRE1-dependent decay (RIDD)" puede depender de la naturaleza de los estímulos de estrés y del tejido donde se produzca. Diferentes investigaciones sugieren que aún quedan muchas dianas de RIDD por identificar. En las células de mieloma, la única diana de RIDD que se ha identificado es el ARNm de BLOC1S1. Nuestra hipótesis era que podrían existir muchas otras dianas RIDD en el MM y que algunas de ellas podrían regular la supervivencia o proliferación de las células del MM.

\section{Objetivos}

1) Investigar el papel del gen $D E P T O R$ en la diferenciación terminal de las células del mieloma múltiple.

- Estudiar los efectos del silenciamiento de DEPTOR en el estado de maduración de las células mielomatosas.

- Identificar microRNAs que modulen la expresión de DEPTOR en el MM y conocer su efecto sobre la maduración de la CP.

- Correlacionar el pronóstico de los pacientes con MM con los niveles de la proteína DEPTOR.

2) Descubrir la función de IRE1 en la maduración de las células del mieloma múltiple.

- Analizar el efecto del silenciamiento de IRE1 en el estado de maduración de las células del mieloma.

- Explorar la regulación epigenética de IRE1 por microRNAS.

3) Identificar dianas de "regulated IRE1-dependent decay (RIDD)" en el MM mediante la secuenciación del ARN.

- Analizar la presencia del sitio de escisión de IRE1 en las dianas de RIDD.

- Investigar si las dianas identificadas se degradan tras la inducción del estrés en el retículo endoplásmico. 


\section{Resultados}

\section{Capítulo I:}

En este capítulo, exploramos el papel de DEPTOR, un inhibidor de mTOR, en la diferenciación terminal de las células del mieloma múltiple (MM) y su repercusión potencial en la supervivencia de los pacientes. El silenciamiento de DEPTOR en las líneas celulares H929 y MM1S indujo la desdiferenciación de las células mielomatosas, como lo demostró el aumento de la expresión de los factores de transcripción PAX5 y BCL6, el descenso de la expresión del factor de transcripción IRF4, y la reducción en el tamaño celular y del retículo endoplásmico. Este efecto fue independiente de la señalización de mTOR, ya que los sustratos de mTOR no se vieron afectados por el descenso de DEPTOR. Además, se investigó la posibilidad de que DEPTOR estuviera desregulado en el MM por la acción de los miRNAs. Así, se observó que la expresión ectópica de los miR-135b y miR-642a en líneas celulares de MM disminuyó sustancialmente los niveles de la proteína DEPTOR y provocó la desdiferenciación de las células tumorales del MM.

La cuantificación de la proteína DEPTOR en pacientes con MM puso de manifiesto que sus altos niveles de expresión se asociaban con una supervivencia libre de progresión más prolongada. Nuestros resultados demuestran por primera vez que la expresión de DEPTOR es requerida para mantener la diferenciación de las células del MM y que un alto nivel de su expresión se asocia con una supervivencia más prolongda.

\section{Capítulo II:}

La maduración de las células B conlleva una serie de procesos estrechamente controlados por determinadas vías de señalización, factores de transcripción y miRNAs. En este capítulo, investigamos el papel de IRE1 en la diferenciación terminal de las células del MM. El silenciamiento de IRE1 en las líneas celulares H929 y MM1S provocó la desdiferenciación de las células mielomatosas, como lo evidenció el aumento de expresión de PAX5 y BCL6. Además, los ensayos de luciferasa y de ganancia de función demostraron que los miR-124 y miR-506 regulaban la expresión de IRE1 en el MM. La expresión ectópica de miR-124 y miR506 en las líneas celulares de mieloma disminuyó sustancialmente los niveles de proteína IRE1 y causó la desdiferenciación de las células de mieloma, como lo 
demostró la disminución de la concentración de IRF4 e IgK, y la reducción en el tamaño celular y del retículo endoplásmico. Nuestros resultados muestran que la expresión de IRE1 consigue mantener la diferenciación de las células tumorales del MM.

\section{Capítulo III:}

IRE1 es un sensor de la respuesta a las proteínas mal plegadas con actividad de quinasa y endonucleasa. Desempeña un papel central en la respuesta al estrés en el retículo endoplásmico (RE) a través del "splicing" no convencional del ARNm de XBP1 y del proceso "regulated IRE1-dependent decay (RIDD)". Se sabe que las células tumorales del MM poseen un nivel elevado de estrés basal en el RE, sin embargo, la actividad de RIDD no ha sido bien estudiada en esta enfermedad. Para investigar nuevas dianas de RIDD de posible relevancia para la supervivencia y/o proliferación de las células del MM, se combinaron el ensayo de escisión in vitro con técnicas de secuenciación de ARN. El análisis bioinformático reveló cientos de posibles sustratos de IRE1, de los cuales 32 fueron elegidos para la validación. Analizando la estructura secundaria de los sustratos de IRE1, se observó que las secuencias consenso de los mRNA de IRF4, PRDM1, IKZF1, KLF13, NOTCH1, ATR, DICER, RICTOR, CDK12, FAM168B y CENPF estaban acompañadas por una estructura "stem loop" esencial para la escisión mediada por IRE1. Además los niveles de ARNm y proteínas correspondientes a estas dianas se atenuaron de manera dependiente de IRE1 mediante el tratamiento con agentes inductores del estrés en el RE. Nuestros resultados demuestran por primera vez que IRE1 es un regulador clave de varias proteínas implicadas en la supervivencia y proliferación del MM. 


\section{Conclusiones}

\section{Capítulo I:}

1- El ARNm de DEPTOR está sobreexpresado en las células plasmáticas en comparación con los linfocitos B.

2- El silenciamiento de DEPTOR en las líneas celulares de mieloma múltiple induce la desdiferenciación de las células mielomatosas, como lo demuestra el aumento de la expresión de PAX5 y BCL6, el descenso de la expresión de IRF4 y una clara reducción del tamaño celular y del retículo endoplásmico.

3- Los miR-135b y miR-642a regulan la expresión de DEPTOR en MM y su sobreexpresión da como resultado la desdiferenciación de las células del mieloma.

4- La proteína DEPTOR se expresa de forma diferencial entre los pacientes con mieloma múltiple. La supervivencia libre de progresión fue significativamente más larga en pacientes con MM con alta expresión de DEPTOR que en aquellos con bajos niveles de expresión.

\section{Capitulo II:}

5- El ARNm de IRE1 está sobreexpresado en las células plasmáticas en comparación con los linfocitos B.

6- IRE1 es una diana directa de los miR-124, miR-506, miR-326 y miR-488.

7- El silenciamiento de IRE1 inducido por shRNA o la transfección ectópica con miR-124 y miR-506 conduce a la desdiferenciación de las células del mieloma.

\section{Capítulo III:}

8- La secuenciación del ARN muestra que la ARNasa IRE1 tiene una amplia gama de sustratos de ARNm en las células del mieloma.

9- IRE1 regula varias proteínas de importancia clave en la supervivencia y proliferación del mieloma múltiple.

10- Los ARNm de los genes KLF13, DICER, NOTCH1, IRF4, ATR, BLIMP1, IKAROS y CENPF son nuevas dianas de RIDD en el mieloma múltiple y la degradación de estos ARNm inducida por el estrés depende de IRE1. 

Anexo 



\section{Scientific contribution during the thesis:}

1) Herrero AB, Quwaider D, Corchete LA, Mateos MV, García-Sanz R, Gutiérrez NC. FAM46C controls antibody production by the polyadenylation of immunoglobulin mRNAs and inhibits cell migration in multiple myeloma. J Cell Mol Med. 2020. Apr;24(7):4171-4182. doi: 10.1111/jcmm.15078. Epub 2020 Mar 6. PMID: 32141701; PMCID: PMC7171423.

2) Misiewicz-Krzeminska I, Krzeminski P, Corchete LA, Quwaider D, Rojas EA, Herrero AB, Gutiérrez NC. Factors regulating microRNA Expression and Function in Multiple Myeloma. Noncoding RNA. 2019 Jan 16; 5(1):9. doi: 10.3390/ncrna5010009. PMID: 30654527; PMCID: PMC6468559.

3) Quwaider D, Corchete LA, Misiewicz-Krzeminska I, Sarasquete ME, Pérez JJ, Krzeminski P, Puig N, Mateos MV, García-Sanz R, Herrero AB, Gutiérrez NC. DEPTOR maintains plasma cell differentiation and favorably affects prognosis in multiple myeloma. J Hematol Oncol. 2017 Apr 18; 10(1):92. doi: 10.1186/s13045-017-04618. PMID: 28420429; PMCID: PMC5395780.

4) Quijada-Álamo M, Hernández-Sánchez M, Robledo C, Hernández-Sánchez JM, Benito R, Montaño A, Rodríguez-Vicente AE, Quwaider D, Martín AÁ, García-Álvarez M, Vidal-Manceñido MJ, Ferrer-Garrido G, Delgado-Beltrán MP, Galende J, Rodríguez JN, Martín-Núñez G, Alonso JM, García de Coca A, Queizán JA, Sierra M, Aguilar C, Kohlmann A, Hernández JÁ, González M, Hernández-Rivas JM. Next-generation sequencing and FISH studies reveal the appearance of gene mutations and chromosomal abnormalities in hematopoietic progenitors in chronic lymphocytic leukemia. J Hematol Oncol. 2017 Apr 11;10(1):83. doi: 10.1186/s13045-017-0450y. PMID: 28399885; PMCID: PMC5387353.

5) López-Iglesias AA, Herrero AB, Chesi M, San-Segundo L, González-Méndez L, Hernández-García S, Misiewicz-Krzeminska I, Quwaider D, Martín-Sánchez M, Primo D, Paíno T, Bergsagel PL, Mehrling T, González-Díaz M, San-Miguel JF, Mateos MV, Gutiérrez NC, Garayoa M, Ocio EM. Preclinical anti-myeloma activity of EDOS101, a new bendamustine-derived molecule with added HDACi activity, through potent DNA damage induction and impairment of DNA repair. J Hematol Oncol. 2017 Jun 20;10(1):127. doi: 10.1186/s13045-017-0495-y. PMID: 28633670; PMCID: PMC5477689. 
6) Misiewicz-Krzeminska I, Sarasquete ME, Vicente-Dueñas C, Krzeminski P, Wiktorska K, Corchete LA, Quwaider D, Rojas EA, Corral R, Martín AA, Escalante F, Bárez A, García JL, Sánchez-García I, García-Sanz R, San Miguel JF, Gutiérrez NC. Posttranscriptional Modifications Contribute to the Upregulation of CyclinD2 in Multiple Myeloma. Clin Cancer Res. 2016 Jan 1;22(1):207-17. doi:10.1158/1078-0432.CCR14-2796. Epub 2015 Sep 4. PMID: 26341922.

7) Rodríguez-Vicente AE, Quwaider D, Benito R, Misiewicz-Krzeminska I, HernándezSánchez M, de Coca AG, Fisac R, Alonso JM, Zato C, de Paz JF, García JL, Sarasquete ME, Hernández JÁ, Corchado JM, González M, Gutiérrez NC, Hernández-Rivas JM. MicroRNA-223 is a novel negative regulator of HSP90B1 in CLL. BMC Cancer. 2015 Apr 8;15:238. doi: 10.1186/s12885-015-1212-2. PMID: 25880332; PMCID: PMC4404064.

8) Garcia-Gomez A, Quwaider D, Canavese M, Ocio EM, Tian Z, Blanco JF, Berger AJ, Ortiz-de-Solorzano C, Hernández-Iglesias T, Martens AC, Groen RW, Mateo-Urdiales J, Fraile S, Galarraga M, Chauhan D, San Miguel JF, Raje N, Garayoa M. Preclinical activity of the oral proteasome inhibitor MLN9708 in Myeloma bone disease. Clin Cancer Res. 2014 Mar 15;20(6):1542-54. doi: 10.1158/1078-0432.CCR-13-1657. Epub 2014 Jan 31. PMID: 24486586.

9) Misiewicz-Krzeminska I, Sarasquete ME, Quwaider D, Krzeminski P, Ticona FV, Paíno T, Delgado M, Aires A, Ocio EM, García-Sanz R, San Miguel JF, Gutiérrez NC. Restoration of microRNA-214 expression reduces growth of myeloma cells through positive regulation of P53 and inhibition of DNA replication. Haematologica. 2013 Apr;98(4):640-8. doi: 10.3324/haematol.2012.070011. Epub 2012 Oct 25. PMID: 23100276 ; PMCID: PMC3659997.

\section{Submitted papers}

Quwaider D, Corchete LA, Martín-Izquierdo M, Hernández-Sánchez JM, Rojas EA, García-Sanz R, Herrero AB, Gutiérrez NC. RNA sequencing identifies novel regulated IRE1-dependent decay targets that affect multiple myeloma survival and proliferation. 


\title{
DEPTOR maintains plasma cell differentiation and favorably affects prognosis in multiple myeloma
}

\author{
Dalia Quwaider ${ }^{1,3}$, Luis A. Corchete ${ }^{1,3}$, Irena Misiewicz-Krzeminska ${ }^{1,3,4}$, María E. Sarasquete ${ }^{1,3}$, José J. Pérez², \\ Patryk Krzeminski ${ }^{1,3}$, Noemí Puig ${ }^{1,2,3}$, María Victoria Mateos ${ }^{1,2,3}$, Ramón García-Sanz ${ }^{1,2,3}$, Ana B. Herrero ${ }^{1,3+}$ \\ and Norma C. Gutiérrez ${ }^{1,2,3^{*}+}$
}

\begin{abstract}
Background: The B cell maturation process involves multiple steps, which are controlled by relevant pathways and transcription factors. The understanding of the final stages of plasma cell (PC) differentiation could provide new insights for therapeutic strategies in multiple myeloma (MM). Here, we explore the role of DEPTOR, an mTOR inhibitor, in the terminal differentiation of myeloma cells, and its potential impact on patient survival.

Methods: The expression level of DEPTOR in MM cell lines and B cell populations was measured by real-time RT-PCR, and/or Western blot analysis. DEPTOR protein level in MM patients was quantified by capillary electrophoresis immunoassay. RNA interference was used to downregulate DEPTOR in MM cell lines.

Results: DEPTOR knockdown in H929 and MM1S cell lines induced dedifferentiation of myeloma cells, as demonstrated by the upregulation of PAX5 and BCL6, the downregulation of IRF4, and a clear reduction in cell size and endoplasmic reticulum mass. This effect seemed to be independent of mTOR signaling, since mTOR substrates were not affected by DEPTOR knockdown. Additionally, the potential for DEPTOR to be deregulated in MM by particular miRNAs was investigated. The ectopic expression of miR-135b and miR-642a in myeloma cell lines substantially diminished DEPTOR protein levels, and caused dedifferentiation of myeloma cells. Interestingly, the level of expression of DEPTOR protein in myeloma patients was highly variable, the highest levels being associated with longer progression-free survival.
\end{abstract}

Conclusions: Our results demonstrate for the first time that DEPTOR expression is required to maintain myeloma cell differentiation and that high level of its expression are associated with better outcome.

Primary samples used in this study correspond to patients entered into GEM2010 trial (registered at www. clinicaltrials.gov as \#NCT01237249, 4 November 2010).

Keywords: DEPTOR, Multiple myeloma, Plasma cell development, B lymphocyte differentiation, miRNAs

\section{Background}

Multiple myeloma (MM) is a clonal disorder of B cells $(\mathrm{BCs})$ in the final stage of differentiation that accounts for approximately $10 \%$ of all hematological cancers [1]. MM is characterized by clonal accumulation of malignant plasma cells (PCs) in the bone marrow, which secrete a

\footnotetext{
* Correspondence: normagu@usal.es

${ }^{\dagger}$ Equal contributors

${ }^{1}$ Cancer Research Center-IBMCC (USAL-CSIC), Salamanca, Spain

${ }^{2}$ Department of Hematology, University Hospital of Salamanca, Paseo San

Vicente, 58-182, Salamanca 37007, Spain

Full list of author information is available at the end of the article
}

monoclonal immunoglobulin. Although several therapeutic agents are available, MM remains incurable. Knowledge of all the factors involved in PC differentiation could provide new insights of relevance to therapeutic strategies for MM. In fact, in some hematological neoplasms, the malignant transformation of $\mathrm{BC}$ has been associated with the disruption of the $\mathrm{B}$ cell differentiation process, such as mutations of certain key BC maturation factors [2-4]. The transition from B lymphoid precursors to antibody-secreting PCs involves several molecular and cellular modifications including transcriptional changes, expansion of the cytoplasm 
and the secretory organelles to accommodate high-rate synthesis of immunoglobulins, unfolded protein response (UPR) activation, and changes in cell surface antigen expression [5-8]. It has been demonstrated that the transcriptomes of $\mathrm{BC}$ and $\mathrm{PC}$ are maintained by two groups of transcriptional factors: those that promote the $\mathrm{B}$ cell program, such as PAX5, BCL6, and $\mathrm{BACH}$, and those that favor and facilitate PC differentiation, notably IRF4, BLIMP1, and XBP1 [9]. Interestingly, many of these transcription factors repress others required for the alternative developmental state, thereby establishing mutually exclusive gene expression programs [9-12]. Besides transcriptional factors, other types of proteins and biochemical pathways could be involved in the transformation of $\mathrm{BC}$ into mature PC.

Using microarray expression data, we found that the mRNA-encoding DEPTOR, an inhibitor of mTORC1 and mTORC2 kinases activities [13], was overexpressed in normal PCs (NPCs) and myeloma cells compared with normal B lymphocytes (NBLs) [14], which raised the possibility that this protein contributes to PC differentiation. The complete role of DEPTOR within the cells has not yet been fully elucidated, although the involvement of DEPTOR in several biological processes, such as cell growth, apoptosis, and autophagy, has been reported [15]. A potential role of DEPTOR as a tumor suppressor or as an oncogene, depending on cell context, has also been described. It is considered a tumor suppressor, functioning by the inhibition of mTOR, whose activity is frequently hyperactivated in many human tumors. Indeed, DEPTOR has been found to be downregulated in many types of human cancers. However, it is also overexpressed in many other tumor types, including chronic myeloid leukemia, and MM [13, 16]. The overexpression of DEPTOR in MM has been associated with translocations involving MAF transcription factors and CCND1 and CCND3 genes [13]. Furthermore, DEPTOR seems to be overexpressed in MM with copy number gains of $8 \mathrm{q} 24$ where DEPTOR is located [17].

Here, we report for the first time that DEPTOR maintains the terminal differentiation of MM cells. Knockdown of DEPTOR reverts the transcriptional program of the $\mathrm{PC}$ to that characteristic of a $\mathrm{BC}$. In addition, we found that microRNA deregulation in MM, specifically miR642a and miR135b downregulation, may also underpin the overexpression of DEPTOR.

\section{Methods}

\section{Cell lines and primary samples}

The human multiple myeloma cell lines (MMCL), NCIH929, MM1S, and U266 were acquired from the ATCC (American Type Culture Collection), and the JJN3, RPMI8226, OPM-2, KMS12BM, KMS12PE, and HEK923 lines were obtained from the Deutsche Sammlung von Mikroorganismen and Zellkulturen (DSMZ). Cell line identity was confirmed periodically by STR analysis with the PowerPlex 16 HS system kit (www.promega.com) and online STR matching analysis (www.dsmz.de/fp/cgi-bin/ str.html). Cell lines were cultured in RPMI 1640 medium supplemented with $10 \%$ fetal bovine serum and antibiotics (Gibco Life Technologies, Grand Island, NY, USA). Bone marrow (BM) samples from ten healthy donors were sorted by a FACSAria equipment into four $\mathrm{BC}$ populations: immature B cells (CD34-, CD19 +, CD10+, CD38+ +), naive B cells (CD19+, CD27-, CD10-), memory B cells (CD19+, CD138-, CD27+, CD38+), and plasma cells (CD38+++, CD138+, CD45low). Monoclonal antibodies were purchased as follows: anti-CD45-FITC (clone D3/9) and anti-CD19-PECy7 (clone A3-B1) from Immunostep (Salamanca, Spain); anti-CD38-PerCP-Cy ${ }^{\mathrm{mm}} 5.5$ (clone HIT2), anti-CD34-APC (clone 8G12), and anti-CD27BV421 (clone M-T271) from BD Biosciences (San Jose, CA, USA); anti- CD138-Pacific OrangeTM (clone B-A38) from Exbio Praha (Vestec, Czech Republic); and antiCD10-PE (clone ALB1) from Beckman Coulter (Pasadena, CA, USA). CD138+ plasma cells were isolated from BM samples of 24 patients with newly diagnosed MM included in the GEM2010 Spanish trial (bortezomib, melphalan, and prednisone plus lenalidomide and dexamethasone), using an autoMACS separation system (Miltenyi-Biotec, Auburn, CA, USA).

\section{RNA extraction and quantitative real-time PCR analysis}

RNA was extracted from the cell lines using an RNeasy mini kit (Qiagen, Valencia, USA) according to the standard protocol. RNA integrity was assessed using an Agilent 2100 Bioanalyzer (Agilent Technologies, Santa Clara, CA, USA). Total RNA $(1 \mu \mathrm{g})$ was reversetranscribed to complementary DNA (cDNA) using High-Capacity cDNA Reverse Transcription Kits (Applied Biosystems, Foster City, CA, USA). Expression of target genes was assessed using TaqMan qRT-PCR assays (Applied Biosystems). Relative gene expression was calculated by the $2^{-\Delta \mathrm{Ct}}$ method using GAPDH as the endogenous control for normalization.

To detect mature miR135b and miR642a expression levels, TaqMan quantitative real-time polymerase chain reaction (qRT-PCR) micro RNA (miRNA) assay (Applied Biosystems) was performed. The relative levels of expression of mature miR135b and miR642a normalized with respect to the RNU43 endogenous control were determined by the $2^{-\Delta \mathrm{Ct}}$ method. Each measurement was performed in triplicate.

\section{Transfections}

Cell lines were transfected using the nucleofector II system (Lonza, Allendale, NJ, USA) with the following 
programs: C-16 for H929 and JJN3, G-16 for MM1S, and X-005 for U266. Cells were transfected with onTARGET plus ${ }^{\mathrm{Tm}}$ control pool or on-TARGET plus SMART pool Human DEPTOR (Dharmacon, Lafayette, $\mathrm{CO}, \mathrm{USA}$ ); pre-miR ${ }^{\mathrm{Tm}}$ miRNA precursors pre-miR-135b, pre-miR-642a, and pre-miR ${ }^{\mathrm{Tw}}$ miRNA negative nontargeting control\#1 (Ambion, Austin, TX, USA); and microRNA inhibitors, hsa-miR-135b-5p miRCURY LNA $^{\text {ma }}$ microRNA inhibitor, hsa-miR-642a-5p miRCURY LNA $^{\text {ma }}$ microRNA inhibitor, and miRCURY LNA ${ }^{\mathrm{ms}}$ microRNA inhibitor negative control A (Exiqon, Woburn, MA, USA). Small interfering RNA (siRNA) and miRNA concentration of $25 \mathrm{nM}$ was used in all the experiments.

\section{Cell cycle analysis}

Cells were washed in PBS and fixed in 70\% ethanol for later use. Cells were rehydrated with PBS, resuspended in $500 \mu \mathrm{l}$ of PI/RNase staining solution (Immunostep), and incubated for $20 \mathrm{~min}$ at RT in the dark. Samples were analyzed using a FACSCalibur flow cytometer.

\section{Apoptosis and cell proliferation assays}

Apoptosis was measured using an annexin V-fluorescein isothiocyanate/propidium iodide (PI) double staining (Immunostep) according to the manufacturer's procedure. Cell viability was evaluated with the CellTiter-Glo ${ }^{\circ}$ luminescent cell viability assay based on the amount of ATP present (Promega), in accordance with the manufacturer's protocol.

\section{Immunophenotyping}

MM cell lines were immunophenotyped on a FACSCanto II cytometer (Beckton Dickinson Biosciences) using the following monoclonal antibodies: CD138OC515 (Cytognos S.L., Salamanca, Spain), CD38-APCH7 (BD Biosciences), and sIgk-PB (Vestec, Czech Republic). Data analysis was performed using the Infinicyt software (Cytognos S.L.). A minimum of $10^{5}$ events were stored. Median fluorescence intensity of each marker was analyzed.

\section{Luciferase reporter assay}

The double-stranded oligonucleotides corresponding to the wild-type or mutant miR135b and miR642a binding sites in the $3^{\prime}$-untranslated region (3'UTR) of DEPTOR were synthesized (Additional file 1: Table S1) (Sigma-Aldrich, St Louis, MO, USA) and ligated between the PmeI and $X b a l$ restriction sites of the pmirGLO vector (Promega, Madison, WI, USA). Oligonucleotide sequences are detailed in Additional file 1: Table S1. Luciferase assays in HEK293 cells were performed as previously described [18].

\section{Western blot}

Protein extraction and Western blot analysis were carried out as previously detailed [18]. The primary antibodies used for immunoblotting were anti-DEPTOR, anti-AKT, phospho-AKT (ser473), anti-p70 S6 kinase, anti-phosphop70 S6 (Thr389), anti-4E-BP1, anti-phospho-4E-BP1 (Thr37/46), anti-S6 ribosomal protein, anti-phospho-S6 ribosomal protein (Ser235/236) (Cell Signaling Technology, Beverly, MA, USA), anti-IRF4, anti-Ig kappa light chain, and anti-Ig lambda light chain (Santa Cruz Biotech, Delaware, CA, USA). Anti- $\beta$-actin (Sigma-Aldrich) was used as an internal control for protein loading. The membranes were then washed and incubated with the secondary horseradish per-oxidase-linked anti-mouse IgG or anti-rabbit IgG antibodies (PierceNet) (1:10000), anti-goat IgG (Santa Cruz Biotech) (1:10000). Chemiluminescence was detected using the Amersham ECL Plus ${ }^{\mathrm{Tm}}$ Western Blotting Detection Reagent (GE Healthcare).

\section{Capillary electrophoresis immunoassay}

Capillary electrophoresis immunoassay was performed using the $\mathrm{WES}^{\text {max }}$ machine (ProteinSimple Santa Clara, CA, USA) according to the manufacturer's protocol. In brief, $4 \mu \mathrm{l}$ of samples at a concentration of $0.1 \mathrm{mg} / \mathrm{ml}$ (or lower when it was not possible to achieve $0.1 \mathrm{mg} / \mathrm{ml}$ ) were combined with a master mix (ProteinSimple) to a final concentration of $1 \times$ sample buffer, $1 \times$ fluorescent molecular weight markers, and $40 \mathrm{mM}$ dithiothreitol (DTT), and then heated at $95{ }^{\circ} \mathrm{C}$ for $5 \mathrm{~min}$. The samples, blocking reagent, wash buffer, primary antibodies (anti-DEPTOR and anti-GAPDH at 1:100 concentration), secondary antibodies, and chemiluminescent substrate were dispensed into designated wells in the microplate provided by the manufacturer. After plate loading, the fully automated separation electrophoresis and immunodetection steps were carried out in the capillary system. Data were analyzed with the inbuilt Compass software (ProteinSimple). The signal from DEPTOR was normalized with respect to the signal from GAPDH, making sure that the signals of both proteins were within the linearity range.

\section{Immunofluorescence staining}

Cells were collected 48 or $72 \mathrm{~h}$ post-transfection, washed with PBS, and stained for $30 \mathrm{~min}$ with $1 \mu \mathrm{M}$ ER-Tracker $^{\text {rm }}$ Red (Invitrogen). Cells were washed again with PBS, fixed with $4 \%$ formaldehyde for $5 \mathrm{~min}$ at room temperature, placed on glass slides coated with poly-L-lysine, and stained for $1 \mathrm{~min}$ with DAPI II. The slides were then mounted using VECTASHIELD Mounting Medium (Vector Laboratories, Burlingame, CA, USA). Images were collected under a Zeiss confocal microscope equipped with 636/1.4 Oil Plan-APOCRHOMAT DIC. 


\section{Statistical analysis}

The two-tailed Student $t$ test or the two-tailed Welch $t$ test was used to analyze group differences in experiments when data showed equal or unequal variances, respectively. Data are reported as mean values \pm standard deviation (SD) of at least three determinations.

Progression-free survival (PFS) distribution curves were plotted using the Kaplan-Meier method; the logrank test was used to estimate the statistical significance of differences between the curves. The Cutoff Finder web application (http://molpath.charite.de/cutoff) was used to determine the optimal cutoff, defined as that yielding the most significant split discriminating shorter and longer survival, and identified by testing all possible cutoffs using the log-rank test [19]. Values of $p<0.05$ were considered statistically significant. All statistical analyses were conducted using the SPSS 21.0 program (IBM Corp. Released 2012. IBM SPSS Statistics for Windows, Version 21.0. Armonk, NY: IBM Corp).

\section{Results}

DEPTOR is overexpressed in plasma cells compared with B lymphocytes

In order to confirm the previously observed overexpression of DEPTOR by microarray analysis in NPC and myeloma cells relative to NBL (GSE6691 at GEO repository) [14] (Fig. 1a), we quantified DEPTOR mRNA levels by qRT-PCR in four BC populations. To this end, immature, naïve, memory $B$ cells and PCs were sorted from BM samples obtained from healthy donors. DEPTOR expression was found to be significantly higher in PCs than in all previous stages of differentiation (Fig. 1b), which suggested that this protein could be involved in PC maturation.

\section{DEPTOR knockdown induces dedifferentiation of myeloma cells}

To gain further insight into the potential role of DEPTOR in PC differentiation, we knocked down its expression in MMCLs by siRNA for $48 \mathrm{~h}$ and then assessed the expression of key genes involved in B cell maturation by qRT-PCR. Cell growth and apoptosis experiments revealed that DEPTOR knockdown did not alter cell viability (Additional file 1: Figure S1 a and b). However, we found that PAX5 and BCL6, which both encode $\mathrm{B}$ cell lineage-specific activator proteins and which are present only at early stages of B cell differentiation [9], were increased in DEPTOR-silenced cells (Fig. 2a). Conversely, IRF4, an essential transcription factor for PC differentiation [20], was downregulated after DEPTOR knockdown. Reduced IRF4 levels were also confirmed by Western blot, mainly in H929 cells (Fig. 2b). Additionally, immunophenotypic markers related to $B$ cell differentiation, such as CD19, CD38, CD138, and $\mathrm{k}$ light chain were also assessed. Thus, expression levels of CD38, CD138, and k light chain were found to be lower, while CD19 expression was higher after DEPTOR silencing (Fig. 2a-c). Next, we analyzed the effect of DEPTOR downregulation on myeloma cell morphology. Clear reductions in cell size and endoplasmic reticulum (ER) mass were found in both $\mathrm{H} 929$ and MM1S as a consequence of DEPTOR downregulation (Fig. 3a-c). To exclude the possibility that cell cycle profiles were responsible for the differences in size of myeloma cells, we determined the percentage of cells in G1, S, and G2/M by flow cytometry. No differences in cell cycle profiles were observed between control and DEPTOR-silenced cells (Fig. 3d). Next, we also determined whether the observed reduction in cell size in DEPTOR-silenced cells could also be detected by flow cytometry. A clear reduction in mean FSC value was observed in DEPTOR-silenced cells compared with control cells (Fig. 3e). Taken together, these results indicate that DEPTOR downregulation in MM cells induces a reversal of PCs to previous stages of PC differentiation.

\section{Dedifferentiation of myeloma cells induced by DEPTOR silencing is independent of mTOR signaling}

As DEPTOR has been described previously as an mTORC1/mTORC2 inhibitor [13], we were interested
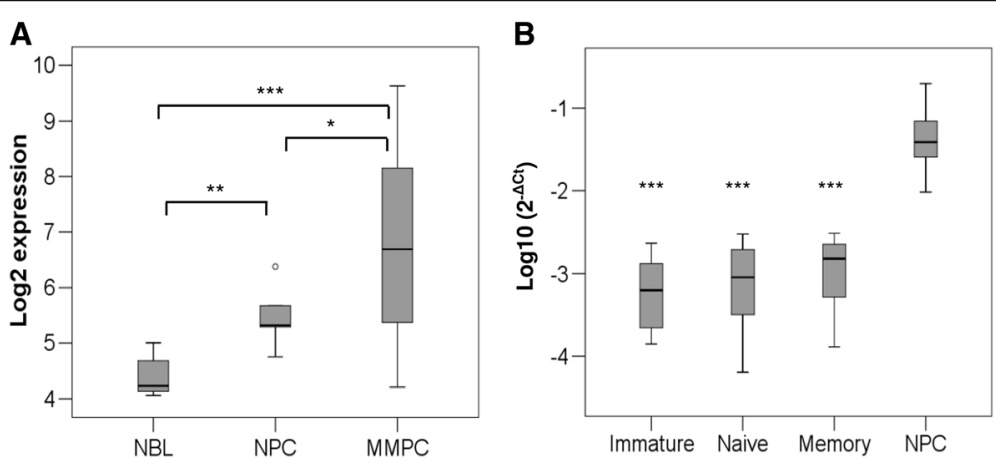

Fig. 1 DEPTOR expression. a DEPTOR expression in normal B lymphocytes (NBL), normal PC (NPC), and myeloma cells obtained from gene expression arrays (GSE6691 at GEO repository). b DEPTOR expression detected by qRT-PCR in immature B cells, naive B cells, memory B cells, and NPC. $\left({ }^{*} P<0.05\right.$, $\left.{ }^{* *} p^{<} 0.01,{ }^{* * *} p<0.001\right)$ 


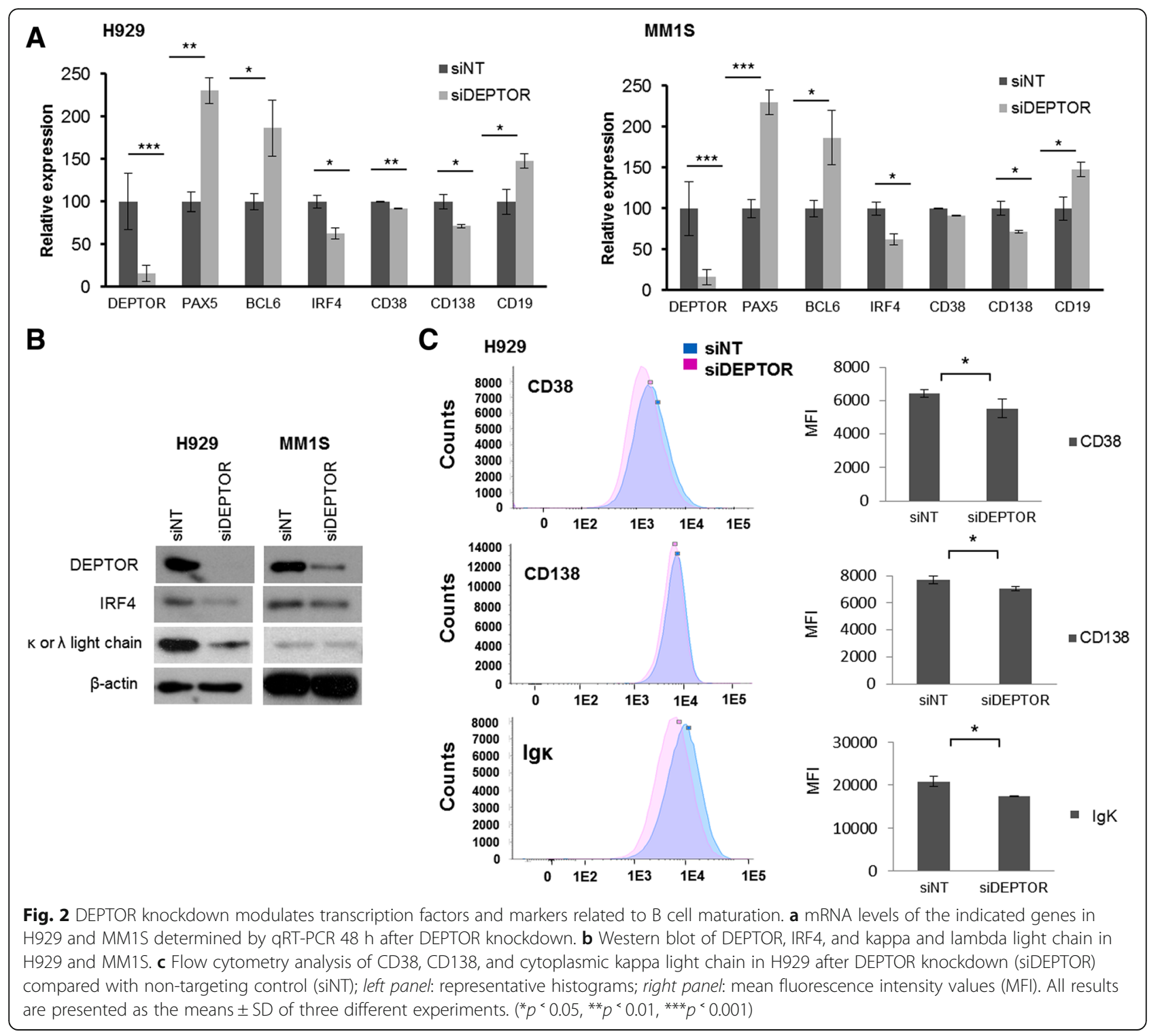

to determine whether PC dedifferentiation observed after DEPTOR silencing was induced through the mTOR signaling pathway. For this purpose, mTORC1 and mTORC2 activity was monitored through the phosphorylation state of their substrates using the same conditions of DEPTOR knockdown that induced PC dedifferentiation. We found that the levels of mTOR substrates were not changed by DEPTOR knockdown under the conditions assayed (Fig. 4a). These results implied that all the molecular and morphological changes obtained after DEPTOR knockdown were independent of the mTOR pathway. To confirm these findings, we added rapamycin, a well-known mTORC1 inhibitor, in the DEPTOR knockdown experiments. As shown in Fig. 4b, levels of p-S6 were again found to be similar in control and in DEPTOR knockdown cells, but lower in cells exposed to rapamycin, showing that the downregulation of IRF4 triggered by DEPTOR silencing was not reverted by the addition of rapamycin. Therefore, the PC state is maintained by DEPTOR independent of its role as an mTORC inhibitor.

DEPTOR is a direct target of miR135b and miR642a

It has previously been shown that DEPTOR is overexpressed in the subset of MM carrying MAF/MAFB and $C C N D 1 / C C N D 3$ translocations. The influence of MAF/ MAFB on DEPTOR levels was confirmed by both ectopic expression of MAFB and MAF silencing, which induced DEPTOR upregulation and downregulation, respectively [13]. We evaluated DEPTOR expression in a panel of MM cell lines with different chromosomal translocations (Fig. 5a). DEPTOR mRNA and protein 


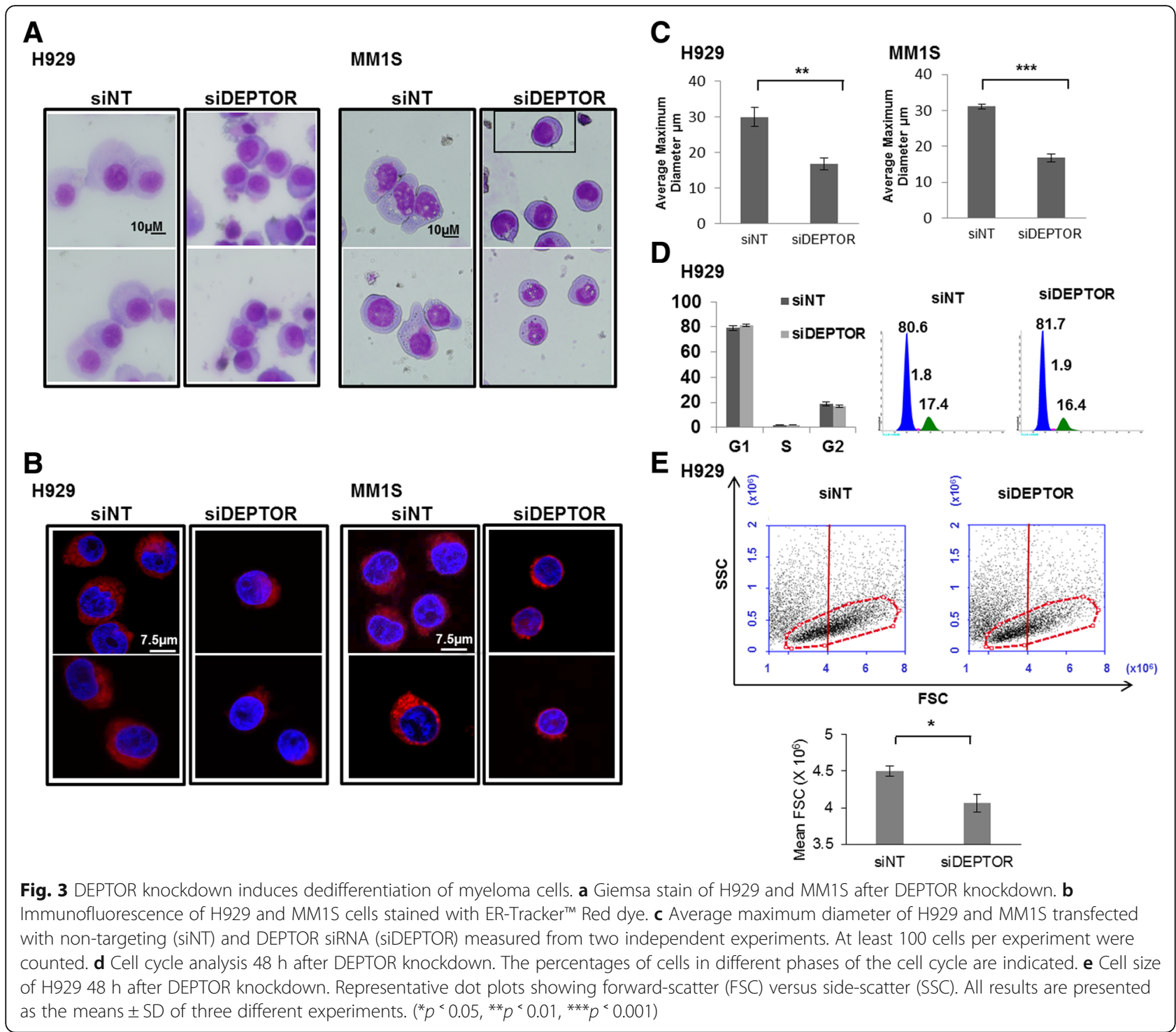

levels were detected in almost all cell lines analyzed, especially in RPMI-8226 and MM1S, which overexpress MAF [21], but also in H929 and OPM2 carrying the $(4 ; 14)$ translocation. Surprisingly, some MM cell lines exhibited low levels of DEPTOR, like JJN3, even though it carries a $M A F$ translocation, and U266, which overexpress cyclin D1 [22-26]. These results suggest that, although DEPTOR levels may be influenced by MAF/ MAFB or cyclin D1/3 expression, additional mechanisms may affect its expression. To address the possibility that post-transcriptional regulation by microRNAs is involved in DEPTOR expression, we looked for miRNADEPTOR interactions in five databases (miRMap, PITA, RNA22, RNAhybrid, and Targets scan), using a value of $p=0.05$ for miRNAs whose predicted binding site is the 3'UTR of DEPTOR (Additional file 1: Figure S2). A total of 47 common miRNAs were predicted to target
DEPTOR from the five combined datasets. Of these, $\mathrm{miR} 135 \mathrm{~b}$ and miR642a had been previously reported to be downregulated in $\mathrm{MM}$ with different cytogenetic abnormalities [27]. Underexpression of both miRNAs in different MM patients compared with NPCs was confirmed by qRT-PCR (Fig. 5b). According to the prediction algorithms, miR642a and miR135b have one putative site in the DEPTOR 3'UTR (Fig. 5c). To determine whether DEPTOR was a direct target of those miRNAs, we carried out luciferase reporter assays. The 3' UTR of DEPTOR harboring the sequence complementary to the miR642a or miR135b seed sequence was cloned in a reporter plasmid vector referred to as wildtype (WT). In parallel, a 3'UTR DEPTOR fragment containing mutant sequences (MUT) of the seed site of the two miRNAs was cloned in the same reporter plasmid. DEPTOR 3'UTR WT and MUT luciferase 


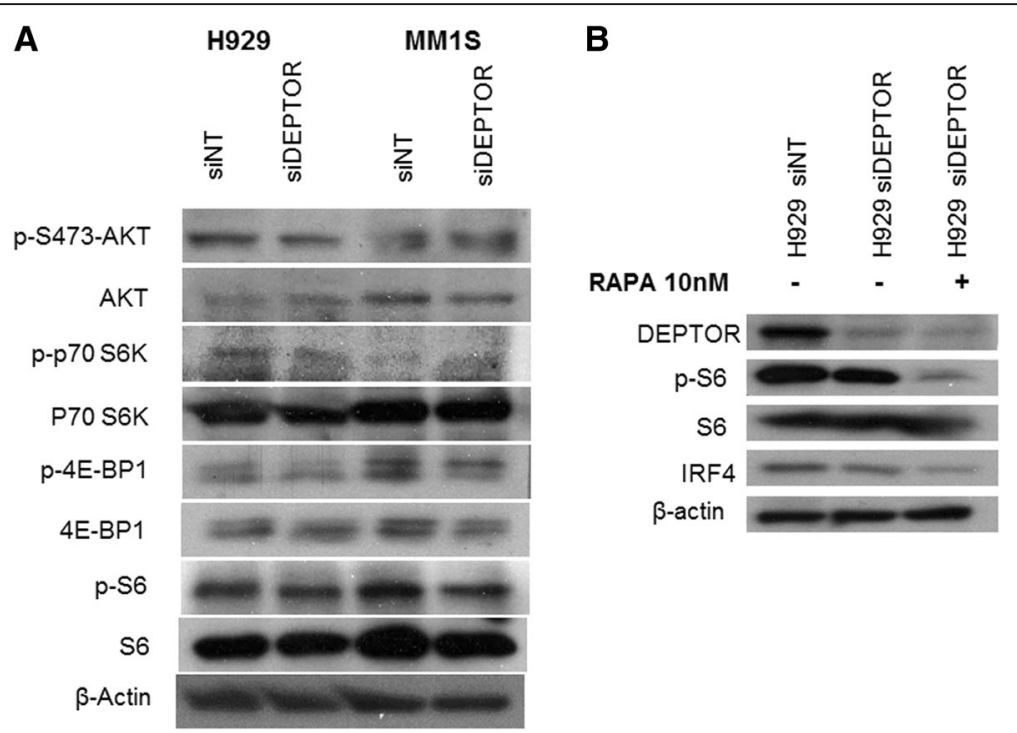

Fig. 4 Dedifferentiation of myeloma cells after DEPTOR silencing is independent of mTOR signaling. a Levels of mTORC1 and mTORC2 substrates after DEPTOR knockdown in H929 and MM1S. All experiments were carried out $48 \mathrm{~h}$ post-transfection. b Western blot of H929 transfected with siNT or siDEPTOR and cultured with control medium and rapamycin at $10 \mathrm{nM}$

constructs were then transfected into HEK293 cells along with $\mathrm{miR}-135 \mathrm{~b} / \mathrm{miR}-642 \mathrm{a}$ or negative control (NC) miRNA, and luciferase activity was determined. We found that luciferase activity of cells cotransfected with WT DEPTOR 3'UTR and miR135b or miR642a was significantly lower $(p<0.01)$ than that exhibited by cells transfected with NC control miRNA. Luciferase activity of MUT constructs was not affected by miR135b or miR642a overexpression (Fig. 5d).

\section{miR135b and miR642a regulate DEPTOR expression in MM}

Once $D E P T O R$ had been validated as being a direct target of miR642a and miR135b, we speculated that differences in DEPTOR levels among MM cell lines could be partly related with the endogenous levels of these miRNAs. In fact, using qRT-PCR, we found that JJN3 and U266, which exhibited low levels of DEPTOR despite MAF or cyclin D1 overexpression (Fig. 6a), expressed higher levels of miR642a and miR135b than H929, which displayed high levels of DEPTOR and carried $t(4 ; 14)$ (Fig. 6a). To test this hypothesis, H929 was transfected with miR135b or miR642a and NC miRNA precursors. Clearly, lower levels of DEPTOR protein expression were found in miR135b and miR642a transfected cells compared with control cells $72 \mathrm{~h}$ post-transfection (Fig. $6 \mathrm{~b}$ ). To gain further evidence of the relationship between miR642a/miR135b expression and DEPTOR levels, miR642a and miR135b were specifically knocked down in U266 and JJN3 cell lines. DEPTOR protein expression was significantly higher in both cell lines after transfection with miR642a/miR135b inhibitors (Fig. 6c). Taken together, these results demonstrate that miR135b and miR642a modulate DEPTOR expression through the consensus miR135b or miR642a-binding sites in DEPTOR 3'UTR. These miRNAs can subsequently participate in the regulation of DEPTOR expression in MM.

\section{Upregulation of miR135b and miR642a results in myeloma cell dedifferentiation through the negative regulation of DEPTOR}

Next, we were interested to determine whether downregulation of DEPTOR induced by the overexpression of miR135b or miR-642a also led to PC dedifferentiation. Consistent with the finding after DEPTOR silencing by siRNAs, the downregulation of DEPTOR by transfection of $\mathrm{H} 929$ cells with miR135b or miR642a resulted in downregulation of IRF4 and $k$ light chain proteins (Fig. 7a). In addition, DEPTOR knockdown by miR135b and miR642a overexpression led to the appearance of smaller, rounder cells with a less cytoplasm and ER content than control cells (Fig. 7b and c). These results and those obtained from siRNA experiments clearly indicated that the presence of DEPTOR is required to maintain myeloma cells at the terminal stage of differentiation.

\section{DEPTOR is differentially expressed in MM and its} upregulation is associated with longer survival and the stage of PC maturity

DEPTOR mRNA is known to be differentially expressed in $\mathrm{MM}$ patients $[13,28]$. We confirmed these results using two different microarray studies (GSE16558, and GSE39925 at GEO repository) [27, 29]. Next, we decided to quantify DEPTOR protein levels by capillary 
A

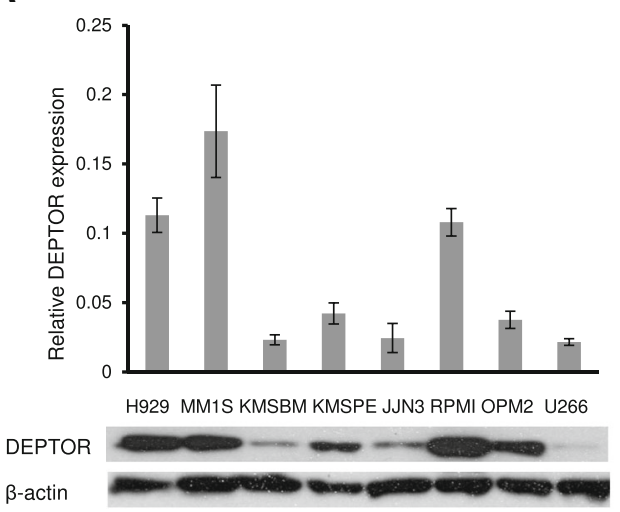

C

D

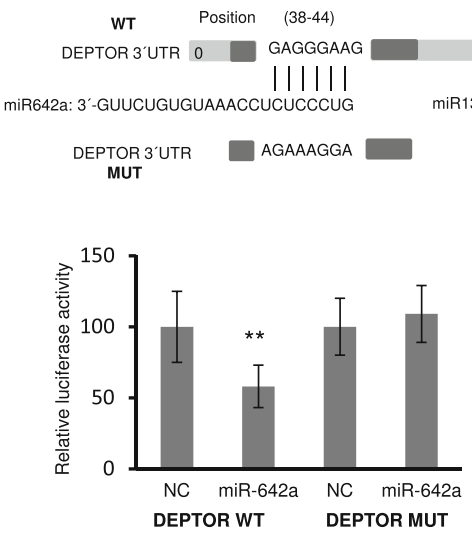

B
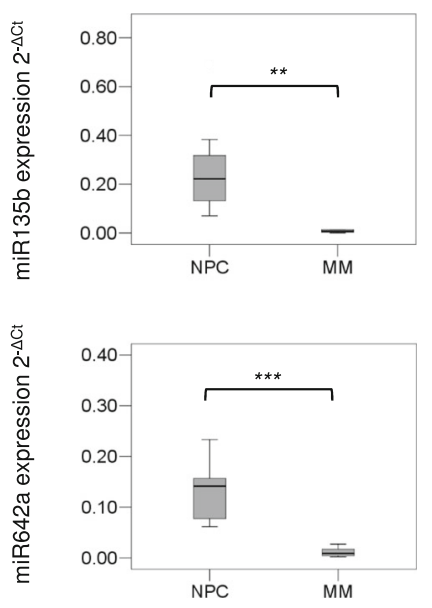

Position (530-537)

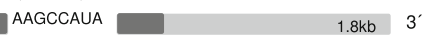

|l||||

AAGCTGCG

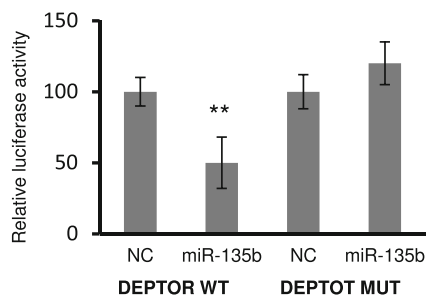

Fig. 5 DEPTOR expression is controlled by miR135b and miR642a in MM. a DEPTOR mRNA levels measured by qRT-PCR (upper panel). Levels of DEPTOR in MM cell lines detected by Western blot (lower panel). $\mathbf{b}$ miR135b and miR642a expression in NPC and MM patients measured by qRT-PCR. microRNA expression was normalized to RNU43 ( $\left.2^{-\Delta c t}\right)$. Two-tailed Welch's $t$ test $p$ values for miR135b and miR642a were calculated $(0.0081$ and 0.0001 , respectively). c Schematic diagram of the miR642a and miR135b predicted site on the DEPTOR 3'UTR, dark squares on DEPTOR 3'UTR WT, and DEPTOR 3'UTR MUT represents fragments cloned in pmiRGlo plasmid. $\mathbf{d}$ Luciferase activity in HEK293 cells cotransfected with pre-miR-NC or pre-miR135b/ miR642a and plasmid pmiR-Glo with the putative miR135b/miR642a binding site of DEPTOR cloned downstream of the luciferase reporter gene. Luciferase activity was normalized using Renilla. All results are presented as the means \pm SD of three different experiments. $\left({ }^{*} p^{<} 0.05,{ }^{* *} p^{<} 0.01,{ }^{* * *} p^{<} 0.001\right)$

electrophoresis immunoassay in myeloma cells from 24 MM patients treated according to the Spanish GEM2010 trial. Consistent with the mRNA expression data, we observed that levels of DEPTOR protein also differed among the myeloma samples (Fig. 8a). Interestingly, we found that the PFS was significantly longer in MM patients with high expression levels of DEPTOR than in those with low DEPTOR expression levels $(p=$ 0.038) (Fig. 8b).

The differences in DEPTOR protein expression among MM patients led us to hypothesize that DEPTOR levels might be associated with the maturation state of myeloma cells of each patient, in line with our results from MMCLs. As morphological characteristics of myeloma cells are correlated with maturation stage [30], we examined the morphology of myeloma cells isolated from three patients with high DEPTOR protein levels and three with low protein levels. We observed that those cells harboring high levels of DEPTOR had an eccentric nucleus and large and extended cytoplasm, and were bigger than those exhibiting low levels of the protein (Additional file 1: Figure S3). These results support those obtained from MMCLs and indicate that a suitable level of DEPTOR is necessary for PC maturation.

\section{Discussion}

In this study, we showed that DEPTOR, a protein overexpressed in MM [13], maintains PC differentiation. We also found that miR135b and miR642a, downregulated in the disease, modulate DEPTOR levels in MM cells.

Initial studies of PC maturation reported that the final step of PC differentiation was irreversible [9, 31-33]. However, recent studies have revealed that alterations in IRE1, XBP1, FOXP1, PAX5, or BCL6/MTA3 may reprogram PCs to previous stages of differentiation [34-37]. In keeping with these reports, we found that DEPTOR 


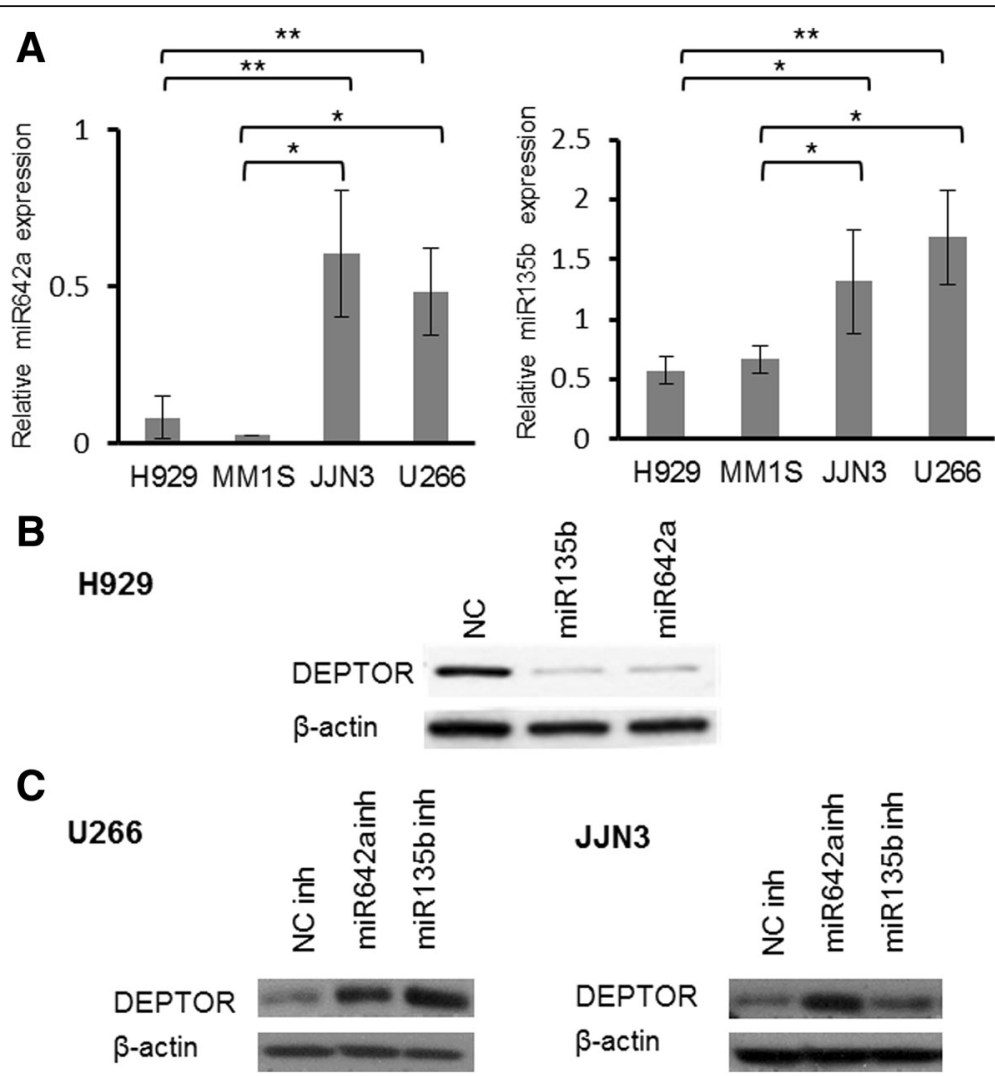

Fig. 6 miR642a and miR135b regulate DEPTOR expression in MM. a miR-135b and miR-642a expression in MM cell lines measured by qRT-PCR. microRNA expression was normalized with respect to RNU43 $\left(2^{-\Delta c t}\right)$. b Western blot of DEPTOR in H929 cells $72 \mathrm{~h}$ post-transfection with NC or miR-135b/miR-642a. c Western blot of DEPTOR in U266 and JJN3 transfected with NC or miR135b/miR642a inhibitors. ( ${ }^{*} p^{<} 0.05$, $\left.{ }^{* *} p<0.01,{ }^{* * *} p<0.001\right)$

knockdown changed the transcriptional program associated with PC differentiation through the upregulation of $P A X 5$ and BCL6, which maintain the B cell program, and downregulation of IRF4, a factor that favors PC differentiation [12]. The main function of $\mathrm{PC}$ is the production of immunoglobulins at high rate, and for this to happen, PCs must display a highly specialized morphology with expanded cytoplasm and a more sophisticated ER network compared with B cells [38]. Our results demonstrated a clear loss of cell size and ER mass in both H929 and MM1S as a consequence of DEPTOR downregulation. Taking these results together, we propose that DEPTOR maintains the state of PCs, and its deficiency in PCs results in PC dedifferentiation. Accordingly, DEPTOR levels increased during the differentiation of human PCs from B cells. Peterson et al.

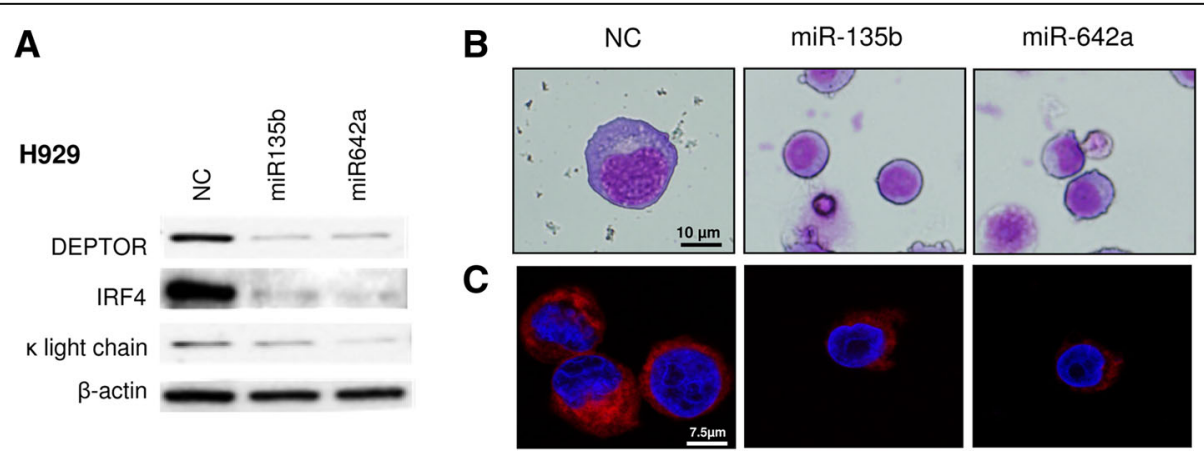

Fig. 7 Upregulation of miR135b and miR642a results in myeloma cell dedifferentiation through negative regulation of DEPTOR. a Western blot of DEPTOR, IRF4, and kappa light chain in $\mathrm{H} 929$ cells $72 \mathrm{~h}$ post-transfection with NC or miR-135b/miR-642a. b Giemsa stain of H929 cells transfected with NC or miR-135b/miR-642a. c Immunofluorescence of H929 cells stained with ER tracker 


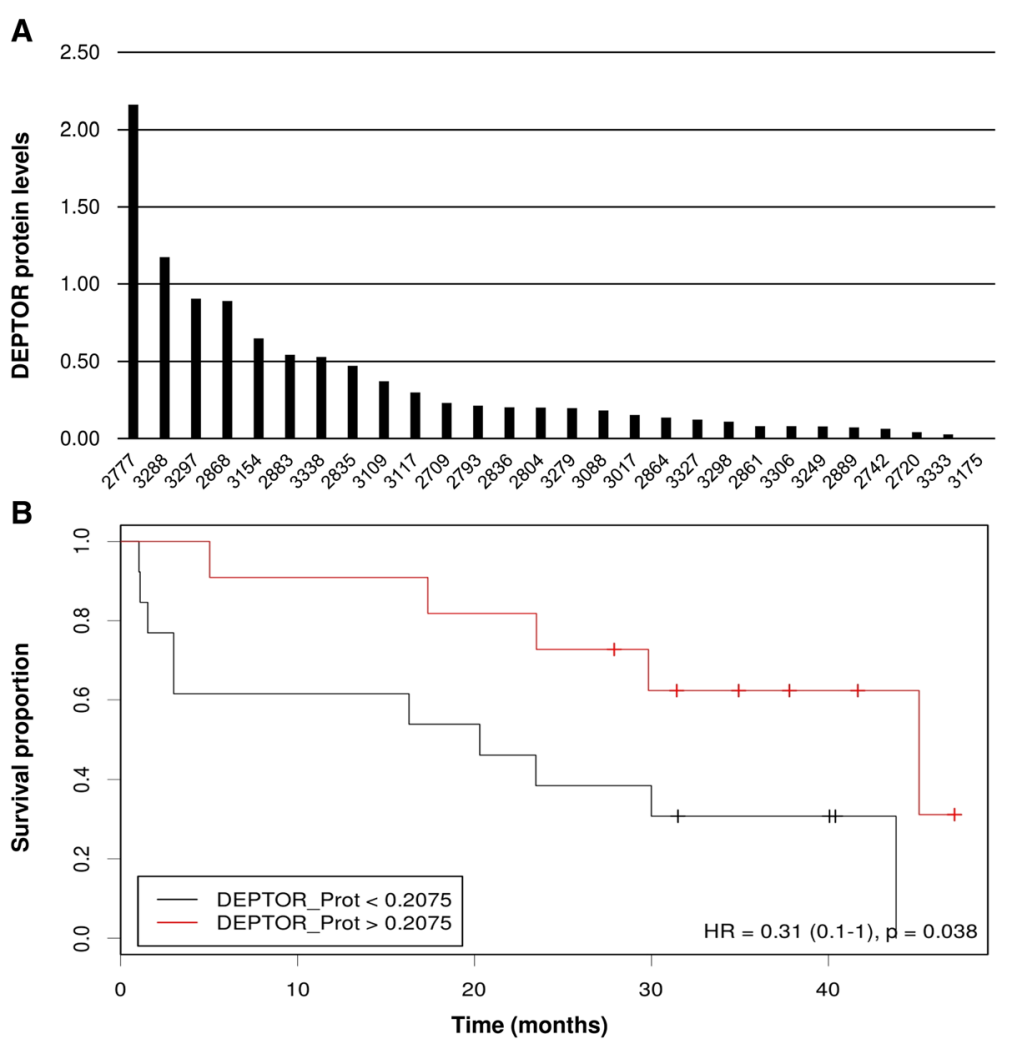

Fig. 8 DEPTOR is differentially expressed in MM and its overexpression is associated with longer survival. a Levels of DEPTOR in MM patients assessed using capillary electrophoresis immunoassay. b Kaplan-Meier curves for progression-free survival (PFS) in 24 MM patients treated according to GEM2010

showed that DEPTOR overexpression is necessary to maintain PI3K and AKT activation and that a reduction in DEPTOR levels leads to apoptosis [13].

Here, we found that DEPTOR inhibition, at the times and under the conditions assayed, did not affect cell survival, but reverted the commitment of PCs. A few earlier studies have found a connection between DEPTOR levels, mTORC1/mTORC2 activities, and cell differentiation. It has been reported that DEPTOR maintains stem cell pluripotency by limiting mTOR activity in undifferentiated embryonic stem cells (ESCs) [39], whereas differentiation of mouse ESCs is associated with decreased DEPTOR levels. In $T$ cells, it has been demonstrated that mTOR drives $\mathrm{T}$ cell differentiation and function $[40,41]$. In B cells, one study has analyzed the consequences of B cell-specific loss of the mTOR negative regulator TSC1 [42]. The authors showed that deletion of TSC1 in murine B cells and subsequent TORC1 activation led to impairment of $\mathrm{B}$ cell maturation. This work appears to be in agreement with our findings, in the sense that B cells would need an mTORC1 inhibitor to promote PC differentiation. However, we unexpectedly found that mTORC1/mTORC2 activities were not modified by DEPTOR silencing under our experimental conditions. The independence of mTORC1 activity was corroborated by the addition of rapamycin, an mTORC1 inhibitor that did not revert the PC dedifferentiation induced by DEPTOR knockdown. We hypothesize that DEPTOR may regulate $B$ cell differentiation through mTOR-independent pathways. Their molecular connections with PC differentiation need to be elucidated.

DEPTOR has been found to be overexpressed in many tumor types, including breast cancer, prostate cancer, chronic myeloid leukemia, lung cancer, and MM [16]. In the latter, DEPTOR overexpression was associated with cyclin D1/D3 upregulation and especially with the presence of $M A F / M A F B$ translocations [13]. The involvement of miRNAs in the pathogenesis and biology of myeloma has been suggested by several groups [27, 43, 44]. Here, we found that DEPTOR expression is also controlled by two miRNAs, miR135b and miR642a, both of which are downregulated in several MM patients [27]. Using luciferase reporter assays and gain-of-function experiments, we showed that transfection of miR135b and miR642a decreased DEPTOR levels in myeloma cells. Moreover, inhibition of miR135b and miR642a in two MMCLs exhibiting high levels of expression of both miRNAs and a low level of expression of DEPTOR, despite displaying MAF or cyclinD1 upregulation, resulted in DEPTOR overexpression. We observed that DEPTOR downregulation 
induced by miR135b and miR642a ectopic expression also reverted the transcriptional program of the myeloma cells and reduced cell size and ER mass, similarly to the results obtained from DEPTOR silencing by siRNA. These findings emphasize the role that these miRNAs play in regulating DEPTOR expression.

It was of particular note that DEPTOR protein levels in myeloma cells varied from patient to patient, and that its upregulation was clearly associated with longer PFS. Interestingly, high levels of DEPTOR expression have previously been associated with the prediction of response to thalidomide in MM [28]. This observation is consistent with the fact that the patients included in our study received a treatment regimen that contained lenalidomide, a thalidomide-like drug. On the other hand, we have shown that DEPTOR induces PC maturation, and it has been reported that the maturation of myeloma cells is associated with sensitivity to anti-myeloma agents [30, 34, 45-47], including lenalidomide [45]. In fact, plasma cell maturity seems to be an indicator of good prognosis in MM [47].

\section{Conclusions}

Overall, our results show that high levels of DEPTOR result in more mature myeloma cells that would be more sensitive to therapeutic agents. They suggest the merit of further investigations to test the potential of DEPTOR levels as an indicator of maturation and as a predictive biomarker of sensitivity to anti-myeloma therapy.

\section{Additional file}

Additional file 1: Table S1. List of oligonucleotide sequences used for $3^{\prime}$
UTR luciferase constructs. Figure S1. DEPTOR knockdown did not alter cell
viability. a Proliferation of myeloma cells 48 h after transfection with DEPTOR
siRNA. Data are expressed as means of three independent experiments \pm
SD. Proliferation of cells transfected with siNT was taken as 100\%, and values
obtained in DEPTOR-silenced cells were normalized accordingly. b Percent-
age of apoptosis after DEPTOR knockdown in H929 and MM1S. Right panel
shows representative dot plots. Figure S2. Bioinformatic identification of
miRNAs that regulate DEPTOR expression. Venn diagram showing numbers
of miRNAs predicted to target DEPTOR by the indicated five databases.
Figure S3. Cell morphology and size in MM patients with different DEPTOR
levels a Giemsa stain of three MM patients. b Average maximum diameter
of MM cells measured from patients harboring high ( $n=3$ ) and low $(n=3)$
DEPTOR levels. At least 50 cells per experiment were counted. ${ }^{*} p^{<} 0.05$,
${ }^{* *} p^{\left.<0.01,{ }^{* * *} p<0.001\right) .(D O C X ~} 1346$ kb)

\section{Abbreviations}

3'UTR: 3'-untraslated region; BCs: B cells; ER: Endoplasmic reticulum; miRNA: micro RNA; MM: Multiple myeloma; MMCL: Human multiple myeloma cell lines; MUT: Mutant sequence; NC: Negative control; NPCs: Normal PCs; PCs: Plasma cells; qRT-PCR: Quantitative real-time polymerase chain reaction; siRNA: Small interfering RNA; UPR: Unfolded protein response; WT: Wild-type sequence

\section{Acknowledgements}

The authors thank Isabel Isidro, Teresa Prieto, Vanesa Gutiérrez, and Irene Aires for their technical assistance. We would particularly like to acknowledge all the participating members of the Spanish Myeloma Group (PETHEMA/GEM).

\section{Funding}

This study was partially supported by the Instituto de Salud Carlos III (Fondo de Investigaciones Sanitarias: PI08/0568 and P113/00111), Asociación Española

Contra el Cáncer (AECC, GCB120981SAN), Gerencia Regional de Salud, Junta de Castilla y León grants (GRS 702/A11, BIO/SA57/13 and BIO/SA35/14), the Spanish Myeloma Network Program (RD12/0036/0058), and the INNOCAMPUS Program (CEI10-1-0010). IMK was supported by the Instituto de Salud Carlos III (PS09/01897) and the International Myeloma Foundation's Black Swan Research Initiative ${ }^{\circledast}$. MES was supported by Contrato Miguel Servet (CP13/00080).

\section{Availability of data and materials}

All the data generated or analyzed during this study are included in this published article [and its Additional file 1].

\section{Authors' contributions}

DQ designed the study, performed most of the experiments, and wrote the paper. LAC performed the bioinformatics and statistical analysis. IMK carried out the protein quantification in MM patients. MES and RGS carried out the sequencing of cloned fragment in pmiRGLO plasmid. JJP and NP carried out the immunophenotypic analysis and supervised the cell sorting experiments. MVM and RGS provided the patient samples. PK supervised the transfections and luciferase reporter assays. ABH and NCG designed the study, supervised the experiments, and corrected and approved the final version of the manuscript. All authors read and approved the final manuscript.

\section{Competing interests}

The authors declare that they have no competing interests.

\section{Consent for publication}

Not applicable.

\section{Ethics approval and consent to participate}

All patients and healthy donors provided written informed consent in accordance with the Helsinki Declaration. The research ethics committee of the University Hospital of Salamanca approved the study. Committee's name: Research ethics committee of the University Hospital of Salamanca, Spain. Approval file number CEIC 10/837. Date 26 April 2010.

\section{Publisher's Note}

Springer Nature remains neutral with regard to jurisdictional claims in published maps and institutional affiliations.

\section{Author details}

${ }^{1}$ Cancer Research Center-IBMCC (USAL-CSIC), Salamanca, Spain. ${ }^{2}$ Department of Hematology, University Hospital of Salamanca, Paseo San Vicente, 58-182, Salamanca 37007, Spain. ${ }^{3}$ Institute of Biomedical Research of Salamanca (IBSAL), Salamanca, Spain. ${ }^{4}$ National Medicines Institute, Warsaw, Poland.

Received: 19 January 2017 Accepted: 31 March 2017

Published online: 18 April 2017

\section{References}

1. Kyle RA, Rajkumar SV. Multiple myeloma. N Engl J Med. 2004;351:1860-73.

2. Somasundaram R, Prasad MA, Ungerback J, Sigvardsson M. Transcription factor networks in B-cell differentiation link development to acute lymphoid leukemia. Blood. 2015;126:144-52

3. Familiades J, Bousquet M, Lafage-Pochitaloff M, Béné M-C, Beldjord K, De Vos J, et al. PAX5 mutations occur frequently in adult B-cell progenitor acute lymphoblastic leukemia and PAX5 haploinsufficiency is associated with BCR-ABL1 and TCF3-PBX1 fusion genes: a GRAALL study. Leukemia. 2009;23:1989-98.

4. Traverse-Glehen A, Verney A, Baseggio L, Felman P, Callet-Bauchu E, Thieblemont C, et al. Analysis of BCL-6, CD95, PIM1, RHO/TTF and PAX5 mutations in splenic and nodal marginal zone B-cell lymphomas suggests a particular B-cell origin. Leukemia. 2007;21:1821-4. 
5. Kirk SJ, Cliff JM, Thomas JA, Ward TH. Biogenesis of secretory organelles during B cell differentiation. J Leukoc Biol. 2010;87:245-55.

6. King LB, Corley RB. Characterization of a presecretory phase in B-cell differentiation. Proc Natl Acad Sci U S A. 1989;86:2814-8.

7. Jourdan M, Caraux A, Caron G, Robert N, Fiol G, Reme T, et al. Characterization of a transitional preplasmablast population in the process of human B cell to plasma cell differentiation. J Immunol. 2011;187:3931-41.

8. Gass JN, Gunn KE, Sriburi R, Brewer JW. Stressed-out B cells? Plasma-cell differentiation and the unfolded protein response. Trends Immunol. 2004; 25:17-24.

9. Nutt SL, Taubenheim N, Hasbold J, Corcoran LM, Hodgkin PD. The genetic network controlling plasma cell differentiation. Semin Immunol. 2011;23 $341-9$.

10. Calame KL, Lin KI, Tunyaplin C. Regulatory mechanisms that determine the development and function of plasma cells. Annu Rev Immunol. 2003;21: 205-30.

11. Shapiro-Shelef M, Calame K. Regulation of plasma-cell development. Nat Rev. 2005;5:230-42.

12. Nutt SL, Hodgkin PD, Tarlinton DM, Corcoran LM. The generation of antibody-secreting plasma cells. Nat Rev. 2015;15:160-71.

13. Peterson TR, Laplante M, Thoreen CC, Sancak Y, Kang SA, Kuehl WM, et al. DEPTOR is an mTOR inhibitor frequently overexpressed in multiple myeloma cells and required for their survival. Cell. 2009;137:873-86.

14. Gutierrez NC, Ocio EM, de Las Rivas J, Maiso P, Delgado M, Ferminan E, et al. Gene expression profiling of B lymphocytes and plasma cells from Waldenstrom's macroglobulinemia: comparison with expression patterns of the same cell counterparts from chronic lymphocytic leukemia, multiple myeloma and normal individuals. Leukemia. 2007;21:541-9.

15. Catena V, Fanciulli M. Deptor: not only a mTOR inhibitor. J Exp Clin Cancer Res CR. 2017;36:12

16. Wang Z, Zhong J, Inuzuka H, Gao D, Shaik S, Sarkar FH, et al. An evolving role for DEPTOR in tumor development and progression. Neoplasia N Y N. 2012;14:368-75

17. Carrasco DR, Tonon G, Huang Y, Zhang Y, Sinha R, Feng B, et al. Highresolution genomic profiles define distinct clinico-pathogenetic subgroups of multiple myeloma patients. Cancer Cell. 2006;9:313-25.

18. Misiewicz-Krzeminska I, Sarasquete ME, Quwaider D, Krzeminski P, Ticona FV, Paino T, et al. Restoration of microRNA-214 expression reduces growth of myeloma cells through positive regulation of P53 and inhibition of DNA replication. Haematologica. 2013;98:640-8.

19. Budczies J, Klauschen F, Sinn BV, Gyorffy B, Schmitt WD, Darb-Esfahani S, et al. Cutoff Finder: a comprehensive and straightforward Web application enabling rapid biomarker cutoff optimization. PLoS One. 2012;7:e51862.

20. Klein U, Casola S, Cattoretti G, Shen Q, Lia M, Mo T, et al. Transcription factor IRF4 controls plasma cell differentiation and class-switch recombination. Nat Immunol. 2006;7:773-82.

21. Hurt EM, Wiestner A, Rosenwald A, Shaffer AL, Campo E, Grogan T, et al. Overexpression of $\mathrm{c}$-maf is a frequent oncogenic event in multiple myeloma that promotes proliferation and pathological interactions with bone marrow stroma. Cancer Cell. 2004;5:191-9.

22. Eychene A, Rocques N, Pouponnot C. A new MAFia in cancer. Nat Rev. 2008;8:683-93.

23. Gabrea A, Bergsagel PL, Chesi M, Shou Y, Kuehl WM. Insertion of excised IgH switch sequences causes overexpression of cyclin D1 in a myeloma tumor cell. Mol Cell. 1999:3:119-23.

24. Herath NI, Rocques N, Garancher A, Eychene A, Pouponnot C. GSK3mediated MAF phosphorylation in multiple myeloma as a potential therapeutic target. Blood Cancer J. 2014;4:e175.

25. Lombardi L, Poretti G, Mattioli M, Fabris S, Agnelli L, Bicciato S, et al. Molecular characterization of human multiple myeloma cell lines by integrative genomics: insights into the biology of the disease. Genes Chromosomes Cancer. 2007;46:226-38.

26. Chang $\mathrm{H}$, Qi Q, Xu W, Patterson B. c-Maf nuclear oncoprotein is frequently expressed in multiple myeloma. Leukemia. 2007;21:1572-4.

27. Gutierrez NC, Sarasquete ME, Misiewicz-Krzeminska I, Delgado M, Rivas JDL, Ticona FV, et al. Deregulation of microRNA expression in the different genetic subtypes of multiple myeloma and correlation with gene expression profiling. Leukemia. 2010;24:629-37.

28. Boyd KD, Walker BA, Wardell CP, Ross FM, Gregory WM, Davies FE, et al. High expression levels of the mammalian target of rapamycin inhibitor
DEPTOR are predictive of response to thalidomide in myeloma. Leuk Lymphoma. 2010;51:2126-9.

29. Todoerti K, Agnelli L, Fabris S, Lionetti M, Tuana G, Mosca L, et al. Transcriptional characterization of a prospective series of primary plasma cell leukemia revealed signatures associated with tumor progression and poorer outcome. Clin. Cancer Res. Off. J. Am. Assoc. Cancer Res. 2013;19: $3247-58$.

30. Goasguen JE, Zandecki M, Mathiot C, Scheiff JM, Bizet M, Ly-Sunnaram B, et al. Mature plasma cells as indicator of better prognosis in multiple myeloma. New methodology for the assessment of plasma cell morphology. Leuk Res. 1999;23:1133-40.

31. Shaffer AL, Lin Kl, Kuo TC, Yu X, Hurt EM, Rosenwald A, et al. Blimp-1 orchestrates plasma cell differentiation by extinguishing the mature $B$ cell gene expression program. Immunity. 2002;17:51-62.

32. Jr JEF. Self-perpetuating states in signal transduction: positive feedback, double-negative feedback and bistability. Curr Opin Cell Biol. 2002;14:140-8.

33. Monod J, Jacob F. Teleonomic mechanisms in cellular metabolism, growth, and differentiation. Cold Spring Harb Symp Quant Biol. 1961;26:389-401.

34. Leung-Hagesteijn C, Erdmann N, Cheung G, Keats JJ, Stewart AK, Reece DE, et al. Xbp1s-negative tumor B cells and pre-plasmablasts mediate therapeutic proteasome inhibitor resistance in multiple myeloma. Cancer Cell. 2013:24:289-304.

35. van Keimpema M, Gruneberg L, Mokry M, van Boxtel R, van Zelm MC, Coffer $\mathrm{P}$, et al. The forkhead transcription factor FOXP1 represses human plasma cell differentiation. Blood. 2015;126:2098-109.

36. Fujita N, Jaye DL, Geigerman C, Akyildiz A, Mooney MR, Boss JM, et al. MTA3 and the Mi-2/NuRD complex regulate cell fate during B lymphocyte differentiation. Cell. 2004:119:75-86.

37. Mikkola I, Heavey B, Horcher M, Busslinger M. Reversion of B cell commitment upon loss of Pax5 expression. Science. 2002;297:110-3.

38. Bettigole SE, Glimcher LH. Endoplasmic reticulum stress in immunity. Annu Rev Immunol. 2015;33:107-38.

39. Agrawal P, Reynolds J, Chew S, Lamba DA, Hughes RE. DEPTOR is a stemness factor that regulates pluripotency of embryonic stem cells. J Biol Chem. 2014;289:31818-26.

40. Powell JD, Delgoffe GM. The mammalian target of rapamycin: linking T cell differentiation, function, and metabolism. Immunity. 2010;33:301-11.

41. Chapman NM, Chi H. mTOR links environmental signals to T cell fate decisions. Front Immunol. 2015:5:686

42. Benhamron S, Tirosh B. Direct activation of mTOR in B lymphocytes confers impairment in B-cell maturation andloss of marginal zone B cells. Eur J Immunol. 2011:41:2390-6.

43. Pichiorri F, Suh SS, Ladetto M, Kuehl M, Palumbo T, Drandi D, et al. MicroRNAs regulate critical genes associated with multiple myeloma pathogenesis. Proc Natl Acad Sci U S A. 2008;105:12885-90.

44. Lionetti M, Biasiolo M, Agnelli L, Todoerti K, Mosca L, Fabris S, et al. Identification of microRNA expression patterns and definition of a microRNA/mRNA regulatory network in distinct molecular groups of multiple myeloma. Blood. 2009;114:e20-6.

45. Kawano Y, Fujiwara S, Wada N, Izaki M, Yuki H, Okuno Y, et al. Multiple myeloma cells expressing low levels of CD138 have an immature phenotype and reduced sensitivity to lenalidomide. Int J Oncol. 2012;41: 876-84.

46. Kuroda Y, Sakai A, Okikawa Y, Munemasa S, Katayama Y, Hyodo H, et al. The maturation of myeloma cells correlates with sensitivity to chemotherapeutic agents. Int J Hematol. 2005;81:335-41.

47. Iriyama N, Miura K, Hatta Y, Uchino Y, Kurita D, Takahashi H, et al. Plasma cell maturity as a predictor of prognosis in multiple myeloma. Med Oncol. 2016:33:87. 016-0803-3. Epub 2016 Jul 6. 